\title{
Understanding the Variable Drivers of Toxicity for the Broad Class of Carbon Nanotubes and Nanofibers from U.S. Facilities
}

\author{
Kelly E. Fraser \\ kzsmith@mix.wvu.edu
}

Follow this and additional works at: https://researchrepository.wvu.edu/etd

Part of the Other Pharmacy and Pharmaceutical Sciences Commons

\section{Recommended Citation}

Fraser, Kelly E., "Understanding the Variable Drivers of Toxicity for the Broad Class of Carbon Nanotubes and Nanofibers from U.S. Facilities" (2021). Graduate Theses, Dissertations, and Problem Reports. 8085. https://researchrepository.wvu.edu/etd/8085

This Dissertation is protected by copyright and/or related rights. It has been brought to you by the The Research Repository @ WVU with permission from the rights-holder(s). You are free to use this Dissertation in any way that is permitted by the copyright and related rights legislation that applies to your use. For other uses you must obtain permission from the rights-holder(s) directly, unless additional rights are indicated by a Creative Commons license in the record and/ or on the work itself. This Dissertation has been accepted for inclusion in WVU Graduate Theses, Dissertations, and Problem Reports collection by an authorized administrator of The Research Repository @ WVU.

For more information, please contact researchrepository@mail.wvu.edu. 


\title{
Understanding the Variable Drivers of Toxicity for the Broad Class of Carbon Nanotubes and Nanofibers from U.S. Facilities
}

\author{
Kelly E. Fraser \\ Dissertation submitted to the School of Pharmacy \\ at West Virginia University \\ in partial fulfillment of the requirements for the degree of \\ Doctor of Philosophy in \\ Pharmaceutical Sciences
}

\author{
Aaron Erdely, $\mathrm{PhD}$, Co-Chair \\ Vincent Castranova, $\mathrm{PhD}$, Co-Chair \\ Patrick Callery, $\mathrm{PhD}$ \\ Timothy Nurkiewicz, $\mathrm{PhD}$ \\ Yon Rojanasakul, $\mathrm{PhD}$
}

Department of Pharmaceutical Sciences

Morgantown, West Virginia, 2021

\footnotetext{
Keywords: Carbon nanotube, carbon nanofiber, nano- and advanced materials toxicology, physicochemical characterization, pulmonary toxicity, genotoxicity, inflammation, histopathology, extrapulmonary translocation, computer modeling
} 


\begin{abstract}
Understanding the Variable Drivers of Toxicity for the Broad Class of Carbon Nanotubes and Nanofibers from U.S. Facilities
\end{abstract}

Kelly E. Fraser

Pulmonary exposure to carbon nanotubes or nanofibers $(\mathrm{CNT} / \mathrm{F})$ is known to induce inflammation, toxicity, or tumorigenesis, and is a concern in the occupational setting. U. S. facility employees are at risk of inhalation exposure of multi-walled carbon nanotubes and carbon nanofibers during primary and secondary manufacturing. To date, only one MWCNT, Mitsui-7 has been classified as possibly carcinogenic to humans (Group 2B), while all other materials were subsequently categorized as unclassifiable (Group 3). This class of material has recently been listed as a high priority to the International Agency for Research on Cancer due to this significant knowledge gap. Furthermore, expressed desire to better understand the toxicity profiles of these materials has emerged from the National Institute for Occupational Safety and Health. While human research to date is limited, the use of in in vivo and in vitro model systems can be implemented for the assessment of toxicity outcomes following respiratory exposure to $\mathrm{CNT} / \mathrm{F}$. The goal of this study was to generate an accurate an effective safety profile of MWCNT and CNFs from U. S. facilities, and to adapt a multi-disciplinary approach using machine learning to identify pertinent physicochemical characteristics that act as drivers of these toxicity outcomes.

This study established toxicity profiles from male C57BL6/J mice aged 8-10 weeks exposed to either 4 or $40 \mu \mathrm{g}$ of one of nine different CNT/F via oropharyngeal aspiration as well as human epithelial BEAS-2B cells $(0-24 \mu \mathrm{g} / \mathrm{ml})$, differentiated THP-1 cells $(0-120 \mu \mathrm{g} / \mathrm{ml})$, and human fibroblasts $(0-2 \mu \mathrm{g} / \mathrm{ml})$ for four primary outcomes of genotoxicity, inflammation, pathology, and translocation. The nine materials used in this study had a wide range of characteristics including diameter (6-397 nm), length $(0.1-50 \mu \mathrm{m})$, surface area (18-238 $\left.\mathrm{m}^{2} / \mathrm{g}\right)$, aspect ratio (2-1396), residual metal catalyst (0.3-6.2 \%), density (0.007-0.220 $\mathrm{g} / \mathrm{cm}^{3}$ ), etc., to consider.

Toxicity profiles were generated regarding these four primary toxicity outcomes, and both supervised and unsupervised machine learning was used to identify the key drivers of these adverse health effects. While some physicochemical characteristics were determined to be key drivers of specific toxicity outcomes, different characteristics were essential when considering other toxicity endpoints. No single characteristic could be used as a toxicity predictor, therefore, multifactorial processes, or combination of characteristics, were necessary for an accurate and effective prediction model for responses. The study identified physicochemical drivers of $\mathrm{CNT} / \mathrm{F}$ toxicity using an integrated approach, combining experimental evidence with computational modeling, with potential for broad application. This study provides necessary information for the consideration of the potential human health effects that can result from CNT/F exposure. The safety profiles and identified drivers of toxicity may be useful for future predictive risk assessment studies and translational studies as well as contributing to safety-by-design for future material designs. 


\section{ACKNOWLEDGMENTS}

First, I would like the thanks my mentor and advisor, Dr. Aaron Erdely. Despite my lack of background in anything regarding the lungs, nanoscience, and particle toxicity, you accepted me into your laboratory and immediately began training me to be the scientist I am today. My previous experiences only highlighted how incredible of a mentor you have been, by not just teaching me how to conduct an experiment, but how to manage enormous studies, effectively communicate, manage a huge network of collaborators, and succeed within a professional work environment. Your teaching has been above and beyond what I expected from my graduate training, and I'm incredibly thankful for all your time, effort, support, sarcasm, and guidance. My success in this program would not have happened if I had not taken that leap and joined your lab. Thank you.

I would also like to thank the other members of the lab for all your help and support. Tracy Eye, you have been a fantastic teacher of not just science and laboratory techniques, but in how to be a better scientist, student, friend, and mother. To Dr. Vamsi Kodali, Lindsay Bishop, and John Hubczak, thank you for all your support, contributions, and guidance. All of you made this lab into a wonderful place to work, so thank you.

There are so many other people at NIOSH that have made an impact on my growth as a graduate student. Dr. Robert Mercer, thank you for the countless hours reviewing my tedious excel formulas, and for teaching me such specialized skills in morphometry. Your insights into my project have been appreciated and helped to elevate my research to a whole other level. The Dr. Naveena Yanamala, thank you for all your expertise and time. This project has become something amazing thanks to your insights and efforts. To Dr. Patti Erdely and Dr. Mohammed Shoeb, thank you for including me in your research studies. You have helped me to expand my knowledge and skills beyond the small world of carbon nanotubes. I cannot even begin to list the countless others who have provided me with feedback and assistance on everything from posters to journal clubs. Thank you.

To Dr. Ava Winn, Lori Battelli, Rebecca Salmen, Mary Ann Hammer, Sherri Friend, Diana Richardson, Brenda Billig, Lindsay Grosse, and so many others, thank you for all your contributions to my project, from slicing and staining tissue, to the hours spent at the microscope, to the emotional support over cups of coffee at the end of the hallway. Special thanks as well to Taylor Trainor. You have been such an incredible friend and I am so thankful for all your support and laughs.

Thank you to all my collaborators and co-authors. All 35 of you. I am so thankful for all your help, guidance, and contributions to the project. This project brought together the expertise from engineering, computer science, epidemiology, and life sciences to become an immense, multi-disciplinary project and I am immeasurably grateful.

I would like to give a special thank you to my committee members, Dr. Vincent Castranova, Dr. Patrick Callery, Dr. Timothy Nurkiewicz, and Dr. Yon Rojanasakul, and to faculty and staff at WVU including Dr. Grazyna Szklarz, Dr. Werner Geldenhuys, Dr. Kimberly Quedado, and the entire Office of Graduate Research and Education for all your support though these years in my studies and research. I have grown so much as a researcher and student thanks to your guidance.

To all of my family and friends- thank you. This journey as a graduate student has been longer than I expected, but I'm thankful for every minute of it. My mom, Joyce Smith, dad, Vince Smith, and sister, Jenny Smith, have been an amazing support team. Thanks for listening to me and supporting me! To Bob Fraser, Danna Friend, and the whole Fraser and Friend families- thank you for becoming my family. To all other friends and family, thank you for everything you have done to support and encourage me in the tough times and to celebrate me in my accomplishments.

I would like to thank my husband, Justin Fraser for everything that you have done. You have been my rock through all of this, the good, the bad, the stress, and the success. I love you more than the mostest and cannot wait for what our future brings us. Thank you for being my other half and for being smart in all the ways I'm not. I owe a special thanks to Elsie, my daughter. Starting from feeling your first kicks to hearing your first giggles has been a great motivator, and I love you dearly. I hope your first words aren't “carbon nanotubes.” 


\section{TABLE OF CONTENTS}

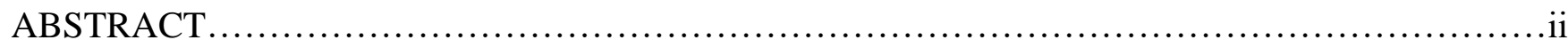

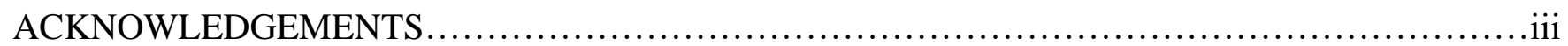

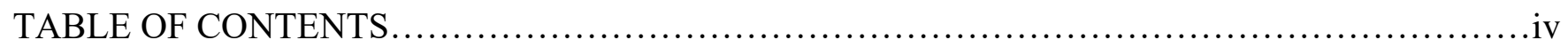

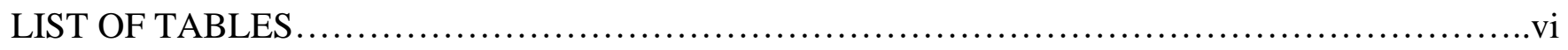

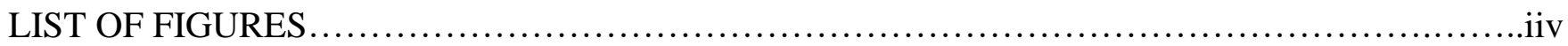

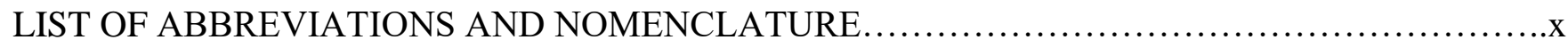

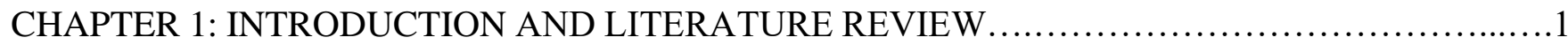

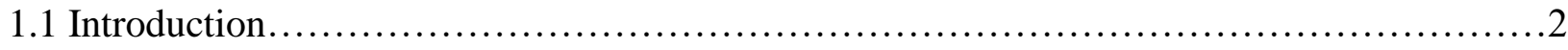

1.2 Key Physicochemical Characteristics..................................................... 3

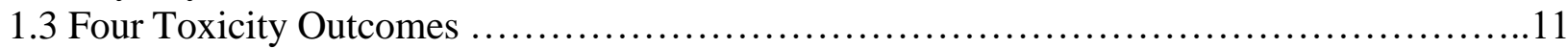

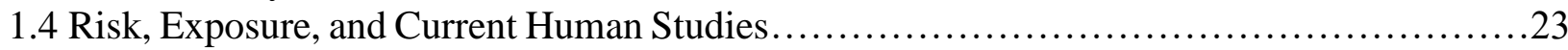

1.5 Conclusions and Justification of the Current Study ....................................

CHAPTER 2: PHYSICOCHEMICAL CHARACTERIZATION AND GENOTOXICITY OF THE BROAD CLASS OF CARBON NANOTUBES AND NANOFIBERS USED OR PRODUCED IN U.S. FACILITIES ...................................33

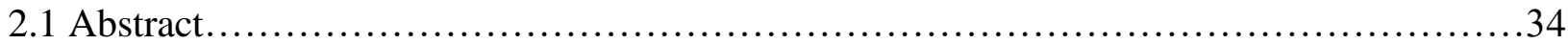

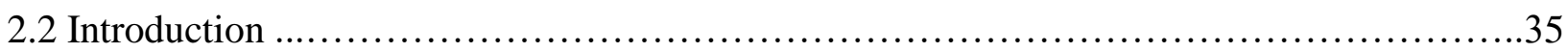

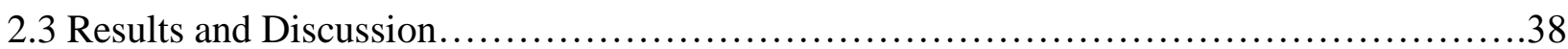

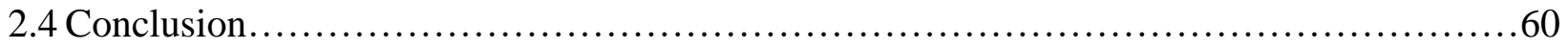

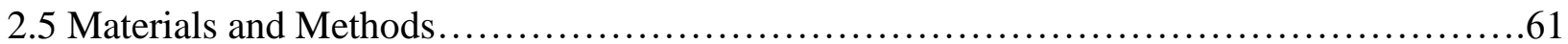

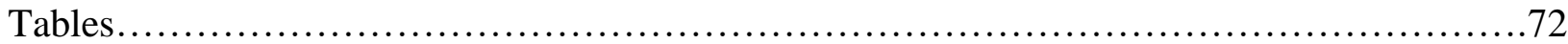

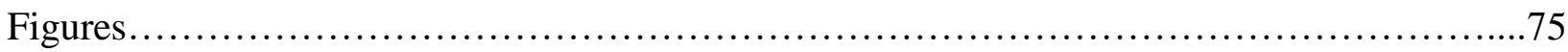

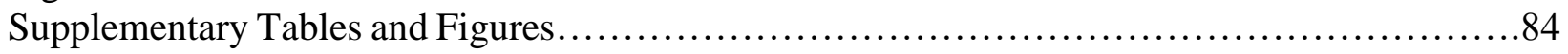

CHAPTER 3: INFLAMMATION OF THE BROAD CLASS OF CARBON NANOTUBES AND NANOFIBERS USED OR PRODUCED

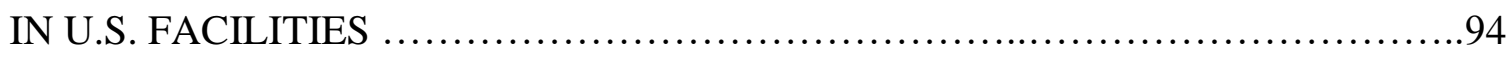

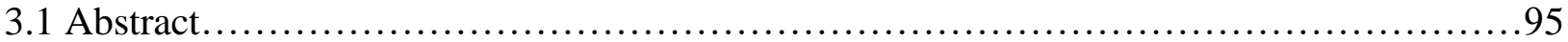

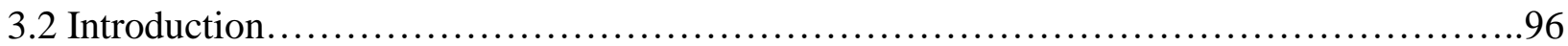

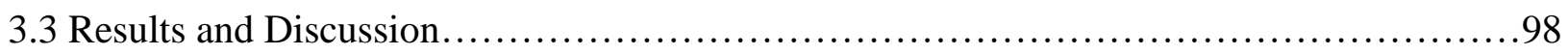

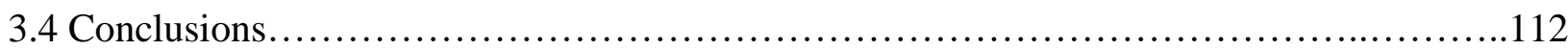

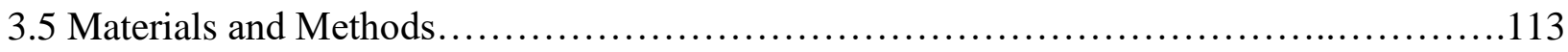

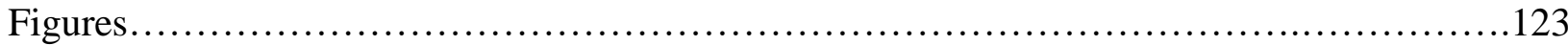

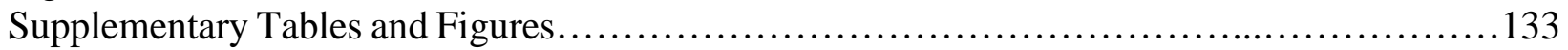




\section{CHAPTER 4: HISTOPATHOLOGY AND TRANSLOCATION OF THE}

BROAD CLASS OF CARBON NANOTUBES AND NANOFIBERS USED OR PRODUCED IN U.S. FACILITIES.

4.1 Abstract.

4.2 Introduction 151

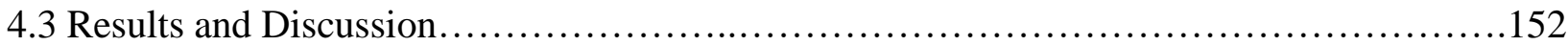

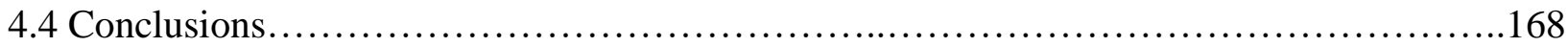

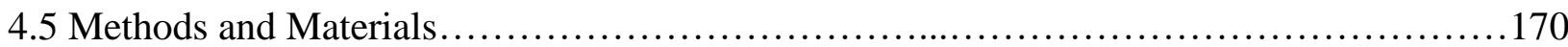

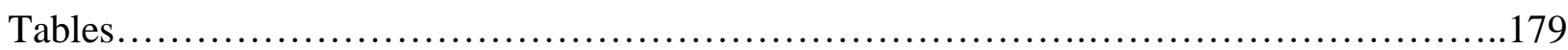

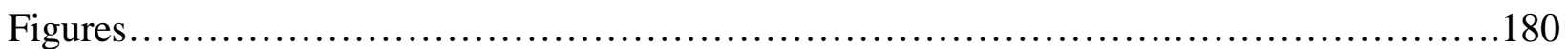

Supplementary Tables and Figures....................................................

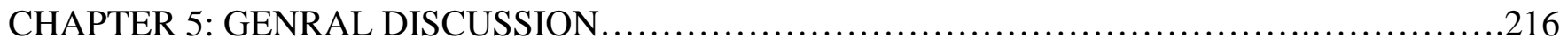

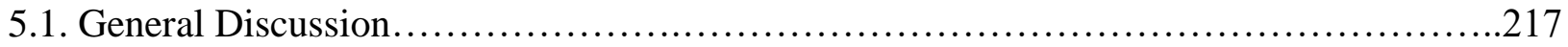

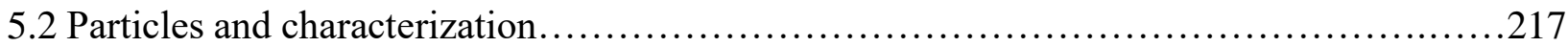

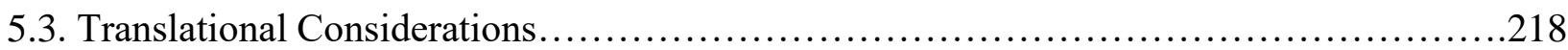

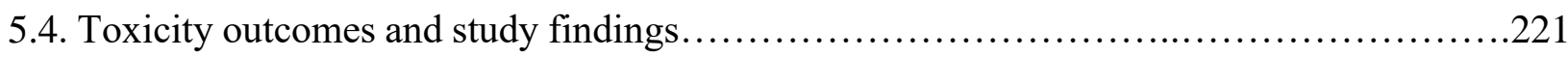

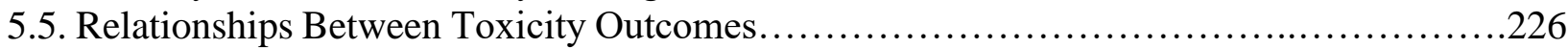

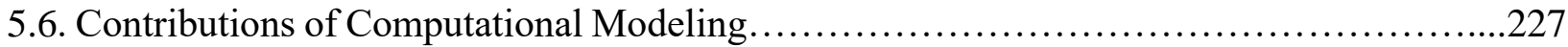

5.7. Impact and Significance on Safety Recommendations...............................228

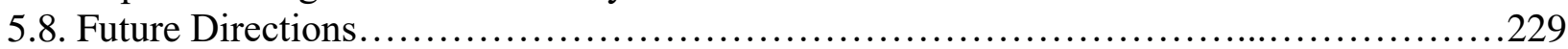

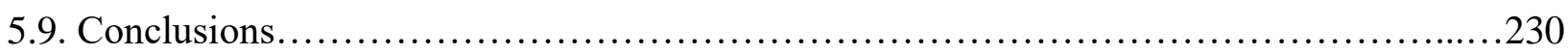

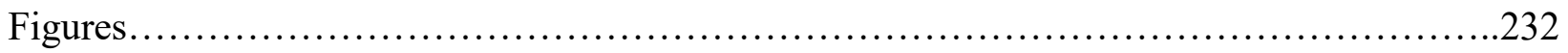

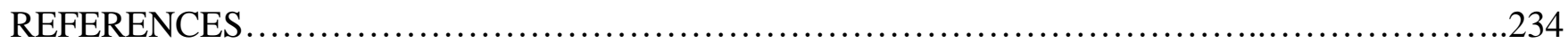




\section{LIST OF TABLES}

\section{Chapter 2:}

Table 1: Physical dimensions of CNT/F dispersed in isopropanol

Table 2: Hydrodynamic diameter, zeta potential, and two-dimensional sizing of CNT/F agglomerates dispersed in dosing media

Table 3: Additional particle characterization of CNT/F

Supplemental Table 1: Supernatant protein concentrations following CNT/F exposure in BEAS-2B cells

\section{Chapter 3:}

Supplemental Table 1: Physical dimensions of CNT/F dispersed in isopropanol

Supplemental Table 2: Hydrodynamic diameter, zeta potential, and two-dimensional sizing of $\mathrm{CNT} / \mathrm{F}$ agglomerates dispersed in dosing media

Supplemental Table 3: Additional particle characterization of CNT/F

Supplemental Table 4: Inflammatory cell counts of total cells and cell differentials listed as means and standard errors of raw values

Supplemental Table 5: Changes in mRNA expression of inflammatory cytokines and chemoattractants listed as means and standard errors

\section{Chapter 4:}

Table 1: Histopathology severity, distribution, and incidence scoring in the lung at 84 days post high dose exposure

Supplemental Table 1: Physical dimensions of CNT/F dispersed in isopropanol

Supplemental Table 2: Hydrodynamic diameter, zeta potential, and two-dimensional sizing of $\mathrm{CNT} / \mathrm{F}$ agglomerates dispersed in dosing media

Supplemental Table 3: Additional particle characterization of CNT/F 


\section{LIST OF FIGURES}

\section{Chapter 2:}

Figure 1: Schematic of material diameter and TEM images of CNT/F

Figure 2: Representative scanning electron microscopy images of CNT/F in dispersion media to measure two-dimensional agglomerate sizes

Figure 3: Distributions of CNT/F diameter

Figure 4: Distributions of CNT/F length

Figure 5: Distributions of CNT/F aspect ratio

Figure 6: Principal component analysis of different CNT/F materials comparing 'all characterization' parameters, 'length-width-aspect ratio (L-W-AR) physical dimensions, and 'means only' physicochemical characteristics

Figure 7: Toxicity assessment of BEAS-2B cells exposed to CNT/F

Figure 8: Genotoxicity assessments

Figure 9: Clustering of physicochemical characteristics with the epithelial cell toxicity outcomes of cell viability, cellular oxidative stress, $\gamma \mathrm{H} 2 \mathrm{AX}$, and micronuclei formation

Supplementary Figure 1: Variable importance plots for all characterization, L-W-AR binning, and means only

Supplementary Figure 2: Principle component analysis plots without feature selection plots for all characterization, L-W-AR binning, and means only

Supplementary Figure 3: Principle component analysis results from all characterization, L-WAR binning, and means only, combined with the four primary in vitro assay outcomes

Supplementary Figure 4: Hierarchical cluster analysis for all characteristics and the four primary in vitro outcomes along with inflammatory protein production

Supplementary Figure 5: Hierarchical cluster analysis without aspect ratio binning for all characterization, L-W-AR binning, and means only when considering the four primary in vitro epithelial toxicity outcomes

Supplementary Figure 6: Hierarchical cluster analysis without aspect ratio binning for all characterization, L-W-AR binning, and means only when considering the four primary in vitro epithelial toxicity outcomes and protein changes

Supplementary Figure 7: Hierarchical cluster analysis and principle component analysis without aspect ratio binning for the four primary in vitro epithelial toxicity outcomes and protein production only

\section{Chapter 3:}

Figure 1: Representative electron microscopy images of macrophages isolated from bronchoalveolar lavage fluid at 7 days post-exposure

Figure 2: Inflammation outcomes at 7 days post exposure

Figure 3: Inflammation outcomes at 28 days post exposure

Figure 4: Inflammation outcomes at 84 days post exposure

Figure 5: Hierarchical cluster analysis (HCA) and principal component analysis (PCA) grouping of inflammation outcomes alone

Figure 6: Hierarchical cluster analysis (HCA) and principal component analysis (PCA) grouping of inflammation outcomes and physicochemical characterization using 'all characterization' parameters 
Figure 7: Hierarchical cluster analysis (HCA) and principal component analysis (PCA) grouping of inflammation outcomes and physicochemical characterization using length-width parameters.

Figure 8: Toxicity assessment of inflammation in THP-1 macrophage cells exposed to CNT/F

Figure 9: Inflammasome activation and toxicity assessment of THP-1 macrophage cells exposed to $\mathrm{CNT} / \mathrm{F}$

Figure 10: Hierarchical cluster analysis (HCA) and principal component analysis (PCA) grouping THP-1 inflammation outcomes alone and THP-1 inflammation outcomes with length- width (L-W) physicochemical characteristics

Supplementary Figure 1: Distributions of CNT/F diameter

Supplementary Figure 2: Distributions of CNT/F length

Supplementary Figure 3: Representative micrographs of CNT/F collected from personal breathing zone samples (PBZ) compared to particle dispersion in dosing media (DM)

Supplementary Figure 4: Schematic of the mouse dosing and euthanasia schedule

Supplementary Figure 5: Representative micrographs using standard light microscopy of inflammatory cells isolated from bronchoalveolar lavage fluid at 7 days post-exposure

Supplementary Figure 6: Lactate dehydrogenase and inflammatory cell infiltration at 1- day postexposure to $\mathrm{CNT} / \mathrm{F}$

Supplementary Figure 7: Changes in mRNA expression of inflammatory cytokines and markers of oxidative stress from lung tissue at all four time points expressed as a heat map

Supplementary Figure 8: Hierarchical clustering analysis comparing inflammatory outcomes from bronchoalveolar lavage fluid at all four times points

Supplementary Figure 9: Hierarchical clustering analysis (HCA) and principal component analysis (PCA) comparing inflammatory outcomes from bronchoalveolar lavage fluid with length-width and 'means only' parameters of physicochemical characterization

Supplementary Figure 9: Hierarchical clustering analysis (HCA) and principal component analysis (PCA) comparing proteins from bronchoalveolar lavage fluid with length-width and 'means only' parameters of physicochemical characterization

\section{Chapter 4:}

Figure 1: Representative TEM images of particle dispersed in isopropanol

Figure 2: Representative micrographs at 84 days post-exposure of pathologies typically seen following MWCNT and CNF exposure

Figure 3: Bronchiolar Fibrosis at 84 days post-exposure to $40 \mu \mathrm{g}$ dose of MWCNT or CNF

Figure 4: Alveolar Fibrillary Collagen quantification

Figure 5: Hierarchical clustering analysis (HCA) and principal component analysis (PCA) of different CNT/F materials comparing MWNCT/CNF and histopathology outcomes

Figure 6: Hierarchical clustering analysis (HCA) and principal component analysis (PCA) of different CNT/F materials and bronchi/bronchiolar histopathology outcomes and alveolar histopathology outcomes

Figure 7: Hierarchical clustering analysis (HCA) and principal component analysis (PCA) of different CNT/F materials comparing length - diameter physical dimension (L-W binning) combined with all histopathology outcomes

Figure 8: Hierarchical clustering analysis (HCA) and principal component analysis (PCA) of different CNT/F materials comparing bronchial/bronchiolar histopathology outcomes and alveolar pathology outcomes 
Figure 9: Human fibroblast in vitro cell viability, collagen-1 production, asmooth muscle actin production, and TGF $\beta$ secretion following exposure to MWCNT/CNF

Figure 10: Hierarchical clustering analysis (HCA) and principal component analysis (PCA) of different $\mathrm{CNT} / \mathrm{F}$ materials comparing in vitro fibroblast outcomes

Figure 11: Representative Dark Field Micrographs of lungs at 1 Day post-exposure to $40 \mu \mathrm{g}$ MWCNT or CNF

Figure 12: Lung singlet burden at 1 day post exposure predicts extra-pulmonary translocation at 84 days post-exposure

Figure 13: Representative Micrographs of Tracheobronchial Lymph Nodes at 84 Days postexposure to $40 \mu \mathrm{g}$ MWCNT or CNF

Figure 14: Representative Dark Field Micrographs of Tracheobronchial Lymph Nodes at 84 Days post-exposure to $40 \mu \mathrm{g}$ MWCNT or CNF

Figure 15: Hierarchical clustering analysis (HCA) principal component analysis (PCA) of different CNT/F materials comparing translocation outcomes alone, as well as length diameter physical dimension (L-W binning) with extra-pulmonary translocation outcomes.

Supplementary Figure 1: Distributions of CNT/F diameter

Supplementary Figure 2: Distributions of CNT/F length

Supplementary Figure 3: Bronchial/bronchiolar fibrosis as a percentage of the area of fibrosis over the total area of the field of view for least affected and most affected bronchoalveolar junctions

Supplementary Figure 4: Hierarchical clustering analysis (HCA) and principal component analysis (PCA) of 'All characterization' and all histopathology outcomes

Supplementary Figure 5: Hierarchical clustering analysis (HCA) and principal component analysis (PCA) of 'All characterization' and all bronchi-bronchiolar outcomes

Supplementary Figure 6: Hierarchical clustering analysis (HCA) and principal component analysis (PCA) of 'All characterization' and alveolar outcomes

Supplementary Figure 7: Hierarchical clustering analysis (HCA) and principal component analysis (PCA) of 'means only' and all histopathology outcomes

Supplementary Figure 8: Hierarchical clustering analysis (HCA) and principal component analysis (PCA) of 'means only' and bronchi/bronchiolar outcomes

Supplementary Figure 9: Hierarchical clustering analysis (HCA) and principal component analysis (PCA) of 'means only' and alveolar outcomes

Supplementary Figure 10: Hierarchical clustering analysis (HCA) and principal component analysis (PCA) of 'all characterization' and in vitro fibroblast outcomes

Supplementary Figure 11: Hierarchical clustering analysis (HCA) and principal component analysis (PCA) of 'means only' and (A) length-diameter binning (B) with in vitro fibroblast outcomes

Supplementary Figure 12: Previously published image of extra pulmonary translocation and particle accumulation of MW \#3 in the spleen

\section{Chapter 5:}

Figure 1: Schematic of the physicochemical characteristics, the four major toxicity outcomes, and the relationships between them 


\section{LIST OF ABBREVIATIONS AND NOMENCLATURE}

BAL- bronchoalveolar lavage

BALF- bronchoalveolar lavage fluid

BEAS-2B- human bronchial epithelial cells

BET- Brunauer Emmet Teller

CCL2- Chemokine (C-C motif) ligand 2

CCL6- Chemokine (C-C motif) ligand 6

CCL22- Chemokine (C-C motif) ligand 22

CIB- current intelligence bulletin

$\mathrm{CNF}$ - carbon nanofibers

CNT- carbon nanotubes

CRP- C-reactive protein

CXCL2- Chemokine (C-X-C motif) ligand 2

DLS- dynamic light scattering

DMEM- Dulbecco's modified Eagle medium

DNA- deoxyribonucleic acid

DM- dispersion media

EMT- epithelial-mesenchymal transition

EPT- extra-pulmonary translocation

FENO- fractional exhaled nitric oxide

FE-SEM- field emission scanning electron microscopy

FRAS- ferric reducing ability of serum

GC-MS SIM- gas chromatography-mass spectrometry with selected ion monitoring

GCP-2- Granulocyte chemotactic protein 2

G-CSF- Granulocyte-colony stimulating factor

GFP- green fluorescent protein

GM- geometric mean

GM-CSF- Granulocyte-macrophage colony-stimulating factor

GSD- geometric standard deviation

HARN- high aspect ratio nanoparticle

HBS- human blood serum

HCA- hierarchical clustering analysis

HMOX-1- Heme oxygenase 1

IARC- International Agency for Research on Cancer

ICP-AES- inductively couple plasma atomic emission spectroscopy

IFN $\beta$ - Interferon beta

IFN $\gamma$ - Interferon gamma

IL-1 $\alpha$ - Interleukin 1 alpha

IL-1 $\beta$ - Interleukin 1 beta

IL-1ra- Interleukin 1 receptor antagonist

IL-2- Interleukin 2

IL-4- Interleukin 4

IL-5- Interleukin 5

IL-6- Interleukin 6

IL-7- Interleukin 7

IL-8- Interleukin 8

IL-9- Interleukin 9 


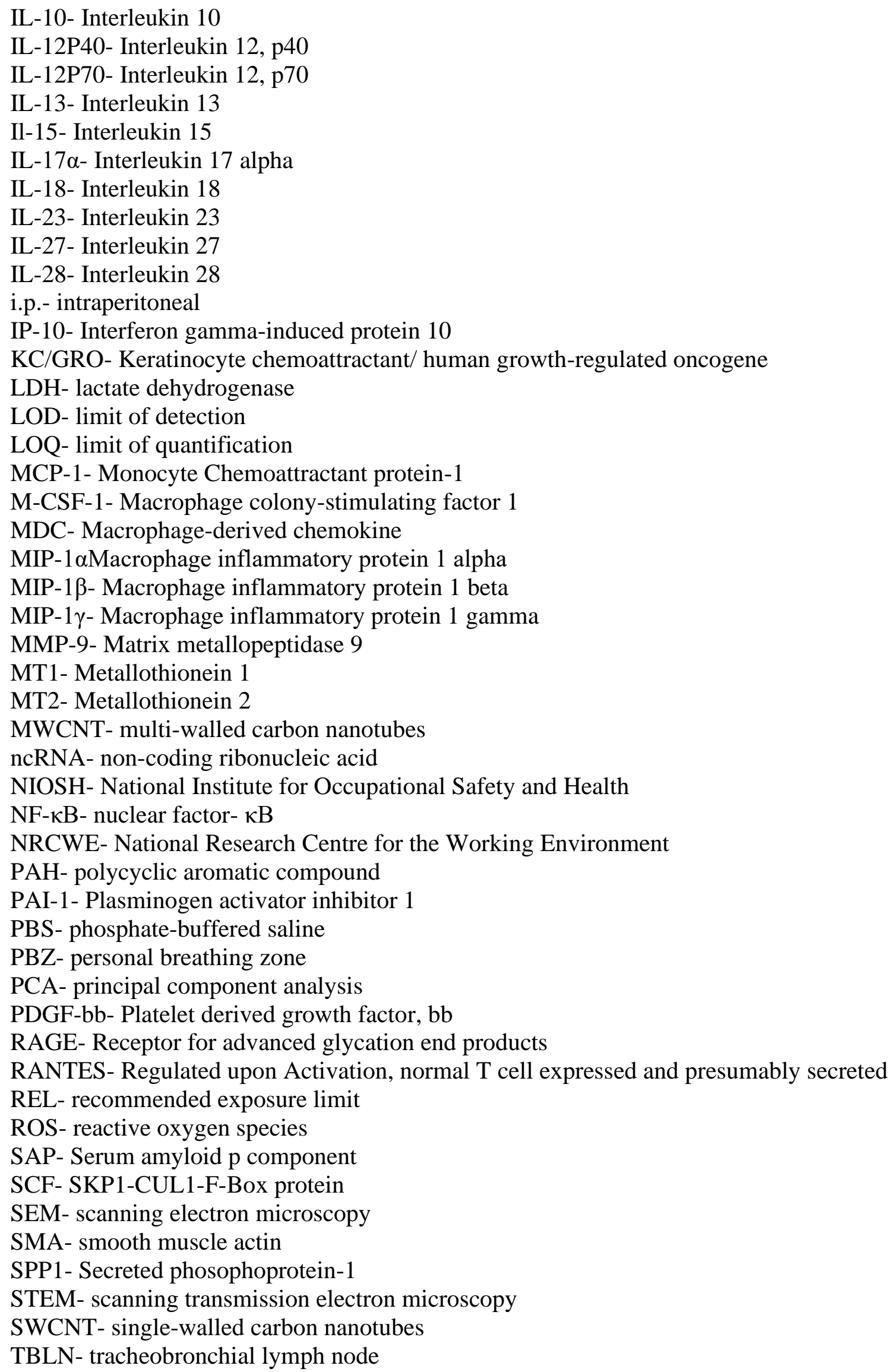


TGA- thermogravimetric analysis

THP-1- human macrophage cells

TIMP-1- TIMP Metallopeptidase inhibitor 1

TNF $\alpha$ - Tumor necrosis factor alpha

TPO- Thyroid peroxidase precursor

TSLP- Thymic stromal lymphopoietin

VCAM-1- Vascular cell adhesion molecule 1

VEGF-A- Vascular endothelial growth factor 1

WST-1- water soluble tetrazolium salts-1 


\section{CHAPTER 1:}

Introduction and Literature Review 


\subsection{Introduction}

The unique physicochemical properties of carbon nanotubes and nanofibers (CNT/F) make them a diverse class of immensely useful engineered nanomaterials with a multitude of applications. These applications are consistently growing resulting in the development of new materials, each with specialized properties suited for their intended use. Many properties of CNT/F are consistent and conserved between classes. For example, CNT/Fs have high tensile strength that is comparable to stainless steel yet have only a third of the density [1]. These properties, combined with being thermally and electrically conductive, make them ideal for use in composites, electronics, energy storage, and medical applications such as lenses, batteries, biosensors, and drug delivery systems. Of note, MWCNT and CNF are primarily used in composites. The addition of MWCNT and CNF to polymers, resins, and construction materials can generate composites that are stronger and more resilient without adding excess weight, allowing for applications in automotive, aerospace, and construction designs.

Beyond these universal properties, CNT/F can also be altered to have unique physicochemical properties to serve specialized functions. For example, CNT/F s can be coated or functionalized to alter surface chemistry resulting in differing dispersions, reactivity, durability, or application and uses [2-4]. However, these unique properties that make CNT/F s into such valuable materials may also be the same properties that drive their toxicities and adverse health outcomes following human exposure, primarily dermal and pulmonary exposures.

In the 1970s, Mearl F. Stanton led his research team and inspired subsequent studies to generate the fiber hypothesis. In his own words, "Precise parameters need not be defined to appreciate that carcinogenicity can depend on the structure rather than the physicochemical composition of a particle," [5]. This hypothesis stated that a fiber's toxicity is dependent on its physical characteristics of size, shape, dimension, and biopersistence. While these studies primarily focused on asbestos and glass fibers, this 
hypothesis can be expanded to all fibrous nanomaterials including CNTs, CNFs, nanowires, nanoribbons, and similarly high aspect ratio nanoparticles (HARNs).

\subsection{Key Physicochemical Characteristics}

Several key physicochemical characteristics must be well understood to address questions regarding CNT/F toxicities including length, diameter, agglomeration state, dustiness, metal content, and functionalization. These factors are all considered to be potential drivers of toxicities and will be explored further in the following sections.

\subsubsection{Length}

Historically, length has been a key characteristic in nanoparticle toxicities. Dating back through asbestos literature as well as nanowires, metallic nanofibers, and other HARNs, length has often been associated as being a key driving factor in nanofiber toxicity. These same assumptions have been applied to CNT toxicities. Numerous studies have linked CNT/F length to an increase in adverse health outcomes. Dating back to early 2010s, Donaldson and his collaborators published a series of publications investigating the link between particle length and toxicities in the lung and pleura of rodents. When directly exposed to peritoneal mesothelium, longer fibers, compared to shorter, tangled fibers, were more likely to induce greater inflammation and fibrosis in the pleural viscera, similar to previous asbestos findings [6, 7]. Like Stanton, this research suggested that increased particle length can result in more severe outcomes, likely due to diminished clearance capabilities. CNTs and CNFs can often be too large for macrophage uptake resulting in incomplete or "frustrated" phagocytosis. This frustrated phagocytosis acts as a driver for chronic inflammation and subsequent progressive pathologies and are often of note in determining the carcinogenicity of the material [8]. Donaldson et al. investigated pathological changes in the pleural viscera while groups such as Mercer et al. investigated changes in the lung tissue, also in rodent 
models $[6,9,10]$. Both concluded that diminished particle uptake can be linked to the development of fibrosis as well as lead to the formation of granulomas. Overall, particle length is an important characteristic responsible for the progression of adverse outcomes in the lung and can be linked to carcinogenicity, inflammation, and histopathology changes.

\subsubsection{Diameter}

While length is often considered to be a greater consideration regarding $\mathrm{CNT} / \mathrm{F}$ aspect ratio, diameter is still a critical factor. Thinner materials, and therefore greater aspect ratio, must be specially considered under the fiber hypothesis. In comparison to length, the role of diameter as a driver of toxicity outcomes in previously published literature has been less understood. In a series of publications from the National Research Centre for the Working Environment (NRCWE) (Denmark), carbon nanotubes of greater diameter were less likely to induce inflammation, genotoxicity, and histopathology changes including fibrosis and the granulomatous response [11, 12]. Similarly, when comparing single-walled carbon nanotubes (SWCNT) to MWCNTs, Mercer et al, found that SWCNTs were more fibrotic than MWCNTs, though this difference was linked to potential changes in macrophage uptake and particle accumulation in the alveolar interstitium [9, 10]. Macrophages had a greater capability to clear MWCNTs, therefore reducing interstitial accumulation and associated fibrosis. Furthermore, in a paper published in 2012 by Fenoglio et al. investigated two CNTs of different diameters, 9.4 and $70 \mathrm{~nm}$, and found that thinner particles induced greater lactase dehydrogenase release, a classic marker of cell damage, increased reactive oxygen species (ROS) production, linked to oxidative stress, and greater inflammatory cell infiltration to the lung [13]. However, the in vivo study findings were reported 24 hours post-exposure by aspiration to a high dose of $2 \mathrm{mg}$, which may have induced artificial bolus responses. In contrast to these findings, Nagai et al. (2011) completed a study in which rats were exposed via intraperitoneal (i.p.) 
injection. Thinner, less rigid particles were more likely to induce a greater frequency and malignancy of mesothelioma, as well as a lower survival rate than thicker CNTs [14].

These studies indicate that the diameter may play a role in determining toxicity outcomes, but a lack of cohesive study design as well as the range of size differences included in the study can lead to conflicting or unclear conclusions. A comparative study inclusive of a wide representation of particle sizes as well as ensuring translational relevance is necessary to fully understand the role of diameter and its influence on toxicity outcomes.

\subsubsection{Agglomeration}

Agglomeration of a particle can be a critical determinant in the type of response induced within exposed tissue. CNT/Fs can form agglomerates depending on their rigidity, size, production process, and surface chemistry. For assay purposes, both in vivo and in vitro, the preparation of the particle for dosing can also impact particle agglomeration as a secondary, artificial effect [15].

To simplify agglomeration, two general types of agglomerates can be formed, though these agglomerates can vary widely in shape and size. First, are spherical agglomerates. These agglomerates are often referred to as "cotton ball-like," "bird's nest," or "cooked spaghetti" in shape and their extent of entanglement [13]. As these particles are tightly tangled together into small clusters of particle, they are more difficult to disperse and can be as large as several microns in diameter. The second type of agglomerates are referred to as "bundles," "rope-like," or "rod-like" and can also contain tangles containing as few as just a couple of tubes together, or several together, often in parallel with one another. These agglomerates have a distinctly measurable length and diameter, compared to spherical agglomerates that generally only have a diameter. These can range anywhere from less than a dimeter in length, to tens of microns in length. 
Agglomeration is of special concern when considering pulmonary deposition. Su and Cheng completed a study using a human nasal replica and both SWCNTs and CNFs (stacked cups CNT) [16]. In this study, the aerodynamic diameter of the particle was quantified, and the respiratory deposition of the particle was assessed. All particles in this study were able to reach beyond the nasal passages and deposit in at least the bronchioles of the lung, though it was indicated to be more likely that deposition of the particles could reach the alveolar regions of the lung. Other studies have indicated that CNTs can be deposited in the alveolar space and can be found within the alveolar interstitium [9]. Some studies have attempted computer modeling of agglomerate sizes as a means of predicting regions of pulmonary deposition related to agglomerate size and shape $[17,18]$. Other experimental studies have also shown evidence that agglomerate size can alter the region of deposition within the lung as well as how those aggregates can accumulate and "stack" with one another to form aggregates of deposited particle. In an earlier study in 2017, particle deposition related to agglomerate size had been investigated and evidence suggested that particles of larger agglomerate size may be more likely to be deposited in the bronchi and bronchioles prior to the broncho-alveolar duct, limiting tissue injury to airways excluding the alveolar region [19]. For this reason, it is necessary to develop methodology to properly quantify particle agglomeration as a means to predict regional pulmonary deposition and the associated toxicity outcomes.

\subsubsection{Dustiness}

Dustiness is a measurement that confers the likelihood of aerosolization of a particle, or the ability to become airborne, and the subsequent ability for that particle, when inhaled, to bypass the nasal passages and reach the deeper airways of the lung. Two values can be quantified per the methodology established by Evans et al. in 2013 [20]. First, the total dustiness is fraction of the particle that can be aerosolized and is often considered the inhalable fraction, while the respirable fraction is the portion of the total dustiness 
capable of reaching the alveolar region of the lung. Factors such as density, particle size, dimensions, and agglomeration are all factors that contribute to particle dustiness.

Dustiness is an essential consideration when determining the likelihood of particle exposure. Particles with decreased dustiness, and therefore less propensity for aerosolization, have a reduced possibility of inhalation. Overall, this can reduce the likelihood of human exposures by reducing the quantity of inhaled and respired particle both by number of particles and the mass of total particle exposure. Furthermore, dustiness, when considered in pair with aerodynamic diameter, may be important in determining the biokinetics of particle respiration.

Previous studies have attempted to investigate the relationship between particle dustiness and toxicity outcomes. In the previous study by Bishop et al. (2017), two particles, both as produced and coated with a proprietary coating (four total particles), were characterized and extensively investigated for toxicity outcomes including genotoxicity, inflammation, and histopathology [19]. In one instance, the coating decreased the dustiness by an order of magnitude, while the second company's coated particle had an increase in dustiness, also by an order of magnitude. While the coating of these particle may have altered some outcomes such as histopathology changes, these changes were more likely to be a result of changes in recognition by immune cells including macrophages. The region of deposition was not significantly different regardless of dustiness, and histopathology outcomes were suspected to be due to changes in clearance, independently of dustiness. Therefore, dustiness may not alter outcomes once exposure has occurred, but reduction of the overall likelihood of exposure can still be beneficial to reducing human adverse outcomes.

\subsubsection{Metal Content}

Several methods of CNT/F production exist, including laser ablation, arc discharge, and chemical vapor deposition. While these methods remain useful, chemical vapor deposition methods for CNT/F 
production are most applicable for large scale, or large quantities of particle production. This method for production allows for several adaptable parameters during production stages to alter the physicochemical properties of CNT/F end products, including nominal diameter, nominal length, and morphology. Altering the catalyst chemicals present, the size and shape of the catalyst, reaction times, and temperature can all contribute to variations in generated CNT/F end products [21]. Metals are the most commonly used catalyst in these reactions, and their use is referred to as metal loading [21]. The most commonly used metals include cobalt, iron, nickel, and molybdenum, though metals such as aluminum, cadmium, magnesium, manganese, and more can be employed [15, 22-24]. Due to the nature of CNT/F growth during production, these metals often remain in the final product in detectable quantities through methods including inductively coupled plasma atomic emission spectroscopy (ICP-AES) and thermogravimetric analysis (TGA) to analyze residual ash [22]. Removal of these metals from the final product often requires acid washing or other extensive measures, though this purification process comes with the less desired result of particle surface defects, so metals typically are not fully removed to balance preservation of particle structure with particle purity [24].

Residual metal content is of interest in several studies as these metals, particularly iron, are hypothesized to be drivers of toxicity through oxidative stress pathways. The Fenton reaction is often suggested as the generator of these free radicals and occurs when an iron particle is oxidized in the presence of hydrogen peroxide to produce superoxide or hydroxyl free radicals. Free radicals are then available to interact and interfere in numerous cell signaling cascades and have been associated with mitochondrial dysfunction, cancer and genotoxicity, disruption of macrophage activity and phagocytosis, and more [25-28].

One key study by Kagan et al. in 2006 investigated the potential for toxicity outcomes associated with residual iron catalyst in SWCNTs [26]. Two materials were tested. First was the as-produced SWCNT with an iron content of $26 \%$ by weight, and the second was the same material following acid 
washing to purify the product with an iron content of only $0.23 \%$ by weight remaining. This study demonstrated that higher iron content did induce greater hydroxyl free radicals in an acellular model, though when applied within a RAW 264.7 macrophage in vitro model, no superoxide radicals could be induced by neither the purified nor the as-produced particle suggesting limited interaction of the metals with macrophages. In another study by Pacurari et al. (2012), MWCNTs were capable of inducing epithelial cell permeability, macrophage activation and migration, and actin filament remodeling though mechanisms dependent upon oxidative stress responses [28].

In a recent study by Lee et al. (2020), MWCNTs with various metal content, including Mitsui-7, were assessed for their iron content, other metal impurities, and their ROS generating abilities [29]. This was followed with an assessment of inflammation in rat lungs at one day post-intratracheal exposure to MWCNTs. This study found that the soluble iron content alone, nor the ROS generating potential of the materials correlated with inflammation, the total transition metal content may serve as a predictor of MWCNT induced inflammation. However, this study also noted that particle size may be more important as a predictor than metal content, though together these characteristics may be useful in predicting toxicity outcomes.

Other studies have also demonstrated limited evidence of CNTs inducing free radical production or oxidative stress in both in vitro and in vivo systems, and even suggest that CNTs can act as free radical scavengers [30-32]. Further research may be necessary to clarify the role of CNT/Fs in the generation of oxidative stress and the possible mechanisms involved.

\subsubsection{Functionalization}

Functionalization is an alteration to the surface chemistry of CNT/Fs in order to alter their reactivity or dispersion to optimize performance for a particularly intended application. Often, this functionalization is designed to improve dispersion within a matrix to produce a final, cohesive composite 
product. Furthermore, this change in surface chemistry can modify its interactions with other molecules to improve adherence and incorporation into its intended matrix or system of application. While these modifications are often made in consideration of their intended final product, this functionalization may also alter their interactions with biological systems following exposure.

Several key studies have attempted to investigate the role of CNT/F functionalization in relation to toxicity outcomes. In a series of studies by the NRCWE (Denmark), CNTs with various surface functionalization were tested including hydroxylation and carboxylation [11, 12]. However, the functionalization was found to not always be present as reported by the production facility, suggesting that certain modifications may have a limited time before deterioration of this surface modification. For this reason, minimal conclusions could be drawn linking surface functionalization to toxicity outcomes.

Of note, one key question yet to be fully addressing within the literature is the stability of functionalization over time. This functionalization may degrade during a product's "shelf life" which may result in interlaboratory variations depending on experimental time-dependent factors. Additionally, once within the body or biological system, the stability of functionalization cannot be accurately determined. Functionalization may be quickly altered or degraded within bodily fluids with various levels of acidity or protein compositions. Overall, conflicting evidence for the role of functionalization in determining toxicity outcomes creates the need for further research.

Other studies have been more successful in linking functionalization to toxicity outcomes. In a study by Hussain et al. (2016), MWCNTs functionalized with hyaluronic acid were found to induce less pulmonary inflammation, less fibrosis, and less mucosal cell metaplasia than non-functionalized MWCNTs using both in vivo and in vitro models, suggesting that the interactions of CNTs within biological systems can be altered with various surface modifications [33]. One other key study by Landry et al. (2016) investigated whether surface functionalization can alter their degradation, and therefore biopersistence, of MWCNTs in an in vitro model. This study found that particle length may also be an 
important contributing characteristic to biopersistence, though functionalization may be protective against macrophage degradation, therefore increasing the particle's biodurability [34]. Also, in a study by Wang et al. 2014, as-produced MWCNTs and MWCNTs functionalized with -COOH groups were assessed for their fibrogenic potential. This study reported that functionalization resulted in less activation of TGF $\beta$ activation and less fibrosis in both a C57 mouse model and in vitro in a human epithelial cell line [35]. Other studies highlight that functionalization alone is insufficient and other factors must be considered such as purification [36].

These findings suggest that functionalization with hyaluronic acid or carboxylation may play a role in predicting toxicity outcomes, and that functionalization with carboxylation may reduce toxicity outcomes and demonstrate that surface functionalization may both increase or decrease a particle's biopersistence and therefore toxicity outcomes depending on the state of altered biological interactions.

\subsubsection{Summary}

These physicochemical characteristics have been highlighted due to their historically noted impact in influencing toxicity outcomes. Additional characteristics can also be considered including surface area, density, rigidity, polyaromatic hydrocarbon contamination, and surface charge. The subsequent studies included in later chapters include an in-depth characterization for all materials investigated in order to elucidate the potential link between physicochemical outcomes and their ability to provoke specific toxicity outcomes.

\subsection{Four Primary Toxicity Outcome Categories}

Four categories of toxicity outcomes can be highlighted in consideration of CNT/F toxicities and include genotoxicity, inflammation, pathology, and systemic translocation. While these outcomes are often interlinked with one another, each one stands as an individual consideration regarding adverse health 
effects. Since the early 2000s, researchers have investigated these outcomes in both in vivo and in vitro modeling systems with a wide variety of experimental approaches, and a selection of highlighted studies can be found in the following discussion below.

\subsubsection{Genotoxicity}

The genotoxic potential and carcinogenic properties of CNT/Fs are still greatly unexplored. In 2014, the International Agency for Research on Cancer (IARC) classified one material in particular, Mitsui-7, as possibly carcinogen to humans (Group 2B), while all other CNT/Fs were categorized as not classifiable (Group 3), indicating that there is not enough evidence to make a definitive conclusion regarding their carcinogenicity. In the 2020-2024 Report of the Advisory Group to Recommended Priorities for the IARC Monographs, MWCNT were listed as a high priority to be ready for evaluation within five years [37]. This report identified a key knowledge gap in understanding the toxicities of $\mathrm{CNT} / \mathrm{Fs}$ and encouraged research teams to further investigate CNT/Fs for their genotoxic potential in order to more accurately assess and categorize these materials.

To date, the carcinogenicity of CNT/Fs has been minimally investigated, however, several key studies can be noted. Takagi et al. in 2008, and again later in 2012, exposed p53(+/-) mice to Mitsui-7 via intraperitoneal injection (i.p) and quantified tumor occurrence and mortality[38, 39]. These studies found that mice exposed to Mitsui-7 developed mesothelioma in a dose and time dependent manner. Furthermore, several studies using rat models, including F344, Wistar, and F334/ Brown Norway hybrid rats, found that i.p injections or intra-scrotal injections of MWCNTs, including Mitsui-7 and other MWCNTs of various sizes and physicochemical properties, could also induce mesothelioma [14, 40, 41]. While these studies demonstrated the capabilities of MWCNTs to be carcinogenic, one key limitation was the method of dosing. It was not until 2014 and later that several studies specifically linked inhalation exposure to lung cancers. These studies found that inhalation exposure to MWCNTs, often using the 
aforementioned Mitsui-7, could result in increased tumor incidence and tumor burden compared to controls in both mice (B6C3F1) and rats (F344 and F344/Crj) [42-44]. Overall, these studies demonstrated the carcinogenic potential of MWCNTs, though most of these studies used the same infamous material.

While several of these studies linked exposure to tumor incidence, mechanisms of carcinogenicity are also under investigation. Several key mechanistic events have been highlighted as necessary for carcinogenicity and include disruption of the cell cycle, increased signaling for cell proliferation, oxidative stress, changes to the tumor microenvironment, and genotoxicity [7, 28, 45-47]. Genotoxicity is of particular interest in the following chapter. Genotoxicity is DNA damage resulting from direct and indirect mechanisms and includes mitosis disruption, chromosomal damage, and impaired DNA repair processes. Several studies have noted these mechanisms following CNT exposure. In 2014, Siegrist et al. reported that exposure to MWCNTs resulting in damage to the mitotic spindle as well as increase in aneuploidy, or abnormal chromosomal numbers, which are markers of disrupted mitosis [46]. Furthermore, this study reported cell cycle arrest, another marker for carcinogenicity. Another hallmark of genotoxicity, micronuclei formation (fragmentation of nuclear material), was reported by Muller et. al as early as 2008 and reported by others including Bishop et al. in 2017 [19, 48].

Together, these reports suggest that MWCNTs may be capable of inducing genotoxicity through various mechanisms, but additional studies are necessary to elucidate these critical pathways and to determine if all CNTs induce genotoxicity and carcinogenicity through the same mechanisms. Additional exploration of these potential pathways as well as studies comparing more CNT/Fs within the same study paradigm will be needed to fully understand the carcinogenic potential of CNT/Fs and properly classify the risks associated with particle exposure. 


\subsubsection{Inflammation}

Inflammation is one of the most well understood outcomes of CNT/F exposure. Many studies have demonstrated that pulmonary exposure to CNT/Fs can result in cellular injury and activation of inflammatory cascades. Endpoints such as lactate dehydrogenase (LDH) release, inflammatory cell infiltration, cytokine, and chemokine release, and inflammasome activation, as well as functional outcomes such as changes in macrophage phagocytic abilities, and changes in cell proliferation and transformation can be assessed. Several hallmark studies of CNT/F inflammation have been completed to date and are discussed below.

The first of these studies was completed in 2005 by Muller et al. in which Sprague-Dawley rats were intratracheally dosed with MWCNTs and subsequently underwent bronchoalveolar lavage (BAL) at 3- and 15-days post-exposure [49]. A dose dependent increase in LDH was observed. This classic marker of cellular injury can be found when cell membrane damage results in the release of this cytoplasmic enzyme into the extracellular space. Additionally, granulocyte accumulation as well as infiltration of eosinophils and neutrophils was observed. The infiltration of inflammatory cells including macrophages, eosinophils, neutrophils, and lymphocytes are also classic markers of inflammation. This study was one of the first to compare the pulmonary toxicities of MWCNT to that of asbestos and carbon black, as well as to consider the role of particle size in determining toxicity outcomes.

The second key study was completed in 2009 by Ma-Hock et al. and was a 90-day inhalation toxicity study using Wistar rats [50]. The particle used in this study was the Nanocyl 7000 MWCNT, and the authors reported increases in neutrophil infiltration and total protein content in bronchoalveolar lavage fluid (BALF). Furthermore, pathology assessments at 24 days following the last exposure reported inflammatory cell infiltration. This novel study was the first to implement inhalation dosing methodology to improve translation to human studies, and to investigate the effects of MWCNTs on various regions of the respiratory tract. 
Porter et al. (2010) is the third hallmark study of MWCNT inflammation [51]. C57 mice were exposed to Mitsui-7 via oropharyngeal aspiration. This was also the first study to complete a thorough dose $(10,20,40,80 \mu \mathrm{g})$ and time course $(1,7,28$, and 56 days post-exposure) study of MWCNT induced inflammation. This study reported that while inflammation seems to peak around 7 days post-exposure as measured in inflammatory cell infiltration, cell counts remained consistently elevated compared to controls at the latest time point. Similar trends were also reported for LDH and albumin in the BALF. Furthermore, dose-dependent effects were also observed at all time points in which greater doses of MWCNT resulted in greater inflammation. This study was one of the first to report the pulmonary toxicities of Mitsui-7, as well as to investigate inflammation in relation to dose and time.

Mercer et al. in 2013 published a 12-day inhalation study that investigated the long-term effects of MWCNT inhalation [10]. C57 mice were exposed to Mitsui-7 and inflammation, as well as a thorough investigation of fibrosis, was assessed at 1, 14, 84, 168, and 336 days post-inhalation, This study reported that inflammatory BALF cell infiltration and LDH was significantly elevated at 168 days post-exposure, with slight, but not statistically significant, elevation at 336 days post exposure. This was one of the first to highlight the chronic inflammatory activation of MWCNTs, but to also demonstrate that inflammation will subside over time when exposures cease.

In 2014, Sweeney et al. published a study investigating the effects of MWCNT on alveolar macrophages [52]. Primary human alveolar macrophages were exposed to $10 \mu \mathrm{g} / \mathrm{ml}$ long and short MWCNTs in vitro for 24 hours. Inflammatory cytokine release was investigated, and the authors report that IL-6 release was significantly increased following treatment with the longer of the two MWCNTs. Furthermore, functional changes in phagocytic capacity were assessed and found that both long and short MWCNTs could significantly reduce the phagocytic abilities of macrophages, which has implications for altered biopersistence of particle in the lung, as well as clearance and extrapulmonary translocation of 
tubes. This study is also notable for its contributions of linking physicochemical characteristics to toxicity outcomes.

A couple notable papers have been published regarding the toxicities induced by carbon nanofiber exposure. First is the study by Delorme et al. in 2012 in which Sprague Dawley rats were exposed to CNF by inhalation for up to 90 days [53]. Cell damage, inflammation, and histopathology were assessed. This study reported sub-chronic inflammation as well as notable histopathological changes including pulmonary fibrosis that persisted through later time points in a dose-dependent manner.

A second CNF hallmark study was published in 2018 by Hamilton et al. which investigated the role of CNFs in inflammation and the activation of the inflammasome [54]. Alveolar macrophages, both mouse and human, were exposed to up to $50 \mu \mathrm{g} / \mathrm{ml} \mathrm{CNF}$ and evaluated for various markers of inflammasome activation including LDH, IL-1 $\beta$, IL-18, and cell viability and proliferation. Additionally, C57BL/6 mice were exposed to $40 \mu \mathrm{g}$ CNF via aspiration. Markers for inflammasome activation including LDH and cathepsin B were assessed in BALF. This study found that NLRP3 inflammasome activation was present in all models used. This study is important for its notable use of CNF and for the investigation of mechanisms behind the previously observed inflammatory responses, including the activation of the inflammasome.

In conclusion, these studies through the years have shown that $\mathrm{CNT} / \mathrm{F}$ are capable of inducing potent activation of pulmonary inflammation. Cellular injury, activation of the inflammasome and other cellular signaling cascades, and changes in phagocytic abilities can have persistent impacts and can serve as drivers in relation to other toxicity outcomes including, histopathology alterations and translocation and particle biopersistence. The question still remains if all CNT/F have the same inflammatory potential as all other $\mathrm{CNT} / \mathrm{F}$, or if the cross-linked outcomes are all equally affected by changes in inflammation. Future studies are still necessary to answer overarching questions regarding the toxicities of the broad class of materials.] 


\subsubsection{Pathology}

Numerous histopathology changes can be observed in the lung following exposure to nanoparticles that can be associated with changes in morphology of the tissue, inflammation, and changes in functional respiratory outcomes. Several of these outcomes are discussed in detail.

\subsubsection{Granulomatous Inflammation}

Pulmonary exposure to MWCNT is known to induce granulomatous inflammation or granuloma formation with associated fibrosis. This granulomatous response is predicted to be the result of frustrated phagocytosis or inability to clear the particle from tissue. In order to prevent persistent tissue injury and activation of an inflammatory response, CNT/Fs and CNT/F agglomerates are enclosed in a granuloma. A granuloma is an encapsulating wall composed of immune cells, such as macrophages, neutrophils, and lymphocytes, which serve as a barrier between the particle and healthy lung tissue thereby preventing further cellular injury. Macrophages involved in these lesions are often giant, or multinucleated, or are observed to have granular cytoplasm [55]. Granulomas often contain collagen deposits for the long-term stability of the encapsulation and are generally considered to be permanent.

Minimal presence of granulomas can have little to no adverse health effects. However, with increasing severity, number, and size of granulomas, diminished respiration and elasticity of lung tissue can occur. While granulomatous lung disease if often associated with both infectious and non-infectious causes including tuberculosis and sarcoidosis, nanoparticle driven granulomatous response has been observed. Few studies have attempted to quantify functional changes in respiration following CNT/F exposure in rodents, though histopathological outcomes often consider granuloma formation. Furthermore, to date, no human studies have reported functional changes in respiration as an adverse health outcome nor have histopathological outcomes been assessed to observe changes including fibrosis as the latency for these changes has not yet been achieved. 
While granulomatous inflammation is often seen following CNT/F exposure, a particular note should be made considering the route of exposure. Well-dispersed CNT/Fs dosed via inhalation methods may not result in heavy particle or large agglomerate deposition in the lung and may not induce a granulomatous response compared to other methods of bolus-type dosing such as oropharyngeal aspiration [10]. The translational aspect of bolus-type dosing should be considered when extrapolating rodent findings to human health outcomes [15].

\subsubsection{Small airway disease and pathology}

Particle induced small airway disease has been previously characterized following exposure to mineral dusts, air pollution, and other fine particulates such as silicates and metal oxides. As these particles are frequently deposited in the bronchi and bronchioles, pathological changes are frequently observed including hypertrophy and hyperplasia, bronchiolitis obliterans-like changes, and fibrosis. Hypertrophy and hyperplasia of the bronchial epithelium is a thickening of the epithelial layer of cells as both the number of cells and cell layers present increase and the size of the epithelial cells themselves increases [55]. This thickening occurs as a response to cellular damage and can occur with and without the activation of inflammatory cascades and may also be linked to oxidative stress. Furthermore, bronchiolitis obliterans like changes have been previously observed and are known to occur when a combination of pathology changes in the bronchi and bronchioles results in a constriction of the small airways leading to potential respiratory difficulties.

In a previous study investigating MWCNT exposure in rodents, MWCNTs that heavily deposited in the small airways resulted in the occurrence of bronchiolitis obliterans-like changes, suggesting that the particles themselves may serve as a direct driver of these pathologies [19]. Future research into these exact mechanisms are still necessary. While the latency for human studies of pathology changes has not been 
reached, the additional use of rodent models can be an acceptable system for investigating these potential outcomes.

\subsubsection{Pathology of the alveoli and gas exchange regions}

Similar to the pathology changes in the small airways, changes in the alveoli region of the lung have also been observed following exposure to particulates, and several studies have investigated the potential effects of CNT/Fs. Additionally, similar to observations in the bronchi, hyperplasia has been

observed in the alveoli. This hyperplasia is recognized as an increase in alveolar epithelial type II cells and increased cell number, or hypercellularity [55]. Furthermore, alveolar histiocytosis, or an accumulation of macrophages often presenting with foamy cytoplasm within the alveolar region, may also occur following CNT/F exposure and is a hallmark of inflammation $[19,55]$. These lesions are classic indications of persistent cellular damage and inflammation.

\subsubsection{Pulmonary Fibrosis}

Studies have shown that exposure to MWCNT can result in fibrosis. Stimulation of resident fibroblasts will result in increased collagen production and will be readily observed as excessive or abnormal deposition of collagen with special stains such as Masson's trichrome stain or picrosirius red can result in stiffening and decreased elasticity of lung tissue and the increased thickness can increase the distance across where gas exchange occurs resulting in difficulty expanding and contracting the lungs and diminished gas exchange, respectively[9, 55]. Additionally, highly affected regions may also result in atelectasis, or focal alveolar collapse.

Two distinct regions for pulmonary fibrosis are of interest for CNT/F exposure. First, bronchi and bronchiolar fibrosis can occur the bronchi and bronchiolar epithelium. This thickening can occur when particle deposits in these regions and stimulates fibroblast activity. Due to the branching structure of the 
terminal bronchioles and the broncho-alveolar duct, combined with their narrowing diameters, particle is likely to deposit within this region leading to fibrosis. Bronchiolitis obliterans-like obstructions are also commonly seen in conjunction with this fibrosis leading to the obstruction and impaired respiration in downstream airways and alveoli. Duke et al. published a study in 2017 noting that MWCNTs that deposited in the bronchiolar region induced a potent fibrotic response in the small airways of mice exposed to rod-like MWCNTs at 21 days post-exposure that resulted in airway constriction [56]. Similar findings were reported in 2017 by Bishop et al. in which mice exposed to large tangles of MWCNTs that deposited in the small airways resulted in bronchiolitis obliterans-like pathologic alterations [19].

Furthermore, the respirable fraction of CNT/Fs can reach the alveolar ducts. As previously mentioned, these particles can readily diffuse into the alveolar interstitium resulting in fibroblast stimulation and subsequent collagen deposition. This thickening of the alveolar interstitium can increase the thickness of tissue, resulting in a greater distance across where gas exchange must occur. Severe alveolar fibrosis can lead to poor respiration. Several studies have noted the occurrence of alveolar fibrosis. In 2010 Porter et al. completed a dose and time response study to measure pulmonary injury following exposure to MWCNT and determined that the fibrotic response was measurable as early as 7 days post-exposure that persists through chronic time points [51].

Overall, pulmonary fibrosis is of particular concern following CNT/F exposure. Additionally, fibrosis was of importance in the determination of the NIOSH recommended exposure limit (REL). This REL was based upon the likelihood of developing fibrosis (early stage lung disease) as an adverse health effect at a likelihood of $0.5-16 \%$ [57]. This outcome, in combination with genotoxicity outcomes, are of key consideration in any future re-evaluations of the REL as well as establishing safe workplace handling procedures. 


\subsubsection{Pleural penetrations}

Pleural penetrations are a hallmark of tissue injury often associated with asbestos exposures and have been linked to mesothelioma outcomes. Previous studies have shown that particle dosing directly to the pleural space can result in mesothelioma due to injury to the mesothelial cells present $[14,38,39,41]$. Therefore, particle penetrations into the pleural space are a linked as a potential driver of particle induced lung cancers and inflammation.

Several studies have also noted pleural penetrations following exposure to MWCNTs, particularly following inhalation of the well-known Mitsui-7 MWCNT. Porter et al. in 2010 were the first to report pleural penetrations as early as 12 days post exposure in mice [51]. In 2010, an additional study by Mercer et al. used a pharyngeal aspiration model in mice to quantify the pleural penetrative capabilities of Mitsui7 [58]. This study found that penetrations occurred quickly and were seen at one day post exposure, though these penetrations may have been subsequently cleared via macrophage mediated clearance. However, increasing deposition of tubes in the pleural and subpleural tissue increased and accumulated as early as 28 days post-exposure.

\subsubsection{Translocation}

Extrapulmonary translocation to other organs including lymph nodes, liver, spleen, and kidneys have been noted in various studies [59-61]. As previously indicated in the lung, the location of particle deposition is important as many adverse health effects are related to the direct presence and interaction between particle and tissue. While systemic outcomes may or may not be related to the physical presence of tissue, the accumulation of particle in extrapulmonary tissue is an important consideration for systemic toxicity outcomes $[62,63]$. Due to possible mechanisms driven by the "systemic spill-over" effect in which inflammation signals from the lung reach systemic circulation, extra-pulmonary inflammatory cascades may be activated even in distant tissues, without the presence of accumulated particle [62]. 
This translocation can occur through two keys mechanisms. First, independent, passive particle translocation can occur without intervention of the immune or lymphatics system. Due to the hydrophobic nature of CNT/Fs as well as their pointed ends, these particles can pierce and diffuse through cell membranes allowing particles to passively enter into systemic blood circulation. Once within systemic circulation, these tubes can eventually migrate to other organs, particularly the liver due to its high perfusion, where particles can deposit and accumulate over time. Minimal translocation has been previously reported to extrapulmonary tissues, those these percentages are often minimal ranging from tenths to hundredths of a percentage of the original lung burden [64].

Second, macrophage mediated translocation occurs when macrophages and monocytes are capable of phagocytosing particles and subsequently migrating to other regions of the body, primarily through the lymphatic system. Within the lung, two notable regions for lymphatics flow can be identified. Lymphatic flow can be easily found intermingling at the bronchoalveolar duct region and terminal bronchioles. This area is also identified as a location of large blood vessels and vascularization. Second, the pleural space, or the region between the outer wall of the lung and the inner wall of the thoracic cavity is also a region of high lymphatic flow. Macrophage mediated translocation to the pleura was first reported by RymanRasmussen et al. in 2009 following inhalation of CNTs in mice [65]. Each of these regions will continue flow to the local lymph nodes. Once in the lymph nodes, these particles can often be ensnared and migrate from encapsulation in the macrophages to within resident cells of the lymph nodes. Like other organs, these particles are likely to accumulate over time with recurrent exposures.

Several studies of note demonstrate this particle migration and extrapulmonary accumulation following inhalation or pulmonary exposure to CNT/Fs. In 2010, Reddy et al. dosed male Wistar rats with CNTs via intratracheal instillation [66]. This study reported extrapulmonary translocation to the kidney and liver with associated toxicities. In 2013, Mercer et al. published two studies investigating the fate of inhaled MWCNTs in a mouse model $[10,64]$. The first study found that a significant portion of MWCNTs 
could be phagocytosed by macrophages in attempted particle clearance. The second study specifically investigated extrapulmonary translocation of MWCNTs. Translocation to the lymph nodes was common and accounted for over $7 \%$ of the initial lung burden of particles at one-year post-exposure. To a lesser extent, particle was also found in the liver, kidneys, heart, brain, chest wall, and diaphragm. This study also notes that particle accumulates in tissue over time. In an inhalation study using mice by Ingle et al, also in 2013, Raman spectroscopy was used to follow particle translocation and found that particle can translocate via the bloodstream where it can deposit in extrapulmonary tissues [67]. These three studies together demonstrate that particle can translocate to extrapulmonary tissues via macrophage-mediated transport and independent flow via the bloodstream where the particle can deposit and accumulate in numerous other tissues. Further research is still necessary to elucidate the role of these particles directly on systemic outcomes and to differentiate these direct effects from potential indirect effects of particle exposure.

\subsubsection{Summary}

Overall, these four outcomes highlight the range and extent of health outcomes that can be altered by pulmonary exposure to CNT/Fs. These outcomes can be readily assessed using in vitro and in vivo model systems, though human data of these four outcomes is lacking. The latency for human research has not yet been achieved for the assessment of most outcomes such as genotoxicity and fibrosis, and, to date, few human studies have been completed.

\subsection{Risk, Exposure, and Current Human Studies}

\subsubsection{Current Occupational risks, guidelines, and exposure prevention methods}

Risk is the likelihood of detrimental effects to occur in a given scenario or event, and the present study is critical to understanding the risk of harmful effects following exposure to nanomaterials, 
particularly CNT/F. Risk is a combination of two factors, the likelihood of exposure and the severity of the potential adverse outcome. Minimizing the risk associated with $\mathrm{CNT} / \mathrm{F}$ exposure is primarily dependent upon reducing exposure to particles. During production of $\mathrm{CNT} / \mathrm{F}$, facility employees can be exposed to dry powder materials that can be aerosolized and respired. Once in the lung, these materials may induce the toxic outcomes previously discussed. These effects can cause irreversible pulmonary and systemic effects that can lead to diseases such as cancer or pulmonary fibrosis.

In 2013, NIOSH published the Current Intelligence Bulletin 65: Occupational Exposure to Carbon Nanotubes and Carbon Nanofibers in which they established a recommended exposure limit of $1 \mu \mathrm{g} / \mathrm{m}^{3}$ elemental carbon as a time weighted average for an 8-hour workday [57]. While there is no policy enforcement of this recommendation, this limit is strongly urged to be upheld by primary and secondary manufacturing facilities in the United States. In a recent assessment of U.S. facilities, $93 \%$ of respirable measures across 12 facilities were below the REL of facilities maintained exposures at or below the NIOSH REL [68]. These standards are derived from risk assessment studies that link studies of human exposures with non-human studies including rodent studies in order to predict the threshold at which human adverse outcomes are likely to occur.

In order to minimize risk, several steps can be taken to protect workers. The first step is elimination and substitution to nullify the possibility of exposure. However, emerging nanomaterials, including $\mathrm{CNT} / \mathrm{F}$, are often on the cutting edge of technology and substitutions or eliminations are not feasible. The second step is the use of engineering controls to reduce exposure. These engineering controls include the use of personal protective equipment such as respirators, protective eyewear, and gloves to minimize respiration as well as alternative exposure routes such as dermal exposure. Engineering controls such as air filtration can also be used to minimize exposures. Lastly, secondary prevention measures can be taken to assess outcomes and consequences of exposures. Investigations including epidemiological studies and medical surveillance can be used to detect human health consequences early. These methods together can 
be used to minimize worker exposures as well as monitor those who are exposed for adverse health outcomes allowing for early intervention if necessary. These steps are critical for the safety and protection of CNT/F facility workers.

\subsubsection{Collaborative exposure assessments}

Exposure assessments are studies completed to quantify human exposures in the workplace as well as identify critical means or methods by which workers are most likely to be exposed. These studies can determine if workers are exposed to levels below recommended exposure limits and determine the respirable and inhalable fractions of CNT/F. Additionally, these studies can be used to determine which steps in the manufacturing process are most likely to lead to exposure.

Several human exposure assessments have been completed. In one key study and follow up study,

14 U. S. facilities were assessed including companies that produce CNT/F, use CNT/F in secondary production such as the production of composites that contain $\mathrm{CNT} / \mathrm{F}$, or a hybrid company that produces both $\mathrm{CNT} / \mathrm{F}$ and the secondary product $[68,69]$. In these studies, samples of the personal breathing zones (PBZ) for facility employees were collected to measure employee exposure to CNT/F. It was determined that these facility employees were exposure to levels below the NIOSH REL for the respirable fraction, but not the inhalable fraction, and PBZ samples primarily contained particle agglomerates greater than $2 \mu \mathrm{m}$ in diameter. This study raised concerns about the toxic effects of these particles as agglomerates as well as the importance of distinguishing the respirable and inhalable fractions. Of note, these studies have been paired with the final series of human health effects studies discussed below, and with the studies to follow in subsequent chapters.

\subsubsection{Human health effect studies}


Only twelve key studies have been published to date that attempted to directly detect or quantify health effects following exposure to MWCNT/F in humans and have been generally completed by five main groups. The first of these were completed by Liou et. al in 2012, and a second follow up study, also by Liou et al. (2014) was subsequently published[70, 71]. This study investigated human health effects from employees of Taiwanese facilities that produce various nanomaterials, and of note, only $23 \%$ of the total exposed employees in this study were exposed to $\mathrm{CNT} / \mathrm{F}$. Blood, urine, and exhaled breather condensates were collected from a total of 227 particle exposed employees and 137 control employees from fourteen difference facilities. This study noted significant decreases in superoxide dismutase (SOD) and glutathione peroxidase (GPx), in addition to increases in vascular cell adhesion molecule 1 (VCAM1) from baseline at a six-month follow-up. In the second publication reporting a four year follow up study, similar findings were reported in addition to an increase in cardiovascular biomarkers fibrinogen, IL-6, and intracellular adhesion molecule 1 (ICAM-1). No other significant findings were reported, including spirometry and functional outputs.

The second of these groups was published in 2015 by Lee et al. who competed a health surveillance study to quantify changes in blood and exhaled breath condensates of manufacturing employees [72]. This study was able to identify three biomarkers present in exhaled breath condensates: malondialdehyde, 4hydroxy-2-hexenal, and n-hexanal levels. However, no functional changes in the respiratory abilities of the employees were detected, and this study was limited by the number of participants ( $\mathrm{N}=9$ exposed employees, and $\mathrm{N}=4$ non-exposed).

The third series of publications were completed in 2016 by Fatkhutdinova et al. and Shvedova et al. $[73,74]$. The first study published quantified inflammatory markers in the blood and sputum of MWCNT manufacturing facility employees and found an increase in markers including IL-1B, IL-4, and TGF- $\beta$ in both blood and sputum and additional increases in IL-5, IL-6, Il-8, and KL-6 in sputum. The second publication assessed changes in non-coding RNA (ncRNA) and messenger RNA (mRNA) in the 
blood of exposed facility employees and found significant changes in pathways related to cell signaling, proliferation, apoptosis, as well as cardiovascular regulation. These findings suggested possible inflammatory, carcinogenic, and systemic adverse effects may occur. However, these studies were also limited by small sample numbers ( $\mathrm{N}=8-10$ exposed, $\mathrm{N}=7-12$ non-exposed employees).

The fourth series of publications were published between 2016 and 2018 by Vlaanderen et al., Ghosh et al., and Kuijpers et.al. [75-77]. These studies also relied on a relatively smaller sample number ( $\mathrm{N}=22-24$ exposed workers and $\mathrm{N}=39-42$ non-exposed employees). These studies also attempted to complete follow-up assessments of the same workers to identify changes over time, though this group was much smaller, and included ten-thirteen exposed and four-six non-exposed employees. In these studies, immunological, respiratory, and circulating blood factors were analyzed for potential biomarkers and changes in lung function as a consequence of respiratory exposure to MWCNTs. In the first, Vlaanderen et al., inflammatory markers in the blood, lung function tests, and fractional exhaled nitric oxide (FENO) were assessed [77]. Only a few markers assessed had any significant changes and included c-c motif ligand 20 (CCL20), soluble interleukin-1 receptor type II (sIL-1RII), and basic fibroblast growth factor were increased, though control levels were quite variable. These markers also exhibited further changes in the follow up study, though again, this was limited by small sample numbers. No functional respiratory (spirometry) changes were observed, though FENO levels were significantly decreased in exposed workers. Lastly, some other hematological changes were observed including decreased neutrophil counts and increased monocyte counts in the exposed workers. Increased immature platelet counts and reticulocyte fractions were also observed in exposed workers, though no other significant changes in the blood samples were found.

The second publication by Ghosh et al. attempted to detect epigenetic changes in works exposed to MWCNTs by investigating global DNA methylation and hydroxymethylation [75]. While numerous genes were assessed for these changes, only a few sites had significant changes in methylation compared 
to non-exposed controls. These significant changes included DNA methyltransferase 1 (DNMT1), ataxia telangiectasia mutated (ATM), SKI proto-oncogene (SKI), and histone deacetylase 4 (HDAC4) promoter, which are genes that serve a role in the regulation of cell replication and proliferation, with potential links to carcinogenicity.

Last in this series is a publication by Kuijpers et al., that intended to investigate cardiovascular biomarkers associated with MWCNT exposure [76]. Of the twelve markers assessed, one marker, ICAM1, had significant changes in exposed workers in both the initial assessment and the follow-up assessment, similar to previously reported publications. Overall, these three publications attempted to investigate various outcomes of MWCNT exposure to consider inflammation, cardiovascular effects, and even links to cancer. While the sample number also remains quite small, particularly in the follow-up phase of the study, some interesting findings have emerged for consideration of human health effects.

The fifth human health effects study series was completed by close collaborators and are paired with the previously discussed exposure assessments and the studies found in the following chapters. In this series, over 100 exposed employees from 12 work sites were assessed, the largest sample number in any of the human health effects studies, and four publications have been made. The first study, Beard et al. 2018, exposed and non-exposed workers provided samples for biomarker analysis for a total of 37 blood and 36 sputum markers related to oxidative stress, fibrosis, inflammation, and cardiovascular responses [78]. Several sputum biomarkers were of note including matrixmetalloproteinase-2 (MMP-2), interleukin 18 (IL-18), GPx, SOD, and myeloperoxidase, and in blood, MMP-2, MMP-9, metalloproteinase inhibitor 1, GPx, SOD, fibrinogen, endothelin-1, ICAM-1, and VCAM-1, were noted.

The second study is the previously discussed exposure assessment from Dahm et al. (2018) in which it was reported that $18 \%$ of workers had CNTs within sputum samples, and $70 \%$ presented with dermal exposures [68]. The third study by Schubauer-Berigan et al. (2018) investigated functional respiratory and cardiovascular changes, allergic responses, and blood analytes [79]. Allergy responses 
were found to positively correlate with increasing years of employment in the facility as well as inhalable elemental carbon fractions. Additionally, systolic blood pressure and resting heart rate, as well as blood hematocrit, were noted to correlate to fine particulate matter, elemental carbon, and the number of CNT/F structures, respectively. However, most other factors analyzed, including spirometry measurements, had no significant outcomes, and the authors suggest the need for additional studies.

The most recent publication, also by Schubauer-Berigan et al. (2020), is the fourth publication in this series [80]. In this study, ex vivo methodology, a TruCulture assay was used to detect biomarkers from blood samples in response to stimuli. Regression modeling and pathway analysis was also used to determine the relationships between other contributing factors and the identify key signaling pathways that may have been affected or predictive of downstream health effects. Significant changes were observed for haptoglobin, IL-1b, IL-1ra, IL-3, IL-10, IL-12p40, IL-15, IL-18, IL-23, MMP-9, stem cell factor (SCF), tissue inhibitor of metalloproteinases 1 (TIMP1), vascular endothelial growth factor (VEGF), and von Willebrand factor. Furthermore, pathway analysis identified several cellular and molecular processes that may be influenced including cell-to-cell signaling and interaction, cellular growth and proliferation, cellular development, cellular movement, inflammatory response, immune cell trafficking and hematological system development and function. Overall, this study was able to highlight potential changes in human health effects that may not be readily detectable by simple biomarker analysis, suggesting undiscovered or currently unknown potential adverse human health outcomes.

\subsubsection{Summary}

Human studies have been limited by small sample sizes and minimal follow-up studies to assess changes over time, though some factors such as biomarkers of oxidative stress and inflammation have been identified. As previously mentioned, the latency for human exposures to present with adverse human health effects has not yet been reached, and many studies have attempted to identify predictive biomarkers 
and early changes in cellular and molecular pathways that may indicate potential outcomes. However, these limitations have resulted in a wide variety of outcomes between studies which may be influenced by other lifestyle factors that vary between cultures and geographic locations. Future research and continued monitoring are necessary to fully understand human health outcomes from CNT/F exposure. With improvements in safe handling practices and minimizing human exposure risk, the likelihood of significant and widespread effects can be minimized, though it remains critical to understand these possible effects through advancing in vitro, in vivo, and human studies.

\subsection{Conclusions and Justification of the Current Study}

While the toxicities associated with pulmonary exposure to MWCNTs and CNFs have been extensively studied, many questions are still prevalent. Are all CNT/Fs equally toxic, or should they all have the same risk classifications? In a recent call to revise the NIOSH CIB, attention has been drawn to the still prevalent knowledge gaps in the current understanding of the risks and adverse outcomes that may result from MWCNT/CNF exposure. Furthermore, the recent classification of CNTs as a high priority highlights the need for additional studies.

Current studies are often limited by their narrow focus on only a few materials such as the infamous Mitsui-7 MWCNT. Studies that do investigate other materials with the intention to compare them directly typically are limited to only a couple of particles. Even studies that attempt to compare a large variety of MWCNTs side-by-side still often fail to include a diverse enough representation of the whole class of MWCNT/CNFs such as size limitations. For example, the series of studies published by the NRCWE do include Mitsui-7 particle, though this particle is the largest included in the study, and at only $\sim 50-70 \mathrm{~nm}$ in diameter, a significant population of particle sizes were not considered. For this reason, future studies that are more representative of the large ranges of sizes and other characteristics are still needed. 
In addition to expanding the range of materials investigated, a multi-disciplinary approach may be necessary to elevate these studies to answer more complex questions regarding the relationships between physicochemical properties and the toxicity outcomes they may induce. This approach requires novel contributions from the fields of artificial intelligence and machine learning to elucidate relationships and meld the fields of engineering and biomedical sciences.

The expansive study in the following chapters attempts this more elegant and multi-disciplinary approach to further understand CNT/F toxicity outcomes and their potential drivers. This study hypothesizes that while all MWCNT/CNF are toxic, not all materials may induce the same toxicity outcomes, and these outcomes may be dependent upon the physicochemical characteristics of the material including, but not limited to, size dimensions, impurities and contaminants, density, dustiness and respirability, and agglomeration. Nine materials, seven MWCNTs and two CNF, were included in this study and encompass a large range of materials with diameters ranging from 9-150nm in diameter as reported by their respective production facility. This study also included Mistui-7 as a benchmark material. Eight of these materials are produced in U.S. facilities, the same facilities included in exposure assessments and human health effects studies previously discussed as completed by collaborators, therefore maximizing the potential for translational studies and conclusions.

In the first part of this study, these nine materials are extensively characterized and are assessed for their genotoxic potential in human bronchial epithelial cells (BEAS-2B). The second part of the study includes an in-depth investigation of the inflammation induced by these particles in vivo (C57 mouse model) at both acute and chronic time points ranging from 1-84 days post exposure. Additionally, mechanisms of inflammation are assessed in vitro (THP-1 macrophage) at a variety of doses at an acute time point. The third part of this study is a thorough assessment of the histopathological changes that can be induced in vivo at 84 days post-exposure as well as investigating the extrapulmonary translocation of these particle to tissues such as the trachea-bronchial lymph node and liver. In vitro assessments using 
human fibroblasts also attempt to investigate the implementation of the fibroblast model for the assessment of fibrosis and its possible mechanisms.

Within all three of these parts of the study, there is an additional assessment completed using machine learning to discover the relationships between the physicochemical characteristics and the toxicity outcomes. These correlations add additional layers to the conclusions to be drawn regarding the classification of particles, the relationship between in vivo and in vitro models, and the links between characteristics and outcomes. This multi-disciplinary approach expands upon the current understanding of $\mathrm{CNT} / \mathrm{F}$ toxicities and aims to eliminate the current unknowns within the field to improve knowledge of occupational risks, health outcomes, and contribute to safety-by-design. 


\title{
CHAPTER 2:
}

\section{Physicochemical Characterization and Genotoxicity of the Broad Class of Carbon Nanotubes and Nanofibers Used or Produced in U.S. Facilities}

\author{
Kelly Fraser ${ }^{1,2 *}$, Vamsi Kodali ${ }^{1,2 *}$, Naveena Yanamala ${ }^{1,2}$, M. Eileen Birch ${ }^{3}$, Lorenzo Cena ${ }^{4}$, Gary \\ Casuccio $^{5}$, Kristin Bunker ${ }^{5}$, Traci L. Lersch ${ }^{5}$, Douglas E. Evans ${ }^{3}$, Aleksandr Stefaniak ${ }^{6}$, Mary Ann \\ Hammer ${ }^{1}$, Michael L. Kashon ${ }^{1}$, Theresa Boots ${ }^{1}$, Tracy Eye ${ }^{1}$, John Hubczak ${ }^{1,2}$, Sherri A. Friend ${ }^{1}$, \\ Matthew Dahm ${ }^{7}$, Mary K. Schubauer-Berigan ${ }^{7,8}$, Katelyn Siegrist ${ }^{1,9}$, David Lowry ${ }^{1}$, Alison K. Bauer ${ }^{9}$, \\ Linda M. Sargent ${ }^{1}$, Aaron Erdely ${ }^{1,2}$
}

${ }^{1}$ Health Effect Laboratory Division, National Institute for Occupational Safety and Health, Morgantown, WV; ${ }^{2}$ West Virginia University, Morgantown, WV; ${ }^{3}$ Health Effects Laboratory Division, National Institute for Occupational Safety and Health, Cincinnati, OH; ${ }^{4}$ West Chester University, West Chester, PA; ${ }^{5}$ RJ Lee Group, Monroeville, PA; ${ }^{6}$ Repiratory Health Division, National Institute for Occupational Safety and Health, Morgantown, WV; ${ }^{7}$ Division of Field Studies Evaluation, National Institute for Occupational Safety and Health, Cincinnati, OH; ${ }^{8}$ International Agency for Research on Cancer, Lyon, France; ${ }^{9}$ University of Colorado Anschutz Medical Campus, Department of Environmental and Occupational Health, Aurora, CO

*These authors contributed equally to this work

Published in Particle and Fibre Toxicology on December 7, 2020

DOI: 10.1186/s12989-020-00392-w 


\subsection{Abstract:}

Background: Carbon nanotubes and nanofibers (CNT/F) have known toxicity but simultaneous comparative studies of the broad material class, especially those with a larger diameter, with computational analyses linking toxicity to their fundamental material characteristics was lacking. It was unclear if all CNT/F confer similar toxicity, in particular, genotoxicity. Nine CNT/F (MW \#1-7 and CNF \#1-2), commonly found in exposure assessment studies of U.S. facilities, were evaluated with reported diameters ranging from 6-150 $\mathrm{nm}$. All materials were extensively characterized to include distributions of physical dimensions and prevalence of bundled agglomerates. Human bronchial epithelial cells were exposed to the nine $\mathrm{CNT} / \mathrm{F}(0-24 \mu \mathrm{g} / \mathrm{ml})$ to determine cell viability, inflammation, cellular oxidative stress, micronuclei formation, and DNA double-strand breakage. Computational modeling was used to understand various permutations of physicochemical characteristics and toxicity outcomes.

Results: Analyses of the CNT/F physicochemical characteristics illustrate that using detailed distributions of physical dimensions provided a more consistent grouping of CNT/F compared to using particle dimension means alone. In fact, analysis of binning of nominal tube physical dimensions alone produced a similar grouping as all characterization parameters together. All materials induced epithelial cell toxicity and micronuclei formation within the dose range tested. Cellular oxidative stress, DNA double strand breaks, and micronuclei formation consistently clustered together and with larger physical CNT/F dimensions and agglomerate characteristics but were distinct from inflammatory protein changes. Larger nominal tube diameters, greater lengths, and bundled agglomerate characteristics were associated with greater severity of effect. The portion of tubes with greater nominal length and larger diameters within a sample was not the majority in number, meaning a smaller percentage of tubes with these characteristics was sufficient to increase toxicity. Many of the traditional physicochemical characteristics including surface area, density, impurities, and dustiness did not cluster with the toxicity outcomes.

Conclusion: Distributions of physical dimensions provided more consistent grouping of CNT/F with respect to toxicity outcomes compared to means only. All CNT/F induced some level of genotoxicity in human epithelial cells. The severity of toxicity was dependent on the sample containing a proportion of tubes with greater nominal lengths and diameters. 


\subsection{Introduction}

The evaluation of the potential toxicity of carbon nanotubes and nanofibers $(\mathrm{CNT} / \mathrm{F})$ began in the early 2000's [30, 49, 81, 82]. The general outcomes of toxicity studies to date indicated that pulmonary exposure to $\mathrm{CNT} / \mathrm{F}$ was capable of inducing inflammation, fibrosis, cancer, immunosuppression, and adverse cardiovascular and neurological outcomes in vivo [15, 42, 51, 53, 63, 83-88]. Studies of key importance also confirmed that certain $\mathrm{CNT} / \mathrm{F}$ were able to translocate from the lung to lung-associated lymph nodes as well as systemic tissues $[9,10,15,64,65]$. These results raised justifiable concerns regarding potential human health effects, especially in the occupational workforce, and prompted the need to design and conduct epidemiological studies. While the latency needed for clinical symptoms has not ended based upon other fiber toxicity models, as the average worker handling CNT/F has had just short of a decade of cumulative exposure, evidence suggests exposure-related effects primarily consisting of measures of inflammation, oxidative stress, and immunosuppression [70-73, 77-79, 89, 90]. The outcomes were generally mild with no consistent pattern of effect among studies. Evidence of CNT/F in the sputum was observed and a considerable number of workers, approximately $70 \%$, were subjected to dermal exposure [68, 79]. The National Institute for Occupational Safety and Health (NIOSH) established a recommended exposure limit (REL) of $1 \mu \mathrm{g} / \mathrm{m}^{3}$ as an 8-hour time-weighted average of respirable elemental carbon, a surrogate for CNT/F, following background correction for ambient elemental carbon [57]. Dahm et al. (2018) found that U.S. companies can, in fact, maintain the $1 \mu \mathrm{g} / \mathrm{m}^{3} \mathrm{REL}$, as $93 \%$ of respirable measures were below the REL from 214 collected samples at 12 different facilities [68], although historically, and globally, this has not always been the case [91]. More recently, potential adverse effects of the inhalable fraction, including airway fibrosis and bronchiolitis obliterans $[19,56]$, have been recognized. The inhalable fraction was often significantly greater than the respirable fraction by 4 times and $29 \%$ of the inhalable samples in U.S. facilities were greater than $1 \mu \mathrm{g} / \mathrm{m}^{3}[68]$. Recently, the International Agency for Research on Cancer (IARC) classified one multi-walled carbon nanotube 
(MWCNT), the Mitsui-7 or MWCNT-7, as possibly carcinogenic to humans (Group 2B) [44]. There was insufficient evidence to classify all other CNT/F. The 2020-2024 Report of the Advisory Group to Recommended Priorities for the IARC Monographs indicates MWCNT as a high priority to be ready for evaluation within five years [37]. In summary, 1) in vivo studies indicated a significant hazard potential of $\mathrm{CNT} / \mathrm{F}, 2$ ) evidence exists of human exposure and health effect, 3) exposure can be controlled at recommended levels, 4) reevaluation for carcinogenicity is imminent, and 5) recommendations to fill toxicity knowledge gaps by examination of a broader class of CNT/F was warranted.

Our group recently conducted a cross-sectional study to evaluate exposure and potential associated health effects in workers handling CNT/F [68, 78, 79, 89]. From these studies, which evaluated 12 different facilities, and the years of ongoing exposure assessment of more than 20 facilities [69, 92], it was clear that a wide variety of $\mathrm{CNT} / \mathrm{F}$ were being produced or utilized by primary and secondary manufacturers. The production of $\mathrm{CNT} / \mathrm{F}$ continues to increase, and new high-volume applications are being evaluated, especially in the construction sector. The global CNT market is expected to grow from approximately USD 4.5 billion to USD 10 billion by 2023 and USD 15 billion by 2026 with a compound annual growth rate of $16 \%$. The primary question arising from a commercialization, industrial hygiene, and research perspective was whether all as-produced CNT/F materials confer similar toxicity. In controlled studies, differing physicochemical characteristics of CNT, such as length, diameter, functionalization, or surface coating in turn altered the in vivo pulmonary toxicity profile $[6,12,14,19$, 33, 93-100]. To date, very few studies simultaneously compared a broad class of as-manufactured CNT and linked the relationship between physicochemical characteristics and toxicity endpoints.

In this current series of studies, with guidance from extensive facility exposure assessment [68, 69, 92], we selected six MWCNT and two carbon nanofibers (CNF), collectively termed CNT/F, either manufactured or handled by U.S. companies, to evaluate four primary parameters of toxicity using in vitro and in vivo studies. Specific CNT/F types were selected to be broadly representative of those to which 
U.S. workers may be commonly exposed. The parameters included genotoxicity, inflammation, pathology, and extrapulmonary translocation. CNT/F selection was initially based on provided company diameter. Nominal tube diameter was the simplest way to delineate samples for testing and previous studies indicate a changing toxicity profile with increasing diameter (or rigidity) $[9,12,93,101]$. The selected materials ranged from 6-150 $\mathrm{nm}$ in diameter according to company specifications. Determining the materials to test according to diameter, other key physicochemical characteristics also were expected to vary, such as length $(5-200 \mu \mathrm{m})$, thus providing a proper representation of the CNT/F material class. A seventh MWCNT, Mitsui-7/MWCNT-7, was added as a benchmark material given the IARC carcinogenicity classification and the large amount of historical toxicity data available for the four parameters of interest. Of the materials selected, four MWCNT had reported company diameters smaller than the benchmark material, and two MWCNT and two CNF had diameters larger than the benchmark material. In the few comparative studies that examined multiple different materials, the larger diameter materials were not evaluated $[12,93,94]$.

For this section of the evaluation of CNT/F toxicities, all materials were extensively characterized, and genotoxicity, one of the four primary parameters of toxicity, was evaluated in vitro. Analyses included physical dimension, residual metal catalysts, dustiness, density, charge, acellular reactivity, surface area, endotoxin and PAH impurities, thermogravimetric analysis, and hydrodynamic diameter in suspension. Prevalence and forms of bundled agglomerates were also characterized as exposure assessment indicated that agglomerates, not singlets or individual fibers, represent the majority of particles in personal breathing zone samples in workplaces [92]. Human bronchial epithelial cells were treated with CNT/F to determine cell viability, inflammation, oxidative stress, micronuclei formation, and DNA double-strand breakage. Computational modeling was applied to physicochemical characteristics alone, and in conjunction with toxicity outcomes. The modeling created clustering by material, as well as response, to evaluate the relationship between physicochemical characteristic(s) and various toxicity endpoints. 


\subsection{Results and Discussion:}

\subsubsection{Physicochemical Characterization}

Seven MWCNT and two CNF (CNT/F), were arranged according to their diameter as reported by the production facility and are referred to as MW \#1-7 and CNF \#1-2 (Figures 1 and 2). The arrangement was designed as the information was readily available from the company and selecting a wide diameter range was necessary to ensure representation of this large class of materials. Furthermore, one material, MW \#5, also known as Mitsui-7/MWCNT-7, has been commonly studied and was used as a benchmark material for comparison. All CNT/F were extensively characterized as detailed in Tables 1-3 and Figures $1-5$.

The typical representation of bundled agglomerates containing tubes/fibers with smaller diameters materials and transitioning to more elongated bundles with tubes/fibers of increasing diameter was readily observed (Figure 1). Also observed was the range in dimensions that could be present in each sample. For example, MW \#2 was a unique material containing two main populations, one with singlets or agglomerates of discrete tubes and the other having highly entangled, cross-linked MWCNT with an average diameter of $7 \mu \mathrm{m}$ and length of $48 \mu \mathrm{m}$ as measured by electron microscopy. In contrast, MW \#7 had a highly mixed population of diameters that ranged from very thin to very thick with diameters ranging from 9-425 nm (Table 1). All CNT/F were extensively characterized and detailed in Tables 1-3 and Figures 1-5. Of the studies that have simultaneously examined a broad class of CNT/F, a greater proportion of those materials were of diameters at or below MWCNT-7 (mean $=67 \mathrm{~nm})[12,93,94]$. We aimed to extend those studies by encompassing MWCNT with larger diameter tubes and, additionally, by including CNF. 


\subsubsection{Nominal tube physical dimensions}

The classic fiber paradigm links fiber dimensions and biopersistence with toxicity outcomes. Fibers have been defined by an aspect ratio, or the ratio of particle length to diameter (or width), greater than $3: 1$ with a length greater than $5 \mu \mathrm{m}$ and a diameter less than $3 \mu \mathrm{m}[15,102]$. Historically, length, more so than diameter, has been the key consideration in understanding the toxicities induced by high aspect ratio materials. In comparative studies, longer fiber lengths were often associated with greater toxicities of naturally occurring or synthetic fibers [41, 103-112]. Often, materials greater than $5 \mu \mathrm{m}$ in length were associated with the development of mesothelioma, greater pulmonary biopersistence and particle retention, and greater inflammatory and fibrotic responses. While longer fibers generally confer greater toxicity, short fibers, those less than $5 \mu \mathrm{m}$ in length, are not without toxicity [113].

The comparison of high aspect ratio CNT/F to asbestos was a natural progression $[7,15,114,115]$. Several comparative studies assessing the effects of length and development of mesothelioma indicated that $\mathrm{CNT/F}$ may have similar capabilities to induce adverse effects. General consensus among the literature indicates that longer $\mathrm{CNT} / \mathrm{F}$ particles were more likely to activate downstream inflammatory cascades, induce fibrogenesis, interrupt macrophage clearance, and were generally more bioactive than short or tightly bundled CNT/F $[6,7,12,23,52,54,93,96,116-120]$. Specific studies on CNT/F diameter, with consistent length, have not been as extensively investigated as a determinant from toxicity outcomes of CNT/F exposure. These studies, sometimes as a comparison of MWCNT to SWCNT, found that increasing diameter can be associated with less toxicity than thinner fibers in terms of inflammation, histopathology changes, alveolar fibrosis, disrupting membrane integrity, and genotoxicity, while other studies link greater diameter to enhanced macrophage interactions, as well as greater apoptosis and inflammation [9-14, 87, 100, 121]. 


\subsubsection{Nominal tube diameter}

As previously noted, company-provided diameter was the initial segregator for deciding which CNT/F to evaluate for toxicity to ensure broad representation of particle sizes. Preliminary evaluations by electron microscopy of the samples also suggested that length was likely to vary with diameter, thereby creating a good representation of the CNT/F class of materials produced and used in U.S. facilities.

To confirm the nominal tube diameters $(6-150 \mathrm{~nm})$ reported by the company (Table 1$)$, the CNT/F materials were dispersed in isopropanol and analyzed using scanning transmission electron microscopy (STEM). Two hundred individual tubes for each material were measured and the following parameters were determined: geometric mean, arithmetic mean, range, and median of diameters (Table 1). The samples were further characterized by binning into specific diameter ranges (Figure 3). From STEM, MW \#1-4 had geometric means ranging from 12-20 nm (arithmetic means of 13-26 nm) (Table 1). These values were similar to the range of company reported diameters of 6-30 $\mathrm{nm}$. There was a range of 6-275 $\mathrm{nm}$ in diameters but very few tubes of MW \#1-4 had nominal tube diameters above $50 \mathrm{~nm}$. The geometric mean diameter of the benchmark material, MW \#5, was found to be $63 \pm 1 \mathrm{~nm}$ (arithmetic mean of $67 \pm 2 \mathrm{~nm}$ ) with a range of 21-168 nm, slightly larger than previous reports of a mean of $49 \mathrm{~nm}$ [51] but in agreement with other studies [38, 40, 43, 94]. MW \#6-7 were larger in diameter than MW \#1-4 but, on average, smaller than MW \#5 (Table 1). Interestingly, while the mean suggests materials smaller in diameter than MW \#5, the range and distribution of particles was greatest in the larger size bins (> $150 \mathrm{~nm}$ ) for MW \#67 compared to all other MWCNT (Figure 3). CNF \#1 and 2 had diameter geometric means that were similar to each other at $102 \pm 1 \mathrm{~nm}$ (arithmetic mean $110 \pm 3 \mathrm{~nm}$ ) and $103 \pm 1 \mathrm{~nm}$ (arithmetic mean $110 \pm 3$ $\mathrm{nm})$, respectively (Table 1).

It was clear that the central tendency to only include the mean of the distribution of particle dimensions, especially when evaluating MW \#6 and \#7, did not have enough resolution to fully characterize and distinguish one material from another, a critical factor to understand and model toxicity 
outcomes based on material properties. The heterogeneity in diameter size distributions of the CNT/F was assessed from the histograms in Figure 3. The 50\% accumulation or cut-off point was determined by curve fitting using the sigmoidal function and is represented by the dashed line with the nominal size value represented by the upper $\mathrm{x}$-axis. The point of 50\% accumulation was rapidly achieved for MW \#1-4 within the first two bins indicating most particles were less than $25 \mathrm{~nm}$ in diameter. Beginning with MW \#5, a right shift can be seen, reflecting an increase in diameter. While MW \#5 had a significantly larger population of tubes around $64 \mathrm{~nm}$ in diameter, the distribution had a smaller range of particle widths; virtually all particles were contained in three bins, compared to MW \#6 and 7. While not large in absolute number, subpopulations of larger diameter tubes were found in MW \#6 and 7 that were not observed for other MWCNT. CNF \#1 and 2 had a similar profile and distribution. Compared to the MWCNT, the shift in $50 \%$ accumulation towards larger size bins was more distinguished for the CNF and provided a clear distinction from MW \#1-4.

\subsubsection{Nominal tube length}

Lengths were not reported by all companies, and those reported had a range of 0.1-200 $\mu \mathrm{m}$ (Table 1). As with diameter, the nominal tube length was determined on tubes/fibers in parallel with diameter to create paired STEM measurements. Two hundred individual tubes or fibers for each material were measured. The summary of length measurements was presented as arithmetic mean, geometric mean, range, median, and binning by specific diameter ranges (Table 1, Figure 4). MW \#1-4 had geometric mean lengths ranging from $0.67-1.41 \mu \mathrm{m}$ (arithmetic means of $0.80-1.84 \mu \mathrm{m}$ ) (Table 1 ). MW \#1 was the shortest by average length followed by MW \#3, with virtually all length values being segregated in the initial bin (0-2 $\mu \mathrm{m}$ ) (Figure 4). MW \#5 measured much longer than MW \#1-4 at 4.39 $\pm 2.07 \mu \mathrm{m}$ (arithmetic mean of $5.62 \pm 0.29 \mu \mathrm{m}$ ) with a range of 1.2-25.8 $\mu \mathrm{m}$. The measured length is consistent with previous reports of MW\#5/MWCNT-7 [51]. On average, MW \#6-7 were shorter than MW \#5 but longer than MW 
\#1-4 (Table 1). The distribution of longer nominal tubes for MW \#5-7 was greater than MW \#1-4. CNF \#1 measured 3.64 $\pm 2.36 \mu \mathrm{m}$ (arithmetic mean of $5.23 \pm 0.36 \mu \mathrm{m}$ ) in length and CNF \#2 was $2.16 \pm 2.31 \mu \mathrm{m}$ (arithmetic mean of $3.20 \pm 0.28 \mu \mathrm{m}$ ). The length differences between CNF \#1 and 2 was notable as CNF \#2 was $40 \%$ shorter on average with virtually identical diameters. The arithmetic means of MW \#5, \#7

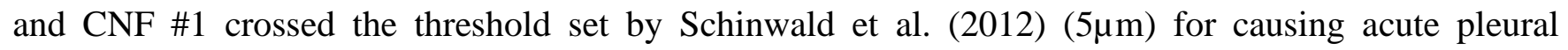
inflammation [110].

Nominal tube lengths were binned and depicted in histograms found in Figure 4 with the cut-off points at 50\% accumulation indicated in each case. For MW \#1-4, almost all (96\%) nominal tube lengths were concentrated in the first two size bins. The cumulative distribution of particles and the $50 \%$ length accumulation cut-off were shifted to the right for MW \#5-7 and CNF \#1-2. Overall, bulk samples containing tubes of greater nominal length were more common in MW \#5-7, and CNF \#1-2, with notably greater length particle populations in MW \#5, 7, and CNF \#1.

\subsubsection{Aspect ratio}

Aspect ratio was a critical measurement considered in the original fiber paradigm. In the 1970s and 1980s, Stanton published his early work linking high aspect ratio materials, particularly glass fibers and asbestos with increased toxicities including lung cancer incidences and mortality [111, 112]. A reanalysis of the research completed in 1980 by Bertrand and Pezerat used multiple regression analysis to conclude that the carcinogenicity of fibers was a continuous spectrum that must include both length and diameter, as a greater aspect ratio can be indicative of greater carcinogenicity [103]. While aspect ratio is an inherent description of length to diameter, the values for each material, including the distribution, were considered for toxicity outcomes.

Individual tube aspect ratio was quantified from STEM measurements as the diameter and length measurements were paired. These measurements were as follows (Geometric Mean \pm Geometric standard 
deviation, GM \pm GSD): $53 \pm 2,96 \pm 2,50 \pm 2,76 \pm 2,69 \pm 2,73 \pm 2,78 \pm 2,36 \pm 2$ and $21 \pm 2$ for MW \#1-7 and CNF \#1-2, respectively. MW \#4-7 had a slightly higher aspect ratio compared to MW \#1 and \#3. CNF \#1 had a lower aspect ratio compared to all $\mathrm{MW}$ due to the notably larger diameter, which was even less for CNF \#2 given a similar diameter, but shorter length compared to CNF \#1. As with length and diameter, aspect ratios were binned and histograms with corresponding accumulation curves were generated and can be found in Figure 5. All materials had a wide distribution of aspect ratios. There was a trend for the peak aspect ratio to be from 50-100 for all materials except CNF \#2. The CNF had a greater leftward distribution with CNF \#2 having a significant population of fibers with an aspect ratio of approximately 20 . Due to the differences in length, CNF \#1 and 2 had notably different distributions of aspect ratios.

\subsubsection{Two-dimensional sizing of agglomerates}

Previous studies have considered the role of CNT agglomeration as a determinant of toxicity outcome, particularly within the context of genotoxicity, macrophage recognition, the activation of downstream inflammatory cascades, and pulmonary fibrosis [15, 56, 64, 122-124]. Rod-like and less tangled particles, including singlets, were more likely to influence inflammation histopathology outcomes by inducing more pulmonary fibrosis, and impact extrapulmonary translocation [10, 56, 122]. Furthermore, agglomeration patterns and size are relevant factors in human occupational exposures and respirability $[68,69]$.

Using SEM images, size measurements of particle agglomerates were completed following dispersion in our physiologic dosing medium, commonly referred to as dispersion medium (DM). We have previously described how the sample preparation mimics collected personal breathing zone samples of workers [19]. Representative SEM images can be found in Figure 2. Based on the dimensions, particles in this study were categorized into two distinct groups, spherical or bundled agglomerates. Spherical agglomerates were defined as tangles of tubes/fibers that had an aspect ratio of less than 3:1; this 
convention was adapted from earlier workplace exposure assessment studies [68]. The second category of agglomerates was referred to as "bundles." These bundled agglomerates were tangles of particle with an aspect ratio greater than 3:1. These bundles varied in the number of tubes/fibers, with some having only a couple. In sizing these structures, the major and minor axes of the bundles were reported as length and diameter, respectively.

MW \#1 and \#3 were similar with spherical agglomerates composing the bulk (87\% and 83\%, respectively). A second subpopulation of bundled agglomerates was present, but not dominant. Spherical agglomerates of MW \#1 averaged $0.96 \pm 2.01 \mu \mathrm{m}$ (arithmetic mean of $1.28 \pm 0.16 \mu \mathrm{m}$ ) in diameter and MW \#3 has spherical agglomerates averaging $0.66 \pm 1.84 \mu \mathrm{m}$ (arithmetic mean of $0.81 \pm 0.10 \mu \mathrm{m}$ ) in diameter. Bundled agglomerates of MW \#1 and \#3, which represented less than $20 \%$ of the total sample exceeding a 3:1 dimension, had lengths under $2 \mu \mathrm{m}$ and diameters under $0.4 \mu \mathrm{m}$ on average.

MW \#2 was a unique material that formed quite large agglomerates that were not spherical, but rather interconnected 'rivers' of highly entangled cross-linked MWCNT (Figure 2). These agglomerates were "bundled" agglomerates with a high aspect ratio and two subpopulations were distinguished (Table 2). The large bundled agglomerates averaged $49.55 \pm 3.58 \mu \mathrm{m}$ in length and had an average diameter just under $10 \mu \mathrm{m}$. The second subpopulation was found as more loosely bundled, smaller structures, likely agglomerates of singlet tubes as compared to the entangled cross-linked MWCNT. This subpopulation had arithmetic averages of $3.80 \pm 0.38 \mu \mathrm{m}$ in length and $30 \mathrm{~nm}$ in diameter. The two populations also highlight that the singlet nominal tube diameter and length of MW \#2 was not entirely representative of the material as it did not account for the large bundled agglomerates.

Beginning with MW \#4, there was a rather dramatic transition from spherical agglomerates to bundled agglomerates (Table 2). In fact, less than 0-4 \% of any of MW \#4-7 or CNF \#1-2 were categorized as spherical agglomerates using our criteria. As the materials increase in diameter, the materials become almost exclusively small bundles and singlets that assume a more classic fiber-like appearance (Figure 2). 
The bundled agglomerates, more representative of a fiber-like appearance, were representative of the physical dimensions. Specifically, the length of the bundled agglomerates for MW \#4 and CNF \#2 were on average 50-75 \% shorter compared to MW \# 5-7 and CNF \#1.

\subsubsection{Hydrodynamic diameter and zeta potential}

The hydrodynamic diameter, which qualitatively reflects the agglomerated state of the CNT in aqueous solution, was evaluated using dynamic light scattering (DLS). The hydrodynamic diameter ranged between 478 - $771 \mathrm{~nm}$ (Table 2). MW \#4 was found to have the smallest hydrodynamic diameter, followed by MW \#5. Similar values were found for MW \#1, 3, 7, and CNF \#1 and 2, and MW \#2 and MW \#6 had the largest hydrodynamic diameters.

Zeta potential, the electrokinetic potential at the interface of the particle surface and aqueous solution, was evaluated by measuring the electrophoretic mobility of the particles in solution by phase analysis light scattering. Zeta potential of a nanomaterial is indicative of its stability in a solution. Minimal differences in zeta potential were observed between these materials (Table 2).

\subsubsection{Surface area}

Surface area has been a central measurement for ultrafine particle characterization [125]. Studies have focused on how surface area was a primary determinant of toxicity, especially with metal oxides [126-129]. While surface area is inversely related to nominal tube diameter and decreases with agglomeration, the quantification of the surface area of CNT/F can pose some limitations due to their physical structure $[22,130,131]$. For example, the interior space of variable concentric layers paired with porosity, grooves, and other surface topography can lead to variation in measurements of surface area. A few studies have linked increased surface area of CNT/F to more pronounced toxicity outcomes, including genotoxicity and inflammation $[12,122,132]$. In this study, all CNT/F were analyzed using the same 
methodology, allowing for adequate comparisons between materials (Table 3). The surface areas follow the expected relationship that smaller diameter corresponded to greater surface area on a mass-to-mass basis. MW \#1-3 had the greatest surface area. MW \#4 was intermediate indicating a transition point in physical dimensions. MW \#5-7 and CNF \#1 and 2 have the smallest surface area, almost an order of magnitude less than MW \#1-3.

\subsubsection{Dustiness}

Particle dustiness is a quantification of the tendency of a dry powder to aerosolize, an important aspect for understanding the potential for human occupational exposure. Two independent measurements, total and respirable dustiness, were simultaneously determined as previously described [20] and well suited to characterizing these CNT/F materials. Total dustiness was the percent of the total dust (sample using a closed face cassette) that can be aerosolized from the test sample, while the respirable fraction (sampled with a cyclone) was the percent of the aerosolized dust that can penetrate to the deep airways, or the alveolar region. Total dustiness may be approximated to the inhalable dustiness fraction, particularly with these CNT/F materials [133] MW \#1, \#2, \#5, and CNF \#1 had total dustiness that ranged from 3-14 $\%$ and a respirable dustiness that ranged from $0.8-2.4 \%$. CNF \#2 was not measured but was expected to be very close to the values of CNF \#1 and MW \#5 published previously [20]. The total dustiness of MW \#3, \#4, \#6, and \#7 ranged from 0.2-0.5\%, approximately an order of magnitude less that the other

materials. The respirable dustiness ranged from $0.08-0.20 \%$. The results indicate greater dustiness for some CNT/F compared to others but not a consistent pattern with relationship to physical dimensions or surface area. 


\subsubsection{Density}

As CNT/F mostly occur as agglomerates, the aerodynamic behavior is determined by the effective density of the agglomerates [15]. Most CNT/F exposures are performed on a mass basis and the NIOSH REL is based on mass concentration of elemental carbon. Given that density is directly proportional to mass, theoretically, the lower the effective density, the more CNT/F particle would be needed for equivalent dosing by mass. Recent computational modeling of engineered nanomaterials included density in the analyses $[80,134,135]$ with some indication it was a primary driver of toxicity [134]. Measurements of bulk and tapped skeletal density were performed for all CNT/F (Table 3). MW \#1, \#3, and \#4 were comparatively denser than MW \#2 and MW \#5 by an order of magnitude. The remaining materials, MW \#6, MW \#7, CNF \#1, and CNF \#2 were intermediate from the above-mentioned materials. As concluded with dustiness, there was no apparent consistent pattern that linked skeletal density to other physical dimensions, surface area, or dustiness.

\subsubsection{Chemical and metal impurities}

Chemical and metal impurities from the catalysts and production process were usually present at some level in CNT/F end products. Some common metal impurities found in the CNT/F include iron, nickel, chromium, cobalt, copper, zinc, molybdenum, and aluminum. Some of these metals such as iron [26], nickel [136], molybdenum [137], chromium and cobalt [101] were found to influence the toxicological profile of CNT. Thirty-one metals and chemical impurities were screened using inductively coupled plasma atomic emission spectroscopy (ICP-AES). Most of the thirty-one metals evaluated were below their respective analytical limits of detection (LODs). The metals that were present in one or more CNT/F include iron (0.27-6.2\%) and aluminum (0-2.2\%) (Table 3). Trace amounts of cobalt, molybdenum, zinc, nickel, manganese, lead and cadmium were in range of $(0-0.17 \%),(0-0.05 \%),(0-$ $0.1 \%),(0-0.004 \%),(0-0.006 \%),(0-0.002 \%)$ and $(0-0.0005 \%)$ respectively. Most of the CNT/F currently 
used in U.S. facilities had minimum trace amounts of metal residues. Iron was a consistent catalyst ranging from $0.27 \%$ to $6.17 \%$ (Table 3). MW \#6 and \#7 had the highest levels of residual iron catalyst. Another metal of note was aluminum which was present in MW \#3 at $2.1 \%$ with residual amounts of $0.31 \%$ or less in other CNT/F (Table 3). All other metals were at levels of $0.17 \%$ or less.

\subsubsection{Thermal stability, degradation, and purity}

Thermal stability, degradation, and purity of CNT/F was assessed using thermogravimetric analysis (TGA). This technique analyzes change in the weight of a specimen in relation to increasing temperature. The oxidation onset temperature, the temperature at which the oxidation of CNT/F starts, is considered a measure of thermal stability and degradation varied across the CNT/F. The onset temperature for the $\mathrm{CNT} / \mathrm{F}$ ranged from $550-735^{\circ} \mathrm{C}$ (Table 3). The residual ash, or the content left after complete oxidation, was evaluated to determine the purity of the CNT/F. The percentage of residual ash for MW $\# 1-7$ and CNF 1-2 was $1.74 \pm 0.01 \%$ (means \pm SD), $3.98 \pm 0.26 \%, 8.21 \pm 0.26 \%, 4.75 \pm 0.07 \%, 1.11 \pm 0.28 \%$, $7.88 \pm 0.15 \%, 8.95 \pm 0.29 \%, 1.79 \pm 0.12 \%$, and $2.21 \pm 0.16 \%$ respectively (Table 3 ). These values are primarily indicative of metal content and were generally consistent with relative levels of residual metal catalysts determined using ICP-AES. MW \#3, \#6, and \#7 had the greatest residual ash and results are consistent with the higher amount of metal catalyst measured.

\subsubsection{Polycyclic Aromatic Hydrocarbons and Endotoxin}

Airborne background contaminants and byproducts like polycyclic aromatic hydrocarbons (PAHs) and endotoxin, a component of the bacterial cell wall, can be a major influence on the toxicity profile of various engineered nanomaterial and environmental particulates [138-140]. Previous exposure and emission monitoring at a CNF production facilities indicated the presence of PAHs with an average concentration up to $336 \mu \mathrm{g} / \mathrm{m}^{3}$ [141]. To rule out the influence of PAHs and endotoxin, gas 
chromatography-mass spectrometry with selected ion monitoring (GC-MS SIM) and limulus amebocyte lysate assay were performed, respectively. The levels of PAHs and endotoxin in the CNT/F were below their LODs. The lack of endotoxin was supported by no significant induction of tumor necrosis factor- $\alpha$ production (described below) from epithelial cells at the highest CNT/F dose tested.

\subsubsection{Acellular Reactivity}

Multiple physicochemical characteristics of the CNT/F including residual metal catalysts, surface defects, functionalization, and redox active organic matter, such as quinones, will alter the reactivity of the nanomaterial in biological matrices. This can lead to an imbalance in redox homeostasis that can trigger oxidative stress and toxicity. The ferric reducing ability of serum (FRAS) assay was used as an acellular screen to determine the antioxidant capacity, or the ability of CNT/F to react in biological matrices and deplete antioxidants. This assay serves as a screen for oxidative stress and potential toxicity [142]. Compared to untreated serum, reaction with CNT/F reduced the antioxidative capacity of serum by 65-100\% (Table 3). CNF \#1 and 2 had $100 \%$ remaining antioxidative capacity, indicating that these materials were the lowest in their ability to independently react and induce oxidative stress. MW \#1 and MW \#7, two very distinct CNT materials in terms of physical dimensions, consumed the most serum antioxidants as indicated by the lowest remaining \% antioxidative capacity. The remaining CNT ranged from $75-91 \%$.

\subsubsection{Grouping CNT/F by Principal Component Analysis of Physicochemical Characteristics}

As a first step, feature selection using the Boruta algorithm was performed on three sets of physicochemical property data for the nine different CNT/F materials: 1) detailed characterization of length (L), diameter/width (labeled as W for figure clarity for easier distinction from $\mathrm{L}$ ), and aspect ratio (AR) from the binned data from Figures 3-5 (Figure 6B; L-W-AR binning); 2) standard physicochemical 
data using means only from Tables 1-3 (Figure 6C; Means only); and 3) the combination of L-W-AR and means only data (Figure 6A; All characterization). Figure 6 displays the principal components analysis (PCA) results for different CNT/F samples with confirmed variables of importance from the three separate analyses (Supplemental Figure S1 A-C). It should be noted that the PCA plots did not change without feature selection (Supplemental Figure S2). The first three principal components describe $71 \%, 68 \%$ and $82 \%$ of the total variability among materials for the 'all characterization', 'L-W-AR binning', and 'means only' parameters, respectively. Most importantly, the PCA analysis of L-W-AR and all characterization variables suggested a segregation of MW \#1-4 materials from MW \#5-7 and CNF \#1-2 (Figure 6A-B). Overall, a combination of larger lengths and widths separated one group of materials (MW \#5-7, CNF \#1-2) from the second group of materials (MW \#1-4) (Figure 6A-B; Supplemental Figure S1 A-C). The categorization of MW \#5-7 and CNF \#1-2 together in the same group indicates common physicochemical characteristics of these materials. However, this was not the case with PCA using traditional variable data which were based on mean values only (Figure 6C). Often, the literature reports only mean values without including the detailed size distributions for physical dimensions. Previous studies proposed that providing distributions of dimensional characteristics would better segregate different CNT/F for grouping and toxicity [101]. The difference in the material segregation between means only compared to L-W-AR binning and all characterization suggests that varying the input parameters will influence conclusions drawn in terms of which physicochemical characteristics may drive specific toxicity outcomes. The variance in the PCA plots provided two initial suggestions: 1) binning of the physical dimensions may be critical for accurate representation of the materials and potential toxicity and 2) binning of the physical dimensions without significant additional physicochemical characterization may alone be enough to group CNT/F. The latter point agrees with the lack of a consistent pattern when comparing surface area, density, residual metal catalyst, dustiness, etc. for the various CNT/F. 
Another important finding was that the use of L-W-AR binning alone parameters further separated CNF \#1 from CNF \#2 and grouped CNF \#1 together with MW \#5-7 (Figure 6B). Furthermore, a closeclustering of CNF \#1 with MW \#6 and their overall grouping with MW \#5 and \#7 along with correlated L-W-AR binning variables in the PC1 dimension, supports the notion that a greater range of sizes can be found in $\mathrm{CNF} \# 1$ compared to CNF \#2. The PC1 dimension correlates MW \#6 and CNF \#1 materials with L15, L10, Lmore, W0.1, W0.2 and W0.3 variables. Similarly, a correlation of MW \#5 and MW \#7 with the variables L6, L8, L10, L25, L30, W0.15 and Wmore was also observed. Overall, these results suggest that larger lengths and diameters separate MW \#5-7 and CNF \#1 from the rest of the materials investigated. Importantly, the separation does not indicate a large fraction of the CNT/F sample has those larger dimensions (Figure 3-4) but rather the sample contains some proportion of tubes with those specific nominal physical dimensions.

\subsubsection{In vitro Toxicity Assessment}

\subsubsection{Cell Viability}

Human bronchiolar epithelial cells (BEAS-2B; selection detailed in Methods) were challenged with the nine $\mathrm{CNT} / \mathrm{F}$ at $0.024,0.24,2.4$, and $24 \mu \mathrm{g} / \mathrm{ml}$ for $24 \mathrm{~h}$ and cell viability was assessed by measuring the reduction of cell proliferation reagent WST-1 (Figure 7A). Dose selection and relevance is detailed in the Methods. The lowest two doses $(0.024$ and $0.24 \mu \mathrm{g} / \mathrm{ml})$ caused no significant change in cell viability. The highest dose $(24 \mu \mathrm{g} / \mathrm{ml})$ significantly reduced viability with all the materials tested except with MW \#2. CNF \#2 induced $\sim 45 \%$ reduction in cell viability. The $2.4 \mu \mathrm{g} / \mathrm{ml}$ dose induced a small but significant reduction in cell viability for MW \#1-3 and CNF \#1. These toxicity results are consistent with previous results $[46,47,143]$. The $\mathrm{IC}_{80}$ for MW \#1-7 and CNF \#1-2 ranged from 11-43 $\mu \mathrm{g} / \mathrm{ml}$. Subsequent studies of genotoxicity were done at 0.024 and $2.4 \mu \mathrm{g} / \mathrm{ml}$ in accordance with OECD TG487 [144] and ICH S2(R1) [145] guidance for $80 \%$ or greater cell viability. 


\subsubsection{Oxidative stress}

Reactive oxygen species (ROS) consisting of hydrogen peroxide, singlet oxygen, superoxide anion, hydroxyl radical, and hypochlorous acid are constantly regulated by the cells, which is essential to maintain homeostasis. Epidemiology studies of workers exposed to CNT/F during their manufacturing or use in downstream applications found alterations in oxidative stress markers and antioxidant enzymes [70, $71,78,137]$. Animal and in vitro studies using various cell types, including epithelial cells, confirmed induction of oxidative stress with various CNT/F exposures. The response was amplified by metal impurities and was found to be dependent on the physicochemical characteristics that influence the reactivity, cellular internalization, and biopersistence [116, 146, 147]. In order to assess the oxidative stress potential of the nine CNT/F, BEAS-2B cells were exposed for $24 \mathrm{~h}$ at concentrations of $0-24 \mu \mathrm{g} / \mathrm{ml}$ and then labeled with CellROX, a non-fluorescent cell-permeant dye that fluoresces upon oxidation by ROS. Fluorescence per cell was evaluated by flow cytometry. Only the highest dose $(24 \mu \mathrm{g} / \mathrm{ml})$ induced a significant oxidative stress response for MW \#4-7, and CNF \#1-2 (Figure 7B). There was a trend for an effect in MW \#6-7 and CNF \#1-2 at $2.4 \mu \mathrm{g} / \mathrm{ml}$. The CNT/F with smaller physical dimensions (MW \#1-3) did not induce ROS even at the highest concentration tested.

\subsubsection{Cytokines, chemokines, and growth factors}

A selection of 27 cytokines, chemokines, and growth factors were assessed from cell supernatant following exposure to 2.4 and $24 \mu \mathrm{g} / \mathrm{ml}$ of each of the nine CNT/F for $24 \mathrm{~h}$ (Figure 7C). Many of the measured proteins were altered for most of the $\mathrm{CNT} / \mathrm{F}$ tested. MW \#2 exposure altered the least number

of proteins. The reduced response was likely due to the large bundled aggregate fraction (Table 2) not having the same cellular effect as the other CNT/F. MW \#6 and \#7 caused the most significant changes, especially at the lower dose evaluated, indicating these materials may be more adept at altering cellular signaling than other materials in this study. All materials except MW \#2 induced a significant increase in 
primary modulators of innate inflammation, IL-6, IL-8, IL-1 $\beta$, etc., at the high dose and several at the low dose (e.g., MW \#6-7). Some molecules assessed, including IL-10, an anti-inflammatory cytokine, were significantly reduced. FGF was increased while other growth factors measured, VEGF and PDGF- $\beta \beta$, were generally decreased. At the higher dose, suppression of certain cytokines was more evident with MW \#5-7 and CNF \#1-2.

\subsubsection{Genotoxicity}

The potential for CNT/F to cause carcinogenicity is an area of active research $[148,149]$. In vivo and significant in vitro evidence suggested adverse health consequences following inhalation to CNT/F. One material, MWCNT-7/Mistui-7, has been shown to be a complete carcinogen in rodent models, which led IARC to designate this material as possibly carcinogenic to humans (Group 2B) [44]. All other materials were considered as Group 3 as there was insufficient evidence to classify otherwise [115]. The 2020-2024 Report of the Advisory Group to Recommended Priorities for the IARC Monographs indicates MWCNT as a high priority and ready for evaluation within five years [37]. While human health effects studies have begun globally, the latency for carcinogenicity has not been reached [70-73, 77-79, 89, 90].

To date, most studies concerning the potential carcinogenicity of CNT/F have used in vitro approaches to evaluate genotoxicity. The approach allows for a rapid screening after which detailed mechanistic and in vivo studies can be conducted to expand initial evidence of genotoxicity. The micronucleus assay was used to determine if $\mathrm{CNT} / \mathrm{F}$ treatment results in disruption of the mitotic spindle or chromosome breakage. This approach also allows for the simultaneous evaluation of a large group of materials. Parallel cultures of human epithelial BEAS-2B cells were exposed to 0.024 and $2.4 \mu \mathrm{g} / \mathrm{ml}$ of the $9 \mathrm{CNT/F}$, with MW\#5 (Mitsui-7/MWCNT-7) serving as a documented positive control, for $24 \mathrm{~h}$ and the number of cells with micronuclei were quantified (Figure 8A-B). The screening approach, including cell type and exposure concentration, has been used previously by our group [42, 47, 143]. Viability in 
the high dose was $\geq 80 \%$, and the low dose had $\geq 97 \%$ viability. In DM-exposed cells, few micronuclei were detected, and background incidence was similar to previous studies $[42,47]$. All CNT/F materials at both the low and high dose induced significant increases in micronuclei number except for the low dose of MW \#2 (Figure 8B). The treatments were not significantly different from one another.

In complement, the phosphorylation of $\mathrm{H} 2 \mathrm{AX}$, a cellular response to repair double-strand DNA breaks, was evaluated. Flow cytometry was used to quantify phosphorylated H2AX, or $\gamma-\mathrm{H} 2 \mathrm{AX}$. All high dose-treated cells induced $\gamma$-H2AX except for MW \#2 (Figure 8C). Increased levels of $\gamma$-H2AX were also measured for MW \#7, CNF \#1 and CNF \#2 for the low dose treatment. For CNF, the low and high doses had similar effects. While measurements of $\gamma-\mathrm{H} 2 \mathrm{AX}$ was considered a low priority indicator of genotoxicity as it does not directly indicate irreversible mutations [149], the response was similar to the micronuclei outcome.

\subsubsection{Hierarchical clustering and PCA of the cellular outcomes}

A hierarchical clustering analysis (HCA) was performed to distinguish or discriminate the BEAS2B cellular outcomes induced by $\mathrm{CNT} / \mathrm{F}$ materials with varying physicochemical characteristics. HCA, unlike model-dependent analyses such as supervised machine learning methods, is a model-free statistical approach that makes no a priori assumptions about the class identification of data. The resulting dendrogram from the HCA analysis of physicochemical properties of 'all characteristics' combined with outcomes of the four primary in vitro assays, cell viability, cellular oxidative stress, micronuclei formation, and $\gamma-\mathrm{H} 2 \mathrm{AX}$, is depicted in Figure 9A. Overall, the dendrogram initially divided CNT/F exposure responses into two clusters or groups, one predominantly containing MW \#1-4 together with the control group, and the other containing MW \#5-7 and CNF \#1-2 (Figure 9A). HCA was also done for outcomes in comparison to the 'L-W-AR binning' and 'means only' characterization profiles. The L-WAR binning profile produced the same two clusters (Figure 9B) as developed using all characterization 
parameters (Figure 9A). The means only HCA shifted MW \#4 into the cluster with MW \#5-7 and CNF \#1-2, suggesting similarities resembling more MW \#6-7 than MW \#1-3 (Figure 9C), indicating the input selection of characteristics can vary the grouping in relation to toxicity outcomes. PCA results from 'all characterization' combined with the four primary in vitro assay outcomes (Supplemental Figure 3A) grouped similarly to 'L-W-AR binning' (Supplemental Figure 3B), producing a separation between the two clusters. The 'means only' with in vitro outcomes (Supplemental Figure 3C), like the HCA dendrogram (Figure 9C), was less clear in distinguishing groups of CNT/F.

HCA analysis was done for 'all characteristics' and the four primary in vitro outcomes along with inflammatory protein production (Supplemental Figure 4). The grouping was unaltered compared to Figure 9A except for a clearer separation in the two sub-clusters. This was evident as MW \#6-7 had a significant grouping of induced inflammatory proteins compared to MW \#5 and CNF \#1-2. What became evident from Figure 9A-B and Supplemental Figure 4 was that binning of the aspect ratio data did not segregate to any particular outcome and may be unnecessary for the HCA. To illustrate, Supplemental Figure 5, HCA without aspect ratio binning, created the same two clusters of MW \#1-4 and MW \#5-7 / CNF \#1-2 for 'all characterization' (Supplemental Figure 5A) and 'L-W-AR binning' (Supplemental Figure 5B) when considering the four primary in vitro epithelial toxicity outcomes. Within each subcluster, co-clustering between materials was also evident as all variations in HCA pulled out the two CNF from the CNT and MW \#6 and \#7 clustered together even without secreted protein changes as in Supplemental Figure 4. There were a few subtle differences in pairings between MW \#1-4 and control for the L-W-AR binning HCA compared to all characterization. As an additional step, HCA analysis was done, without aspect ratio, to include the altered protein changes with the four primary toxicity outcomes and the three variations in characterization parameters. Interestingly, all three scenarios (Supplemental Figure 6A-C) now had the same two clusters, meaning the 'means only' HCA placed MW \#4 with MW \#1-3 instead of MW \#5-7 and CNF \#1-2. Previously for 'mean only' HCA (Figure 9C), MW \#4 was 
combined with MW \#6-7. It was clear from Supplemental Figure 6C that the large group of induced inflammatory proteins for MW \#6-7, not seen with MW \#4, altered the clustering. This series of analyses suggests that when using 'means only' for physicochemical characterization, additional toxicity data may be necessary to accurately categorize all materials in terms of epithelial cell toxicity. It also indicated that altered inflammatory protein concentrations, at least the panel used in this study, were not necessary to group $\mathrm{CNT} / \mathrm{F}$ in terms of epithelial toxicity if binning of physical dimensions was available.

We next considered just the four primary outcomes of in vitro toxicity and protein production with no physicochemical characteristics. The HCA analysis also grouped MW \#1-4 separately from MW \#5-7 and CNF \#1-2 (Supplemental Figure 7). The toxicity only grouping consistently matched HCA analyses using 'all characterization' or more simply the 'L-W-AR binning' as compared to the 'means only' characterization from Figure 9. The separation of oxidative stress, micronuclei formation, and $\gamma-\mathrm{H} 2 \mathrm{AX}$ from protein production when considering outcomes only (Supplemental Figure 7) further supports the consistency of grouping when physical dimension binning was determined and analyzed without inflammatory protein production (Supplemental Figure 4 and 6). The separation also suggests that epithelial cell viability and inflammatory cytokine production, as assessed by the panel used, were not primary drivers of genotoxicity.

The various analyses allowed for interpretation of which physicochemical properties drive which epithelial cell toxicity outcomes. Three of the four in vitro outcomes evaluated, oxidative stress, micronuclei formation, and $\gamma-\mathrm{H} 2 \mathrm{AX}$, grouped with certain physicochemical properties that were identified in Figure 9 and Supplemental Figures 4-6 as distinguishing between the two CNT/F clusters. Bins of larger lengths and diameters (W), including L15, L20, L25, L30, W0.1, W0.2, and Wmore, clustered with the outcomes. Also clustering with the toxicity outcomes were bundled agglomerate singlet percentage, length, and diameter from the two-dimensional sizing (Table 2). Inherently, that would be expected as the increasing physical dimensions of length and diameter transition the CNT/F from a spherical agglomerate 
(e.g., MW \#1 and \#3) to a more elongated bundled agglomerate. Depending on the parameters for HCA, the fourth primary outcome, cell viability, sometimes grouped with the other three toxicity variables of importance (Figure 9B; Supplemental Figure 4), but other times did not (Figure 9A; Supplemental Figure 5 and 7), suggesting that cell viability may not always be a useful assay for determining differential toxicity among materials. Overall, MW \#1-4 materials clustered separately from MW \#5-7 / CNF \#1-2. While all materials induced significant micronuclei formation (Figure 8B), when combined with $\gamma-\mathrm{H} 2 \mathrm{AX}$ (Figure 8C) and cellular oxidative stress (Figure 7B), there was a propensity for greater severity in the cluster of materials that contained a greater proportion of tubes/fibers with larger physical dimensions, MW \#5-7 / CNF \#1-2 (Figure 9). This was further supported by the fact that control samples always clustered with MW \#1-4.

Of note was the close clustering of cellular oxidative stress with $\gamma$-H2AX levels and micronuclei formulation. This was especially evident with physicochemical variables of larger lengths and widths and bundled agglomerate characteristics. The finding was consistent of genotoxicity through indirect oxidative stress-related mechanisms upon exposure to high aspect ratio nanomaterials [150, 151]. Increased oxidative stress, as a result of lysosomal damage by inefficient phagocytosis of high aspect ratio nanomaterials, can cause double-stranded DNA damage and chromosomal aberrations leading to micronuclei formation. $\gamma-\mathrm{H} 2 \mathrm{AX}$, an early indicator of DNA-double strand breakage and a process that precedes the formation of micronuclei, segregated mostly with larger nominal tube/fiber diameter and length of CNT/F. Oxidative stress responses clustered together with bundled agglomerate length support the notion that oxidative stress due to inefficient cellular handling of aggregated particles and/or lysosomal damage by particles of larger dimensions could be a contributing mechanism of genotoxicity, especially for MW \#5-7 and CNF \#1-2.

Caution should be taken not to overstate the associations of the larger CNT/F as SWCNT have been shown to be potent inducers of chromosomal damage $[42,45,149]$. Also, MW \#1 and \#3 in this study 
induced significant effects complementing a recent pulmonary exposure study of a MWCNT $7.5 \mathrm{~nm}$ in diameter induced cancer [152]. Kuempel et al. concluded in a review of CNT genotoxicity studies that there was not a straightforward relationship between length and genotoxicity, although most of the evaluated studies had CNT length of only a few microns or less [148]. Jackson et al. (2015) and Poulsen et al. (2016) described increased diameter as a physicochemical characteristic linking genotoxicity for the 15 and 10 MWCNT tested in those respective studies $[12,94]$. Those studies represented materials similar to MW \# 1-5. The greater range of CNT/F physical dimensions in this study provided a clearer separation of materials. Overall, all materials, from $\mathrm{MW} \# 1$ to $\mathrm{CNF} \# 2$, had the potential to induce in vitro genotoxicity. When combining cellular oxidative stress and $\gamma-\mathrm{H} 2 \mathrm{AX}$ with micronuclei formation and a broad sampling of the class of CNT/F there was a general shift for greater length and diameter materials to cluster together with some increased severity.

Many of the other physicochemical characteristics did not associate with epithelial cell toxicity outcomes. This does not indicate a lack of importance but more the scope of the endpoints considered. Subsequent studies will evaluate the association of the various physicochemical characteristics with macrophage activation, fibrosis development, and translocation. It was noted that while cellular oxidative stress consistently clustered with micronuclei formation and $\gamma-\mathrm{H} 2 \mathrm{AX}$, there was no clustering with acellular reactivity measured by FRAS or residual metal catalyst. This indicates the residual metal catalyst levels ranging from $0.3-6 \%$ were not the primary drivers of cellular oxidative stress compared with larger physical dimensions. The clustering was confirmed by residual metal catalyst grouping with residual ash measured by TGA as expected (Figure 9; Supplemental Figure 4). In conjunction with anti-oxidative capacity (acellular oxidative stress), aspect ratio, hydrodynamic diameter, and the smaller length bins (L2 and L4) were unable to segregate materials and clustered away from all toxicity outcomes. Additionally, density, specific surface area, zeta potential, the smaller width bins, and spherical agglomerate measurements were also not predictive. Dustiness, while a critical factor for worker exposure assessment, 
was not predictive of epithelial cell toxicity. Cluster analysis of all the induced cytokines together with physicochemical characteristics and toxicity outcomes measured (Supplemental Figure 4) placed the proteins into four groups, two of which were exclusive for proteins. Most proteins did not cluster with the physicochemical characteristics and none with the four primary biological outcomes. While significant changes in inflammatory drivers, growth factors, and cell survival and proliferation signaling molecules occur following $\mathrm{CNT} / \mathrm{F}$ treatment in the epithelial cells, the change in these proteins did not correlate to biological outcomes like oxidative stress and genotoxicity.

\subsubsection{Summary}

Advances in computational analysis are being applied to the almost two decades of engineered nanomaterial research for grouping and understanding the physicochemical drivers of toxicity [80, 134, $135,153,154]$, including studies of carbon nanotubes $[12,101,121,155,156]$. The analyses of the data from this study illustrate that detailed physical dimension characteristics provide a more consistent grouping of $\mathrm{CNT} / \mathrm{F}$ as compared to using only data means. In fact, analysis of binning of nominal tube physical dimensions alone produced a similar grouping as to all characterization parameters. Theoretically, working backwards, a predictive algorithm could be generated that allows classification of CNT/F into distinct toxicity groups based on 200 paired length and diameter measurements. While all materials induced micronuclei formation in human bronchial epithelial cells, when combined with additional parameters associated with genotoxicity, there was an increase in the severity if the sample contained some proportion of materials with larger diameters and longer nominal lengths. The population of nominal tubes with longer length and larger diameters within a sample was not always the majority (e.g., MW \#7), meaning a significant percentage of the tubes with those characteristics was not needed for increased severity of toxicity. The analyses indicate that a more detailed physicochemical characterization of physical dimensions provides better understanding of the differential toxicity within a class of materials, 
implying that evaluating particle characteristic means alone may not be sufficient to accurately segregate CNT/F for certain aspects of toxicity. Subsequent studies analyzing outcomes of inflammation, histopathology, and translocation following $\mathrm{CNT} / \mathrm{F}$ exposure will further develop clustering by physicochemical characteristics and specific endpoint toxicity. In this study evaluating epithelial cell toxicity, all materials induced some level of genotoxicity. However, of the CNT/F evaluated, materials that contained a proportion of tubes with greater lengths and diameters were associated with increased severity.

\subsection{Conclusions}

- Binning of physical dimensions (length and diameter/width) offered greater resolution in terms of grouping $\mathrm{CNT} / \mathrm{F}$ based on physicochemical characteristics compared to using means only. This was further evident when analyzing the physicochemical characteristics and epithelial cell toxicity outcomes.

- Binning of physical dimensions alone offered the same resolution for grouping $\mathrm{CNT} / \mathrm{F}$ as using all physicochemical characteristics suggesting the potential of reduced characterization needed for grouping CNT/F fibers.

- All CNT/F, with the lone exception of the highly aggregated low dose of MW \#2, induced genotoxicity. There was no difference between materials for micronuclei formation.

- When micronuclei formation was combined with cellular oxidative stress and $\gamma-\mathrm{H} 2 \mathrm{AX}$ levels, CNT/F with increasing length and diameter grouped with slightly more toxicity.

- Computational analysis illustrated that increasing length and diameter contribute to greater epithelial cell toxicity. Binning of physical dimensions alone was sufficient to group CNT/F in terms of epithelial cell toxicity. The nature of the bundled agglomerate formation, a reflection of the physical dimensions, also grouped with toxicity outcomes. 
- The increasing length and diameter $\mathrm{CNT} / \mathrm{F}$ do not need to be the majority constituent of the produced material. A small percentage of nominal tubes/fibers with increased length and diameter was sufficient to alter the toxicity profile.

- There was no consistent pattern of density, specific surface area, dustiness, residual metal catalyst, and surface charge associating with physical dimensions or genotoxicity outcomes.

\subsection{Materials and Methods}

\subsubsection{Materials}

All CNT/F used in this study, with the exception of MW \#5 (MWCNT-7/Mitsui-7/), were asproduced materials obtained from six different U. S. primary or secondary manufacturing facilities. Occupational exposure assessments of these facilities were completed to provide insight into human exposure risks and offer direct insight into the vast array of materials utilized [68]. MW \#5 (MWCNT7/Mitsui-7/) was included in this study as a benchmark material as its toxicity profile is well-studied and characterized (Figure 1).

\subsubsection{Characterization}

Length and Diameter: Tube and fiber length and diameter were measured using high resolution scanning transmission electron microscopy as previously described [19]. Briefly, samples of CNT/F were sonicated in isopropanol for 5 minutes. A STEM grid was dipped into the dispersed suspension and used for imaging and analysis. Measuring tools included in the electron microscope's software were used to determine paired length and diameter. Length was determined by connected points at the two extremes without following the curvature of the nanotube or nanofiber. High resolution images were collected with a Hitachi HD-2300 STEM. 
Two-Dimensional Agglomerate Sizing: Samples of CNT/F were prepared in physiologic dispersion medium and the dispersed samples were prepared for field emission scanning electron microscopy (FESEM; Hitachi S-4800, Tokyo, Japan). Measurements were collected using measuring tools of the microscope's provided software (FE-PC SEM Ver. 2.8, Hitachi High Technologies America). The largest crosswise diameter of 75 agglomerates were measured for each material. Materials were subsequently categorized into distinct groups of agglomeration defined as either spherical agglomerates or bundles of fibers with one dimension greater than three times the other dimension, referred to as bundled agglomerates. Bundled agglomerates had both a diameter and length measurement.

Aspect Ratio: Aspect ratio was calculated as the ratio of $\mathrm{CNT} / \mathrm{F}$ length to diameter.

Hydrodynamic Diameter: The hydrodynamic agglomerated size of the various CNT/F dispersed in DM was evaluated using DLS. DLS was performed on a Malvern Zetasizer Nano ZS90 (Worcestershire, UK) equipped with a $633 \mathrm{~nm}$ laser at a $90^{\circ}$ scattering angle. The DLS measurements were performed by dispersing the CNT/F material in dispersion media. After two minutes of equilibration inside the equipment, five measurements, each consisting of at least five runs were recorded.

Surface Area: Using Brunauer Emmet Teller (BET) methodology, the surface area of each CNT/F was measured as described previously [22]. Briefly, samples were degassed in ultrahigh purity (UHP) nitrogen for 30 minutes at $90{ }^{\circ} \mathrm{C}$, and then for 90 minutes at $200{ }^{\circ} \mathrm{C}$. The surface areas were determined by a 5 point BET measurement with UHP nitrogen as the adsorbate and liquid nitrogen as the cryogen.

Zeta Potential: Zeta potentials were measured using a Nano ZS90 instrument (Malvern Instruments, UK). Viscosity of the control medium was previously determined at room temperature using a VS-10 viscometer (Malvern Instruments) and used as the value for calculation of zeta potential. The $\mathrm{pH}$ of all samples was measured using a calibrated electrode. 
Dustiness: Dustiness is a unitless measurement (mass/mass) measured using the Venturi dustiness device as was previously described [20]. This measurement represents a percentage of total ( inhalable) and respirable airborne mass normalized to the quantity of test powder prior to dispersion.

Density: Skeletal density of each CNT/F was determined based on ISO 23145. For tapped density, a 10 $\mathrm{ml}$ graduated cylinder was tared on a calibrated analytical balance and the material was added. To measure tapped density, the container was gently tapped, and the level of the powder was recorded to the nearest $0.1 \mathrm{ml}$. The cylinder with powder was reweighed. Density was calculated as the mass of powder divided by volume. The measurement was replicated three times for each sample and the results are expressed as means \pm standard deviation.

Metal Analysis: ICP-AES was used to measure metal content. Digestion was completed using a microwave digestion system (MARS, CEM). Five mg of each sample and $10 \mathrm{ml}$ of concentrated nitric acid were added to the digestion vessel and were subsequently digested using the following program: maximum power $400,100 \%$ power, ramp $20{ }^{\circ} \mathrm{C} / \mathrm{min}, 600 \mathrm{psi}$, temperature $230{ }^{\circ} \mathrm{C}$, hold time $60 \mathrm{~min}$. Samples were then heated on a hot block to reduce the volume to $1 \mathrm{ml}$. The samples were then brought to a volume of $10 \mathrm{ml}$ using deionized water. Sample digests were analyzed according to NMAM 7300. Metal analysis included aluminum, antimony, arsenic, barium, beryllium, cadmium, calcium, chromium, cobalt, copper, iron, lanthanum, lead, lithium, magnesium, manganese, molybdenum, nickel, phosphorus, potassium, selenium, silver, strontium, tellurium, thallium, tin, titanium, vanadium, yttrium, zinc, and zirconium.

Endotoxin: Endotoxin contamination was measured using the Limulus amebocyte lysate test according to the manufacturer's protocol. The limit of detection was $0.1 \mathrm{EU} / \mathrm{ml}$.

$\underline{P A H}:$ PAH levels were quantified by gas chromatography-mass spectrometry with selected ion monitoring (GC-MS SIM) using method previously described [141]. Briefly, dry samples of CNT/F were extracted in $10 \mathrm{ml}$ methylene chloride with shaking for two minutes. The samples were extracted three 
times and the extracts were combined. The Limit of Quantification (LOQ) and Limit of Detection (LOD) and other details were provided previously [141].

TGA: Thermogravimetric analysis (TGA) was performed to determine the residual ash contents and thermal stability of the materials. Samples were analyzed as previously described using a Q50000IR TGA (TA Instruments Inc., New Castle, DE) [22].

Acellular Oxidative Potential: The acellular oxidative potential of $\mathrm{CNT} / \mathrm{F}$ was determined using ferric reducing ability of serum (FRAS). Serum is a complex mixture consisting of various forms of antioxidants that can quench chemically distinct oxidants. The oxidative potential of the CNT/F was determined by reacting human blood serum (HBS; Sigma-Aldrich, St. Louis, MO; Cat \# P2918) with CNT/F and evaluating the decrease in antioxidants in HBS. The reduction in antioxidant capacity of the serum was quantified by ferric to ferrous ion reduction and formation of a colored ferrous-tripyridyltriazine complex. The decrease in antioxidative capacity in HBS was compared with Trolox, a vitamin E analog. This modified total antioxidant capacity approach has been used to evaluate the oxidative potential of various engineered nanomaterials [157-159].

Human blood serum was rapidly thawed and exposed to $\mathrm{CNT} / \mathrm{F}$ at a concentration of $5 \mathrm{mg} / \mathrm{ml}$ in low protein retention tubes. To properly disperse the nanomaterials, the samples were sonicated for 5 minutes. The dispersed samples were then incubated in the dark at $37{ }^{\circ} \mathrm{C}$ for three hours on an orbital shaker set at $450 \mathrm{RPM}$. The $\mathrm{CNT} / \mathrm{F}$ were removed from serum by centrifuging the mixture at $14,000 \mathrm{~g}$ for three hours. $50 \mu \mathrm{l}$ of the serum supernatant was reacted with $1 \mathrm{ml}$ of the FRAS solution to quantify the level of antioxidant depletion. The FRAS solution is a volume mixture of 10:1:1 consisting of $0.2021 \mathrm{~g}$ of sodium acetic trihydrate and $1.060 \mathrm{ml}$ of glacial acetic acid (Alfa Aesar, Haverhill, MA; Cat \# 36289) in $100 \mathrm{ml}$ of deionized water, $0.0946 \mathrm{~g}$ of TPTZ (2,4,6-tri(2-pyridyl)-s-triazine)(Sigma-Aldrich, Cat \# $\mathrm{T} 1253)$ and $1.2 \mathrm{ml}$ of $1 \mathrm{M} \mathrm{HCl}$ in $30 \mathrm{ml}$ of deionized water and $0.1635 \mathrm{~g}$ of $\mathrm{FeCl}_{3} \cdot 6 \mathrm{H}_{2} \mathrm{O}$ (Sigma-Aldrich, Cat \# 44944) in $30 \mathrm{ml}$ of deionized water respectively. For quantitative comparison of the level of 
antioxidant depletion, Trolox (Sigma-Aldrich, Cat \# 238813) standards were prepared at concentrations of $25-800 \mu \mathrm{M}$ and reacted with the FRAS solution. The change in color was quantified by reading the absorption at $586 \mathrm{~nm}$.

\subsubsection{In vitro Study Design and Methods}

The goal of this study was to investigate the toxic effects of CNT/F on pulmonary epithelial cells. Immortalized human bronchial epithelial cells (BEAS-2B), cells were exposed to several concentrations of each of the nine materials. Changes in cell viability, oxidative stress, and protein production were determined. Additionally, the genotoxicity of these materials was assessed using $\gamma \mathrm{H} 2 \mathrm{AX}$ detection and micronuclei formation. The BEAS-2B cell line was selected as a non-tumorigenic cell line originally derived from human bronchial epithelial cells immortalized by viral transfection[160]. Since their original description, monocultures of these cells have been widely used and accepted by researchers to study genotoxicity and potential lung carcinogenesis of test agents. The BEAS-2B cells have several advantages that have made them suitable cell population for genotoxicity analysis. The cells have a stable karyotype and a low background frequency of micronuclei at early passage[161-163] [45] [19] [46, 47]. These cellular characteristics of the BEAS-2B are in accordance with the OECD guidelines as follows: "Because the background frequency of micronuclei will influence the sensitivity of the assay, it is recommended that cell types with a stable and defined background frequency of micronucleus formation and a stable karyotype be used." Previous investigations have demonstrated that the BEAS-2B cells double every 18 to 20 hours when seeded at $70 \%$ density[46, 47] [45] [162].

Correspondence to Human exposure: The experiments were performed on $\mu \mathrm{g} / \mathrm{ml}$ basis. As the surface area and volume required changes with the cell culture consumable used for the assays, in order to be open and enable future comparative and meta-analysis of the data generated, we have reported the concentrations in $\mu \mathrm{g} / \mathrm{cm}^{2}$ basis alongside the $\mu \mathrm{g} / \mathrm{ml}$. Cellular toxicity and oxidative stress were performed 
at a range of approximately $0-15 \mu \mathrm{g} / \mathrm{cm}^{2}(0-24 \mu \mathrm{g} / \mathrm{ml})$. Micronuclei formation and $\gamma-\mathrm{H} 2 \mathrm{AX}$ were evaluated at 0.009 and $0.9 \mu \mathrm{g} / \mathrm{cm}^{2}$, very much at the lower end of the toxicity range.

Based on Erdely et.al 2013[164], an inhalable elemental carbon mass concentration arithmetic mean of $10.6 \mu \mathrm{g} / \mathrm{m}^{3}$ (geometric mean $4.21 \mu \mathrm{g} / \mathrm{m}^{3}$ ) was found among workers exposed to MWCNT. The concentration equates to a deposited dose of approximately $4.07 \mu \mathrm{g} / \mathrm{d}$ in a human. The in vitro exposure of $0.009 \mu \mathrm{g} / \mathrm{cm}^{2}$, based on an human alveolar surface area of $102 \mathrm{~m}^{2}\left(1.02 \times 10^{6} \mathrm{~cm}^{2}\right)$ [165] corresponds to $9180 \mu \mathrm{g}$ deposited in the human. With estimated $4.07 \mathrm{ug} / \mathrm{d}$ deposited, this would be equivalent to exposure of approximately 2250 days. Assuming 5 days/week of work the 2250 days corresponds to $\sim 9$ years of exposure. The in vitro exposure of $0.9 \mu \mathrm{g} / \mathrm{cm}^{2}$ corresponds to $918,000 \mu \mathrm{g}$ deposited in the human. With estimated $4.07 \mathrm{ug} / \mathrm{d}$ deposited, this would be equivalent to exposure of 225000 days. Assuming 5 days/week of work the 225000 days corresponds to $\sim 900$ years of exposure.

$\underline{C N T / F}$ Dispersion in Cell Culture Media: Aqueous stock suspensions of CNT/F were generated by weighing the dry powder and suspending in well-characterized dispersion medium [DM; $0.6 \mathrm{mg} / \mathrm{ml}$ mouse serum albumin $+0.01 \mathrm{mg} / \mathrm{ml}$ 1,2-dipalmitoyl-sn-glycero-3-phosphotidyl (DPPC) in phosphate-buffered saline (PBS) without calcium and magnesium] [166] at $2 \mathrm{mg} / \mathrm{ml}$ concentration. The stock suspension was sonicated for 5 minutes at $70 \%$ amplitude using a cup horn sonicator (Sonics VibraCell VCX-750 with Cup-type Sonicator; Newton, CT) immersed in continuous flowing cold water. The samples were vortexed intermittently after every minute for 10 seconds. The stock solution at $2 \mathrm{mg} / \mathrm{ml}$ was dispersed in cell culture media by diluting to highest test concentration i.e. $24 \mu \mathrm{g} / \mathrm{ml}$. The CNT/F containing cell culture media was then subjected to probe tip sonication (Branson Sonifer 450, continuous output) for a total of 2 minutes, with 10 second vertexing after every 30 seconds. CNT/F containing cell culture media at 0.024 , 0.24 or $2.4 \mu \mathrm{g} / \mathrm{ml}$ were obtained by serial dilution. 


\subsubsection{Cell culture and cytotoxicity}

Human bronchial epithelial cells (BEAS-2B) were obtained from American type culture collection (ATCC, Manassas, VA) and cultured in Dulbecco's modified Eagle medium (DMEM) supplemented with $10 \%$ heat inactivated fetal bovine serum (R\&D Systems Inc, Minneapolis, MN) and 1\% penicillin Streptomycin (Invitrogen, Carlsbad, CA). Cells were cultured to $70 \%$ confluency in an incubator maintained at $37^{\circ} \mathrm{C}$ and $5 \% \mathrm{CO}_{2}$. Trypsin-EDTA (0.25\%) was used to detach the cells from the culture flasks for sub-culturing. The cells between passage 4-10 were used and these cells had a doubling time of 18-20 hours. For evaluating the cytotoxicity, parallel cultures of cells were seeded at $46,900 \mathrm{cells} / \mathrm{cm}^{2}$ overnight in a 96-well plate and dosed at a concentration of $0.024, .24$ or 2.4 or $24 \mu \mathrm{g} / \mathrm{ml}$ to the CNT/F with MW \#5 (Mitsui-7/MWCNT-7) serving as a positive control. In terms of surface area this corresponds to $0.015,0.15,1.5$ and $15 \mu \mathrm{g} / \mathrm{cm}^{2}$. Parallel cells cultures were exposed to $\mathrm{CNT} / \mathrm{F}$ for $24 \mathrm{~h}$ and challenged with fresh media containing $10 \%$ volume/volume WST-1 cell proliferation reagent (Sigma-Aldrich, Cat \#5015944001). After $2 \mathrm{~h}$ of incubation the WST-1 consumption was recorded by measuring the absorbance at $450 \mathrm{~nm}$ subtracted with absorbance at $660 \mathrm{~nm}$ to account for turbidity/background. Cytotoxicity was evaluated by repeating the experiment on three separate days with each dose tested in quadruplicates each day.

\subsubsection{Oxidative stress}

Intracellular ROS formation after $24 \mathrm{~h}$ post exposure of the CNT/F was assessed using CellROX® Green (Invitrogen). Cells were seeded at 46,900 cells $/ \mathrm{cm}^{2}$ overnight in a 24 -well plate and dosed at a concentration of $0.024,0.24,2.4$, or $24 \mu \mathrm{g} / \mathrm{ml}$ of one of the nine materials tested. In terms of surface area this corresponds to $0.012,0.12,1.2$ and $12 \mu \mathrm{g} / \mathrm{cm}^{2}$. After $24 \mathrm{~h}$ of exposure to CNT/F, cells were detached using Trypsin-EDTA, and washed and incubated with $50 \mu \mathrm{M}$ CellROX for 20 minutes. Cells were washed and fixed by incubating them with $10 \%$ formaldehyde in PBS. The change in CellROX fluorescence was 
captured using a BD LSR II flow cytometer (BD Biosciences, San Diego, CA). The cells were strained through a Flowmi ${ }^{\mathrm{TM}}$ Cell Strainer (Bel-Art Products, Inc. Wayne, NJ) to achieve uniform single cell suspensions and remove any aggregates. The mean fluorescence was determined using FlowJo (FlowJo LLC, Ashland, OR). The experiment was performed on four separate days with each dose tested in triplicates each day. At least 10,000 cells were analyzed per sample in each group

\subsubsection{Protein quantification}

Alteration in the proteins released due to CNT/F exposure was quantified by measuring twentyseven proteins in the supernatants after $24 \mathrm{~h}$ exposure to $0,2.4$ and $24 \mu \mathrm{g} / \mathrm{ml}$ of the CNT/F. In terms of surface area, this corresponds to $0,0.75$ and $7.48 \mu \mathrm{g} / \mathrm{cm}^{2}$. The proteins were measured using a BIO-RAD Bio plex Pro Human Cytokine Grp 1 Panel 27 plex (Bio-Rad Laboratories Inc, CA, Cat \# M500KCAFOY). The 27 proteins measured include cytokine FGF basic, eotaxin, G-CSF, GM-CSF, IFN$\gamma$, IL-1 $\beta$, IL-1ra, IL-2, IL-4, IL-5, IL-6, IL-7, IL-8, IL-9, IL-10, IL-12 (p70), IL-13, IL-15, IL-17A, IP-10, MCP-1 (MCAF), MIP-1 $\alpha$, MIP-1 $\beta$, PDGF-BB, RANTES, TNF- $\alpha$ and VEGF. These proteins are key cytokines, chemokines and growth factors that play an important role in inflammation. The assay sensitivities for these markers ranged from 0.1 to $33.3 \mathrm{pg} / \mathrm{ml}$.

\subsubsection{Double stranded DNA break}

Phosphorylation of $\mathrm{H} 2 \mathrm{~A}$ histone family member $\mathrm{X}(\mathrm{H} 2 \mathrm{AX})$ occurs during repair of DNA breakage and is considered a sensitive marker for double stranded DNA breakage. Flow cytometric evaluation of H2AX phosphorylation was performed as described earlier [167]. Cells were plated on a 12-well plate overnight and challenged with $1.5 \mathrm{ml}$ of CNT/F dispersed in cell culture medium for $24 \mathrm{~h}$. The cells were dosed with $0,0.024$ and $2.4 \mu \mathrm{g} / \mathrm{ml}$ of $\mathrm{CNT} / \mathrm{F}$. In terms of surface area, this corresponds to $0,0.009$ and $0.9 \mu \mathrm{g} / \mathrm{cm}^{2}$. After $24 \mathrm{~h}$ post exposure to $\mathrm{CNT} / \mathrm{F}$, the cells were lifted by trypsinization and fixed using 
$10 \%$ formaldehyde in PBS. Cells were permeabilized with $0.2 \%$ (v/v) Triton X-100 (Sigma-Aldrich) in

PBS for 30 minutes followed by blocking of nonspecific binding by incubating them with $1 \%(\mathrm{w} / \mathrm{v})$ of bovine serum albumin (Sigma-Aldrich) for $1 \mathrm{hr}$. The cells were then incubated overnight with 1:50 dilution of Phospho-Histone H2A.X (Ser139) Rabbit mAb (Alexa fluor 488 conjugated) (Cell Signaling, Beverly, MA). The cells were strained through a Flowmi ${ }^{\mathrm{TM}}$ Cell Strainer (Bel-Art Products, Inc. Wayne, NJ) to achieve uniform single cell suspensions and remove any aggregates. Fluorescence from single cell suspensions was captured using a BD LSR II flow cytometer (BD Biosciences, San Diego, CA). The mean fluorescence was determined using FlowJo (FlowJo LLC, Ashland, OR). The experiment was performed in triplicates and at least 10,000 cells were analyzed per sample in each group.

\subsubsection{Micronucleus assay}

BEAS-2B cells (>97\% viability by trypan blue) were plated at $70 \%$ confluency on a two-well glass chamber slides (Thermo Scientific Nunc Lab-Tek, Waltham, MA; Cat\# 154461) overnight and challenged with $1.5 \mathrm{ml}$ of the $9 \mathrm{CNT} / \mathrm{F}$ dispersed in cell culture medium for $24 \mathrm{~h}$. As outlined in previous investigations, fresh media was added with the test agent to the cultured cells to stimulate cell proliferation. The cells were monitored for cell rounding for $24 \mathrm{~h}$ following the addition of media to assure that mitosis had occurred. Cell rounding is an accepted measure of cell proliferation because most attached cells in culture round up when the cells enter mitosis [168]. The cells were harvested for analysis $24 \mathrm{~h}$ after the addition of fresh media and test agent to avoid growing the cells to confluence. The potency of the test CNT/F was compared to MW \#5 (Mistui-7/MWCNT-7).

Parallel cell cultures were treated with $0,0.024$ and $2.4 \mu \mathrm{g} / \mathrm{ml}$ of the CNT/F, which included MW \#5 (Mistui-7/MWCNT-7) as a reference material known to be genotoxic. In terms of surface area, this corresponds to $0,0.009$ and $0.9 \mu \mathrm{g} / \mathrm{cm}^{2}$. After $24 \mathrm{~h}$ post exposure to $\mathrm{CNT} / \mathrm{F}$, the slides were washed with PBS and fixed with an ice-cold mixture of 3:1 methanol and acetic acid for $30 \mathrm{~min}$ and then stained with 
DAPI (Vector, Burlingame, CA) for nuclear content. The cells were imaged using a laser scanning confocal microscope (LSM 780, CZ Microscopy, Thornwood, NY) using a 60x objective. The complete depth of the cell was captured by taking Z-Sections and the 3D images were converted to $2 \mathrm{D}$ using maximum intensity projection. Photographs of a minimum of 100 cells per slide were taken and the number of micronuclei present was recorded, and the experiment was repeated in triplicate for a minimum of 300 cells per treatment group. Two independent observers that were blinded to the treatment groups recorded the number of micronuclei.

\subsubsection{Statistical analysis}

In vitro assays of cytotoxicity and oxidative stress were analyzed using one-way (particle type) and two-way (particle type by dose) analyses of variance. Post hoc comparisons were evaluated with Fishers LSD test. Some variables were transformed using the natural log prior to analysis to meet the model assumptions of homogeneous variance. Significance was achieved at a $p<0.05$. All analyses were carried out using SAS/STAT version 9.4 for Windows, and JMP statistical software version 12 (SAS, Cary NC).

\subsubsection{Feature selection and principal component analysis}

To permit selection of the minimal number of features among all characterization and L-W-AR properties that could potentially discriminate between each material investigated, feature selection was performed with a random forest-based approach [169] using the "Boruta" algorithm [170] in the R statistical environment [171]. The Boruta algorithm adds randomness to the variables in the dataset by creating shuffled copies of all variables ("shadow features"). "Boruta" iteratively assesses if each variable has a higher Z-score than the maximum Z-score of its shadow features. At each iteration, variables with Zscores lower than shadow features are deemed unimportant and removed subsequently by the algorithm 
to capture all the important, interesting features one might have in the dataset with respect to a dependent variable, in this case each material itself. Then, using traditional, L-W-AR, and combined variables retained after applying the "Boruta" algorithm, principal component analysis (PCA) was performed to identify significant patterns that explained the majority of the variations in the physicochemical properties among the different $\mathrm{CNT} / \mathrm{F}$ materials investigated. PCA was performed using the prcomp command of the R statistical software (R Core Team, 2016) 


\section{CHAPTER 2 TABLES}

Table 2.1. Physical dimensions of CNT/F dispersed in isopropanol.

\begin{tabular}{|c|c|c|c|c|c|c|c|c|c|c|}
\hline & & MW\#1 & MW \#2 & MW \#3 & MW \#4 & MW \#5 & MW \#6 & MW \#7 & CNF \#1 & CNF \#2 \\
\hline \multirow{5}{*}{$\begin{array}{l}\text { Diameter } \\
\text { (nm) }\end{array}$} & $\begin{array}{r}\text { Company Reported } \\
\text { Diameter }(\mathrm{nm})\end{array}$ & $6-9$ & 10 & $10-15$ & $5-30$ & N/A & $70-80$ & 150 & 100 & 150 \\
\hline & $\begin{array}{r}\text { Geometric Mean } \\
(\mathrm{nm} \pm \mathrm{GSD})\end{array}$ & $13 \pm 1$ & $14 \pm 2$ & $20 \pm 2$ & $19 \pm 1$ & $63 \pm 1$ & $28 \pm 2$ & $37 \pm 2$ & $102 \pm 1$ & $103 \pm 1$ \\
\hline & $\begin{array}{r}\text { Arithmetic Mean } \\
(\mathrm{nm} \pm \mathrm{SE})\end{array}$ & $13 \pm 0$ & $16 \pm 1$ & $26 \pm 2$ & $20 \pm 1$ & $67 \pm 2$ & $38 \pm 3$ & $54 \pm 4$ & $110 \pm 3$ & $110 \pm 3$ \\
\hline & Median & 12 & 14 & 19 & 18 & 63 & 25 & 28 & 98 & 100 \\
\hline & $\begin{array}{r}\text { Normal } \\
\text { Distribution }\end{array}$ & Lognormal & & & & & & & & Lognormal \\
\hline \multirow{6}{*}{ Length $(\mu \mathrm{m})$} & $\begin{array}{r}\text { Company Reported } \\
\text { Length }(\mu \mathrm{m})\end{array}$ & 5 & N/A & $0.1-10$ & 100 & N/A & N/A & N/A & $50-200$ & $50-200$ \\
\hline & $\begin{array}{r}\text { Geometric Mean } \\
(\mu \mathrm{m} \pm \mathrm{GSD}) \\
\end{array}$ & $0.67 \pm 1.81$ & $1.34 \pm 2.21$ & $1.10 \pm 2.00$ & $1.41 \pm 1.97$ & $4.39 \pm 2.07$ & $2.05 \pm 2.53$ & $2.88 \pm 4.26$ & $3.64 \pm 2.36$ & $2.16 \pm 2.31$ \\
\hline & $\begin{array}{r}\text { Arithmetic Mean } \\
(\mu \mathrm{m} \pm \mathrm{SE})\end{array}$ & $0.80 \pm 0.03$ & $1.79 \pm 0.10$ & $1.28 \pm 0.07$ & $1.84 \pm 0.13$ & $5.62 \pm 0.29$ & $3.42 \pm 0.37$ & $7.64 \pm 0.78$ & $5.23 \pm 0.36$ & $3.20 \pm 0.28$ \\
\hline & Median & 0.6607 & 1.5437 & 1.0148 & 1.2896 & 4.547 & 2.1503 & 2.3781 & 3.7273 & 2.0003 \\
\hline & Length Range & $0.1-3.6$ & $0.2-50.9$ & $0.1-8.5$ & $0.3-20.6$ & $1.2-25.8$ & $0.3-37.3$ & $0.1-49.1$ & $0.3-37.6$ & $0.4-42.7$ \\
\hline & $\begin{array}{r}\text { Normal } \\
\text { Distribution } \\
\end{array}$ & Lognormal & Lognormal & Lognormal & & & Lognormal & & Lognormal & Lognormal \\
\hline Aspect Ratio & $\begin{array}{r}\text { Aspect Ratio } \\
(\text { GeoMean } \pm \text { GSD })\end{array}$ & $53 \pm 2$ & $96 \pm 2$ & $50 \pm 2$ & $76 \pm 2$ & $69 \pm 2$ & $73 \pm 2$ & $78 \pm 3$ & $36 \pm 2$ & $21 \pm 2$ \\
\hline
\end{tabular}


Table 2.2. Hydrodynamic diameter, zeta potential, and two-dimensional sizing of CNT/F agglomerates dispersed in physiologic dosing media.

\begin{tabular}{|c|c|c|c|c|c|c|c|c|c|c|c|}
\hline & \\
\hline & & MW \#1 & $\begin{array}{c}\text { MW \#2 } \\
\text { Small } \\
\text { Agglomerates }\end{array}$ & $\begin{array}{c}\text { MW \#2 } \\
\text { Large } \\
\text { Agglomerates }\end{array}$ & MW \#3 & MW \#4 & MW \#5 & MW \#6 & MW \#7 & CNF \#1 & CNF \#2 \\
\hline \multirow{3}{*}{$\begin{array}{c}\text { Spherical } \\
\text { Agglomerates }\end{array}$} & $\%$ Spherical Agglomerated & 87 & 0 & 0 & 83 & 1 & 4 & 0 & 0 & 0 & 0 \\
\hline & $\begin{array}{r}\text { Spherical Agglomerate Mean } \\
\text { Diameter } \\
\mu \mathrm{m} \pm \mathrm{SE}\end{array}$ & $1.28 \pm 0.16$ & $\mathrm{~N} / \mathrm{A}$ & $\mathrm{N} / \mathrm{A}$ & $0.81 \pm 0.10$ & $\mathrm{~N} / \mathrm{A}$ & $\mathrm{N} / \mathrm{A}$ & $\mathrm{N} / \mathrm{A}$ & $\mathrm{N} / \mathrm{A}$ & $\mathrm{N} / \mathrm{A}$ & $\mathrm{N} / \mathrm{A}$ \\
\hline & $\begin{array}{r}\text { Spherical Agglomerate Diameter } \\
\text { Geometric Mean } \\
\mu \mathrm{m}(\mathrm{GSD})\end{array}$ & $0.96(2.01)$ & $\mathrm{N} / \mathrm{A}$ & $\mathrm{N} / \mathrm{A}$ & $0.66(1.84)$ & $\mathrm{N} / \mathrm{A}$ & $\mathrm{N} / \mathrm{A}$ & $\mathrm{N} / \mathrm{A}$ & $\mathrm{N} / \mathrm{A}$ & $\mathrm{N} / \mathrm{A}$ & $\mathrm{N} / \mathrm{A}$ \\
\hline \multirow{5}{*}{$\begin{array}{c}\text { Bundled } \\
\text { Agglomerates }\end{array}$} & $\%$ Bundle Agglomerates/Singlets & 13 & $\mathrm{~N} / \mathrm{A}$ & $\mathrm{N} / \mathrm{A}$ & 17 & 99 & 96 & 100 & 100 & 100 & 100 \\
\hline & $\begin{array}{r}\text { Bundle Agglomerate Mean Length } \\
\mu \mathrm{m} \pm \mathrm{SE}\end{array}$ & $1.90 \pm 0.37$ & $3.80 \pm 0.38$ & $49.55 \pm 3.58$ & $1.11 \pm 0.25$ & $3.77 \pm 0.35$ & $6.27 \pm 0.44$ & $9.47 \pm 1.26$ & $11.32 \pm 1.08$ & $9.30 \pm 1.07$ & $2.96 \pm 0.36$ \\
\hline & $\begin{array}{r}\text { Bundle Agglomerate Geometric } \\
\text { Mean Length } \\
\mu \mathrm{m}(\mathrm{GSD})\end{array}$ & $1.66(1.71)$ & $3.09(1.82)$ & $47.94(1.31)$ & $0.72(2.98)$ & $2.92(2.05)$ & $5.17(1.94)$ & $5.90(2.57)$ & $7.91(2.49)$ & $6.18(2.49)$ & $2.11(2.25)$ \\
\hline & $\begin{array}{r}\text { Bundle Agglomerate Mean } \\
\text { Diameter } \\
\mu \mathrm{m} \pm \mathrm{SE}\end{array}$ & $0.38 \pm 0.12$ & $0.03 \pm 0.00$ & $9.50 \pm 2.24$ & $0.03 \pm 0.00$ & $0.10 \pm 0.01$ & $0.13 \pm 0.01$ & $0.08 \pm 0.01$ & $0.10 \pm 0.01$ & $0.21 \pm 0.01$ & $0.12 \pm 0.01$ \\
\hline & $\begin{array}{r}\text { Bundle Agglomerate Diameter } \\
\text { Geometric Mean } \\
\mu \mathrm{m}(\mathrm{GSD}) \\
\end{array}$ & $0.18(4.20)$ & $0.03(1.60)$ & $6.99(2.24)$ & $0.03(1.56)$ & $0.08(1.89)$ & $0.11(1.07)$ & $0.07(2.09)$ & $0.09(1.64)$ & $0.19(1.42)$ & $0.11(1.63)$ \\
\hline \multicolumn{2}{|l|}{\begin{tabular}{|l|} 
Hydrodynamic \\
Diameter (nm) \\
\end{tabular}} & $660 \pm 19$ & \multicolumn{2}{|c|}{$771 \pm 33$} & $608 \pm 30$ & $478 \pm 24$ & $504 \pm 15$ & $714 \pm 24$ & $652 \pm 26$ & $615 \pm 19$ & $664 \pm 18$ \\
\hline \multicolumn{2}{|c|}{ Zeta Potential (pH 7.3) } & $-10.4 \pm 0.4$ & \multicolumn{2}{|c|}{$-12.1 \pm 0.6$} & $-11.1 \pm 0.06$ & $-12.0 \pm 0.4$ & $-13.5 \pm 0.8$ & $-11.8 \pm 0.6$ & $-13.2 \pm 0.5$ & $-11.1 \pm 0.8$ & $-11.3 \pm 0.5$ \\
\hline
\end{tabular}


Table 2.3. Results of additional particle characterization of CNT/F.

\begin{tabular}{|c|c|c|c|c|c|c|c|c|c|c|}
\hline & & MW \#1 & MW \#2 & MW \#3 & MW \#4 & MW \#5 & MW \#6 & MW \#7 & CNF \#1 & CNF \#2 \\
\hline $\begin{array}{l}\text { Surface Area } \\
\left(\mathrm{m}^{2} / \mathrm{g} \pm \mathrm{SD}\right)\end{array}$ & & $237.7 \pm 1.0$ & $211.9 \pm 1.8$ & $218.6 \pm 1.2$ & $99.4 \pm 1.1$ & $25.2 \pm 0.4$ & $25.4 \pm 0.4$ & $24.7 \pm 0.4$ & $29.4 \pm 0.2$ & $18.0 \pm 0.2$ \\
\hline \multirow{2}{*}{ Dustiness } & $\begin{array}{r}\text { Dustiness Total } \\
(\%)\end{array}$ & 3.8 & 2.9 & 0.3 & 0.5 & 14.0 & 0.2 & 0.2 & 4.9 & ND \\
\hline & $\begin{array}{r}\text { Dustiness } \\
\text { Respirable (\%) }\end{array}$ & 0.84 & 1.10 & 0.20 & 0.20 & 2.40 & 0.08 & 0.09 & 1.40 & ND \\
\hline \multirow{2}{*}{ Density } & $\begin{array}{r}\text { Bulk Density } \\
(\mathrm{g} / \mathrm{cm} 3) \\
\end{array}$ & 0.087 & 0.007 & 0.082 & 0.169 & 0.007 & 0.075 & 0.061 & 0.020 & 0.032 \\
\hline & $\begin{array}{r}\text { Tapped Density } \\
(\mathrm{g} / \mathrm{cm} 3)\end{array}$ & 0.119 & 0.008 & 0.095 & 0.222 & 0.010 & 0.095 & 0.073 & 0.028 & 0.045 \\
\hline Endotoxin & & BLD & BLD & BLD & BLD & BLD & BLD & BLD & BLD & BLD \\
\hline PAH & & BLD & BLD & BLD & BLD & BLD & BLD & BLD & BLD & BLD \\
\hline \multirow{2}{*}{ Metal Catalyst } & $\% \mathrm{Fe}$ & 0.317 & 1.725 & 1.603 & 3.423 & 0.270 & 5.006 & 6.169 & 1.168 & 1.142 \\
\hline & $\% \mathrm{Al}$ & 0.310 & 0.028 & 2.116 & 0.019 & N/A & 0.035 & N/A & 0.006 & 0.013 \\
\hline \multirow{2}{*}{ TGA } & $\begin{array}{r}\text { TGA - Avg onset } \\
\text { oxidation, }{ }^{\circ} \mathrm{C}\end{array}$ & $550 \pm 2$ & $603 \pm 2$ & $575 \pm 0$ & $560 \pm 2$ & $735 \pm 2$ & $581 \pm 0$ & $592 \pm 0$ & $593 \pm 0$ & $694 \pm 0$ \\
\hline & $\begin{array}{r}\text { TGA - Mean } \\
\text { Residual Ash, \% }\end{array}$ & $1.74 \pm 0.01$ & $3.98 \pm 0.26$ & $8.21 \pm 0.26$ & $4.75 \pm 0.07$ & $1.11 \pm 0.28$ & $7.88 \pm 0.15$ & $8.95 \pm 0.29$ & $1.79 \pm 0.12$ & $2.21 \pm 0.16$ \\
\hline $\begin{array}{l}\text { Anti-oxidative } \\
\text { Capacity }\end{array}$ & $\%$ & $64.53 \pm 23.91$ & $75.41 \pm 25.66$ & $76.34 \pm 27.37$ & $88.17 \pm 28.40$ & $91.49 \pm 21.37$ & $84.78 \pm 25.74$ & $77.27 \pm 19.96$ & $100.12 \pm 22.69$ & $99.80 \pm 24.19$ \\
\hline
\end{tabular}




\section{CHAPTER 2 FIGURES}

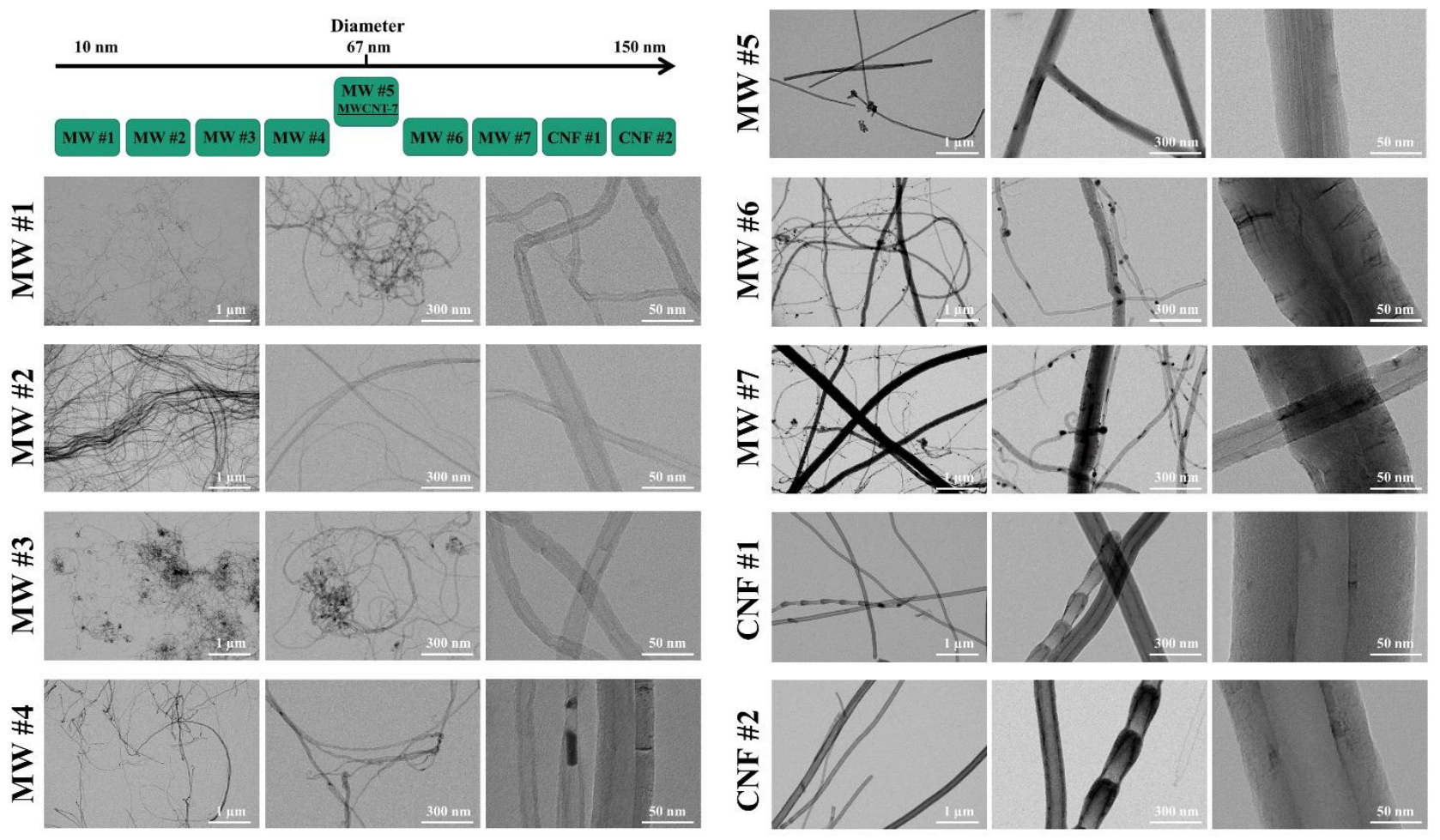

Figure 2.1: Schematic of material diameter and TEM images of CNT/F. Materials selection was based upon company reported diameter ranging from $6-150 \mathrm{~nm}$ in diameter to ensure a full range of materials were included in this study and the material arrangement is depicted in the upper left corner. These materials were identified as MW \#1-7 and CNF \#1-2. A well-studied benchmark material, MWCNT7/Mitsui-7, was included in this study as MW \#5. Materials were dispersed in isopropanol and placed onto a TEM grid to measure physical dimensions. Representative images of each material were selected with scale bars representing $1 \mu \mathrm{m}, 300 \mathrm{~nm}$, and $50 \mathrm{~nm}$ from left to right. 

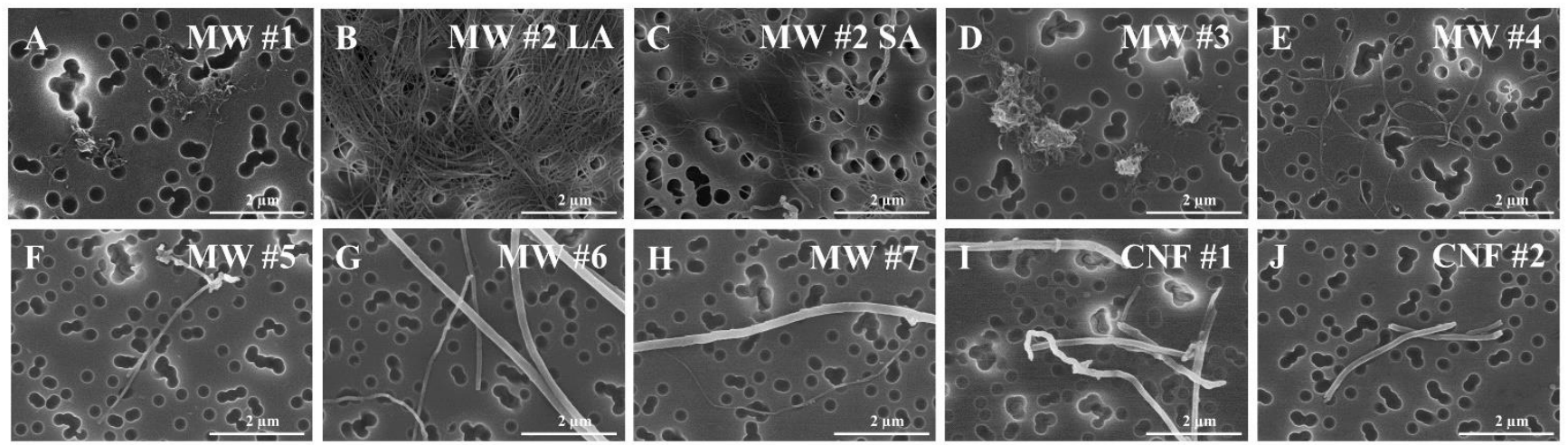

Figure 2.2: Representative scanning electron microscopy images of CNT/F in DM to measure twodimensional agglomerate sizes. $\mathrm{LA}=$ large agglomerates and $\mathrm{SA}=$ small agglomerates. 


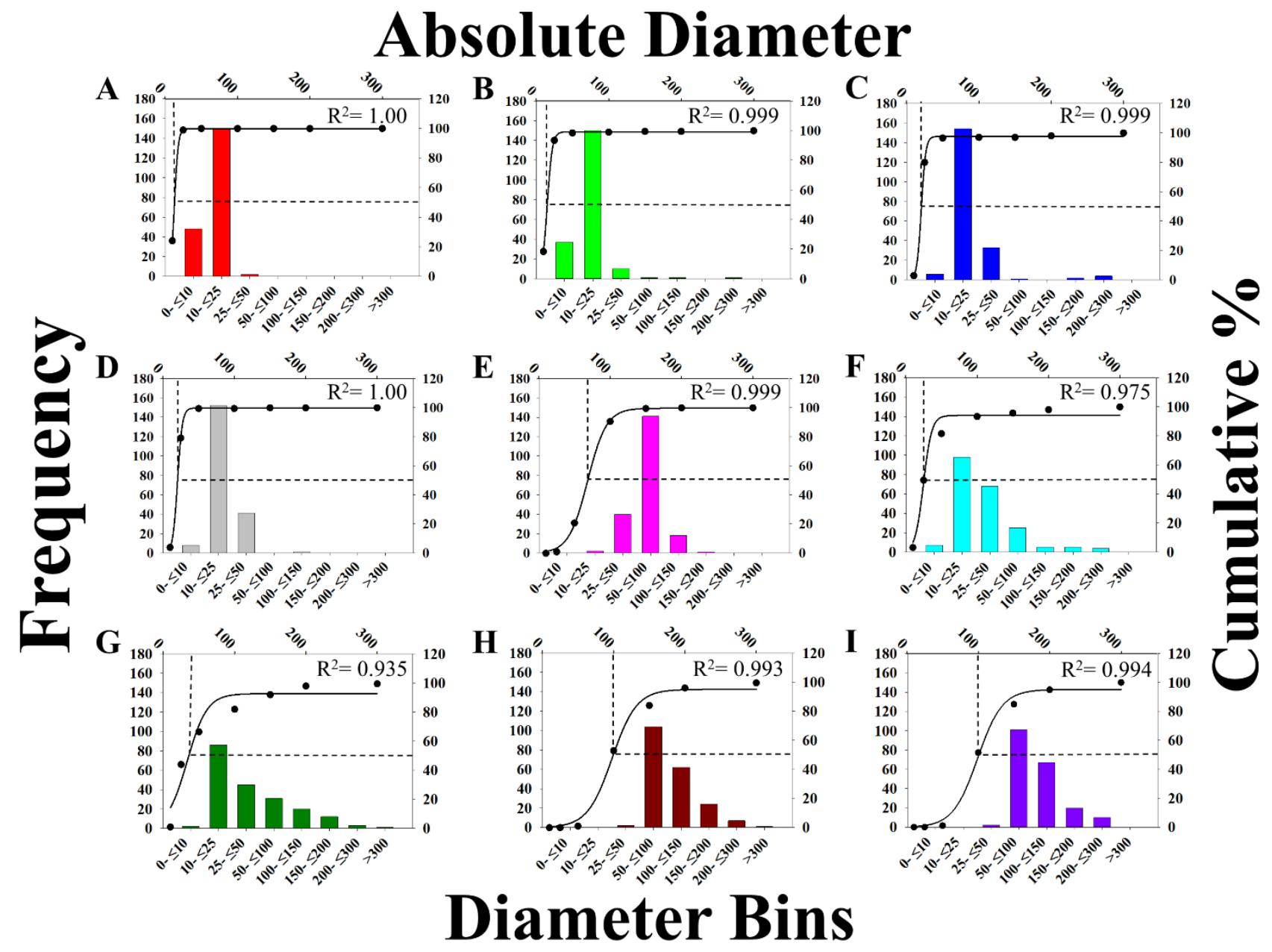

MW \#1 $\rightleftarrows$ MW \#2 MW \#3

MW \#4 $2 \mathrm{MW} \# 5 \square \mathrm{MW}$ \#6

MW \#7

CNF \#1

$\mathrm{CNF} \# 2$

Figure 2.3. Distributions of CNT/F diameter. Particles were binned according to size along the lower $x-$ axis with frequency on the left y-axis. Additionally, percentage of accumulation is graphed on the right yaxis with the absolute diameter along the upper $\mathrm{x}$-axis. The overlay line was 3 parameter sigmoidal curve of best fit with the point of $50 \%$ accumulation indicated with dotted lines. Sizes are for particles in isopropanol suspension. 


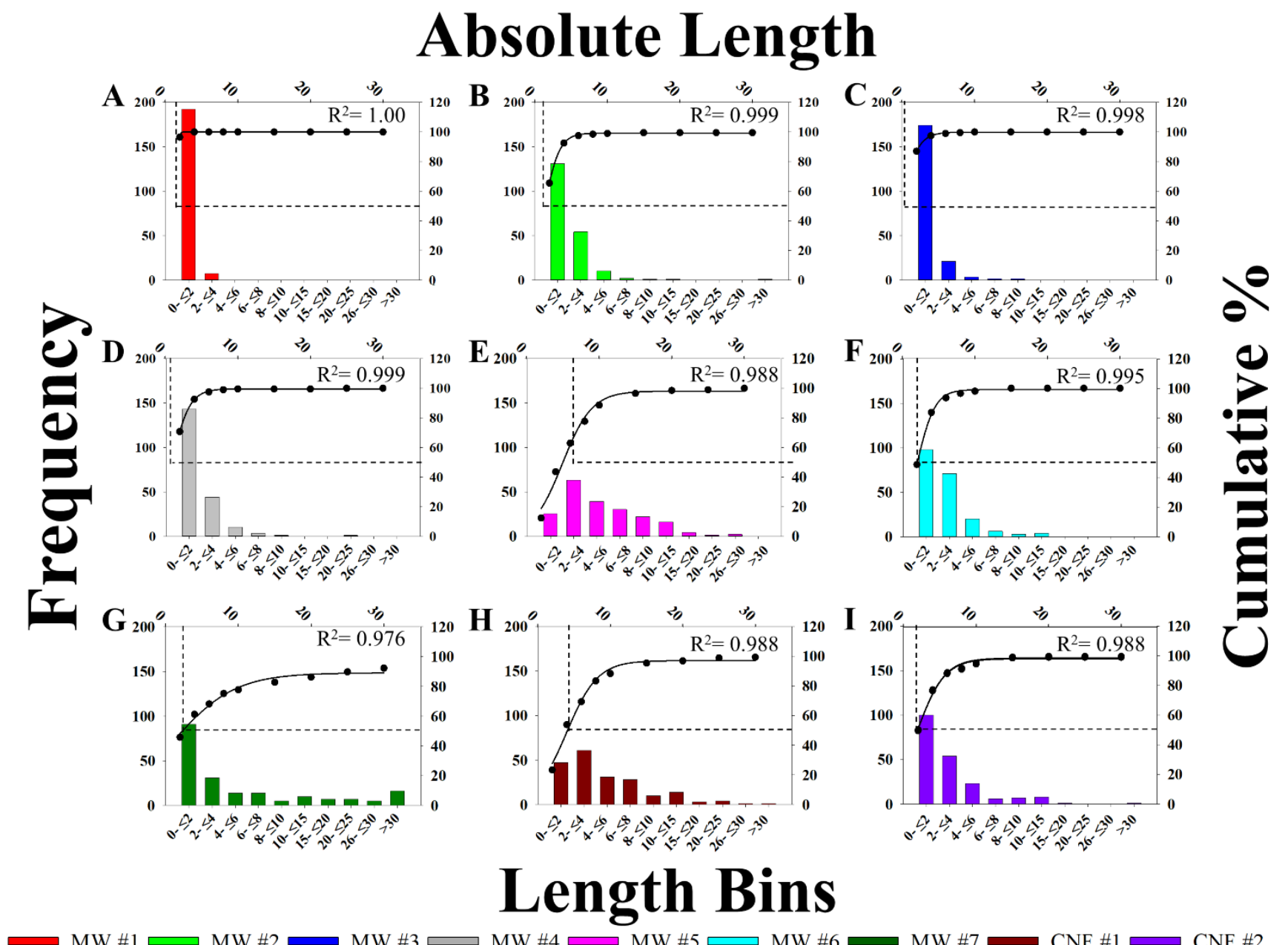

Figure 2.4. Distributions of CNT/F length. Particles were binned according to size along the lower $\mathrm{x}$-axis with frequency on the left y-axis. Additionally, percentage of accumulation is graphed on the right y-axis with absolute length along the upper x-axis. The overlay line is 3 parameter sigmoidal curve of best fit with the point of $50 \%$ accumulation indicated with dotted lines. Sizing was for particles in isopropanol suspension. 


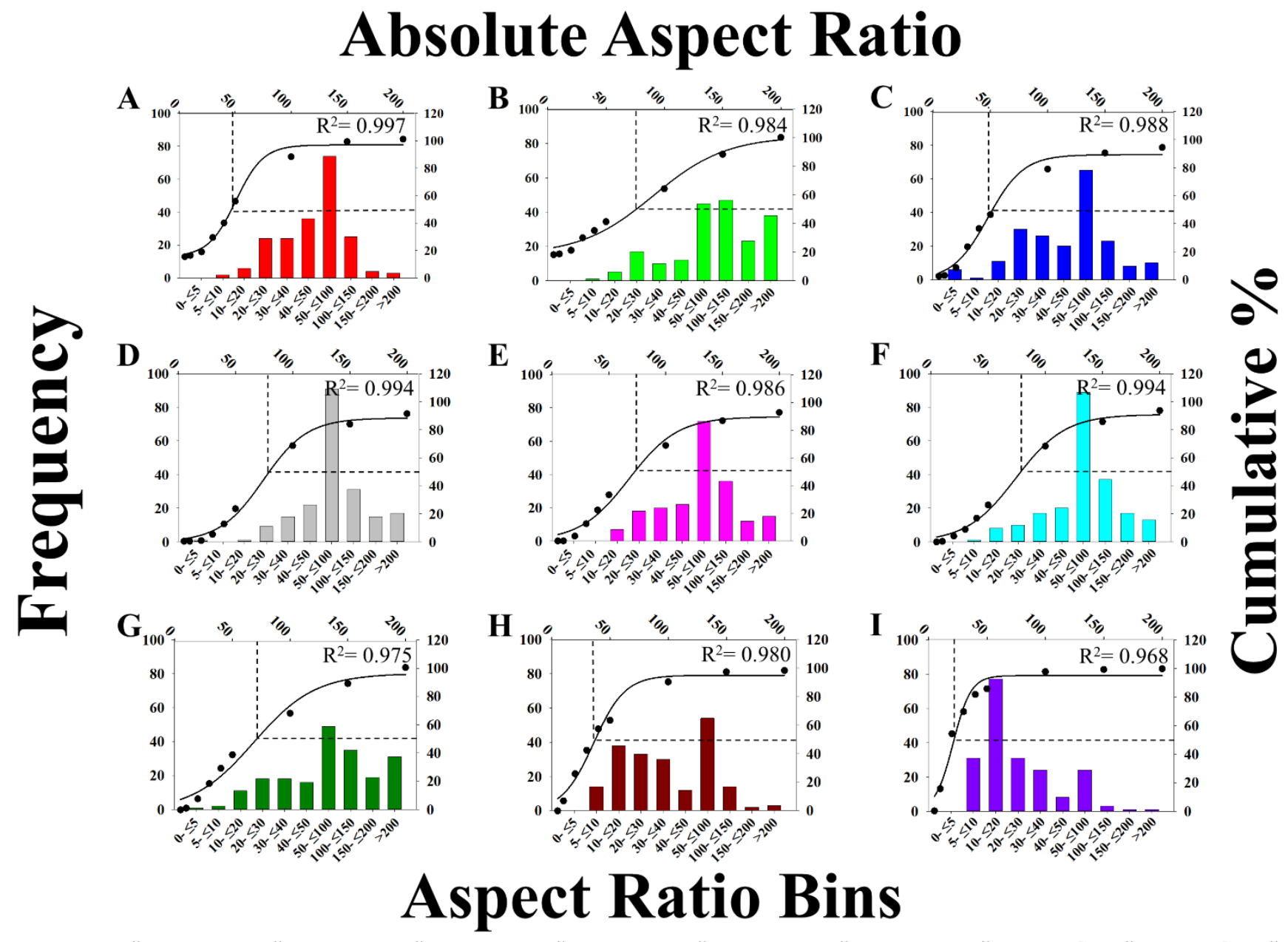

Figure 2.5. Distributions of $\mathrm{CNT} / \mathrm{F}$ aspect ratio. Particles were binned according to size along the lower $\mathrm{x}$-axis with frequency on the left $\mathrm{y}$-axis. Additionally, percentage of accumulation is graphed on the right $\mathrm{y}$-axis with absolute aspect ratio along the upper $\mathrm{x}$-axis. The overlay line is 3 parameter sigmoidal curve of best fit with the point of $50 \%$ accumulation indicated with dotted lines. Sizing was for particles in isopropanol suspension. 

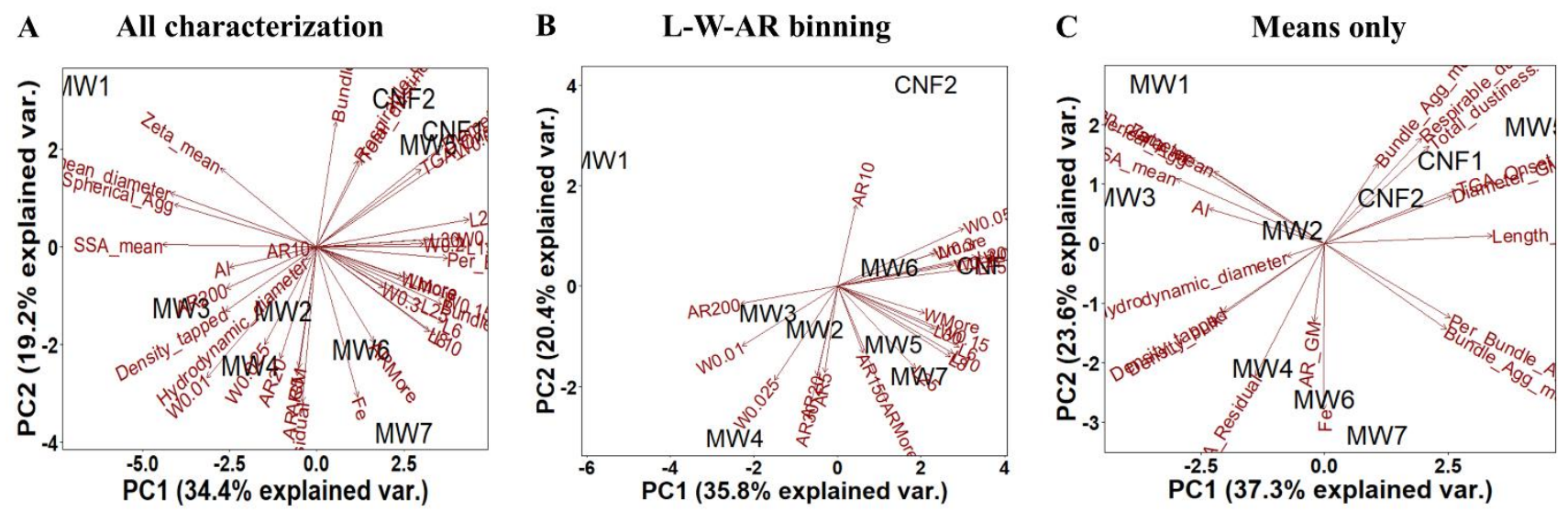

Figure 2.6. Principal component analysis (PCA) of different $\mathrm{CNT} / \mathrm{F}$ materials comparing 'all characterization' parameters (A), length - diameter - aspect ratio physical dimension (B; L-W-AR binning) and means only (C) physicochemical characteristics. The first two principal components (PC), PC1 and PC2, define the $\mathrm{x}$ - and y-axes of the scatter plots, respectively. The distance between two materials reflects the proximity in physicochemical properties between them. PC1, PC2 and PC3 together accounted for $\sim 71 \%, 68 \%$ and $82 \%$ of the contribution to the variance in the case of all characterization, L-W-AR binning and means only, respectively. The scatter plot of the PCA along with vectors depicting the loadings of variables is shown. 
A

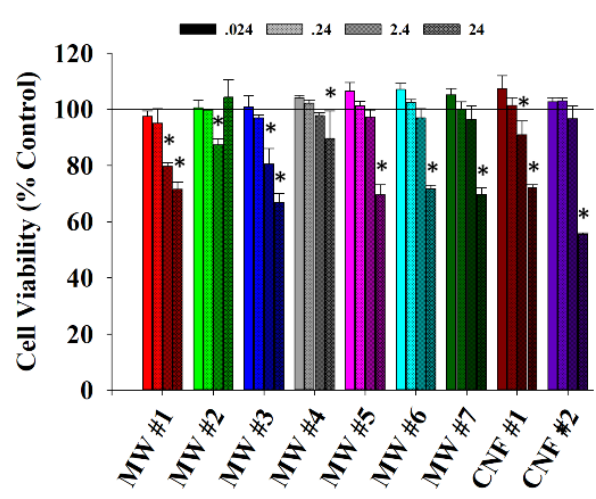

B

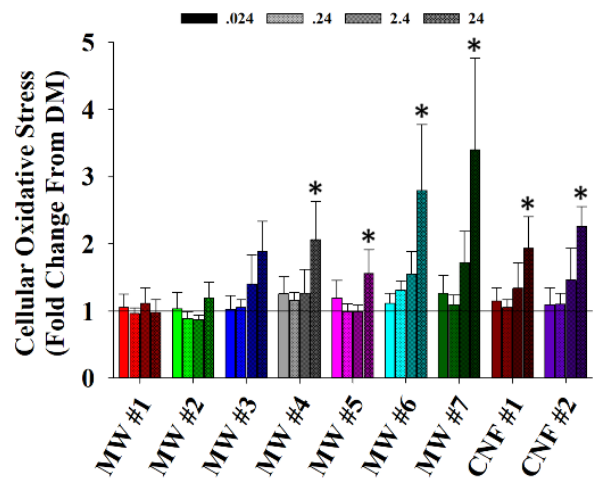

C

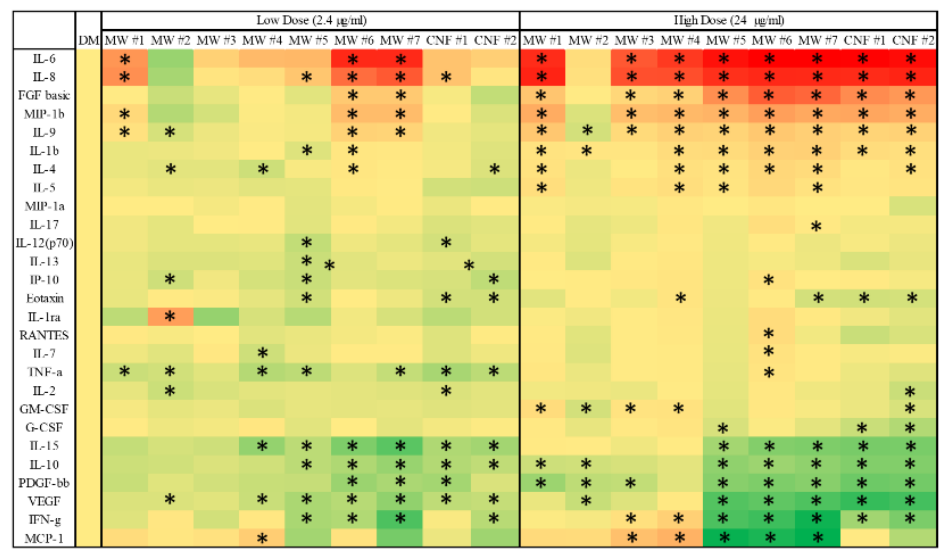

$-0.2$

Figure 2.7. Toxicity assessment of BEAS-2B cells exposed to CNT/F. (A) WST-1 cell proliferation assay was used to assess the viability of BEAS-2B cells following exposure to increasing concentrations (0.024$24 \mu \mathrm{g} / \mathrm{ml}$ ) of CNT/F. The dose at which the particle significantly reduced cell viability is indicated with an asterisk $(\mathrm{p}<0.05)$. (B) Oxidative stress was measured using the CellROX assay. $* \mathrm{p}<0.05$ fold change vs. control cells represented as a reference line. (C) Protein secretions from cells exposed to 2.4 or 24 $\mu \mathrm{g} / \mathrm{ml}$ of various CNT/F for $24 \mathrm{~h}$ represented as heat maps of fold change from controls with no exposure. Significant changes from control cells were indicated with an asterisk $(* \mathrm{p}<0.05)$. Log fold change was represented by color with green indicating a decrease in protein concentration and red indicating an increase on a scale of -0.2 to $1 .\left({ }^{*} \mathrm{p}<0.05\right)$. 

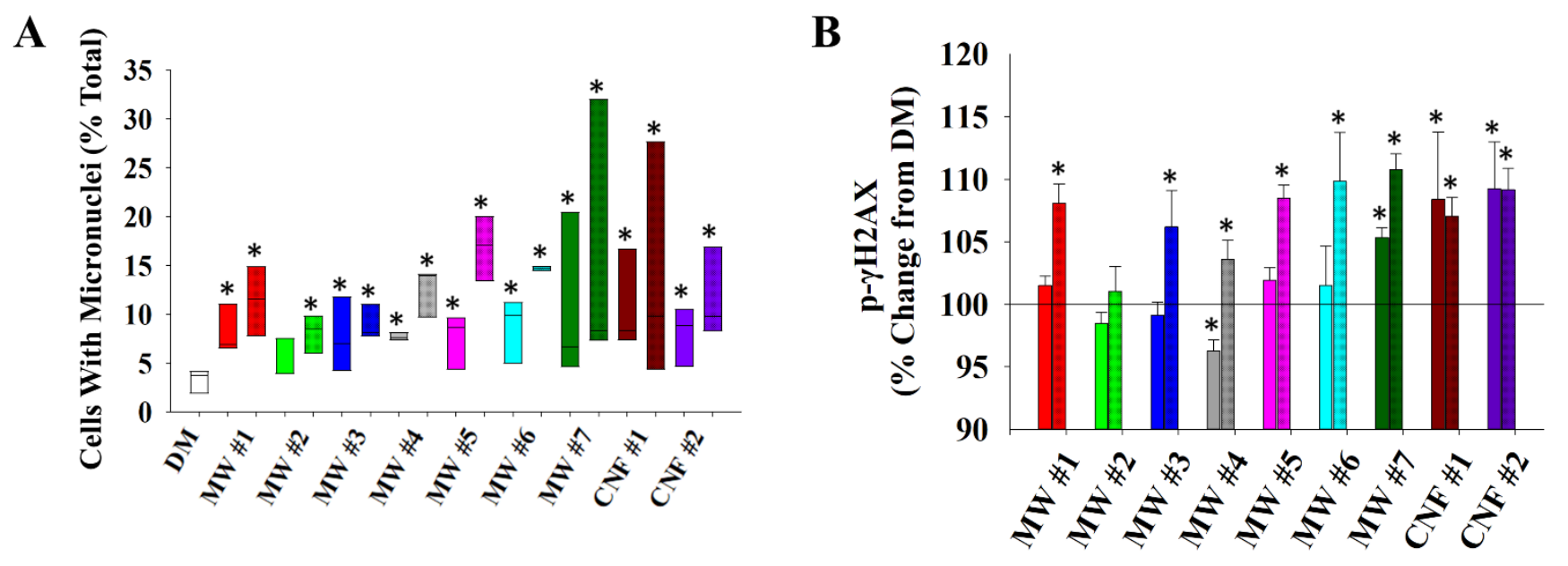

Figure 2.8. Genotoxicity assessments. (A) Cells with micronuclei were quantified and presented as percentage of total cells at treatments of 0.024 and $2.4 \mu \mathrm{g} / \mathrm{ml}$. (B) Inference into double stranded DNA breaks were quantified via detection of $\gamma \mathrm{H} 2 \mathrm{AX}$. Percentage change from DM is presented on the $\mathrm{y}$-axis. ${ }^{*} \mathrm{p}<0.05$ represents significant change from control. 


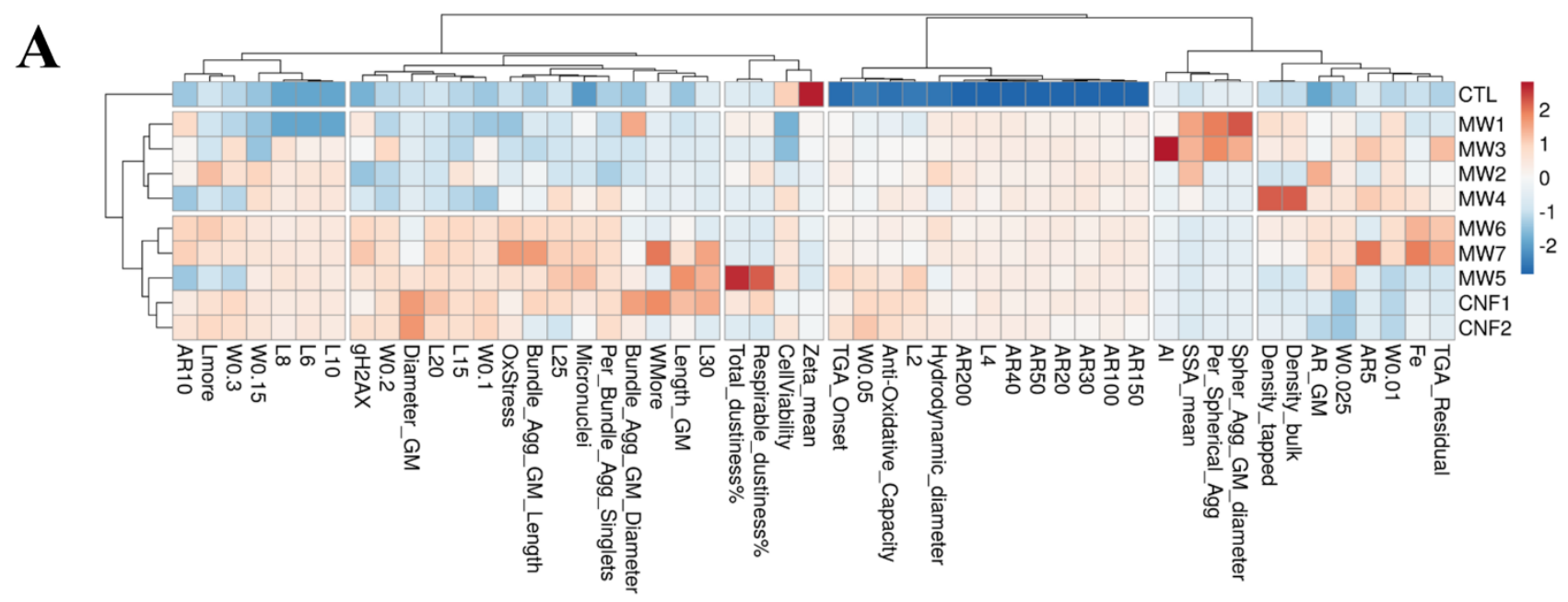

B

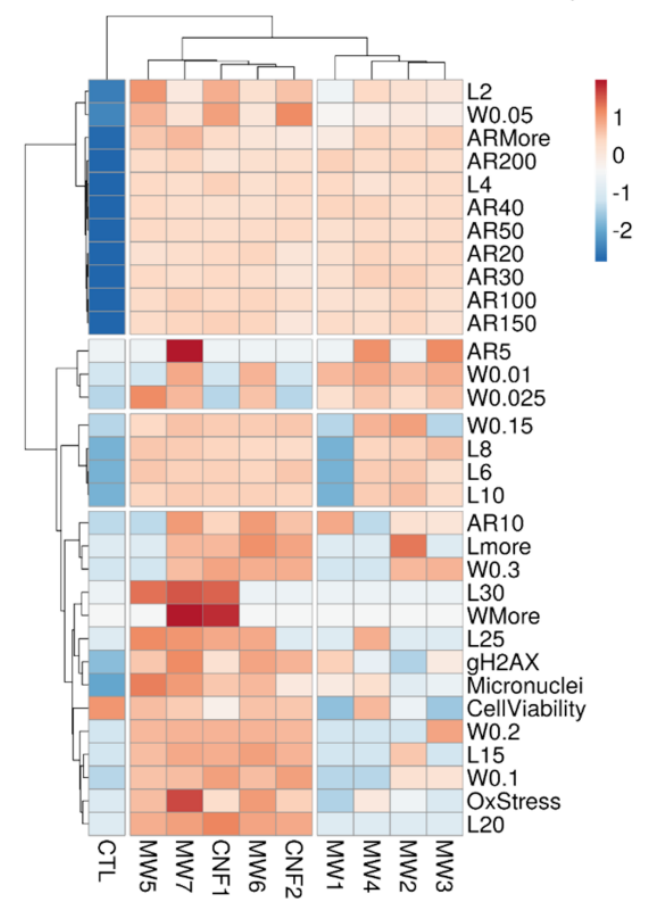

C

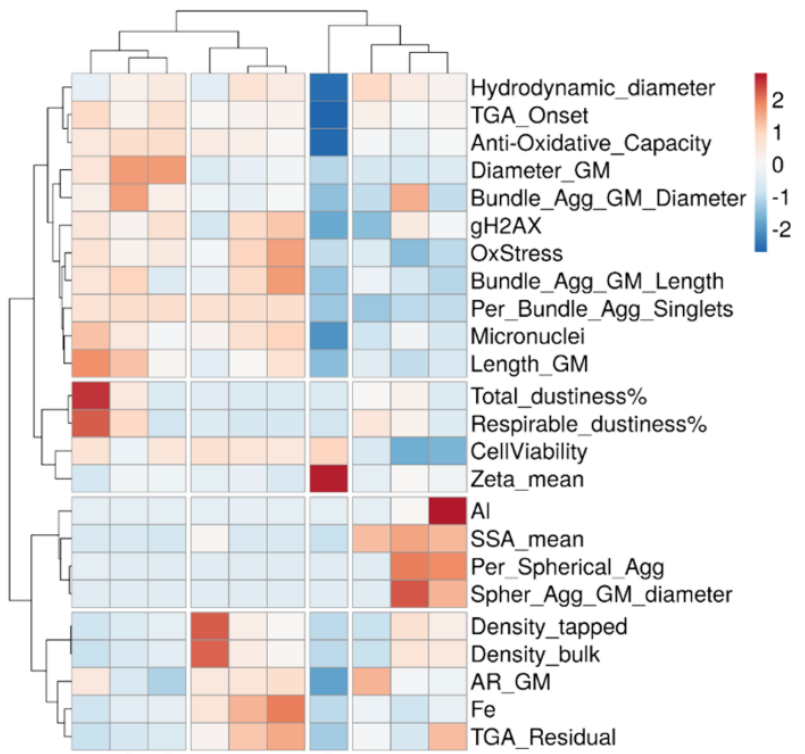

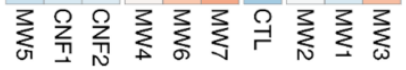

Figure 2.9. Clustering of physicochemical characteristics with the epithelial cell toxicity outcomes of cell viability, cellular oxidative stress, $\gamma \mathrm{H} 2 \mathrm{AX}$ and micronuclei formation. Toxicity outcomes compared to (A) all characteristics, (B) L-W-AR binning, and (C) means only were presented. 


\section{CHAPTER 2 SUPPLEMENTARY TABLES AND FIGURES}

Supplementary Table 2.1 Supernatant protein concentrations following CNT/F exposure in BEAS-2B cells

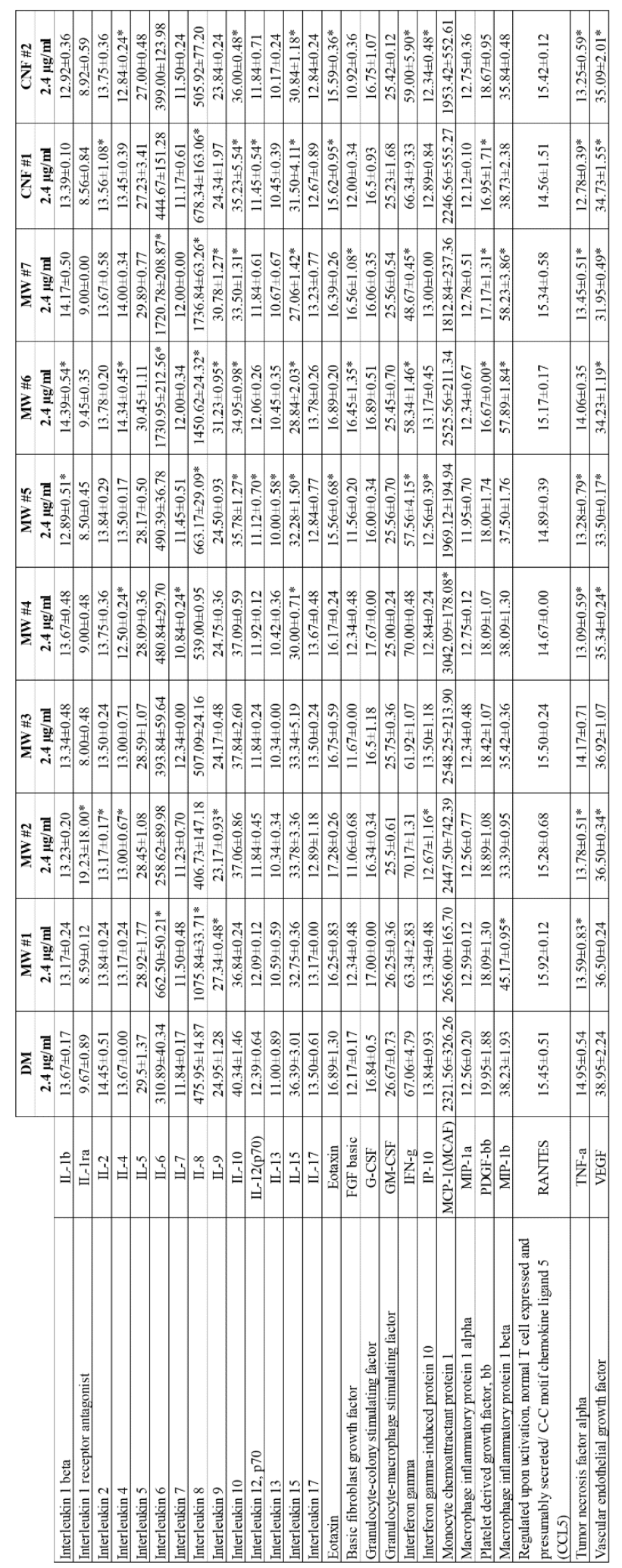




\section{Supplementary Table 2.1 Continued}

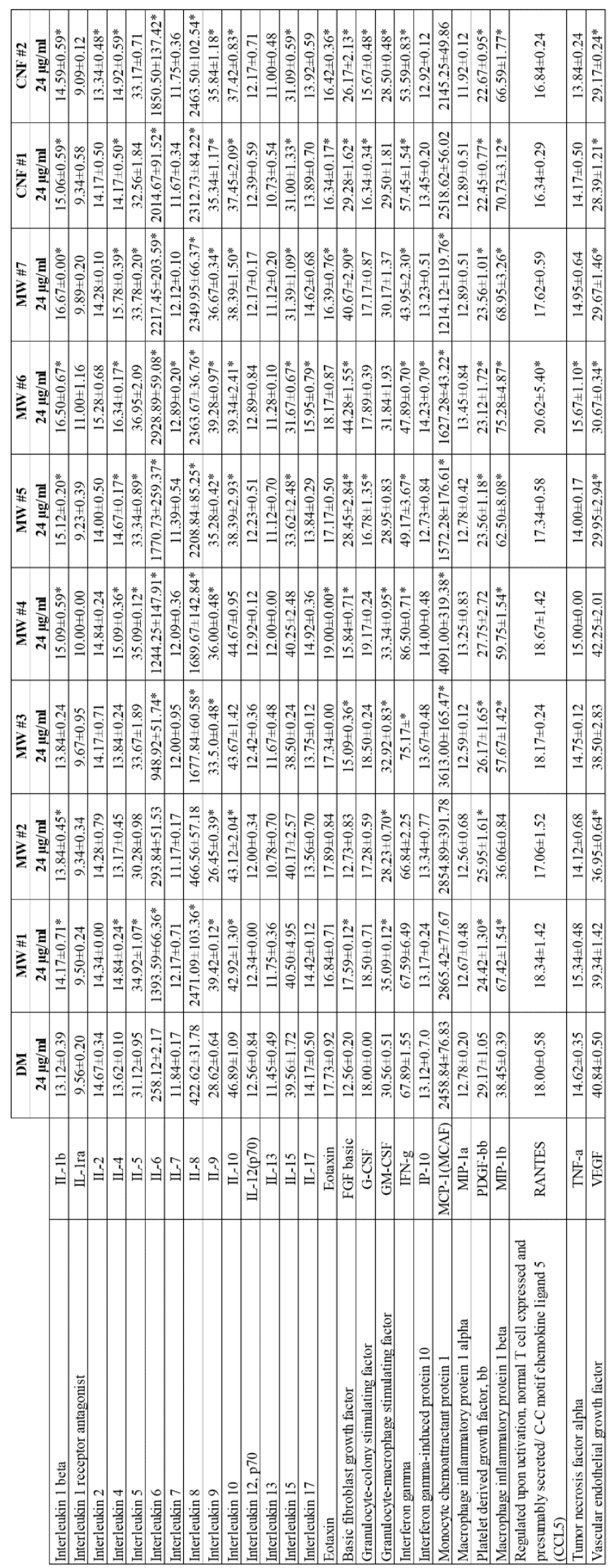



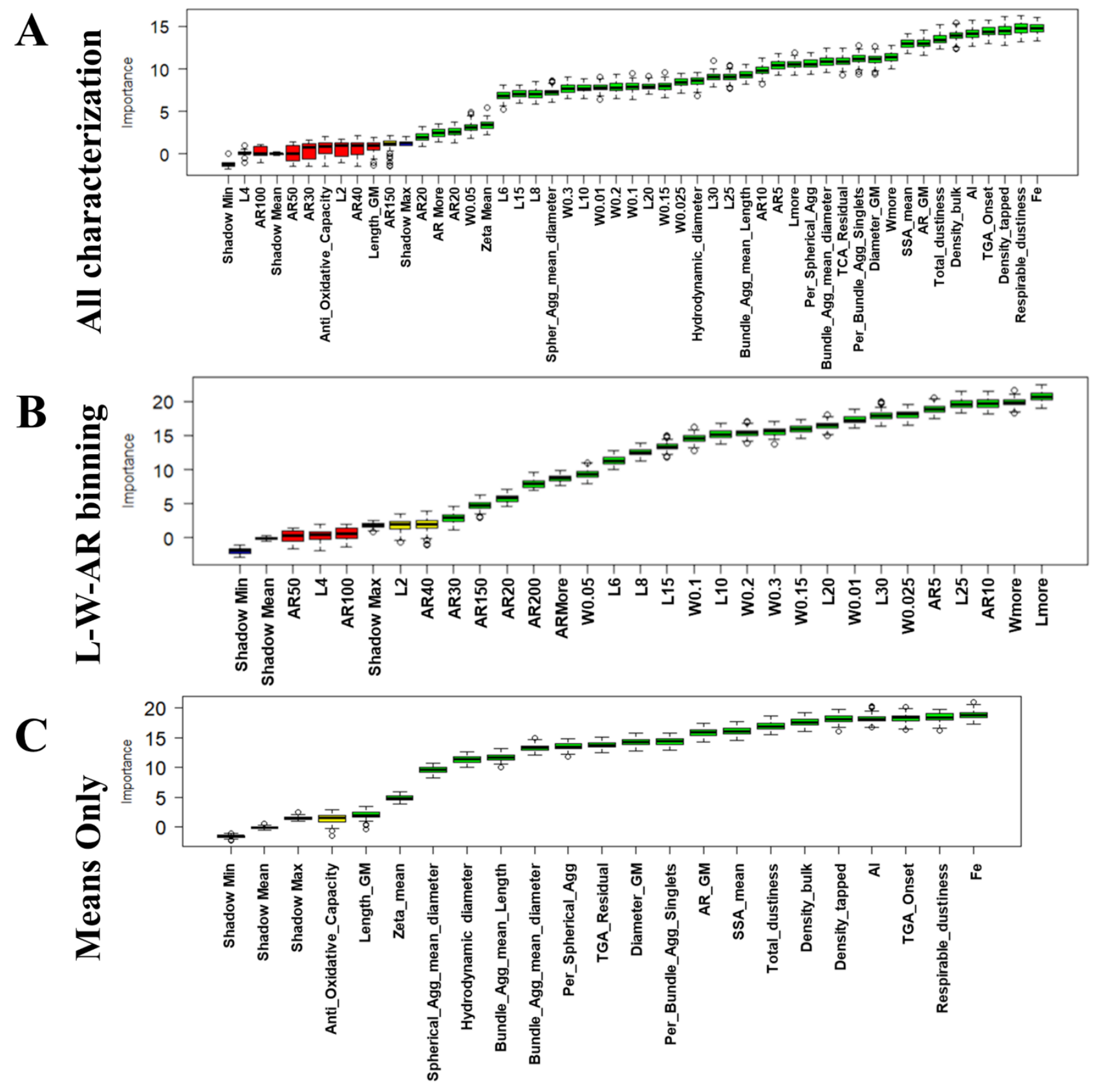

Supplementary Figure 2.1 Variable importance plots for all characterization (A), L-W-AR binning (B), and means only (C) 

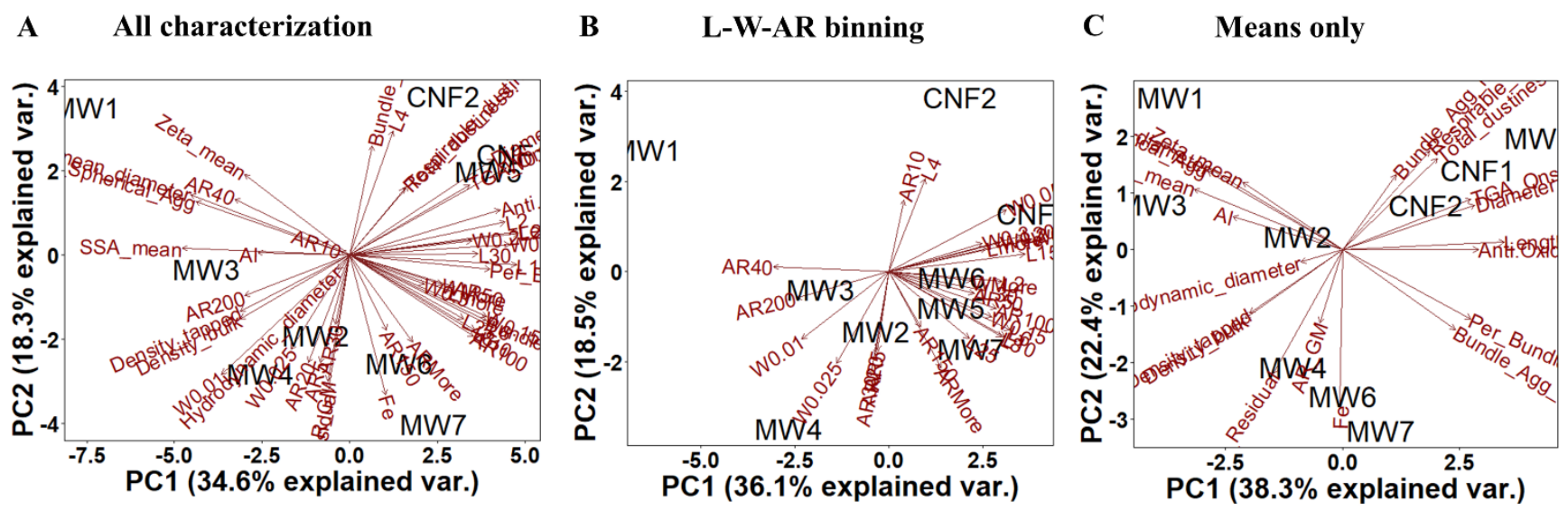

Supplementary Figure 2.2 Principle component analysis plots without feature selection plots for all characterization (A), L-W-AR binning (B), and means only (C) 
A

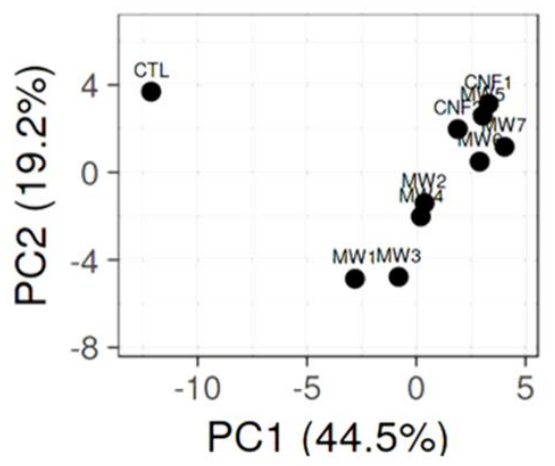

B L-W-AR binning

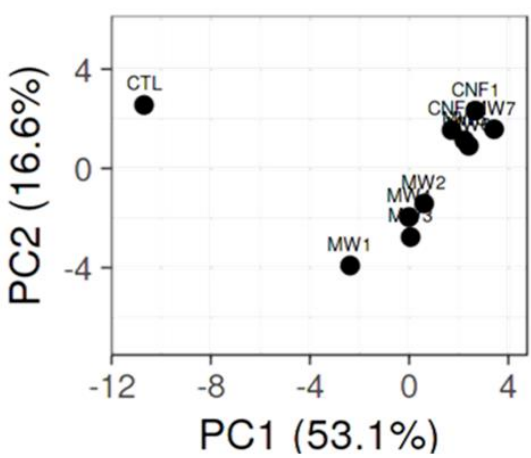

C

Means only

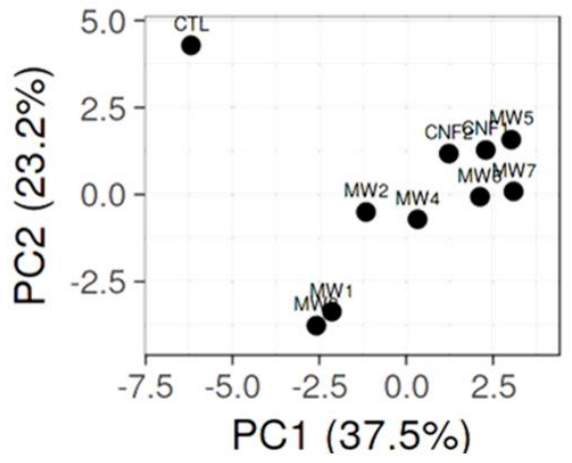

Supplementary Figure 2.3 Principle component analysis results from all characterization (A), L-W-AR binning (B), and means only (C) combined with the four primary in vitro outcomes 


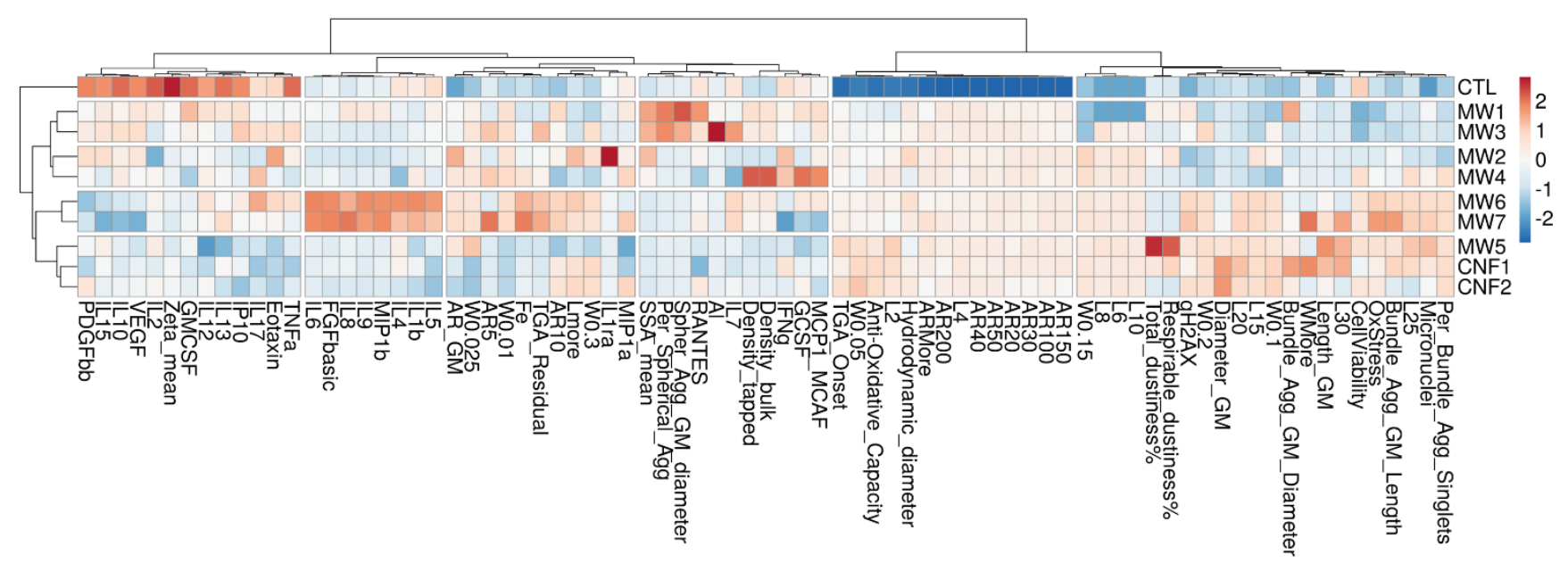

Supplementary Figure 2.4 Hierarchical cluster analysis for all characterization and the four primary in vitro outcomes along with inflammatory protein production 


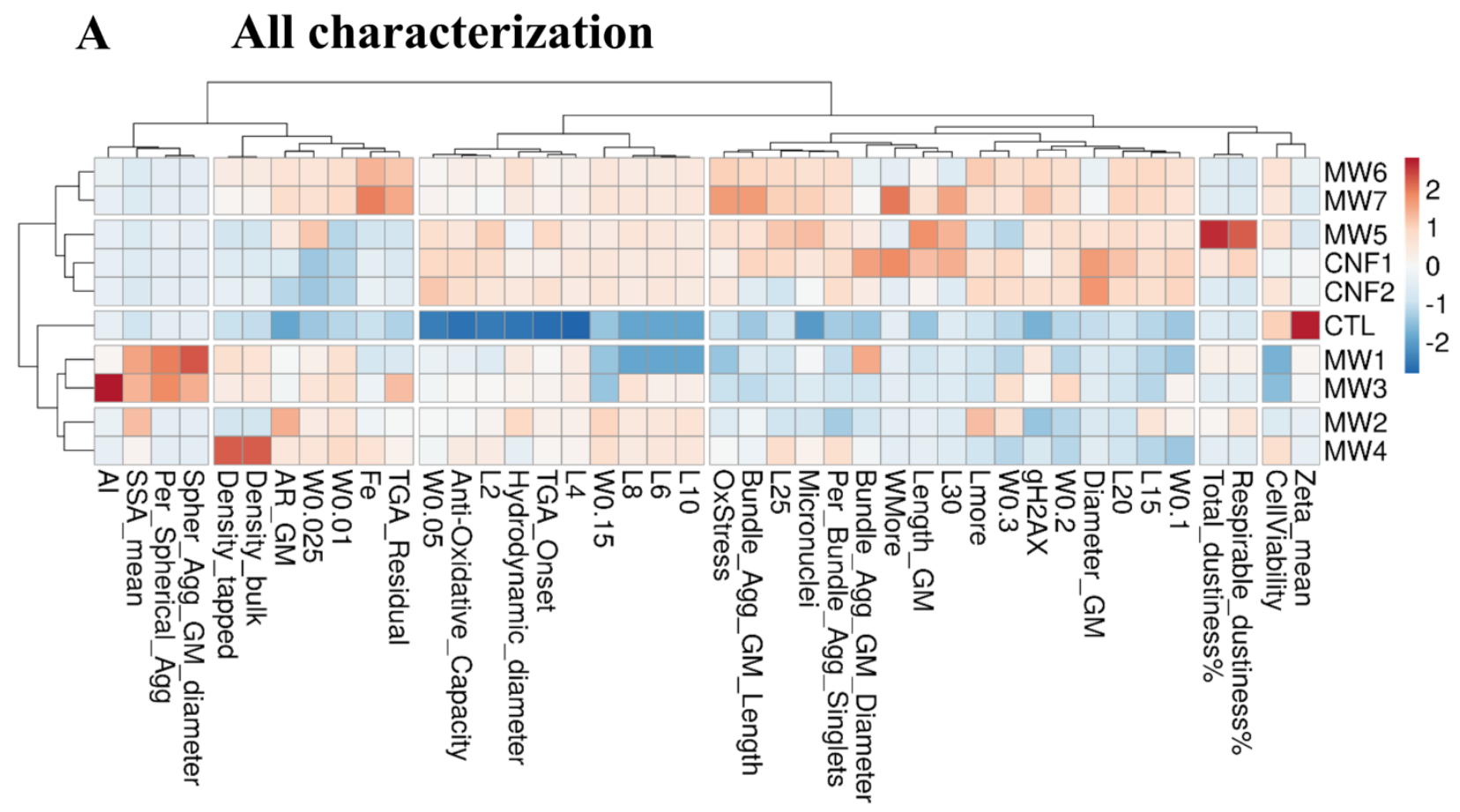

\section{B L-W-AR binning}

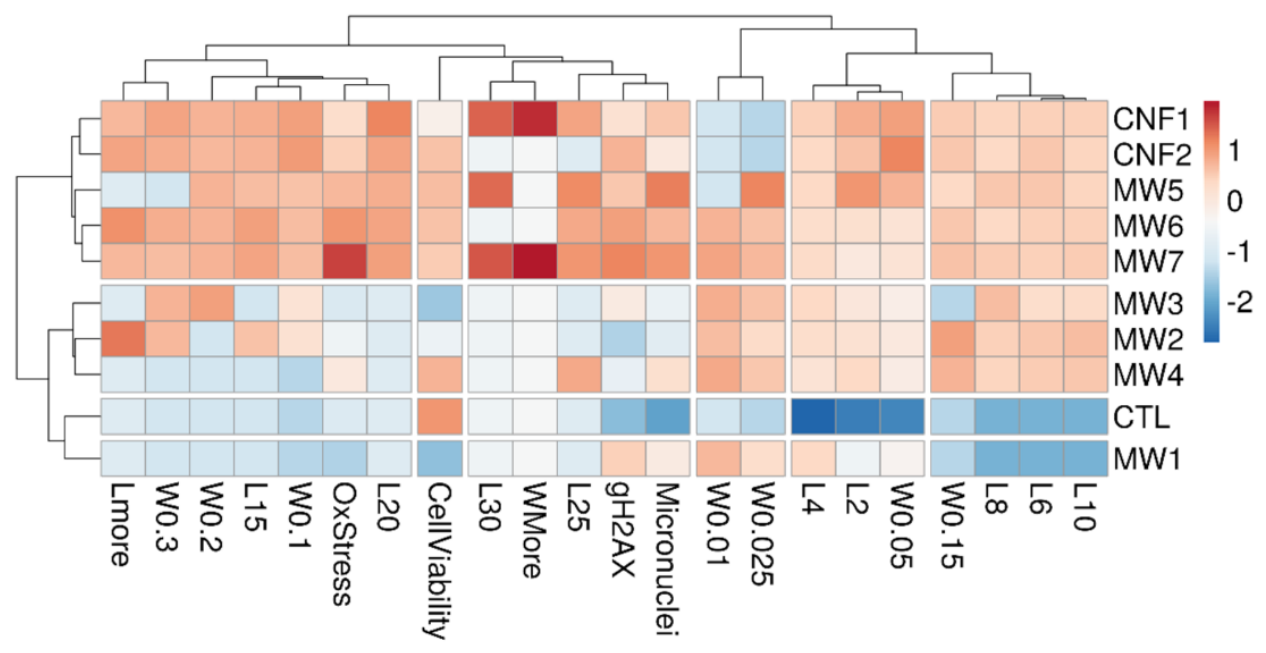

Supplementary Figure 2.5 Hierarchical cluster analysis without aspect ratio inning for all characterization (A) and L-W-AR binning (B) when considering the four primary in vitro epithelial toxicity outcomes 

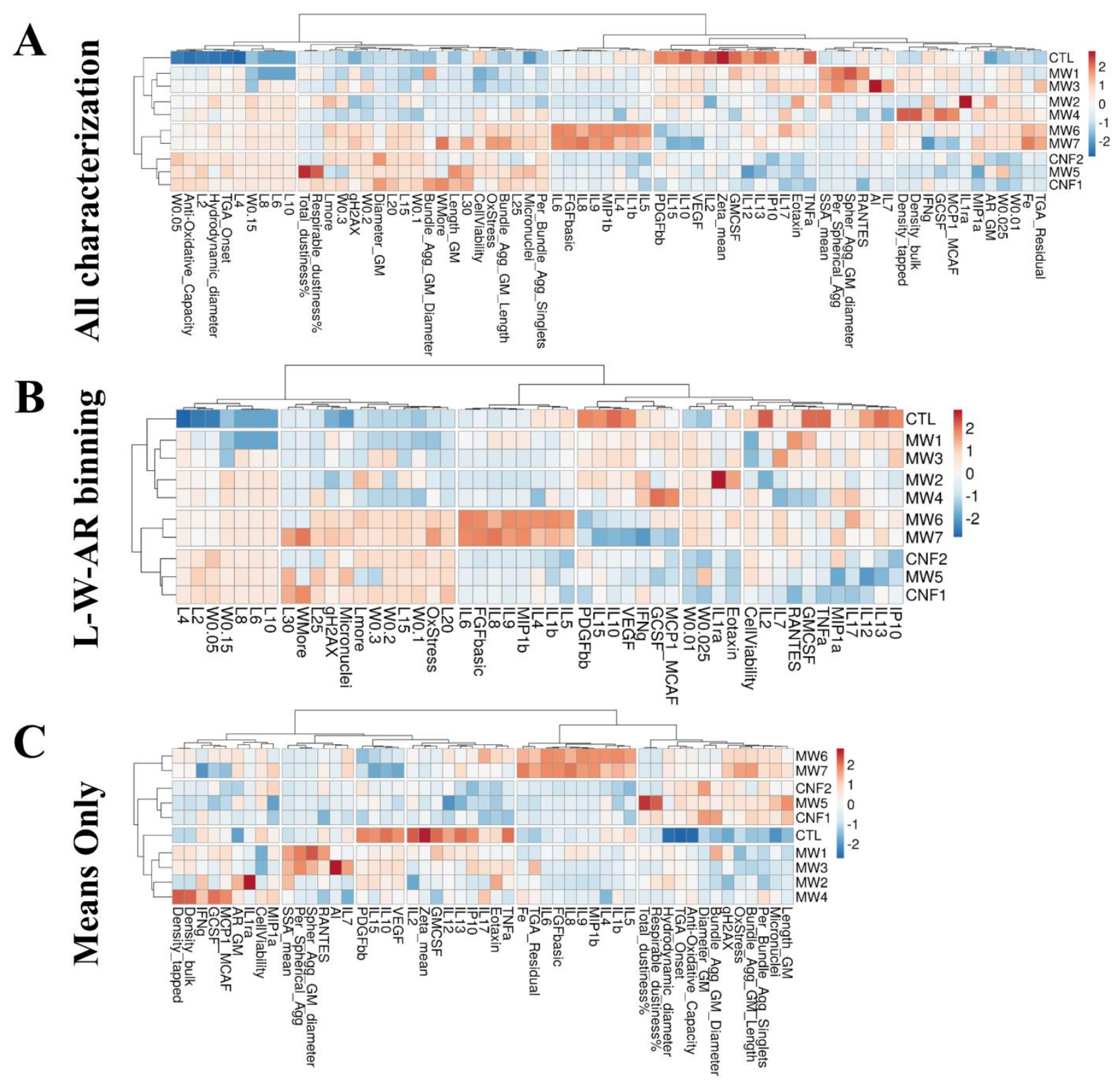

Supplementary Figure 2.6 Hierarchical cluster analysis without aspect ratio inning for all characterization (A), L-W-AR binning (B), and means only (C) when considering the four primary in vitro epithelial toxicity outcomes and protein changes 
$\mathbf{A}$

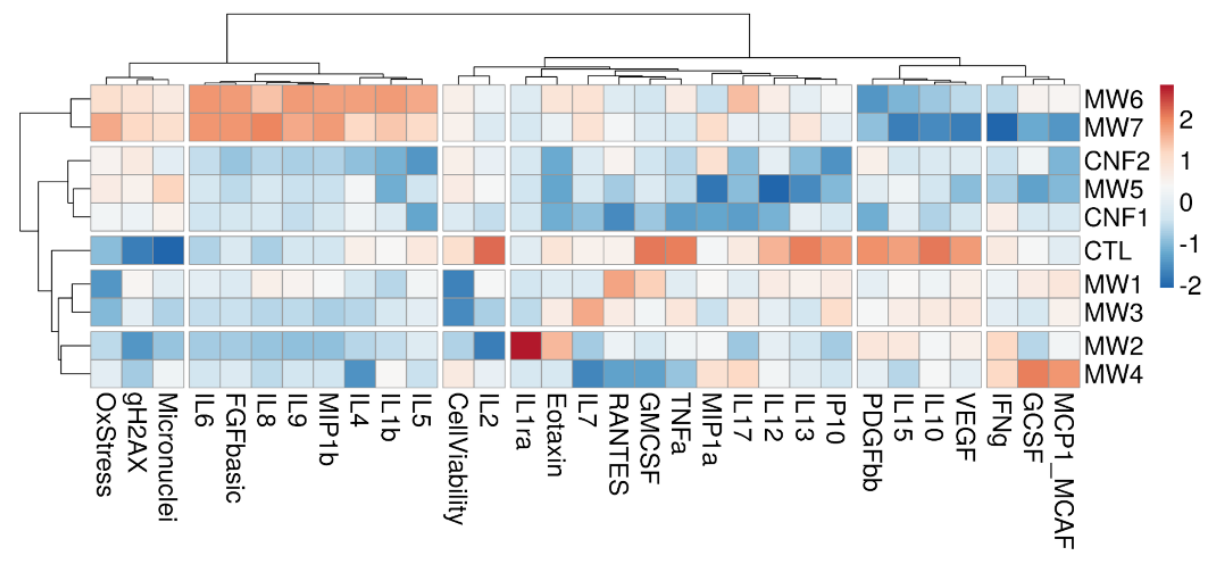

B

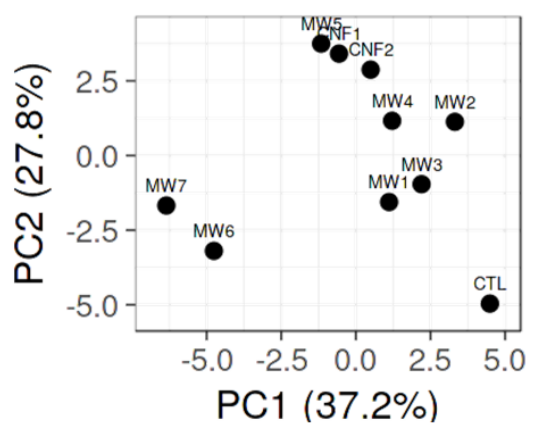

Supplementary Figure 2.7 Hierarchical cluster analysis (A) and principle component analysis (B) without aspect ratio binning for the four primary in vitro epithelial toxicity and protein production only 


\title{
CHAPTER 3:
}

\section{Inflammation and Respective Physicochemical Drivers of the Broad Class of Carbon Nanotubes and Nanofibers Used or Produced in U.S. Facilities.}

\author{
Kelly Fraser ${ }^{1,2}$, Vamsi Kodali ${ }^{1,2}$, Naveena Yanamala ${ }^{1}$, Patti C. Zeidler-Erdely ${ }^{1,2}$, Tracy Eye ${ }^{1}$, John \\ Hubczak $^{1}$, Sarah Foster ${ }^{1}$, Lindsey Bishop ${ }^{1,2}$, Gary Casuccio ${ }^{3}$, Kristin Bunker ${ }^{3}$, Traci L. Lersch ${ }^{3}$, Michael \\ L. Kashon ${ }^{1}$, Sherri Friend ${ }^{1}$, Matthew Dahm ${ }^{4}$, Mary K. Schubauer-Berigan ${ }^{4,5}$, Aaron Erdely ${ }^{1,2}$
}

${ }^{1}$ Health Effect Laboratory Division, National Institute for Occupational Safety and Health, Morgantown, WV; ${ }^{2}$ West Virginia University, Morgantown, WV; ${ }^{3}$ RJ Lee Group, Monroeville, PA; ${ }^{4}$ Division of Field Studies Evaluation, National Institute for Occupational Safety and Health, Cincinnati, OH; ${ }^{5}$ International Agency for Research on Cancer, Lyon, France

Publication in progress 


\subsection{Abstract}

Background: Pulmonary exposure to carbon nanotubes or nanofibers (CNT/F), known to induce inflammation, toxicity, or tumorigenesis, is a concern during production and dry powder handling. CNT/F represent a large class of materials and it is unclear if all confer similar toxicity. Our aim was to simultaneously assess pulmonary inflammation induced by $\mathrm{CNT} / \mathrm{F}$ with variable physicochemical properties obtained from U.S. facilities. Cytotoxicity and inflammation were assessed in mice 1, 7, 28, and $84 \mathrm{~d}$ following oropharyngeal aspiration to 4 or $40 \mu \mathrm{g}$ of one of nine CNT/F (MW \#1-7 and CNF \#12). In complement, inflammasome activation and mechanisms of inflammation were assess in vitro in human THP-1 macrophages, and computational modeling was used to investigate the relationships between physicochemical characteristics and toxicity outcomes.

Results: Lactate dehydrogenase (LDH) activity, a marker of cytotoxicity, was dose-dependently increased in bronchoalveolar lavage fluid (BALF) resolving toward baseline by $84 \mathrm{~d}$ in all groups. In materials with a diameter greater than or equal to $50 \mathrm{~nm}, \mathrm{LDH}$ was persistently increased. Polymorphonuclear cell infiltration (\% PMN), a marker of inflammation, was increased in all materials at $1 \mathrm{~d}$ post-exposure to 40 $\mu \mathrm{g}(<50 \mathrm{~nm}: 31.1 \%, \geq 50 \mathrm{~nm}: 37.1 \%)$. With exposure to materials less than $50 \mathrm{~nm}$, PMN influx mostly resolved by $7 \mathrm{~d}$ while materials greater than or equal to $50 \mathrm{~nm}$ induced persistent inflammation $(7 \mathrm{~d}$ : $<50$ $\mathrm{nm}: 10.5 \%, \geq 50 \mathrm{~nm}: 48.9 \%$ ). For complement, inflammatory gene expression in lung tissue (e.g., $I l-1 b$, Il-6, Ccl22, Cxcl2) and protein levels in BALF (e.g. Il-1b, Il-6, Il-5, Ccl22, Cxcl1), were elevated to a greater extent in materials with a nominal tube diameter greater than or equal to $50 \mathrm{~nm}$ These findings correlate with in vivo findings.

Conclusion: All CNT/F induced pulmonary inflammation, though more severe and more persistent inflammation was associated with materials containing subpopulations of tubes with greater nominal tube diameters and lengths. 


\subsection{Introduction}

Over a decade of research has been competed investigating the potential adverse health effects following respiratory exposure to carbon nanotubes and carbon nanofibers (CNT/F). These studies have consistently demonstrated that $\mathrm{CNT} / \mathrm{F}$ could induce pulmonary inflammation, fibrosis, and more recently genotoxicity and cancer $[9,11,49,51,162,172,173]$. Adverse systemic effects of the immune, nervous, and cardiovascular systems following a pulmonary have been reported[63, 85, 87, 88, 121]. Additionally, $\mathrm{CNT} / \mathrm{F}$ can exit the lung either as small bundles or singlets and deposit in systemic organs $[60,64]$. While a multitude of outcomes have been investigated, minimal correlations between physicochemical characteristics and toxicity outcomes have been elucidated while simultaneously evaluating a broad spectrum of CNT/F. Even fewer studies connect human occupational exposure with laboratory-based experimental approaches. Human exposure-informed design of in vivo and in vitro experimental approaches allows for better extrapolation of potential human health concerns.

Knowledge of human health outcomes of CNT/F exposure are currently limited as the latency for adverse chronic outcomes has not been reached due to the relatively young field of CNT/F large-scale manufacturing. In fact, biomarkers of exposure and effect, especially for inflammation, were minimal and inconsistent [73, 76-79]. More sensitive measures have been necessary to evaluate human exposure [89]. For this reason, studies using in vivo and in vitro modeling systems have become necessary to understand and predict exposure risks. Historically, many of these studies have frequently investigated a single material. The exposure science of CNT/F suggested varying physicochemical characteristics of the broad class of CNT/F $[68,69,133]$.

The International Agency for Research on Cancer (IARC) has only classified Mitsui-7/MWCNT7 as possibly carcinogenic to humans (Group 2B) while also classifying all other CNT/F as having insufficient evidence of carcinogenicity (Group 3) [115]. This lack of evidence regarding the carcinogenicity of CNT/F has sparked the need for more in-depth studies as well as study designs that 
include a sampling of materials with a broader representation of physicochemical characteristics. Furthermore, IARC has issued their 2020-2024 Report of the Advisory Group to Recommend Priorities for the IARC Monographs which suggests that MWCNT are a high priority and should be ready for evaluation within five years [37].

In 2000, and again revisited in 2011, Hanahan and Weinberg established their theory of the hallmarks of cancer, which are the biological processes that are altered during tumor formation [60, 174, 175]. One these hallmarks, inflammation, has been investigated for its role in carcinogenesis. Mitsui-7 is known to induce chronic inflammation, though it is unclear if all CNT/F induce the same inflammatory mechanisms or are equally persistent and potent inflammatory drivers.

Inflammation alone is not the only hallmark of carcinogenicity, and cancer is not the only toxicity outcomes of CNT/F of concern. Understanding the four key toxicity outcomes including genotoxicity, inflammation, histopathology, and extra-pulmonary translocation, and their relationships with one another, is necessary to fully realize the risks associated with respiratory exposure to CNT/F.

The aim of this study was to generate and accurate and effective safety profile of numerous CNT/F of varying physicochemical characteristics in conditions that best represent occupational exposure. Using an extensive in vivo toxicity assessment of nine different materials, seven MWCNTs and two CNF, we investigated the inflammation and cytotoxicity induced by these materials at both acute and sub-chronic post-exposure time points as well as two doses, 4 and $40 \mu \mathrm{g}$. Furthermore, in vitro assessments were completed to further investigate the mechanisms by which these materials induce inflammation and cellular injury. Computational modeling was also incorporated to fully assess the relationships between particle physicochemical characteristics and in vitro and in vivo toxicity assessments. 


\subsection{Results and Discussion}

The nine materials used in this study were selected to represent a broad spectrum of physicochemical characteristics. Seven MWCNT and two CNF (CNT/F), which included the benchmark material, Mitsui-7/MWCNT-7 (MW \#5), were arranged according to their diameter as reported by the production facility and are referred to as MW \#1-7 and CNF \#1-2. The details of this characterization was previously published and can be found in the supplementary data (Supplementary Tables 1-3, Supplementary Figures 1-2) [176]. In the previous study in this series, principle component analysis and hierarchical clustering were used to determine the key role of particle size dimensions as a means of grouping [176]. It was determined that the means of length, diameter, and aspect ratio alone were insufficient for grouping $\mathrm{CNT} / \mathrm{F}$, and a fuller, more detailed understanding of the sub-populations of particle dimensions was necessary. Furthermore, this study investigated the link between these physicochemical characteristics and genotoxicity and found that CNT/F with notable sub-populations of greater nominal tube diameter and length induced more severe genotoxicity than materials with more homogenous populations of smaller diameters and lengths. For the current study, in vivo assessments were evaluated to compare differences in lung injury, lung inflammation, and translocation and which physicochemical characteristics contributed to those outcomes. Further, complementary assessments in vitro addressed comparability to the in vivo setting with the potential elucidation of mechanisms driving those toxicities.

In vivo dosing in the current study has been optimized to correlate to human workplace exposures previously assessed $[68,69,92]$. Previously we have documented the importance of integrated exposure and toxicity assessments for representative laboratory-based toxicity study design [19]. The ability to accurately represent material characteristics from a worker exposure aids in providing the proper context to address potential human health effects. As previously demonstrated for different CNT materials dispersion methods were designed to replicate the agglomeration size and particle dispersion that is 
commonly observed in the personal breathing zone samples taken from facility workers [19]. Supplemental Figure 3 illustrates representative images of personal breathing zone samples paired with images of the same material dispersed in physiologic dosing media used for toxicity evaluations. Additionally, the doses in the current study were selected to represent human exposures. The lower dose was selected due to its correlation to human occupational exposure. This $4 \mu \mathrm{g}$ dose correlates to the cumulative deposited dose of approximately 7.6 years spent working in in a facility at average workplace exposures [164]. The 7.6 years also represents a good approximation of the current state of the workforces in terms of average years working in a CNT/F facility. Previous studies have shown that a higher dose of $40 \mu \mathrm{g}$ was necessary to induce pathological changes, significant pulmonary injury and inflammation, and systemic effects for the reference material, MW \#5/MWCNT-7/Mitsui-7.

\subsubsection{In vivo toxicity assessments}

For evaluation of toxicities in vivo, male C57BL/6J mice were exposed to 4 or $40 \mu \mathrm{g}$ of $\mathrm{CNT} / \mathrm{F}$ or DM via oropharyngeal aspiration. Mice were euthanized at one of four time points: $1,7,28$, and 84 days post-exposure. These exposures were completed in a block design with a total of 12 sets of mice divided into two groups. A schematic demonstrating the dosing timeline can be found in Supplemental Figure 4. Bronchoalveolar lavage was performed on the first group of mice to quantify inflammation using markers such as polymorphonuclear (PMN) cell infiltration, lactate dehydrogenase release, and changes in inflammatory protein levels. In the second group of mice, tissue from the right lung was collected and frozen to evaluate changes in inflammatory gene expression. The entirety of dosing and tissue collection occurred within a 12-month window. Consistency of dosing and data collection across this expansive time window was achieved as later demonstrated within the statistical analysis. 


\subsubsection{Macrophage handling of $\mathrm{CNT} / \mathrm{F}$}

At $7 \mathrm{~d}$ post-exposure, macrophages collected from bronchoalveolar lavage fluid (BALF) were isolated and imaged using scanning electron microscopy (SEM). Representative images are shown in Figure 1. Depending on particle size and agglomeration, macrophage uptake was variable. Macrophages from mice exposed to materials of smaller physical size and agglomerates, such as MW \#1 and \#3, were imaged as intact cells that are presumed to be able to easily engulf smaller particles and agglomerates (Figure 1A, C). While not visible in SEM images, light microscopy images of macrophages from BALF were seen to contain black agglomerates of particle (Supplementary Figure 5). MW \#2 was most commonly present in large agglomerates that are too large for phagocytosis (Supplemental Table 2; Figure 1B). Macrophages can be found adhering to the large agglomerates demonstrating some cellular interactions. MW \#4 is a transition material that has some agglomerates small enough to be phagocytosed, as seen encapsulated in a presumably dying cell as well as in light microscopy images (Figure 1D, Supplementary Figure 5D). Beginning with MW \#5, CNT/F have length populations that becomes difficult for macrophages to phagocytose, as seen in MW \#5 (Figure 1E-I). Some materials have subpopulations that are impossible to phagocytose resulting in the potential for diminished clearance, persistent cellular injury, and increased inflammatory activation. This encumbered particle uptake is further supported by images of BALF macrophages taken using standard light microscopy (Supplementary Figure 5E-I).

Mercer et al. in 2011 commented on the macrophage uptake of MWCNTs compared to singlewalled carbon nanotubes (SWCNTs) [9]. As SWCNTs only have one wall, these tubes are drastically smaller in diameter compared to many MWCNTs. The findings in the 2011 study suggest that there is a lower limit regarding macrophage uptake. It can be hypothesized that SWCNTs are too small to engage macrophage recognition and response in comparison to MWCNTS resulting in only $10 \%$ macrophage uptake of SWCNTs, vastly reduced compared to 70\% of MWCNT uptake. Of further note, in 2013 Mercer 
et al. also commented that MWCNTs dosed via bolus aspiration compared to inhalation had greater macrophage uptake [10]. As bolus dosing results in more heavily agglomerated particles, these findings suggest that agglomerate size will also influence macrophage response. Our findings support that macrophage uptake occurs when particles are large enough to engage macrophage recognition but are not too large that uptake cannot occur due to the size constraints of the cell.

\subsubsection{Pulmonary Response}

\subsubsection{One day post-exposure}

Lactate dehydrogenase ( $\mathrm{LDH})$ is a cytosolic enzyme that can be measured in BALF to quantify cytotoxicity. CNT/F were suspected to induce cytotoxicity at this time point by piercing and damaging cell membranes. At 1 d post exposure, LDH was increased dose dependently (Supplementary Figure 6A). This was expected following bolus dose administration. MW \#5-7 and CNF \#1-2 low dose exposures also induced a significant increase in LDH suggesting more cytotoxicity at lower deposited doses as the physical dimensions increase in size.

As an additional measurement of inflammation, inflammatory cell infiltration was quantified. Inhalation of $\mathrm{CNF} / \mathrm{F}$ can result in injury to the epithelium and inflammasome activation of responding alveolar macrophages, leading to cytokine and chemokine production and the subsequent infiltration of inflammatory cells (Supplementary Figure 6B; Supplemental Table 4). Polymorphonuclear cells (PMN), specifically neutrophils, were increased in all treatment groups at $1 \mathrm{~d}$ post-exposure. Eosinophils, another type of PMN, were significantly increased in all groups except low doses of MW \#1-3.

The relative fold change of mRNA expression for several genes were measured and represented as a heat map compared to DM (Supplementary Figure 7; Supplementary Table 5). The specific panel of genes measured included: $I l-1 b, I l-6, C c l 2, C c l 22, C x c l 2, S p p 1, M t 1, M t 2$, and Hmox1. Il-6 and Il-1b are acute inflammatory markers, with $I l-1 b$ associated with activation of the inflammasome. Activation of 
these genes at early time points indicate cellular injury and increased inflammation acutely post-exposure. Ccl2 is a monocyte chemoattractant to signal for the infiltration of inflammatory cells to the site of injury. Ccl22 is a macrophage derived pro-inflammatory cytokine implicated in the promotion of persistent inflammation and a biomarker linking inflammation and pathology of CNT/F exposure [155, 156]. Cxcl2 is a potent neutrophil chemoattractant. Increases in these genes indicate that pulmonary inflammation was persistent through later time points. Sppl, or osteopontin, is a macrophage chemoattractant that is implicated in histopathological changes specifically granuloma formation. $M t 1, M t 2$, and Hmoxl are stress response genes that increase in response to many things including metal exposure, such as $\mathrm{CNT} / \mathrm{F}$ catalysts, and oxidative stress.

At $1 \mathrm{~d}$ post exposure to high doses of CNT/F, all treatment groups had significantly increased $\mathrm{Il}-6$ and $C c l 2$ expression following high dose exposure. This was expected given the bolus exposure. $I l-1 \beta$ was significantly increased following exposure to high doses of MW \#4-7 and CNF \#1. Ccl22 was significantly increased following high dose exposure to all materials except MW \#2. Cxcl2 was increased following exposure to MW \#4, \#5, and \#7 as well as CNF \#1 and \#2. At $1 \mathrm{~d}$ post-exposure to low doses of CNT/F, Il-6, $C c l 2$, and $C x c l 2$ were significantly increased in all groups. Mtl was increased with exposure to MW \#5 and 7, and Mt2 was increased with exposure to all but MW \#1, 3, and CNF \#2. Hmoxl had was significantly increased following exposure to CNF \#1.

\subsubsection{Seven days post-exposure}

At 7 d post exposure, a significant increase in LDH was seen in all materials following high dose exposure with greater severity in MW \#5-7, and CNF \#1 (Figure 2A). MW \#5-7 and CNF \#1-2 also induced a significant increase in LDH in the low dose. Inflammatory cell infiltration was significantly increased as seen in Figure 2B. Macrophages were not significantly increased, although neutrophils were increased in all exposures and doses. Interestingly, eosinophils also were significantly increased in all 
CNT/F except MW \#2 high dose exposures and low dose exposures of MWCNT \#5-7, and CNF \#1-2. Eosinophils made up over 30\% of BALF harvested cells following MW \#5 high dose exposure, and over $53 \%$ of MW \#6 high dose exposure. This response is also often a greater response than seen at $1 \mathrm{~d}$ postexposure, particularly induced by particles of larger length and diameter. For Mitsui-7 (MW \#5) similar patterns of inflammation have been previously reported [51]. Previous studies have also linked MWCNT exposure to an increase in eosinophil infiltration which may be linked to IgE mediated airway allergy responses $[56,83,177]$.

Changes in proteins that regulate inflammation were assessed. Rodent MAP 4.0 analysis (Ampersand Biosciences, Saranac Lake NY) of 42 proteins was completed from BALF. At 7 d postexposure, several proteins associated with inflammation, wound response, and remodeling of the extracellular matrix were elevated including (Figure 2C). This elevation was seen in almost all high dose treatment groups, but the greatest increase was seen in materials of greater nominal tube diameter and length, primarily MW \#5-7, and CNF \#1. These materials also induced significant increases in proteins following low dose exposures (Figure 2C). MW \#2 induced fewer changes in protein concentrations than other materials as a general trend, this may specifically due to the highly entangled nature of the material contributing to reduced alveolar deposition than other CNT/F [19]. Of special note, increases in eotaxin verifies the increase in eosinophil infiltration observed in cell counts and differentials.

At $7 \mathrm{~d}$ post-exposure, gene expression was measured following exposure to high doses of MWCNT/CNF (Supplementary Figure 6). Il-6 was significantly increased after exposure to all materials except MW \#4. Il-1b expression was significantly increased following exposure to MW \# 2, 5-7, and CNF $\# 1$ and 2. These results further support BALF protein quantifications. Additionally, $C c l 2$, Sppl, and Cxcl2 were increased in all groups, while $C c l 22$ was increased after exposure to MW \#3-7, and CNF \#1-2. These findings further support the previously quantified increase in neutrophil infiltration. Furthermore, $M t l$ was increased following exposure to MW \#4-7, CNF \#1 and 2. Mt2 was increased in MW \#5, 7, and CNF \#1- 
2, while Hmoxl was increased in MW \#5, 7, and CNF \#1 and 2 exposure indicating some oxidative stress may be occurring following exposure to materials of greater diameter.

\subsubsection{Twenty-eight days post-exposure}

Persistent inflammation was observed at $28 \mathrm{~d}$ post-exposure to several materials. MW \#5-7 and CNF \#1-2 had increased LDH following high dose exposures (Figure 3A). Total cell counts were not significantly increased, though inflammatory cell infiltration of specific cell types remained elevated in some treatment groups (Figure 3B). Macrophage infiltration was significantly increased in MW \#1 and \#7 high dose exposed mice. Neutrophil infiltration was increased following exposure to all materials at both doses. Eosinophil infiltration resolved in most groups except high dose exposure to MW \#5-7 and CNF \#1-2. At 28 d post-exposure, several inflammatory proteins were still increased in groups exposed to high doses of particles with minimal changes in proteins following exposure to MW \#2 and \#4 (Figure 3C). MDC, TNFa, Il-12-p40, and CCl6 were also increased following low dose exposure to MW \#7, and MIP-1 $\gamma$ was increased following low dose exposure to MW \#5-7.

Gene expression was also assessed following high dose exposures at $28 \mathrm{~d}$ post-exposure (Supplementary Figure 6). Il-6, Ccl22, and $C x c l 2$ expression was persistently increased in all groups except MW \#2. Spplwas increased in all groups. Ccl2 was increased following exposure to all except MW \#2 and 5, and Il-1 $\beta$ had fully resolved to baseline for all except MW \#1 and 6. Mt1 was persistently increased in materials of larger diameter (MW \#4,-7, CNF \#1-2), while Mt2 was still increased in MW \#5 and 6. Hmoxl was also increased in all except M\#1, 3, and 4.

\subsubsection{Eighty-four days post-exposure}

By 84 d post-exposure, LDH in all low dose exposures and several high dose exposures had resolved. However, high doses of MW \#5-7, and CNF \#1 still had increased LDH (Figure 4A). While total 
cell counts and macrophage counts were not significantly increased, high doses of MW \#1, 5-7, and CNF \#1-2 still had increased neutrophil infiltration (Figure 4B). All other treatment groups had completely resolved inflammatory cell infiltration. Proteins from BALF were also assessed at $84 \mathrm{~d}$ post-exposure, and some significant changes from DM persisted through later time points. IL-6, MMP-9, MDC, MIP-1 $\beta$, MIP-1 $\gamma$, TNF $\alpha$, KC/GRO, Il-12-p40, CCl6, IP-10, MCP-1, IL-28, VCAM1, Eotaxin, GM-CSF, IL-17a, and CRP remained elevated, particularly in exposures to MW \#1, 5-7, and CNF \#1 (Figure 4C).

Most gene expression changes had resolved with a few exceptions of persistent activation (Supplementary Figure 6). In high dose exposures, Il-6 and Ccl2, despite being markers of acute inflammation, were still increased following MW\#1, 3, and 5 exposures. $I l-1 b$ was resolved in all groups. Ccl22 was still increased in most groups except MW \#2-4, and Cxcl2 was increased in most groups excluding MW \# 2 and 4. The persistent activation of these markers support the continued infiltration of inflammatory cells from BALF assessments. Sppl was increased in most groups except MW \# 2-3. Markers of oxidative stress including $M t 1, M t 2$, and Hmoxl had resolved to baseline by 84 d post-exposure except for $M t 2$ following CNF \#2 exposure which had a significant increase in expression.

In low dose exposures, $I l-6$, and $I l-1 b$ had returned to baseline in all groups. MW \#6 still induced a significant increase in Spp1, while all except CNF \#2 induced significant increases in Cxcl2, Ccl2 and Ccl22 was still significantly increased CNF \#1 and MW \#7, respectively.

\subsubsection{Inflammation summary}

The general inflammatory response pattern was not unexpected due to findings previously published regarding $\mathrm{CNT} / \mathrm{F}$ inflammation. The toxicity outcomes having representation in all $\mathrm{CNT} / \mathrm{F}$ at 1 d post exposure was expected following a bolus administration. What was noted in this study was the persistence and increased magnitude of lung injury and inflammation for materials with larger physical dimensions at $7 \mathrm{~d}$ post-exposure. The results were consistent with Porter et al. in 2010, which evaluated 
MW \#5, in that $7 \mathrm{~d}$ post-exposure resulted in greater lung injury and inflammation compared to day 1 for a $40 \mu \mathrm{g}$ dose. Resolution of inflammation at $28 \mathrm{~d}$ and $84 \mathrm{~d}$ post-exposure measured in this study was consistent with previous evaluation of various CNT [51, 82, 124].

As a general trend, materials of greater nominal tube diameter had more severe and more persistent cytotoxicity and inflammatory cell infiltration than materials of smaller diameter, though almost all materials induced cellular injury that persisted through $28 \mathrm{~d}$ post-exposure. These larger materials also were more likely to induce the infiltration of eosinophils in addition to neutrophils and macrophages. Changes in inflammation regulating proteins from BALF and their corresponding changes in relative mRNA expression also follows a similar correlation to nominal tube diameter.

While particle size is frequently noted to be related to the observed inflammatory response, other studies have noted the potential role of other physicochemical characteristics as drivers of toxicity outcomes. In addition to noting the role of particle dimensions, a series of studies from the National Research Centre for the Working Environment (Denmark) noted that BET surface area as well as diameter and length significantly correlated with inflammatory cell infiltration as well as genotoxicity measurements. Additionally, particle agglomeration and rigidity has also been considered as an influential factor for CNT toxicity. Previous studies comparing the toxicity of CNT of various rigidity and agglomeration patterns [56]. This study found that rigid MWCNT were more inflammatory and cytotoxic than less rigid materials. These findings correlate with the conclusions of this study in which materials with more classic fiber characteristics were more toxic than smaller agglomerated materials.

\subsubsection{HCA - PCA for inflammation}

To understand the relationship and grouping of CNT/F by inflammation the outcomes of cytotoxicity and cellular inflammation at all days and time points were evaluated (Supplemental Figure 8). Clearly the most significant responders were MW \#6, \#7, and CNF \#1 at $7 \mathrm{~d}$ post-exposure with an 
exposure to $40 \mu \mathrm{g}$. Interestingly, the same materials were evident with only $4 \mu \mathrm{g}$ exposure. Given the more realistic nature of the $4 \mu \mathrm{g}$ deposited dose in terms of human relevance, that exposure was utilized for modeling. Given the clear evidence of persistent inflammation at $7 \mathrm{~d}$ that time point was chosen as a representative discriminator to distinguish effects of the various CNT/F. Avoidance of a non-specific bolus response to a deposited dose $1 \mathrm{~d}$ post-exposure and determining lung injury and inflammation prior to resolution also made $7 \mathrm{~d}$ post-exposure the desired time point.

When modeling lung injury and cellular influx alone, MW \#6, \#7, and CNF \#1 were clearly the materials generating the greatest toxicity at relevant exposure levels (Figure 5). The other materials were not without effect (Figure 2), but not as marked in the response at $7 \mathrm{~d}$ post-exposure following a $4 \mu \mathrm{g}$ deposited dose. Previously, when analyzing physicochemical characteristics alone and in conjunction with genotoxicity, it was observed that binning of physical dimensions, either alone or in addition to traditional characteristics means, provided greater resolution for grouping materials and determining which characteristic predicted an outcome (Fraser 2020). An additional measure of two-dimensional sizing, an exposure assessment driven additional characterization (Dahm 2018, Fraser 2020) was a good predictor of toxicity outcomes but not typically included as part of general characterization. When including all characterization parameters, mean, physical dimension binning, and two-dimensional sizing, MW \#6, MW \#7, and CNF \#1 were in the greater toxicity group along with MW \#5 and CNF \#2 (Figure 6). The physicochemical characteristics driving the greater severity in lung injury and cell influx were greater physical diameters and length along with the lengths of bundled agglomerates. Analysis by binned physical dimensions alone highlighted MW \#7 and CNF \#1 as particularly driving an adverse response with greater length and width parameters associated with toxicity outcomes (Supplemental Figure 9A). Using means only for physicochemical characteristics did not group any of the traditional measures with outcomes (Supplemental Figure 9B). The only close grouped characteristic was bundled agglomerate mean length, part of our added two-dimensional sizing. 
Proteins were evaluated separately given the large number analyzed. Analysis of protein changes alone also had MW \#6, MW \#7, and CNF \#1 as generally more toxic as would be predicted by the other injury and inflammation markers (Supplemental Figure 10A). MW \#5 was also included in the group exhibiting an increased toxicity response. Similar separations were made when adding the physicochemical characteristics, especially when binning of physical dimensions were considered (Supplemental Figure 10B).

\subsubsection{In vitro screening}

The last two decades of research into CNT/F toxicity reveled mechanisms and signaling pathways modulated by $\mathrm{CNT} / \mathrm{F}$ exposure. Some of the key events modulated in macrophages include cell viability,

membrane damage, nuclear factor $-\kappa \mathrm{B}(\mathrm{NF}-\kappa \mathrm{B})$-based inflammation, NLRP3-based inflammasome activation, pyroptosis, and alteration in innate immune function. Using differentiated THP-1 macrophages, a traditional cell type used to mimic pulmonary macrophage function, parallel in vitro assessments were performed to screen the nine $\mathrm{CNT} / \mathrm{F}$ for their potency with respect to various biomarkers and endpoints and explore mechanisms of inflammatory modulation.

\subsubsection{Cell viability and membrane damage}

Change in cell viability due to exposure of the nine CNT/F was assessed by challenging the differentiated THP-1 macrophages with the nine CNT/F for $24 \mathrm{~h}$ at $0,1.87 \mu \mathrm{g} / \mathrm{ml}, 3.75 \mu \mathrm{g} / \mathrm{ml}, 7.5 \mu \mathrm{g} / \mathrm{ml}$, $15 \mu \mathrm{g} / \mathrm{ml}, 30 \mu \mathrm{g} / \mathrm{ml}, 60 \mu \mathrm{g} / \mathrm{ml}$, and $120 \mu \mathrm{g} / \mathrm{ml}$ (Figure 8A). These concentrations in terms of surface area corresponds to $0,0.58 \mu \mathrm{g} / \mathrm{cm}^{2}, 1.17 \mu \mathrm{g} / \mathrm{cm}^{2}, 2.34 \mu \mathrm{g} / \mathrm{cm}^{2}, 4.68 \mu \mathrm{g} / \mathrm{cm}^{2}, 9.37 \mu \mathrm{g} / \mathrm{cm}^{2}, 18.75 \mu \mathrm{g} / \mathrm{cm}^{2}$, and $37.5 \mu \mathrm{g} / \mathrm{cm}^{2}$ respectively. Change in cell viability due to $\mathrm{CNT} / \mathrm{F}$ exposure was evaluated by measuring bioreduction of tetrazolium salt WST-1 by CNT/F exposed cells and comparing it with healthy control cells with no exposure. MW \#6 and MW \#7 were the most potent among the screened CNT/F. Compared 
to control cells a concentration of $3.75 \mu \mathrm{g} / \mathrm{ml}$ of either of these CNT/F was sufficient to significantly reduce the viability of the cells. MW \#1, \#2, and \#4 exposure caused decrease in viability at only the highest dose tested of $120 \mu \mathrm{g} / \mathrm{ml}$. CNF \#1-2 and MW \#3 caused decrease in viability from a concentration of $30 \mu \mathrm{g} / \mathrm{ml}$. MW \#5 started effecting the viability after $15 \mu \mathrm{g} / \mathrm{ml}$. With exception to $\mathrm{MW} \# 3$, the general trend showed that CNT/F with increasing physical dimensions caused increased toxicity.

Membrane damage was assessed by exposing cells to the nine $\mathrm{CNT} / \mathrm{F}$ at the eight concentrations between $0-120 \mu \mathrm{g} / \mathrm{ml}\left(0-37.5 \mu \mathrm{g} / \mathrm{cm}^{2}\right)$ for $24 \mathrm{~h}$ and measuring the released lactate dehydrogenase (LDH) from cells with a damaged membrane (Figure 8B). For most CNT/F a dose-dependent increase in membrane damage was measured. Similar to cell viability, CNT/F with larger physical dimensions caused increased membrane damage. MW \#5 was the most potent CNT/F in the screened group and caused a significant increase in toxicity at the lowest test concentration of $1.87 \mu \mathrm{g} / \mathrm{ml}\left(0.58 \mu \mathrm{g} / \mathrm{cm}^{2}\right)$. Compared to the WST-1 consumption assay, membrane damage was much more sensitive in resolving CNT/F induced toxicity. The lowest concentrations at which the nine CNT/F caused membrane damage ranged from 1.87 - $30 \mu \mathrm{g} / \mathrm{ml}\left(0.58-9.37 \mu \mathrm{g} / \mathrm{cm}^{2}\right)$. MW \#2 did not cause any membrane damage even at the highest concentration tested.

\subsubsection{Nuclear factor $-\kappa B(N F \kappa B)$ activation}

$\mathrm{NF} \kappa \mathrm{B}$ activation after exposure to the nine $\mathrm{CNT} / \mathrm{F}$ was monitored by using $\mathrm{NF} \kappa \mathrm{B}$ secreted embryonic alkaline phosphatase (SEAP) Reporter THP-1 cells. Cells were exposed to the CNT/F at the 8 concentrations between $0-120 \mu \mathrm{g} / \mathrm{ml}\left(0-37.5 \mu \mathrm{g} / \mathrm{cm}^{2}\right)$ for $12 \mathrm{~h}$ and the SEAP was quantified to determine NFkB activation (Figure $8 \mathrm{C}$ ). Within the dose tested, exposure to all the nine CNT/F caused a dosedependent increase in activation of $\mathrm{NF} \kappa \mathrm{B}$ signal transduction pathway. The lowest concentration at which there was a significant change in NFאB activation ranged between $3.75-60 \mu \mathrm{g} / \mathrm{ml}\left(1.17-18.75 \mu \mathrm{g} / \mathrm{cm}^{2}\right)$ for the nine CNT/F. At the highest dose evaluated there was $\sim 5$-fold change in NFkB activation by MW 
\#4 and \#6. Among the screened CNT/F, MW \#4 and CNF \#1 were the most potent and caused significant change at $3.75 \mu \mathrm{g} / \mathrm{ml}\left(1.17 \mu \mathrm{g} / \mathrm{cm}^{2}\right)$ while MW\#5 was the least potent and caused significant change at 60 $\mu \mathrm{g} / \mathrm{ml}\left(18.75 \mu \mathrm{g} / \mathrm{cm}^{2}\right)$.

\subsubsection{NLRP3 inflammasome activation and pyroptosis}

Previous structure activity relationship studies showed that high aspect ratio materials like asbestos and CNT induced NLRP3 inflammasome activation and pyroptosis. We measured IL-1 $\beta$ (Figure 9A) and IL-18 (Figure 9B) released by differentiated and primed THP-1 cells as a proxy biomarker measure for NLRP3 inflammasome activation. Using an activity-based probe for caspase-1, we screened for active caspase-1 as a marker for pyroptosis (Figure 9C). Compared to control cells, exposure to the nine CNT/F caused $\sim 1$-3-fold change in IL-1 $\beta$ release and $\sim 2$-fold change in IL-18 release. MW\#5-7 and CNF \#1

caused significant increase in both IL-1 $\beta$ and IL-18 secretion and MW \#2, 3, 4 and CNF\#2 significantly altered IL1 $\beta$ production but not IL-18. Active caspase-1 released due to NLRP3 inflammasome activation is known to cleave the inactive cytoplasmic precursor pro-IL1 $\beta$ and pro-IL18 converting it into a bioactive and mature IL-1 $\beta$ and IL-18. Caspase 1 is a cysteine-rich protease that is known to cleave key cellular substrates leading to pyroptosis or programed cell death. Caspase 1 was measured as an additional marker for NLRP3 inflammasome activation and as a marker for pyroptosis. Active Caspase-1 induced due to CNT/F exposure was assessed by flow cytometry using the fluorescent inhibitor probe FAM-YVADFMK. All the nine CNT/F screened caused induction of active caspase-1 and compared to control cells with MW \#5-7 being potent inducers.

\subsubsection{Alteration in phagocytic capacity}

Immune dysfunction due to particle exposure has been hypothesized as a probable cause for increase in respiratory infections after particulate exposure [178]. NLRP3 inflammasome activation 
orchestrates multiple innate and adaptive immune responses in infection and auto-inflammatory disorders. To screen if CNT/F have a potential to alter innate immune function, THP-1 cells were exposed to 15 $\mu \mathrm{g} / \mathrm{ml}\left(4.68 \mu \mathrm{g} / \mathrm{cm}^{2}\right)$ of various CNT/F for $24 \mathrm{~h}$. Change in phagocytic capacity due to CNT/F exposure was quantified by challenging the exposed cells to green fluorescent protein Escherichia coli (GFP E.coli) for $2 \mathrm{~h}$ at a multiplicity of infection (MOI) of 25. Exposure to all the CNT/F test subjects except MW \#2 and \#3 reduced the phagocytic capacity of the macrophages. The rank order in potency of the CNT/F for reducing macrophage phagocytic capacity was greater for MW \#5-7 and CNF \#1-2 compared to MW \#14. Within the CNT/F test group, the reduction in phagocytic capacity ranged between no change to $64 \%$ of the normal phagocytic capacity.

\subsubsection{HCA - PCA for in vitro}

Computational modeling of all in vitro outcomes grouped CNT/F into two main groups, MW \#14 and MW \#5-7 with CNF \#1-2 (Figure 10A). Control cells grouped with MW \#1-4 indicating attenuated toxicity in comparison to other CNT/F. The grouping remained the same no matter the approach of analyzing with physicochemical characteristics although the binning of physical dimensions offered greater resolution in terms defining effect. The evaluation using all characteristics indicated consistency of inflammasome induction as IL-1 $\beta$, IL-18, and caspase-1 all grouped together (Figure 10B). Cellular cytotoxicity, measured by LDH release, also grouped with inflammasome indicators. Larger lengths and diameters, along with agglomerate characteristics induced greater inflammasome induction and cellular cytotoxicity. Cell viability, $\mathrm{NF \kappa B}$, and phagocytic capacity grouped with smaller widths, specific surface area, and material density. The cellular responses of THP-1 cells (Figure 10B) grouped CNT/F the same as the in vivo responses of inflammation (Figure 6). These results infer the submerged cell culture using THP-1 cells were sufficient to group CNT/F in terms of inflammation and injury. 


\subsubsection{Summary}

It is quite clear there is a broad variance within the class of CNT/F and within a given sample. Materials that were by majority small in length and diameter induced the least amount of inflammation and were quicker to resolve over time. Materials that were larger in diameter and length, even if only a small proportion of nominal tubes fit that criteria, induced greater and more sustained lung injury and inflammatory response. This is likely a direct reflection on the innate immune response handling of materials as the smaller materials were within the dimensions for routine macrophage accumulation. More inflammatory CNT/F were often larger than resident alveolar macrophages and quite commonly seen protruding cellular membranes.

Submerged monocultures of THP-1 cells are routinely used for in vitro particle toxicity studies and accurately reflected in vivo outcomes of CNT/F inflammation and injury. These results, obtained from a simultaneous evaluation of the broad class of CNT/F both in vivo and in vitro, will allow for a rapid predictive outcome of inflammation for $\mathrm{CNT} / \mathrm{F}$.

\subsection{Conclusions}

- All CNT/F have the potential to induce inflammation. Resolution of inflammation occurred with all CNT/F. Materials with populations of CNT/F having larger physical diameters and lengths, contributing to variances in how the materials agglomerate, comparatively had a more persistent and greater magnitude of inflammation.

- The population of larger diameter and length materials only needed to be a small proportion of the CNT/F material to induced greater toxicity.

- Differentiated submerged cultures of THP-1 cells equally grouped CNT/F as in vivo studies in terms of inflammation parameters assessed. 
- Computational modeling using the binned physical dimensions and two-dimensional sizing offered far superior resolution for grouping and determining which physicochemical characteristics were factors for greater inflammation.

\subsection{Materials and Methods}

\subsubsection{Materials}

Multi-walled carbon nanotubes and nanofibers used in this study were as-produced materials provided by six different U. S. facilities. These materials correspond to human exposure assessments previously reported $[69,92]$. One material, MW \#5 is used as reference material in this study as it has been extensively characterized and its toxic effects are well understood.

\subsubsection{In vivo study design}

For the in vivo portion of the study, male C57BL/6J mice ages eight to ten weeks were exposed by oropharyngeal aspiration to either vehicle (physiologic dosing medium; DM), one of seven multi-walled carbon nanotubes (MW \#1, MW \#2, MW \#3, MW \#4, MW \#5, MW \#6, or MW \#7), or one of two carbon nanofibers (CNF \#1 or CNF \#2). Two doses were employed: Low $(4 \mu \mathrm{g})$ or High $(40 \mu \mathrm{g})$. The low dose was selected to best represent current relevant human exposure levels, while the high dose was selected as to induce histopathological changes as previous studies have reported $[9,164]$. Mice were euthanized at $1,7,28$, and $84 \mathrm{~d}$ post-exposure to assess pulmonary inflammation and cytotoxicity.

\subsubsection{Animals}

Male C57BL/6J pathogen-free mice weighing 20-25g were obtained from Jackson Laboratories (Bar Harbor, ME). All mice were housed in the Association for Assessment and Accreditation of Laboratory Animal Care (AAALAC)-accredited NIOSH animal facility and afforded food and tap water 
ad libitum in ventilated cages on autoclaved hardwood chip bedding and an environment of controlled humidity, temperature, and 12:12 light/dark cycles. Animals were allowed to acclimate for at least seven days prior to use in any experiments. Animal care and use procedures were conducted in accordance with the "PHS Policy on Humane Care and Use of Laboratory Animals" and the "Guide for the Care and Use of Laboratory Animals" (2011). The procedures utilized in this study were approved by the National Institute for Occupational Safety and Health Institutional Animal Care and Use Committee.

\subsubsection{Facility representative material preparation and in vivo dosing}

Personal Breathing Zone (PBZ): Images from personal breathing zone samples were generously provided by Mathew Dahm for the comparison of in vivo dosing to relevant human occupational exposures. PBZ samples were collected as previously reported $[69,92]$. These samples were collected from employees working in primary CNT/F manufacturing facilities and were imaged using transmission electron microscopy also as previously reported. PBZ images demonstrate particle appearance, agglomeration, and morphology in human occupation exposures. Images from PBZs taken during particle production are paired with respective in vivo dosing images as available.

Material Preparation: For in vivo exposure, dosing media was prepared fresh prior to dosing and contained mouse serum albumin $(0.6 \mathrm{mg} / \mathrm{ml})$ and 1,2-dipalmitoyl-sn-glycero-3-phosphocholine (DPPC; $0.01 \mathrm{mg} / \mathrm{ml}$ ) prepared in United States Pharmacopeia (USP) grade-phosphate buffered saline (PBS) without calcium and magnesium. Samples were sonicated for $5 \mathrm{~min}$ at the highest setting using an external sonicator (Hielscher Ultrasound Technology) and then for 5 min using a Branson Sonifier 450 probe sonicator set to the lowest setting (10\% duty cycle; output control of 1$)$. These settings and times were determined to best replicate collected PBZ samples as a representation of human exposures.

Oropharyngeal Aspiration: Mice were dosed according to the well-established protocols previously described [179]. In brief, mice were anesthetized using isoflurane. Mice were suspended by their upper 
incisors against a board and the tongue held to prevent swallowing. Either dosing media or particle dispersed in dosing media was pipetted into the throat and aspirated. Particle in dosing media was prepared that each 4 or $40 \mu \mathrm{g}$ dose equaled $50 \mu \mathrm{l}$ of suspension.

\subsubsection{Bronchoalveolar lavage and tissue collection}

Mice ( $\mathrm{n}=6-9$ per group, dose, and time point) were humanely sacrificed at 1, 7, 28, and $84 \mathrm{~d}$ postexposure. Mice received an intraperitoneal injection of $>100 \mathrm{mg}$ of sodium pentobarbital (Fatal-Plus Solution, Vortech Pharmaceutical, Inc., Dearborn, MI, USA) per kg of body weight, and were subsequently exsanguinated. The first set of mice was used to collect Bronchoalveolar lavage fluid (BALF) as previously described [87]. In brief, following exsanguination, the trachea was exposed and cannulated. USP-grade PBS was flushed into the lungs then recovered and divided into cellular and acellular using centrifugation. The cellular fraction was used to determine the total cell count using a hemocytometer in addition to cell differentials. The acellular fraction was used for cytotoxicity measurements and protein analysis. The second set of mice ( $n=4-6$ per group, dose, and time point) was used for tissue collection to determine gene expression changes. Following an intraperitoneal injection of sodium pentobarbital, the left lung lobe was removed and frozen at $-80{ }^{\circ} \mathrm{C}$ for gene expression analysis as previously described [164].

\subsubsection{BALF macrophages and imaging}

The cellular fraction collected from BALF was collected and imaged using electron microscopy using methods previously described [180]. In brief, the cellular fraction from BALF was collected and stored in Karnovsky's fixative solution (2\% paraformaldehyde/2.5\% glutaraldehyde in sodium cacodylate buffer) and agarose. Cells were postfixed using $1 \%$ Osmium tetroxide and stained with $1 \%$ Tannic acid and $0.5 \%$ uranyl acetate followed by dehydration in $100 \%$ ethanol and lastly infiltrated with propylene 
oxide and LX-112 epon. Sections were cut and embedded on copped grids for imaging with JEOL JEM 1400 transmission electron microscope (JEOL USA) with an AMT XR-81 M-B digital camera.

\subsubsection{In vivo toxicity assessments}

Cytotoxicity: Lactate dehydrogenase $(\mathrm{LDH})$ is a cytosolic enzyme that can be measured as an indicator of cellular damage. LDH activity in the acellular fluid was quantified using a Cobas Mira chemistry analyzer (Roche Diagnostic Systems; Montclair, IN). This quantification is obtained through the detection of the oxidation of lactate coupled to the reduction of nicotinamide adenine dinucleotide at a spectrophotometric setting of $340 \mathrm{~nm}$.

Cell Counts and Differentials: Total cell counts in BALF were quantified using a hemocytometer. Cells from BALF were mounted on slides using a Cytospin 4 (Thermo Fischer) and were fixed and stained using a Hema 3 kit (Fisher Scientific; Kalamazoo, MI) to differentiate cell types. 300 cells were counted, and the percentage of macrophages, neutrophils, and eosinophils was determined. The percentage was then multiplied by the total number of cells to quantify the total number of each cell type. Cells were also imaged using standard light microscopy at 40x magnification.

Protein Analysis: Rodent MAP 4.0 Mouse Sample Testing from Ampersand Biosciences (Saranac Lake, NY) was used to quantify 42 proteins present in BALF at 7,28 , and $84 \mathrm{~d}$ post exposure. The data is presented in a heat map representing the fold change from control (DM).

Gene Expression: mRNA was isolated from the left lung and converted to cDNA using previously described methods [164]. To assess inflammation, changes in relative fold change for six genes were calculated using the $2-\Delta \Delta C$ t method. The six genes measured include $I l-1 b, I l-6, C c l 2, C c l 22, C x c l 2$, and Spp1. 


\subsubsection{In vitro toxicity study design and toxicity assessments}

Submerged cultures of differentiated macrophage from human peripheral blood monocyte cell line (THP-1) were used to screen the nine CNT/Fs for their toxicity potential In vitro. A mechanism-based screening approach was used, and the nine CNT/Fs were screened for toxicity (LDH, WST-1), Nuclear factor- $\kappa \mathrm{B}(\mathrm{NF}-\kappa \mathrm{B})$ activation (inflammation), Inflammasome activation (IL-1 $\beta$ and IL-18) and Pyroptosis (Caspase-1). Finally, functional innate immune changes due to exposure of the nine CNT/Fs was evaluated by challenging the CNT/F exposed cells with green fluorescent protein Escherichia coli (GFP E. coli) and quantifying the relative uptake by flow cytometer.

\subsubsection{Macrophage culture, differentiation and priming}

Human peripheral blood monocyte cell line, THP-1 cells, were cultured, differentiated, and primed using procedures described previously $[180,181]$. The human monocyte cells (THP-1) were obtained from the American Type Culture Collection (ATCC \# TIB 202) and were cultured in HEPES buffered RPMI-1640 media supplemented with $10 \%$ fetal bovine serum (FBS), $100 \mu \mathrm{g} / \mathrm{mL}$ penicillinstreptomycin, and $50 \mu \mathrm{M}$ of $\beta$ mercaptoethanol. The THP-1 monocytes were differentiated to macrophages by treating the cells with fresh culture media supplemented with $150 \mathrm{nM} 1 \alpha, 25$-DihydroxyVitamin D3 (Vit D3; MilliporeSigma, MA; \# 679101) for 48 h. Cells were subsequently treated for $12 \mathrm{~h}$ with media supplemented with $10 \mathrm{nM}$ Phorbol, 12-myristate, 13-acetate (PMA, MilliporeSigma, MA; \# P8139). For the inflammasome activation, the differentiated THP-1 cells were primed by co-exposing particulate matter with $10 \mathrm{ng} / \mathrm{ml}$ Lipopolysaccharide (LPS; MilliporeSigma, MA; \# L-4516) to induce the transcription of pro-IL-1 $\beta$. Cells were cultured in a humidified incubator maintained at $370 \mathrm{C}$ and $5 \%$ $\mathrm{CO} 2$. 


\subsubsection{CNT/F Aqueous Stock Preparation and Dispersion in Cell Culture Media}

$\mathrm{CNT} / \mathrm{F}$ aqueous stock was prepared by dispersing the $\mathrm{CNT} / \mathrm{F}$ dry powder at $2 \mathrm{mg} / \mathrm{ml}$ concentration in dispersion medium [DM; $0.6 \mathrm{mg} / \mathrm{ml}$ mouse serum albumin $+0.01 \mathrm{mg} / \mathrm{ml}$ 1,2-dipalmitoyl-sn-glycero-3phosphotidyl (DPPC) in phosphate-buffered saline (PBS) without calcium and magnesium] [166]. CNT/F dispersion in the aqueous stock was achieved by sonicating for $5 \mathrm{~min}$ at $70 \%$ amplitude using a cup horn sonicator (Sonics VibraCell with Cup-type Sonicator; Newton, CT) immersed in continuous flowing cold water. The samples were vortexed intermittently after every minute for $10 \mathrm{~s}$. The aqueous stock was dispersed in cell culture media by diluting to highest test concentration and performing probe tip sonication (Branson Sonifer 450, continuous output) for a total of $2 \mathrm{~min}$, with $10 \mathrm{~s}$ vertexing after every $30 \mathrm{~s}$. Other test concentrations were obtained by serial dilution.

\subsubsection{Cell viability and membrane damage}

Cell viability with CNT/F exposure was determined using WST-1 cell proliferation reagent (MilliporeSigma, MA; \#5015944001). Membrane integrity of the cells after CNT/F exposure was assessed using CytoTox-One homogenous membrane integrity assay (Promega, WI; \# G7892). Cells were plated on a 96 well plate at 60,000 cells per cm2 (19,200 cells per well; 1.92 x105 cells per ml) in $100 \mu 1$ of cell culture media. The differentiated cells were challenged for $24 \mathrm{~h}$ with fresh media containing the nine CNT/F's at eight serial doses of $0,1.87 \mu \mathrm{g} / \mathrm{ml}, 3.75 \mu \mathrm{g} / \mathrm{ml}, 7.5 \mu \mathrm{g} / \mathrm{ml}, 15 \mu \mathrm{g} / \mathrm{ml}, 30 \mu \mathrm{g} / \mathrm{ml}, 60 \mu \mathrm{g} / \mathrm{ml}$, and $120 \mu \mathrm{g} / \mathrm{ml}$. These concentrations in terms of surface area corresponds to $0,0.58 \mu \mathrm{g} / \mathrm{cm} 2,1.17 \mu \mathrm{g} / \mathrm{cm} 2$, $2.34 \mu \mathrm{g} / \mathrm{cm} 2,4.68 \mu \mathrm{g} / \mathrm{cm} 2,9.37 \mu \mathrm{g} / \mathrm{cm} 2,18.75 \mu \mathrm{g} / \mathrm{cm} 2$, and $37.5 \mu \mathrm{g} / \mathrm{cm} 2$ respectively. At $22 \mathrm{~h}$ post exposure, half of the wells for each dose tested (4 of 8 wells) were lysed with $1 \%$ Triton X-100 (MilliporeSigma, MA; \#T8787). At $24 \mathrm{~h}, 70 \mu \mathrm{l}$ of the supernatant was transferred to a 96 well plate and spun on a centrifuge at $1000 \mathrm{RPM}$ for 5 minutes to settle the particulate/debris. $50 \mu 1$ of debris free supernatant was transferred to a fresh plate with equal amount of CytoTox-One reagent and the 
fluorescence was read at $560 \mathrm{~nm}$ excitation / $590 \mathrm{~nm}$ emission after 30 minutes. For determining the cellular viability, cells were challenged with fresh media containing 10\% volume/volume WST-1 cell proliferation reagent. After $2 \mathrm{~h}$ of incubation, the WST-1 consumption was recorded by measuring the absorbance at $450 \mathrm{~nm}$ subtracted with absorbance at $660 \mathrm{~nm}$ to account for turbidity/background. Experiments were performed three times with each dose tested in quadruplicates each turn $(\mathrm{n}=3 \times 4$ for each dose).

\subsubsection{NFאB activation}

NFאB activation after CNT/F exposure was monitored by using NF-kB SEAP Reporter THP-1 cells (THP1-Blue ${ }^{\mathrm{TM}} \mathrm{NF}-\kappa \mathrm{B}$ Cells; Invivogen, CA). NF- $\mathrm{BB}$ cells are derived from human THP1 monocytes

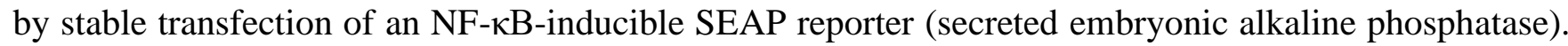
These cells allow monitoring of NF-kB activation by measuring SEAP expression. To maintain selection pressure, THP-1 growth media was treated with $10 \mu \mathrm{g} / \mathrm{ml}$ blasticidin (Invivogen, CA; \# ant-bl) every other passage. Cells were plated on a 96 well plate at 60,000 cells per cm2 (19,200 cells per well; 1.92 x105 cells per ml) in $100 \mu \mathrm{l}$ of cell culture media. After differentiation, cells were challenged with fresh media for 12 hours containing the nine CNT/F's at eight serial doses of $0,1.87 \mu \mathrm{g} / \mathrm{ml}, 3.75 \mu \mathrm{g} / \mathrm{ml}, 7.5 \mu \mathrm{g} / \mathrm{ml}, 15$ $\mu \mathrm{g} / \mathrm{ml}, 30 \mu \mathrm{g} / \mathrm{ml}, 60 \mu \mathrm{g} / \mathrm{ml}$, and $120 \mu \mathrm{g} / \mathrm{ml}$. These concentrations in terms of surface area corresponds to $0,0.58 \mu \mathrm{g} / \mathrm{cm} 2,1.17 \mu \mathrm{g} / \mathrm{cm} 2,2.34 \mu \mathrm{g} / \mathrm{cm} 2,4.68 \mu \mathrm{g} / \mathrm{cm} 2,9.37 \mu \mathrm{g} / \mathrm{cm} 2,18.75 \mu \mathrm{g} / \mathrm{cm} 2$, and $37.5 \mu \mathrm{g} / \mathrm{cm} 2$ respectively. After $12 \mathrm{~h}$ of exposure the supernatant was extracted and centrifuged at $1000 \mathrm{RPM}$ for 5 minutes. $20 \mu \mathrm{l}$ of the debris free cell supernatant was transferred to a 96 well plate containing $180 \mu \mathrm{l}$ of warm QUANTI-Blue ${ }^{\mathrm{TM}}$ solution (Invivogen, CA \#rep-qbs). The plate was incubated at $37{ }^{\circ} \mathrm{C}$ for $3 \mathrm{~h}$ and the change in color intensity to purple/blue due to secreted embryonic alkaline phosphatase (SEAP) was quantified by measuring absorbance at $635 \mathrm{~nm}$. 


\subsubsection{Caspase-1, IL-1 $\beta$ and IL-18}

Active caspase-1 was quantified using a fluorochrome inhibitor of caspase-1 (FAM-YVAD-FMK) (Immunochemistry Technologies, MN; Catalog \#98). The differentiated THP-1 cells were challenged with $30 \mu \mathrm{g} / \mathrm{ml}(9.37 \mu \mathrm{g} / \mathrm{cm} 2)$ of the nine CNT/F for $12 \mathrm{~h}$. The cells were washed with PBS and extracted using Accutase (Thermofisher, CA; \#4555-56). The detached cells were collected on ice and then centrifuged at $300 \mathrm{~g}$ for $5 \mathrm{~min}$. FAM-YVAD-FMK enters the cells freely and covalently binds to activated caspase-1. The FLICA reagent was diluted as per manufacturers recommendation and incubated with cells on ice for $15 \mathrm{~min}$ and washed with PBS. The FLICA labeled cells were fixed using the fixative provided by the manufacturer and the fluorescence was determined using a BD LSR II flow cytometer (BD Biosciences, CA). All experiments were performed using triplicate samples and at least 10,000 cells were acquired per treatment. The mean fluorescence was determined using FlowJo (FlowJo LLC, Oregon).

IL-1 $\beta$ and IL-18 production in differentiated and primed THP-1 culture supernatants exposed to $18.75 \mu \mathrm{g} / \mathrm{cm} 2(60 \mu \mathrm{g} / \mathrm{ml})$ of the nine CNT/Fs for $24 \mathrm{~h}$ was determined using Human IL-1 beta/IL-1F2 Quantikine ELISA (Cat\#DLB50, R\&D Systems, Minneapolis, MN) and Human IL-18 ELISA (Cat\#7620, MBL International Corporation, Woburn, MA) following the manufacturers recommendation.

\subsubsection{Phagocytosis functional assay}

Alteration in phagocytic capacity of macrophages exposed to the nine CNT/F was evaluated by challenging the CNT/F exposed macrophages with Escherichia coli GFP (ATCC \# 25922GFP). The differentiated macrophages were exposed to the nine CNT/F at $4.68 \mu \mathrm{g} / \mathrm{cm} 2(15 \mu \mathrm{g} / \mathrm{ml})$ for $24 \mathrm{~h}$. At $24 \mathrm{~h}$, the cells were washed and challenged with fresh media containing E. coli GFP at multiplicity of infection (MOI) of 1:25. In order for the bacteria to reach the cells at the bottom of the well, the plate containing the cells and bacteria was centrifuged at 1000 RPM for 10 min before placing them in an incubator at $37^{\circ} \mathrm{C}$. After $2 \mathrm{~h}$ of challenge, the cells were washed with PBS, harvested by trypsinization, and scraping, 
centrifuged at 1000 RPM for 5 min, and re-suspended in PBS. The cell-associated bacteria were quantified using a BD LSR II flow cytometer (BD Biosciences, CA). All experiments were performed using triplicate samples and at least 10,000 cells were acquired per treatment. The mean fluorescence was determined using FlowJo (FlowJo LLC, Oregon).

\subsubsection{Feature selection and principal component analysis}

To permit selection of the minimal number of features among all characterization and L-W properties that could be used to segregate each material investigated, feature selection was performed with a random forest-based approach [169] using the "Boruta" algorithm [170] in the R statistical environment [171]. The Boruta algorithm adds randomness to the variables in the dataset by creating shuffled copies of all variables ("shadow features"). "Boruta" iteratively assesses if each variable has a higher Z-score than the maximum Z-score of its shadow features. At each iteration, variables with Z-scores lower than shadow features are deemed unimportant and removed subsequently by the algorithm to capture all the important, interesting features one might have in the dataset with respect to a dependent variable, in this case each material itself. Then, using traditional, L-W, and combined variables retained after applying the "Boruta" algorithm, principal component analysis (PCA) was performed to identify significant patterns that explained the majority of the variations in the physicochemical properties among the different CNT/F materials investigated. PCA was performed using the prcomp command of the R statistical software (R Core Team, 2016).

\subsubsection{Statistics}

Data are presented as mean with standard error or standard deviation as indicated in the figure legends. Figures were prepared using SigmaPlot software (Systat Software, INC). Statistical analysis was performed using SAS/STAT software, Version 9.4 of the SAS system for Windows (SAS Institute, Cary, 
NC). Analysis used include one-way analysis of variance. Differences were considered significant at $\mathrm{p}<$

0.05. Post hoc comparisons were evaluated with Fishers LSD test. Some variables were transformed using the natural log prior to analysis to meet the model assumptions of homogeneous variance. 


\section{CHATER 3 FIGURES}
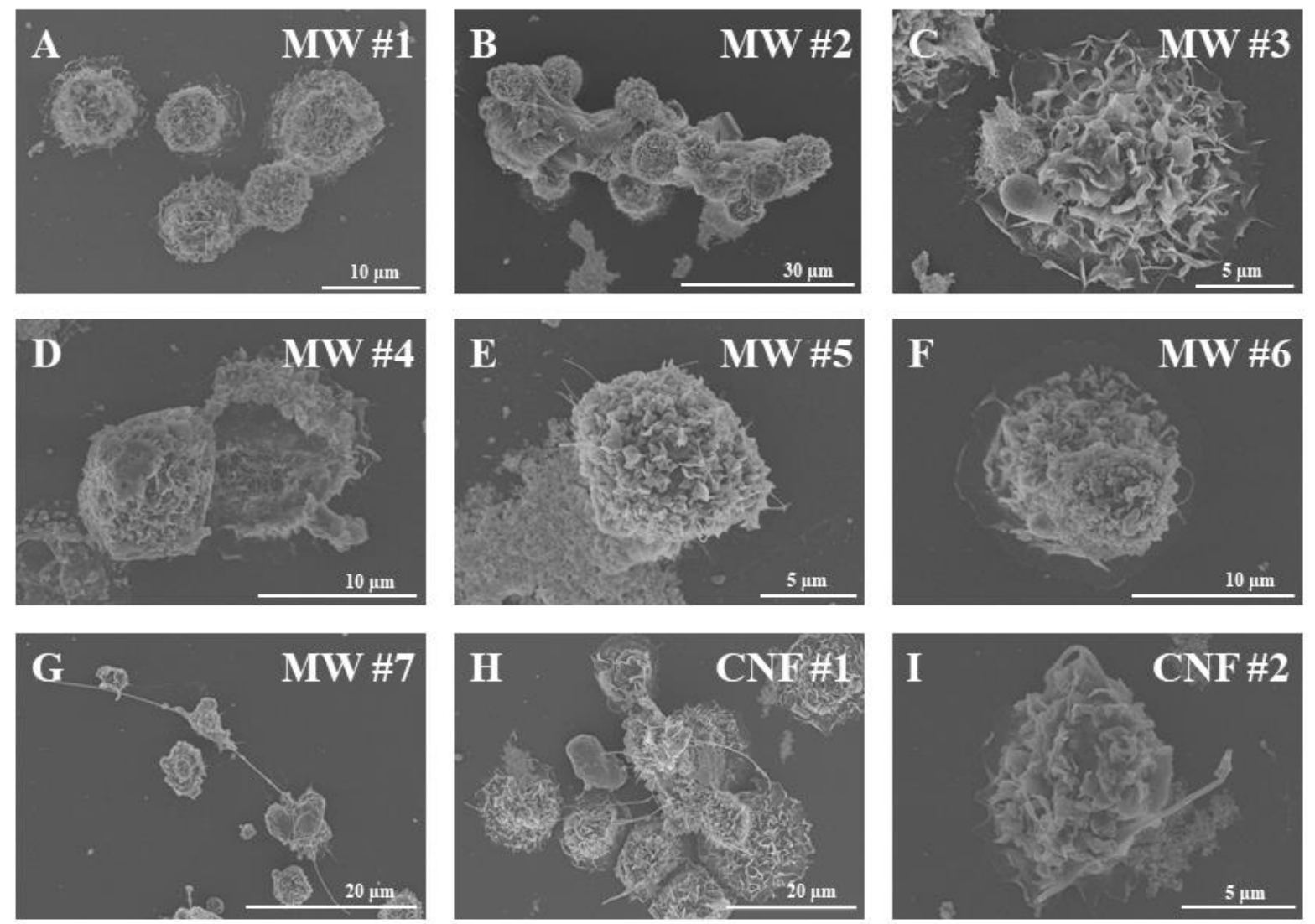

Figure 3.1. Representative electron microscopy images of macrophages isolated from bronchoalveolar lavage fluid at 7 days post-exposure. Smaller, spherically agglomerated particles are not highly visible due to their complete phagocytosis (MW \#1 and 3). Particles of large, bundled agglomerate size and singlets of larger nominal tube lengths and diameters are less readily phagocytosed due to the limitations of macrophage size and phagocytic capacity. These particles (MW \#2, 4-7, and CNF \#1-2) may induce frustrated phagocytosis and diminished lung clearance. 
A

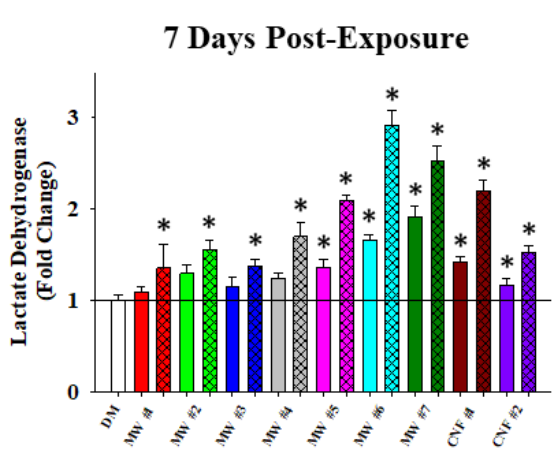

B

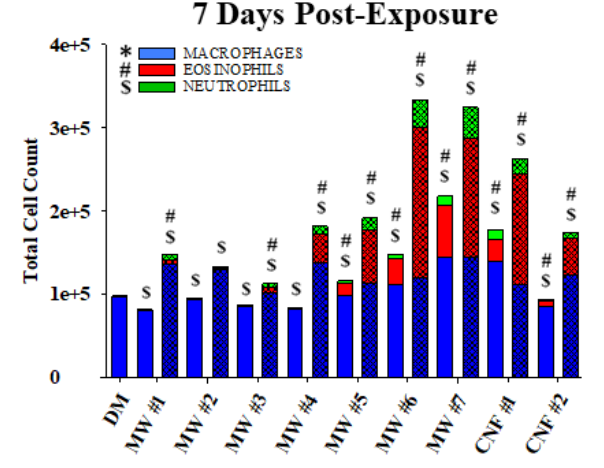

C $\quad-0.3 \quad \log$

1.4

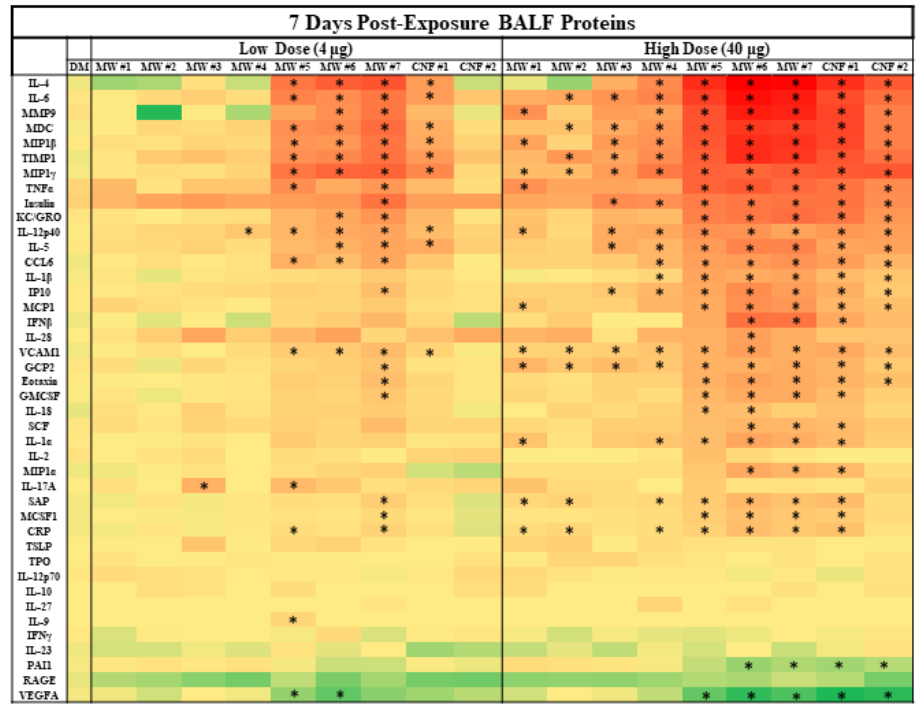

Figure 3.2. Inflammation outcomes at 7 days post exposure including lactate dehydrogenase (A), inflammatory cell infiltration (B), and bronchoalveolar lavage fluid proteins (C). While all materials induced significant inflammation in the high dose, and occasionally in the low dose, materials of larger nominal tube size (MW \#5-7, CNF \#1-2) were generally more likely to induce more severe or greater inflammatory responses compared to materials of smaller nominal tube size (MW \#1-4) $(* \mathrm{p}>0.05)$. 
A

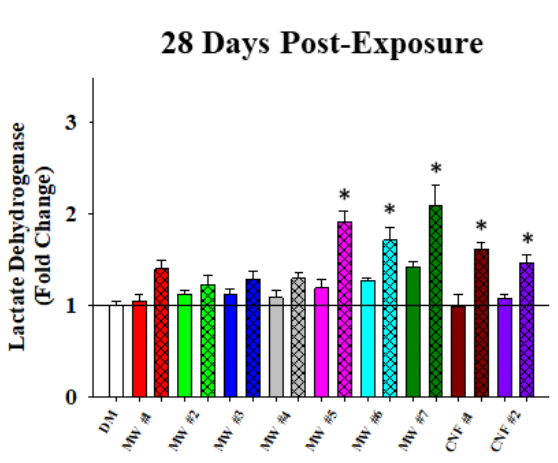

B

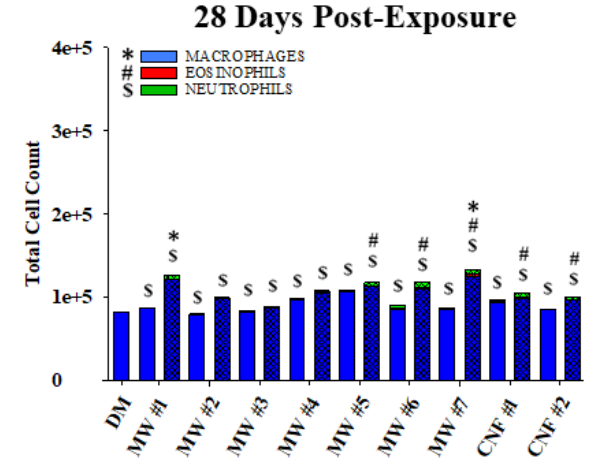

C $\quad-0.3 \quad \log 1.4$

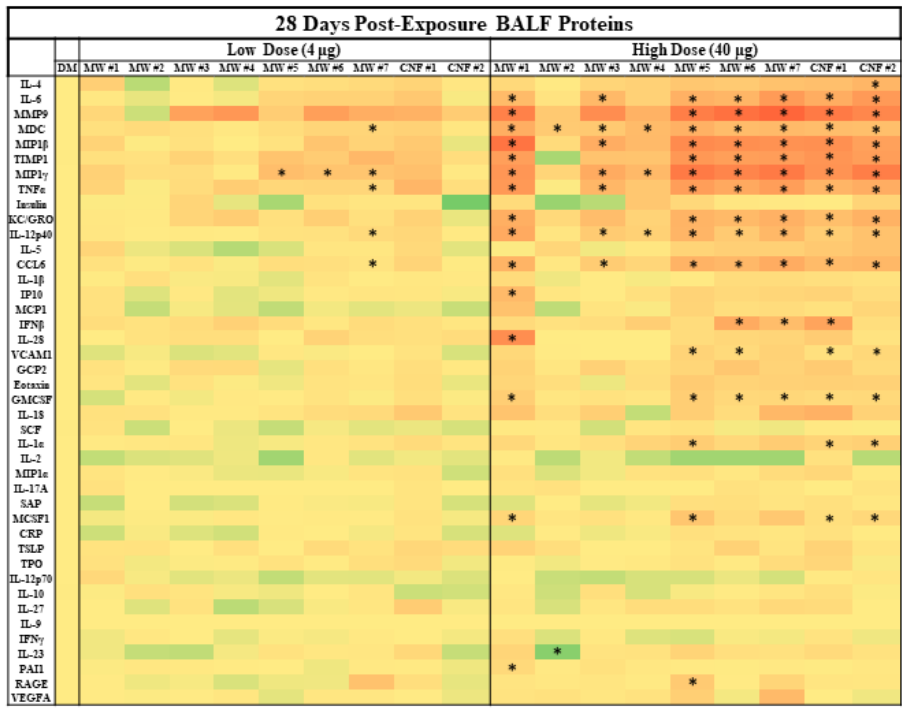

Figure 3.3. Inflammation outcomes at 28 days post exposure including lactate dehydrogenase (A), inflammatory cell infiltration (B), and bronchoalveolar lavage fluid proteins (C). Materials of larger nominal tube size (MW \#5-7, CNF \#1-2) generally induced significant inflammatory responses compared to materials of smaller nominal tube size (MW \#1-4) that were sustained through 28 days post-exposure $(* \mathrm{p}>0.05)$. 
A

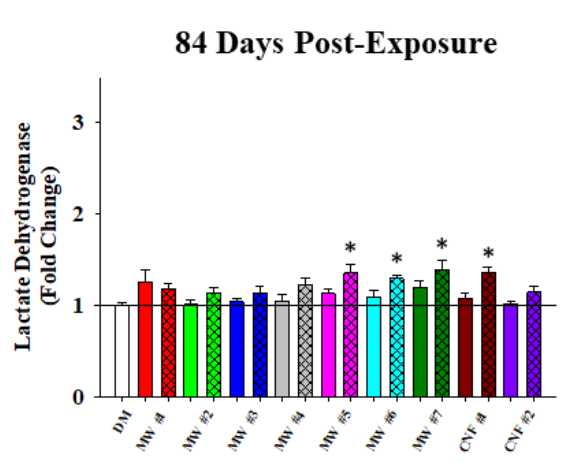

B

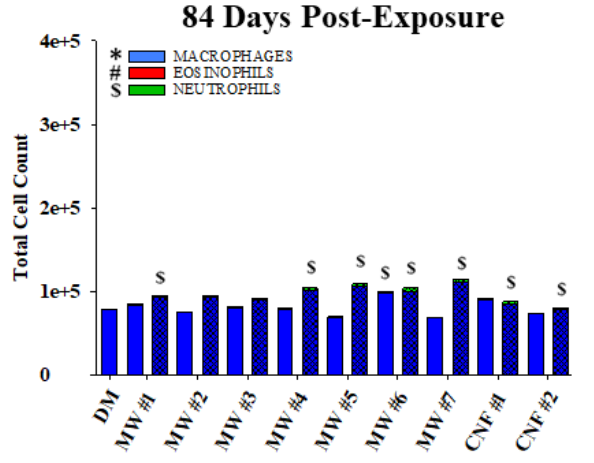

C $\quad-0.3 \quad \log 1.4$

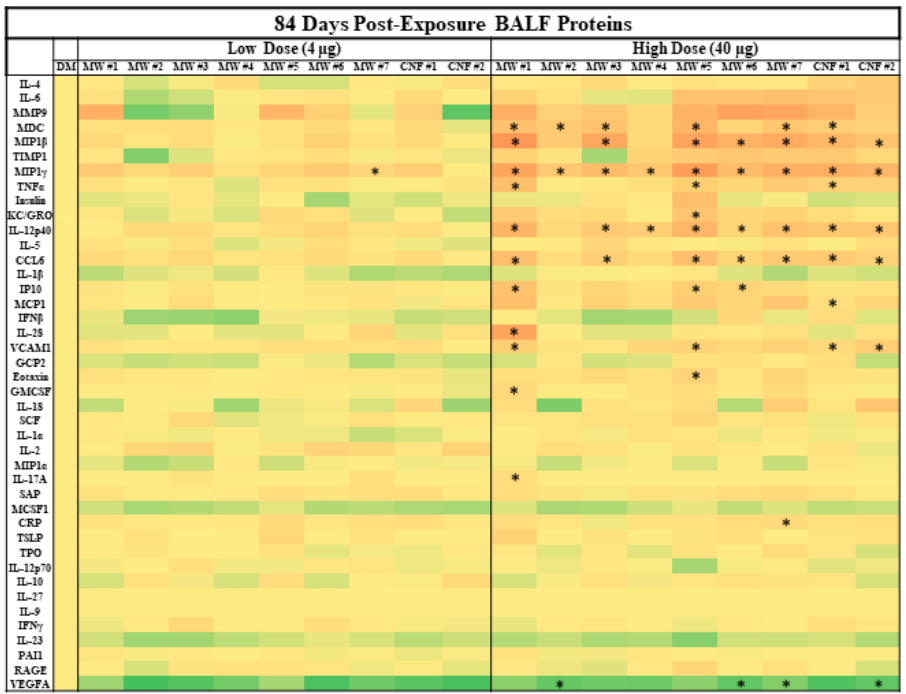

Figure 3.4. Inflammation outcomes at 84 days post exposure including lactate dehydrogenase (A), inflammatory cell infiltration (B), and bronchoalveolar lavage fluid proteins (C). While inflammation was trending toward resolution to baseline, MW \#5-7 and CN \#1 induced significant, persistent inflammation at this chronic time point. All other Materials achieved resolution $(* \mathrm{p}>0.05)$. 

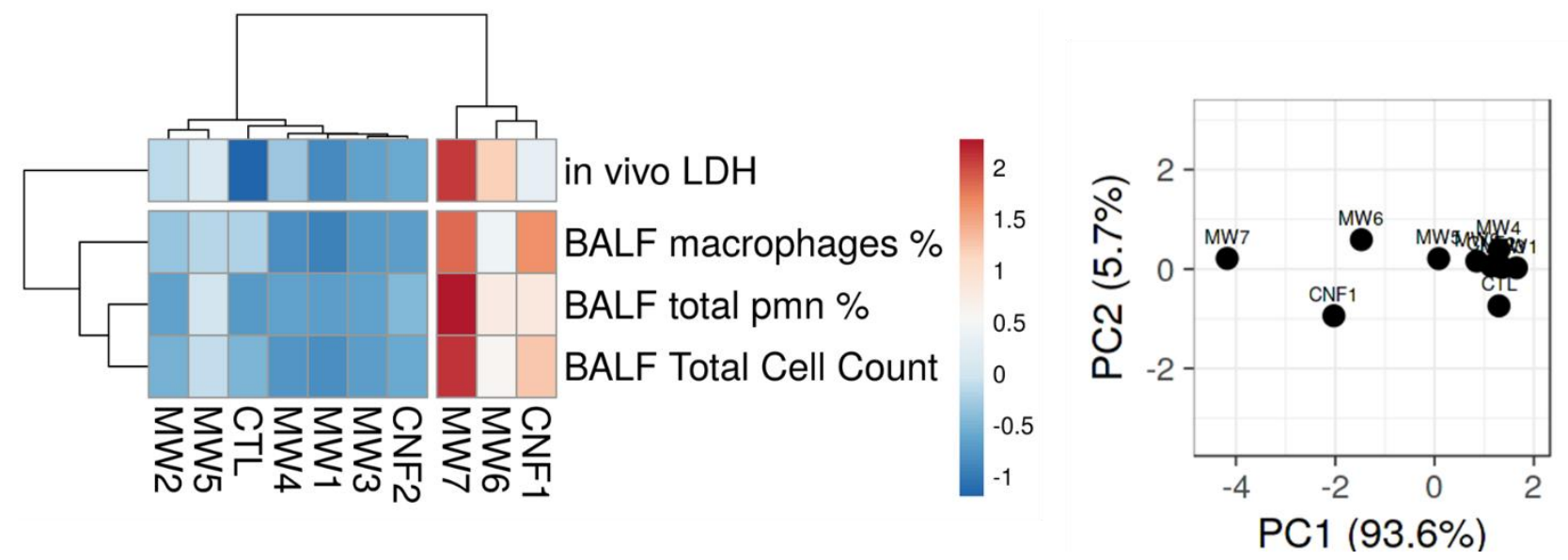

Figure 3.5. Hierarchical cluster analysis (HCA) and principal component analysis (PCA) grouping of inflammation outcomes alone including lactate dehydrogenase and inflammatory cell infiltration for the 4 $\mu \mathrm{g}$ dose at 7 days post-exposure. 

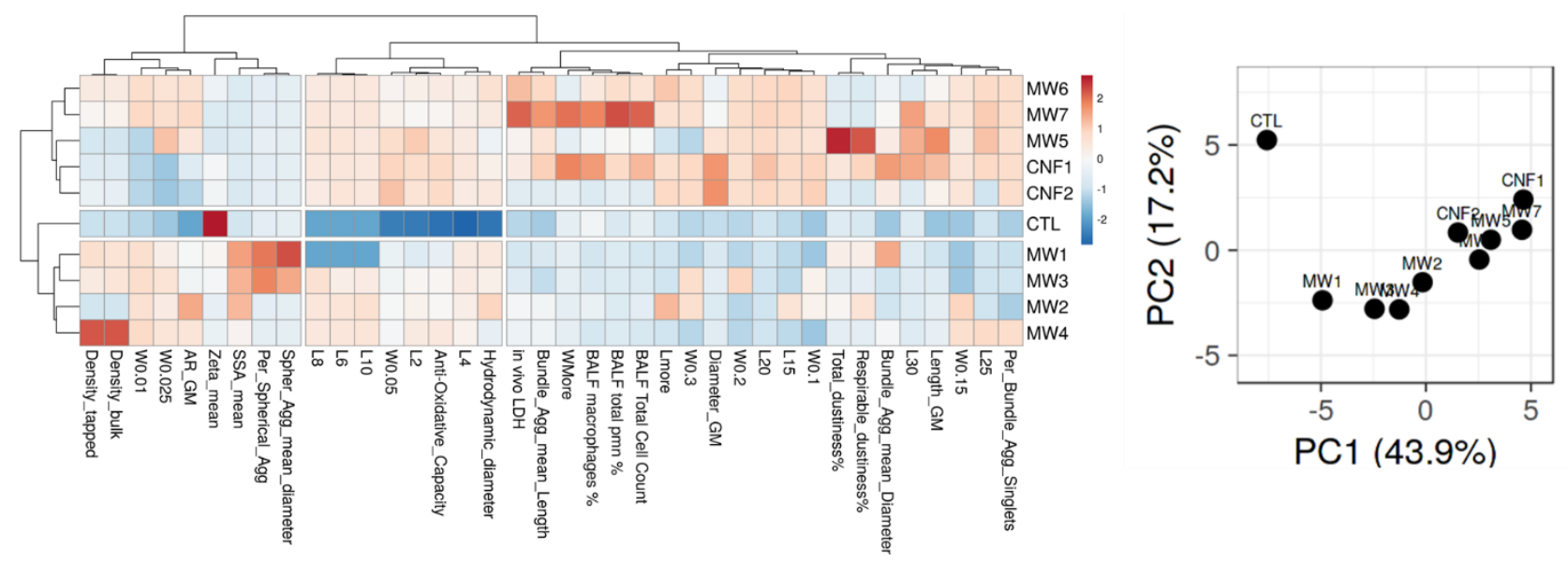

Figure 3.6. Hierarchical cluster analysis (HCA) and principal component analysis (PCA) grouping of inflammation outcomes for the $4 \mu \mathrm{g}$ dose at 7 days post-exposure and physicochemical characterization using 'all characterization' parameters 

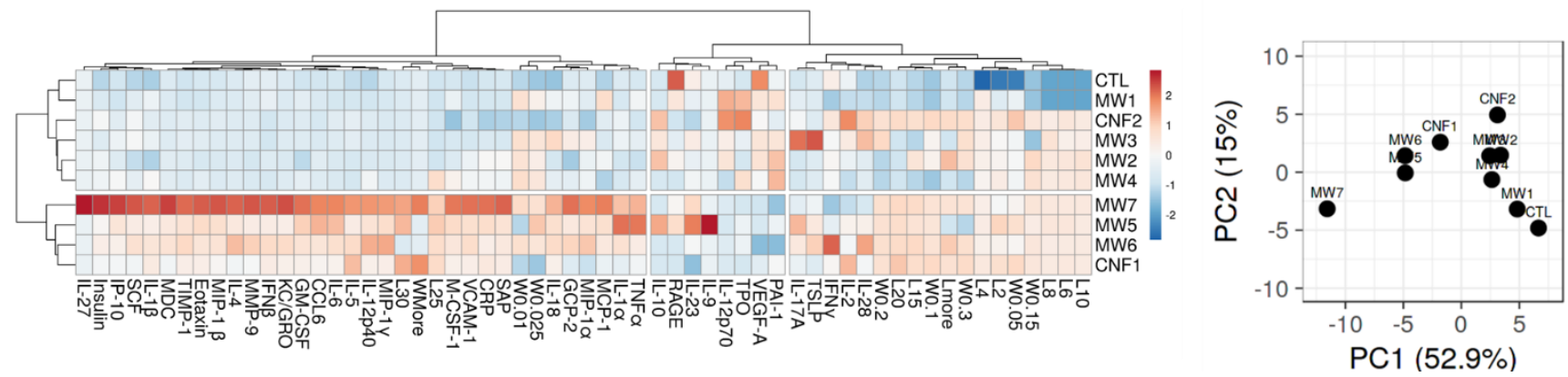

Figure 3.7. Hierarchical cluster analysis (HCA) and principal component analysis (PCA) grouping of inflammation outcomes for the $4 \mu \mathrm{g}$ dose at 7 days post-exposure and physicochemical characterization using length-width (L-W) parameters. 
A

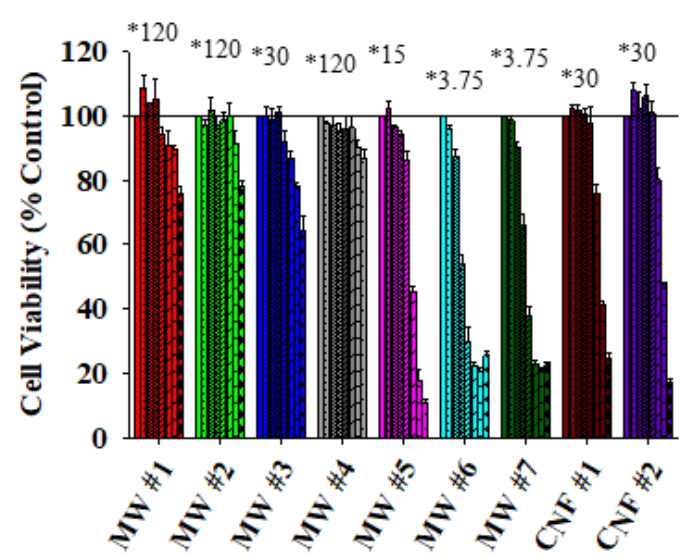

B

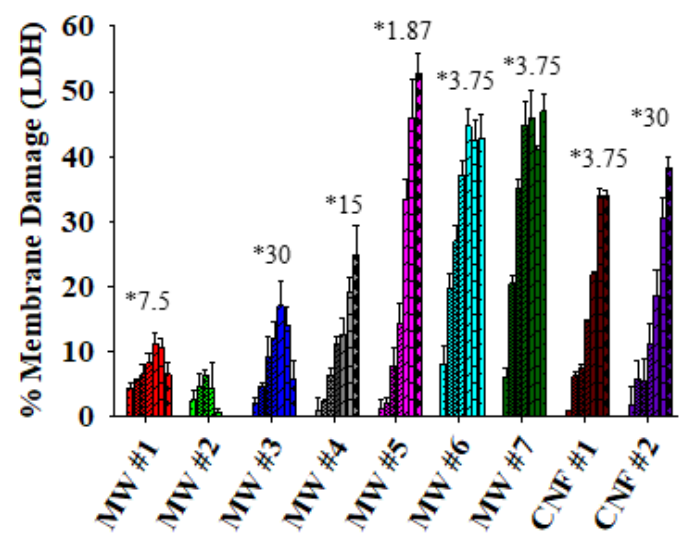

C

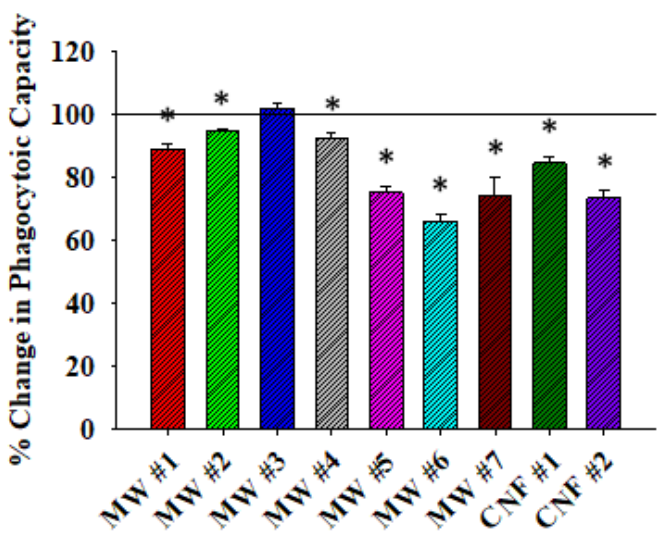

\footnotetext{
ए $0 \mathrm{mg} / \mathrm{ml} \quad 3.75 \mathrm{mg} / \mathrm{ml}$ шखया $15 \mathrm{mg} / \mathrm{ml} \rightleftharpoons 60 \mathrm{mg} / \mathrm{ml}$
}

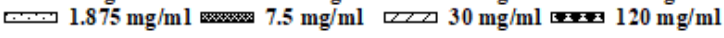

Figure 3.8. Toxicity assessment of inflammation in THP-1 macrophage cells exposed to CNT/F. (A) WST-1 cell proliferation assay was used to assess the viability of THP-1 cells following exposure to increasing concentrations $(01.875-120 \mu \mathrm{g} / \mathrm{ml})$ of $\mathrm{CNT} / \mathrm{F}$. The dose at which the particle significantly reduced cell viability is indicated with an asterisk $(\mathrm{p}<0.05)$. (B) Lactate dehydrogenase is indicative of membrane damage and was quantified in the cell supernatant following 24 hours of particle exposure. *p $<0.05$ fold change vs. control cells represented as a reference line. (C) Changes in phagocytic capacity of macrophages was assessed following $24 \mathrm{~h}$ treatment. Significant changes from control cells were indicated with an asterisk $(* \mathrm{p}<0.05)$. 
A

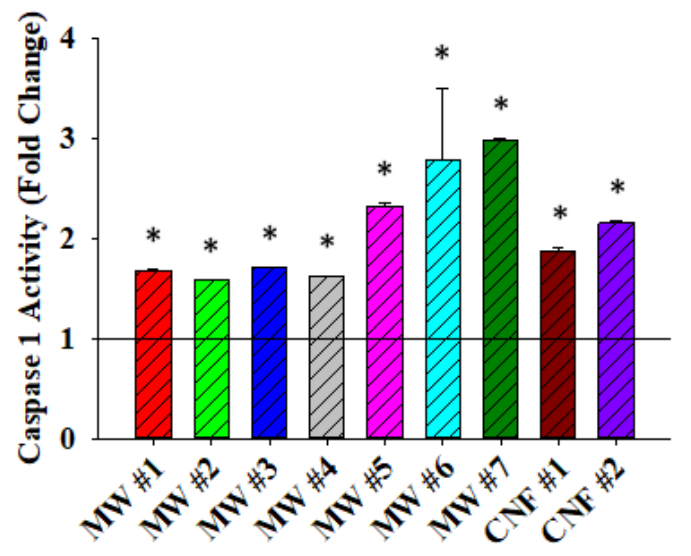

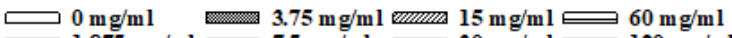

B

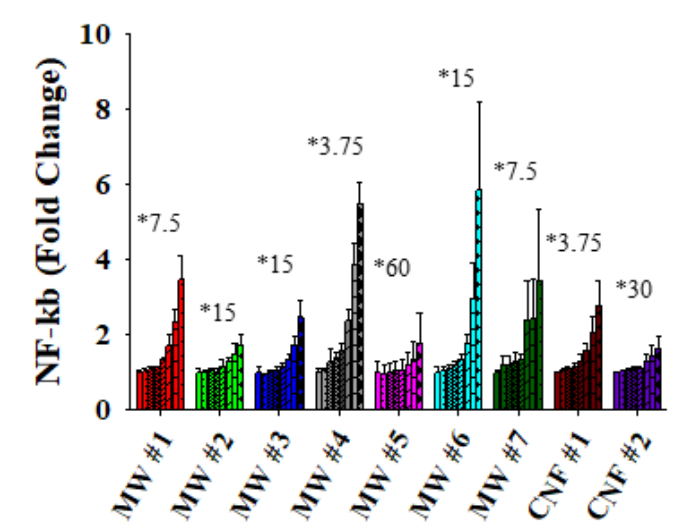

C

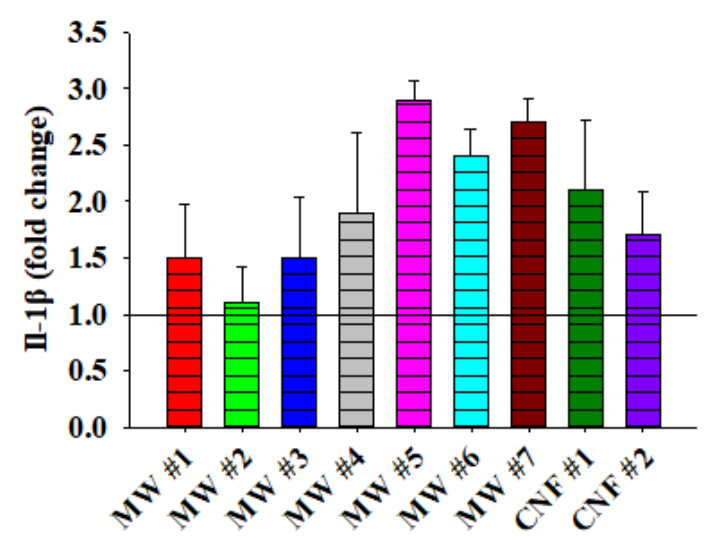

D

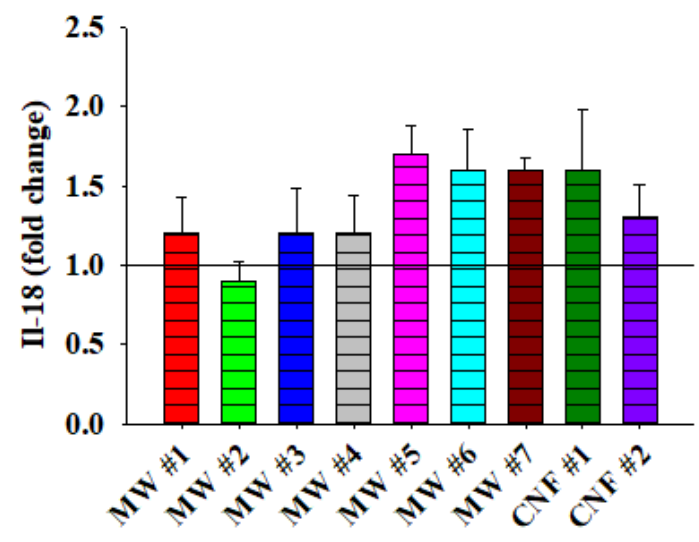

Figure 3.9. Inflammasome activation and toxicity assessment of THP-1 macrophage cells exposed to

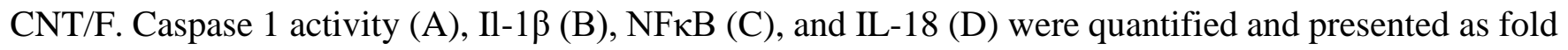
change from control. Significant changes from control cells were indicated with an asterisk $(* \mathrm{p}<0.05)$. 

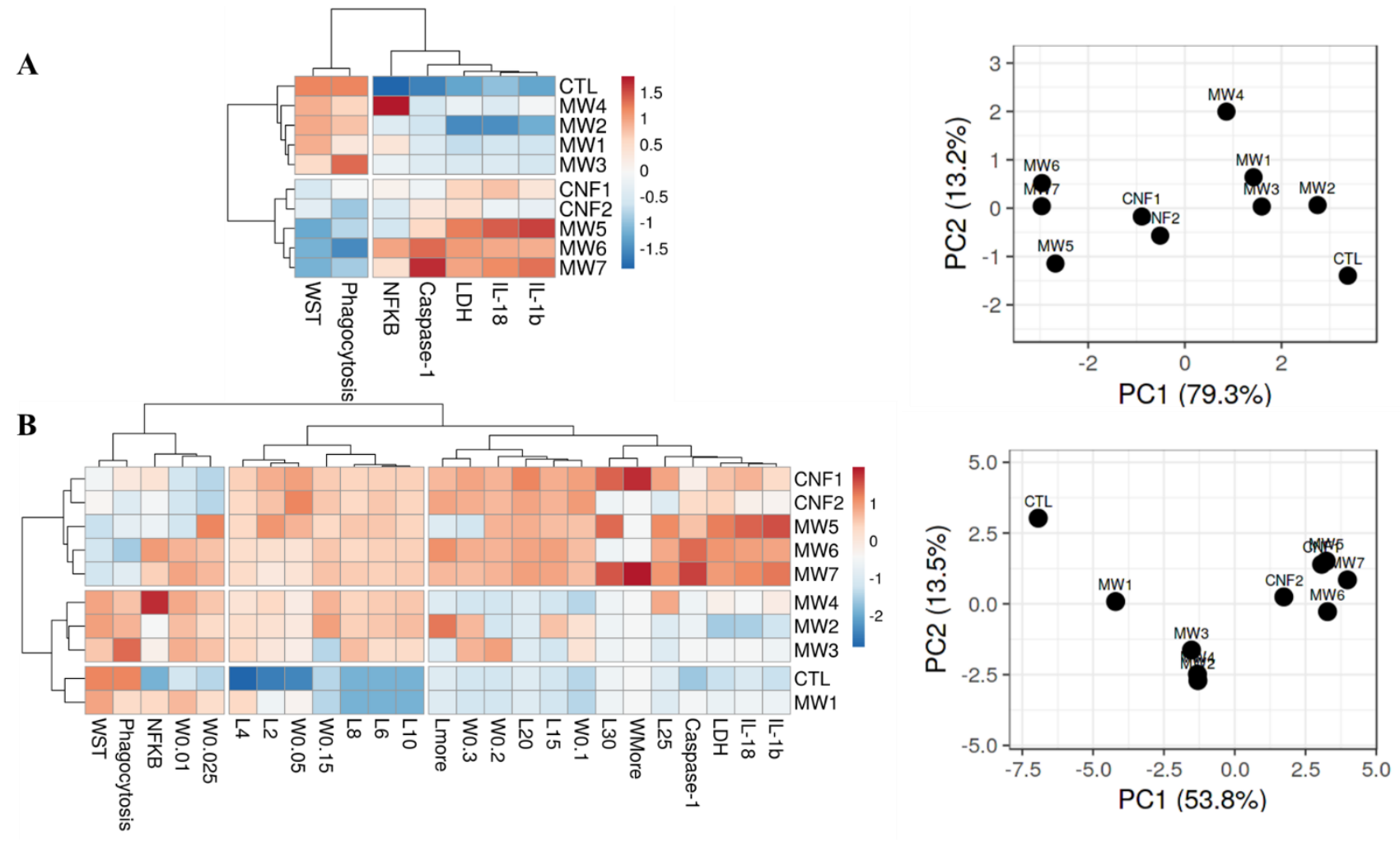

Figure 3.10. Hierarchical cluster analysis (HCA) and principal component analysis (PCA) grouping THP1 inflammation outcomes alone (A) and THP-1 inflammation outcomes with length- width (L-W) physicochemical characteristics (B). Similar groupings as in the in vivo findings were generated. 


\section{CHAPTER 3 SUPPLEMENTARY TABLES AND FIGURES}

Supplementary Table 3.1. Physical dimensions of CNT/F dispersed in isopropanol.

\begin{tabular}{|c|c|c|c|c|c|c|c|c|c|c|}
\hline & & MW \#1 & MW \#2 & MW \#3 & MW \#4 & MW \#5 & MW \#6 & MW \#7 & CNF \#1 & CNF \#2 \\
\hline \multirow{6}{*}{$\begin{array}{l}\text { Diameter } \\
(\mathrm{nm})\end{array}$} & $\begin{array}{r}\text { Company Reported } \\
\text { Diameter (nm) }\end{array}$ & $6-9$ & 10 & $10-15$ & $5-30$ & N/A & $70-80$ & 150 & 100 & 150 \\
\hline & $\begin{array}{r}\text { Geometric Mean } \\
(\mathrm{nm} \pm \mathrm{GSD})\end{array}$ & $13 \pm 1$ & $14 \pm 2$ & $20 \pm 2$ & $19 \pm 1$ & $63 \pm 1$ & $28 \pm 2$ & $37 \pm 2$ & $102 \pm 1$ & $103 \pm 1$ \\
\hline & $\begin{array}{r}\text { Arithmetic Mean } \\
(\mathrm{nm} \pm \mathrm{SE})\end{array}$ & $13 \pm 0$ & $16 \pm 1$ & $26 \pm 2$ & $20 \pm 1$ & $67 \pm 2$ & $38 \pm 3$ & $54 \pm 4$ & $110 \pm 3$ & $110 \pm 3$ \\
\hline & Median & 12 & 14 & 19 & 18 & 63 & 25 & 28 & 98 & 100 \\
\hline & Diameter Range & 6-29 & $6-216$ & $8-275$ & $8-133$ & $21-168$ & $8-218$ & $9-425$ & $40-397$ & $46-263$ \\
\hline & $\begin{array}{r}\text { Normal } \\
\text { Distribution } \\
\end{array}$ & Lognormal & & & & & & & & Lognormal \\
\hline \multirow{6}{*}{ Length $(\mu \mathrm{m})$} & $\begin{array}{r}\text { Company Reported } \\
\text { Length }(\mu \mathrm{m})\end{array}$ & 5 & N/A & $0.1-10$ & 100 & N/A & $\mathrm{N} / \mathrm{A}$ & N/A & $50-200$ & $50-200$ \\
\hline & $\begin{array}{r}\text { Geometric Mean } \\
(\mu \mathrm{m} \pm \mathrm{GSD})\end{array}$ & $0.67 \pm 1.81$ & $1.34 \pm 2.21$ & $1.10 \pm 2.00$ & $1.41 \pm 1.97$ & $4.39 \pm 2.07$ & $2.05 \pm 2.53$ & $2.88 \pm 4.26$ & $3.64 \pm 2.36$ & $2.16 \pm 2.31$ \\
\hline & $\begin{array}{r}\text { Arithmetic Mean } \\
(\mu \mathrm{m} \pm \mathrm{SE})\end{array}$ & $0.80 \pm 0.03$ & $1.79 \pm 0.10$ & $1.28 \pm 0.07$ & $1.84 \pm 0.13$ & $5.62 \pm 0.29$ & $3.42 \pm 0.37$ & $7.64 \pm 0.78$ & $5.23 \pm 0.36$ & $3.20 \pm 0.28$ \\
\hline & Median & 0.6607 & 1.5437 & 1.0148 & 1.2896 & 4.547 & 2.1503 & 2.3781 & 3.7273 & 2.0003 \\
\hline & Length Range & $0.1-3.6$ & $0.2-50.9$ & $0.1-8.5$ & $0.3-20.6$ & $1.2-25.8$ & $0.3-37.3$ & $0.1-49.1$ & $0.3-37.6$ & $0.4-42.7$ \\
\hline & $\begin{array}{r}\text { Normal } \\
\text { Distribution } \\
\end{array}$ & Lognormal & Lognormal & Lognormal & & & Lognormal & & Lognormal & Lognormal \\
\hline Aspect Ratio & $\begin{array}{r}\text { Aspect Ratio } \\
(\text { GeoMean } \pm \text { GSD }) \\
\end{array}$ & $53 \pm 2$ & $96 \pm 2$ & $50 \pm 2$ & $76 \pm 2$ & $69 \pm 2$ & $73 \pm 2$ & $78 \pm 3$ & $36 \pm 2$ & $21 \pm 2$ \\
\hline
\end{tabular}


Supplementary Table 3.2. Hydrodynamic diameter, zeta potential, and two-dimensional sizing of CNT/F agglomerates dispersed in physiologic dosing media.

\begin{tabular}{|c|c|c|c|c|c|c|c|c|c|c|c|}
\hline & & MW \#1 & $\begin{array}{c}\text { MW \#2 } \\
\text { Small } \\
\text { Agglomerates } \\
\end{array}$ & $\begin{array}{c}\text { MW \#2 } \\
\text { Large } \\
\text { Agglomerates } \\
\end{array}$ & MW \#3 & MW \#4 & MW \#5 & MW \#6 & MW \#7 & CNF \#1 & CNF \#2 \\
\hline \multirow{3}{*}{$\begin{array}{c}\text { Spherical } \\
\text { Agglomerates }\end{array}$} & $\%$ Spherical Agglomerated & 87 & 0 & 0 & 83 & 1 & 4 & 0 & 0 & 0 & 0 \\
\hline & $\begin{array}{r}\text { Spherical Agglomerate Mean } \\
\text { Diameter } \\
\mu \mathrm{m} \pm \mathrm{SE}\end{array}$ & $1.28 \pm 0.16$ & $\mathrm{~N} / \mathrm{A}$ & $\mathrm{N} / \mathrm{A}$ & $0.81 \pm 0.10$ & $\mathrm{~N} / \mathrm{A}$ & $\mathrm{N} / \mathrm{A}$ & $\mathrm{N} / \mathrm{A}$ & $\mathrm{N} / \mathrm{A}$ & $\mathrm{N} / \mathrm{A}$ & N/A \\
\hline & $\begin{array}{r}\text { Spherical Agglomerate Diameter } \\
\text { Geometric Mean } \\
\mu \mathrm{m}(\text { GSD) } \\
\end{array}$ & $0.96(2.01)$ & $\mathrm{N} / \mathrm{A}$ & $\mathrm{N} / \mathrm{A}$ & $0.66(1.84)$ & $\mathrm{N} / \mathrm{A}$ & $\mathrm{N} / \mathrm{A}$ & N/A & $\mathrm{N} / \mathrm{A}$ & $\mathrm{N} / \mathrm{A}$ & $\mathrm{N} / \mathrm{A}$ \\
\hline \multirow{5}{*}{$\begin{array}{c}\text { Bundled } \\
\text { Agglomerates }\end{array}$} & $\%$ Bundle Agglomerates/Singlets & 13 & N/A & $\mathrm{N} / \mathrm{A}$ & 17 & 99 & 96 & 100 & 100 & 100 & 100 \\
\hline & $\begin{array}{r}\text { Bundle Agglomerate Mean Length } \\
\mu \mathrm{m} \pm \mathrm{SE}\end{array}$ & $1.90 \pm 0.37$ & $3.80 \pm 0.38$ & $49.55 \pm 3.58$ & $1.11 \pm 0.25$ & $3.77 \pm 0.35$ & $6.27 \pm 0.44$ & $9.47 \pm 1.26$ & $11.32 \pm 1.08$ & $9.30 \pm 1.07$ & $2.96 \pm 0.36$ \\
\hline & $\begin{array}{r}\text { Bundle Agglomerate Geometric } \\
\text { Mean Length } \\
\mu \mathrm{m}(\mathrm{GSD})\end{array}$ & $1.66(1.71)$ & $3.09(1.82)$ & $47.94(1.31)$ & $0.72(2.98)$ & $2.92(2.05)$ & $5.17(1.94)$ & $5.90(2.57)$ & $7.91(2.49)$ & $6.18(2.49)$ & $2.11(2.25)$ \\
\hline & $\begin{array}{r}\text { Bundle Agglomerate Mean } \\
\text { Diameter } \\
\mu \mathrm{m} \pm \mathrm{SE}\end{array}$ & $0.38 \pm 0.12$ & $0.03 \pm 0.00$ & $9.50 \pm 2.24$ & $0.03 \pm 0.00$ & $0.10 \pm 0.01$ & $0.13 \pm 0.01$ & $0.08 \pm 0.01$ & $0.10 \pm 0.01$ & $0.21 \pm 0.01$ & $0.12 \pm 0.01$ \\
\hline & $\begin{array}{r}\text { Bundle Agglomerate Diameter } \\
\text { Geometric Mean } \\
\mu \mathrm{m}(\mathrm{GSD}) \\
\end{array}$ & $0.18(4.20)$ & $0.03(1.60)$ & $6.99(2.24)$ & $0.03(1.56)$ & $0.08(1.89)$ & $0.11(1.07)$ & $0.07(2.09)$ & $0.09(1.64)$ & $0.19(1.42)$ & $0.11(1.63)$ \\
\hline \multicolumn{2}{|c|}{$\begin{array}{l}\text { Hydrodynamic } \\
\text { Diameter (nm) }\end{array}$} & $660 \pm 19$ & \multicolumn{2}{|c|}{$771 \pm 33$} & $608 \pm 30$ & $478 \pm 24$ & $504 \pm 15$ & $714 \pm 24$ & $652 \pm 26$ & $615 \pm 19$ & $664 \pm 18$ \\
\hline \multicolumn{2}{|c|}{ Zeta Potential (pH 7.3) } & $-10.4 \pm 0.4$ & \multicolumn{2}{|c|}{$-12.1 \pm 0.6$} & $-11.1 \pm 0.06$ & $-12.0 \pm 0.4$ & $-13.5 \pm 0.8$ & $-11.8 \pm 0.6$ & $-13.2 \pm 0.5$ & $-11.1 \pm 0.8$ & $-11.3 \pm 0.5$ \\
\hline
\end{tabular}


Supplementary Table 3.3. Results of additional particle characterization of CNT/F.

\begin{tabular}{|c|c|c|c|c|c|c|c|c|c|c|}
\hline & & MW \#1 & MW \#2 & MW \#3 & MW \#4 & MW \#5 & MW \#6 & MW \#7 & CNF \#1 & CNF \#2 \\
\hline $\begin{array}{l}\text { Surface Area } \\
\left(\mathrm{m}^{2} / \mathrm{g} \pm \mathrm{SD}\right)\end{array}$ & & $237.7 \pm 1.0$ & $211.9 \pm 1.8$ & $218.6 \pm 1.2$ & $99.4 \pm 1.1$ & $25.2 \pm 0.4$ & $25.4 \pm 0.4$ & $24.7 \pm 0.4$ & $29.4 \pm 0.2$ & $18.0 \pm 0.2$ \\
\hline \multirow{2}{*}{ Dustiness } & $\begin{array}{r}\text { Dustiness Total } \\
(\%) \\
\end{array}$ & 3.8 & 2.9 & 0.3 & 0.5 & 14.0 & 0.2 & 0.2 & 4.9 & ND \\
\hline & $\begin{array}{r}\text { Dustiness } \\
\text { Respirable (\%) } \\
\end{array}$ & 0.84 & 1.10 & 0.20 & 0.20 & 2.40 & 0.08 & 0.09 & 1.40 & ND \\
\hline \multirow{2}{*}{ Density } & $\begin{array}{r}\text { Bulk Density } \\
(\mathrm{g} / \mathrm{cm} 3) \\
\end{array}$ & 0.087 & 0.007 & 0.082 & 0.169 & 0.007 & 0.075 & 0.061 & 0.020 & 0.032 \\
\hline & $\begin{array}{r}\text { Tapped Density } \\
(\mathrm{g} / \mathrm{cm} 3)\end{array}$ & 0.119 & 0.008 & 0.095 & 0.222 & 0.010 & 0.095 & 0.073 & 0.028 & 0.045 \\
\hline Endotoxin & & BLD & BLD & BLD & BLD & BLD & BLD & BLD & BLD & BLD \\
\hline PAH & & BLD & BLD & BLD & BLD & BLD & BLD & BLD & BLD & BLD \\
\hline \multirow{2}{*}{ Metal Catalyst } & $\% \mathrm{Fe}$ & 0.317 & 1.725 & 1.603 & 3.423 & 0.270 & 5.006 & 6.169 & 1.168 & 1.142 \\
\hline & $\% \mathrm{Al}$ & 0.310 & 0.028 & 2.116 & 0.019 & $\mathrm{~N} / \mathrm{A}$ & 0.035 & N/A & 0.006 & 0.013 \\
\hline \multirow{2}{*}{ TGA } & $\begin{array}{r}\text { TGA -Avg onset } \\
\text { oxidation, }{ }^{\circ} \mathrm{C}\end{array}$ & $550 \pm 2$ & $603 \pm 2$ & $575 \pm 0$ & $560 \pm 2$ & $735 \pm 2$ & $581 \pm 0$ & $592 \pm 0$ & $593 \pm 0$ & $694 \pm 0$ \\
\hline & $\begin{array}{r}\text { TGA - Mean } \\
\text { Residual Ash, \% } \\
\end{array}$ & $1.74 \pm 0.01$ & $3.98 \pm 0.26$ & $8.21 \pm 0.26$ & $4.75 \pm 0.07$ & $1.11 \pm 0.28$ & $7.88 \pm 0.15$ & $8.95 \pm 0.29$ & $1.79 \pm 0.12$ & $2.21 \pm 0.16$ \\
\hline $\begin{array}{l}\text { Anti-oxidative } \\
\text { Capacity }\end{array}$ & $\%$ & $64.53 \pm 23.91$ & $75.41 \pm 25.66$ & $76.34 \pm 27.37$ & $88.17 \pm 28.40$ & $91.49 \pm 21.37$ & $84.78 \pm 25.74$ & $77.27 \pm 19.96$ & $100.12 \pm 22.69$ & $99.80 \pm 24.19$ \\
\hline
\end{tabular}


Supplemental Table 3.4. Inflammatory cell counts of total cells and cell differentials listed as

means and standard errors of raw values

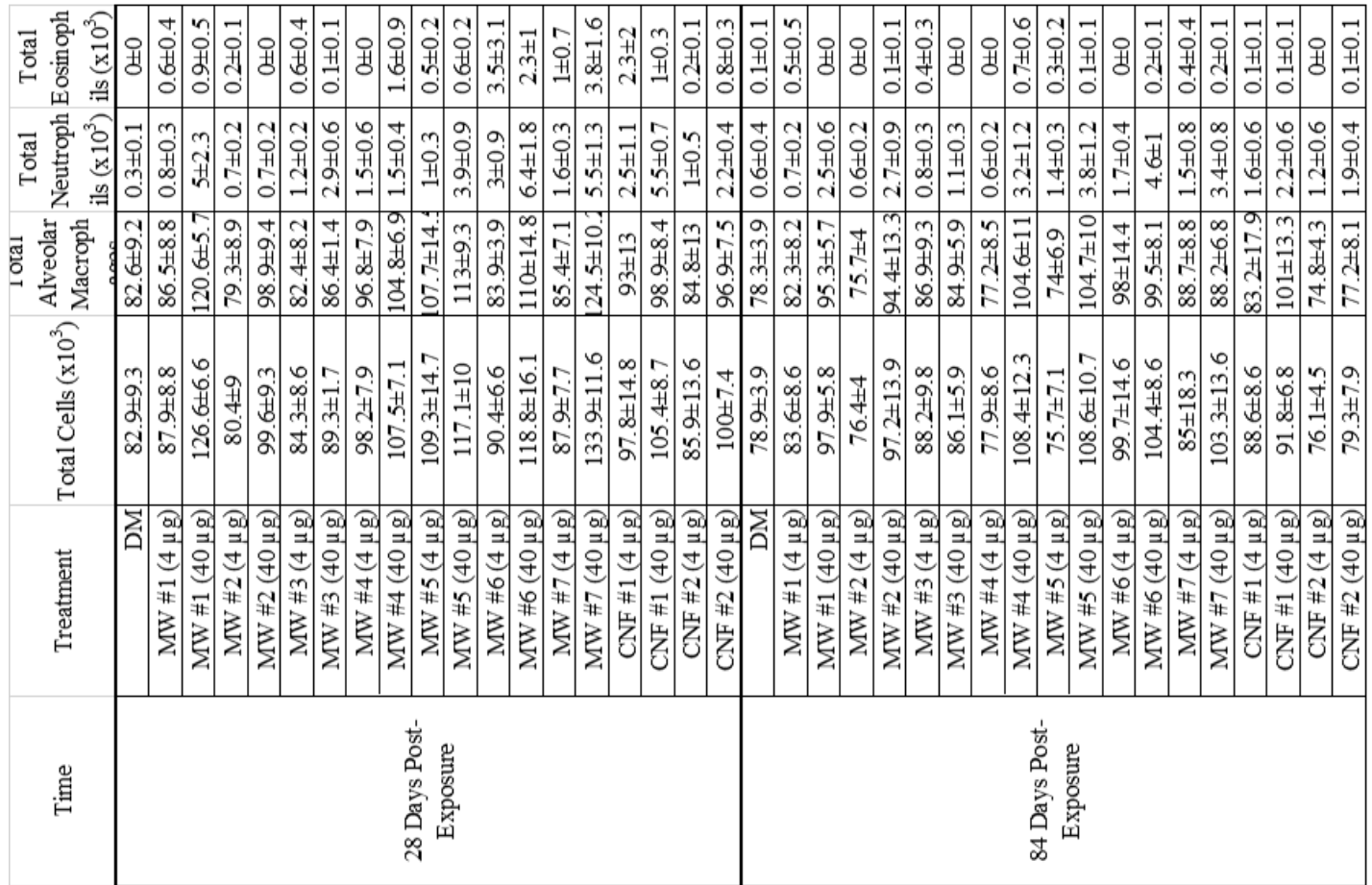

\begin{tabular}{|c|c|c|c|c|c|c|c|c|c|c|c|c|c|c|c|c|c|c|}
\hline 递 & $\ddot{\infty}$ & & & 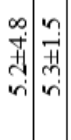 & 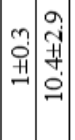 & 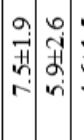 & 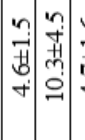 & 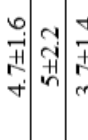 & 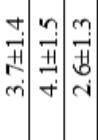 & & 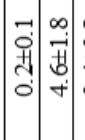 & 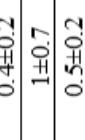 & 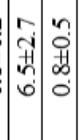 & 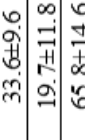 & 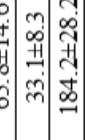 & 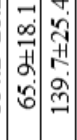 & 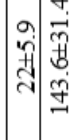 & 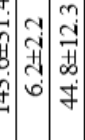 \\
\hline 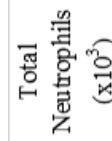 & 䓔荽 & & 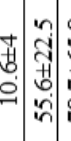 & 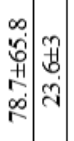 & 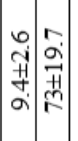 & 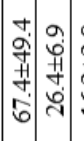 & 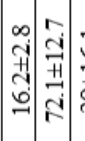 & 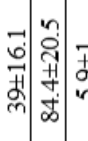 & 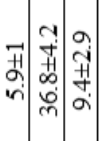 & & \begin{tabular}{cc|c}
1 & $\infty$ \\
0 & 0 \\
0 & 0 \\
0 & $\infty$ \\
0
\end{tabular} & $\begin{array}{l}0 \\
0 \\
0\end{array}$ & 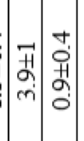 & & 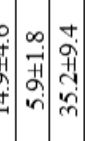 & 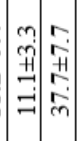 & 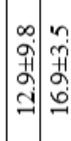 & $\hat{n}$ \\
\hline 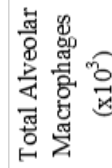 & 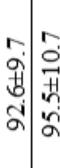 & 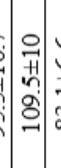 & 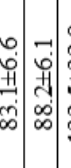 & 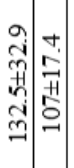 & 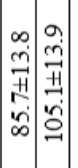 & 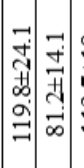 & 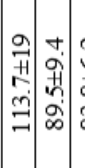 & 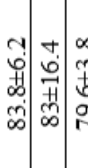 & 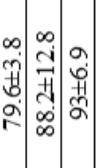 & 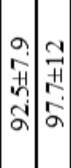 & 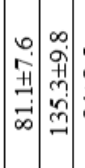 & 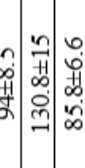 & 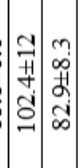 & 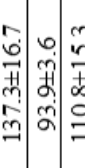 & 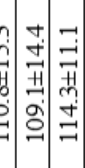 & 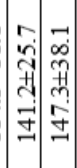 & 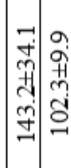 & 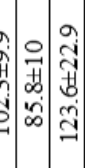 \\
\hline 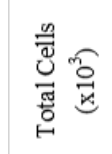 & 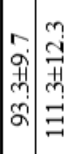 & & 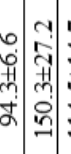 & 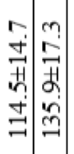 & 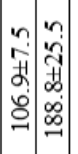 & 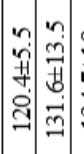 & 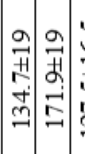 & 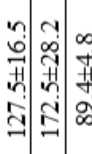 & 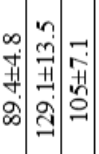 & 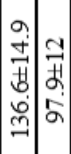 & 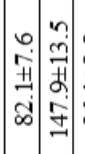 & 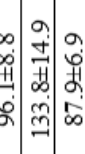 & 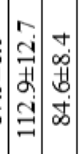 & 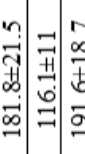 & 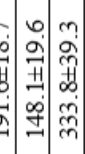 & 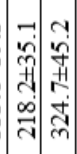 & 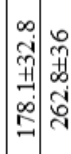 & 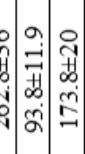 \\
\hline $\begin{array}{l}\text { 量 } \\
\text { 点 }\end{array}$ & 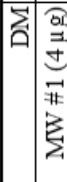 & 菲 & 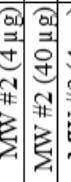 & 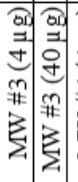 & 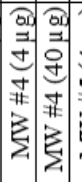 & 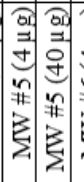 & 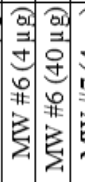 & 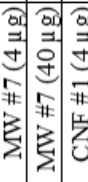 & 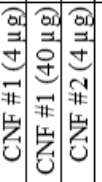 & 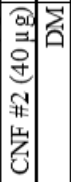 & 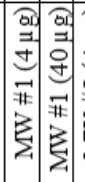 & 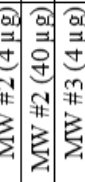 & $\begin{array}{lll}0 & 0 \\
0 \\
0 \\
0\end{array}$ & 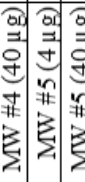 & $\begin{array}{ll}0 \\
0 \\
0\end{array}$ & 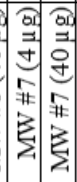 & 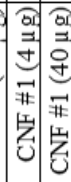 & $\begin{array}{lll}0 & 0 & 0 \\
0 \\
0\end{array}$ \\
\hline & & & & & & & & & & & & & & 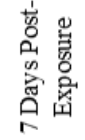 & & & & \\
\hline
\end{tabular}




\title{
Supplemental Table 3.5. Changes in mRNA expression of inflammatory cytokines and
}

\author{
chemoattractants listed as means and standard errors
}

\begin{tabular}{|c|c|c|c|c|c|c|c|c|c|c|c|}
\hline & \multicolumn{4}{|c|}{1 Day High Dose } & & & & & & & \\
\hline & & DM & MW \#1 & MW \#2 & MW \#3 & MW \#4 & MW \#5 & MW \#6 & MW \#7 & CNF\#1 & $\mathrm{CNF} \# 2$ \\
\hline Interleukin 6 & Il-6 & $1.00 \pm 0.24$ & $3.57 \pm 0.91$ & $6.32 \pm 1.24$ & $3.79 \pm 0.85$ & $10.53 \pm 2.40$ & $9.33 \pm 1.38$ & $9.52 \pm 2.34$ & $13.13 \pm 1.04$ & $7.87 \pm 1.81$ & $3.85 \pm 0.63$ \\
\hline Interleukin 1 beta & Il-1B & $1.00 \pm 0.23$ & $1.20 \pm 0.14$ & $1.27 \pm 0.26$ & $1.15 \pm 0.18$ & $1.96 \pm 0.45$ & $1.72 \pm 0.16$ & $1.75 \pm 0.48$ & $1.71 \pm 0.13$ & $1.97 \pm 0.29$ & $1.70 \pm 0.56$ \\
\hline C-C motif chemokine ligand 2 & $\mathrm{Ccl} 2$ & $1.00 \pm 0.30$ & $4.02 \pm 0.48$ & $1.78 \pm 0.29$ & $2.35 \pm 0.51$ & $7.48 \pm 2.25$ & $6.74 \pm 1.36$ & $4.73 \pm 1.16$ & $7.96 \pm 1.04$ & $6.63 \pm 1.21$ & $3.87 \pm 0.91$ \\
\hline C-C motif chemokine ligand 22 & Ccl22 & $1.00 \pm 0.08$ & $75 \pm 0.12$ & $1.04 \pm 0$ & $1.32 \pm$ & $1.78 \pm$ & $1.86 \pm 0.20$ & 1.61 & $1.87=$ & $1.85 \pm 0.17$ & $1.61 \pm 0.19$ \\
\hline Chemokine (C-X-C m & Cxcl2 & $1.00 \pm 0.55$ & \pm 0.21 & $1.84 \pm$ & $1.23 \pm$ & $2.37 \pm$ & $1.97 \pm 0.39$ & $1.49 \pm 0.63$ & $1.93 \pm 0.19$ & $2.71 \pm 0.69$ & $1.64 \pm 0.47$ \\
\hline oprotein 1 & Spp1 & $1.00 \pm 0.22$ & $13 \pm$ & $1.50 \pm$ & $1.25 \pm$ & $2.17 \pm$ & $4.34 \pm 0.68$ & $2.55 \pm 0.57$ & $5.67 \pm 0.52$ & $2.66 \pm 0.47$ & $1.36 \pm 0.25$ \\
\hline Metallothionein 1 & Mt1 & $1.00 \pm 0.22$ & $0.90 \pm 0.08$ & $0.99 \pm 0.06$ & $0.82 \pm 0.11$ & $1.40 \pm 0.13$ & $1.96 \pm 0.40$ & $1.80 \pm 0.63$ & $2.31 \pm 0.26$ & $1.22 \pm 0.18$ & $0.96 \pm 0.25$ \\
\hline Metallothionein 2 & Mt2 & $1.00 \pm 0.43$ & $1.31 \pm 0.18$ & $1.68 \pm 0.20$ & $1.15 \pm 0.29$ & $3.57 \pm 0.61$ & $5.13 \pm 1.20$ & $4.28 \pm 1.88$ & $6.99 \pm 0.90$ & $2.96 \pm 0.55$ & $1.63 \pm 0.55$ \\
\hline Heme oxygenase 1 & Hmox 1 & $1.00 \pm 0.14$ & $1.11 \pm 0.05$ & $1.07 \pm 0.07$ & $1.06 \pm 0.13$ & $1.08 \pm 0.06$ & $0.99 \pm 0.05$ & $1.05 \pm 0.02$ & $1.04 \pm 0.03$ & $1.13 \pm 0.06$ & $1.15 \pm 0.12$ \\
\hline
\end{tabular}

\begin{tabular}{|c|c|c|c|c|c|c|c|c|c|c|c|}
\hline & \multicolumn{4}{|c|}{1 Day Low Dose } & & & & & & & \\
\hline & & DM & MW \#1 & MW \#2 & MW \#3 & MW \#4 & MW \#5 & MW \#6 & MW \#7 & CNF\#1 & $\mathrm{CNF} \# 2$ \\
\hline ter & Il-6 & $1.00 \pm 0.10$ & $2.09 \pm 0.29$ & 19 & $1.97 \pm 0.25$ & $3.47 \pm 0.93$ & $2.44 \pm 0.24$ & $8.69 \pm 1.46$ & $5.99 \pm 0.73$ & $2.67 \pm 0.37$ & $1.97 \pm 0.15$ \\
\hline Interl & Il-1B & 5 & & & & & & & & & \\
\hline$-\mathrm{C}$ & & & & & & & & & & 3.4 & .28 \\
\hline$C$ & 22 & & 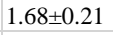 & & & & 13 & 22 & $2 .($ & 1.6 & 0.9 \\
\hline f) ligand & $\mathrm{xcl} 2$ & $1.00 \pm 0.20$ & $27 \pm 0.36$ & \pm 0.27 & 19 & $2.71 \pm 0.38$ & $3.15 \pm 0.35$ & $4.69 \pm 0.50$ & $3.92 \pm 0.37$ & $2.03 \pm 0.26$ & $1.79 \pm 0.24$ \\
\hline creted Phosopl & ppl & $00 \pm 0.12$ & $96 \pm 0.20$ & $85 \pm 0.07$ & $85 \pm 0.09$ & $80 \pm 0.19$ & $.86 \pm 0.08$ & $18 \pm 0.1$ & $97 \pm 0.12$ & $.92 \pm 0.0$ & \pm 0.15 \\
\hline
\end{tabular}
$\begin{array}{lllllllllllllll}\text { Cppl } & 1.00 \pm 0.12 & 0.96 \pm 0.20 & 0.85 \pm 0.07 & 0.85 \pm 0.09 & 0.80 \pm 0.19 & 0.86 \pm 0.08 & 1.18 \pm 0.11 & 0.97 \pm 0.12 & 0.92 \pm 0.09 & 0.99 \pm 0.15\end{array}$

Interleukin 1 beta

C-C motif chemokine ligand 2

$\mathrm{C}-\mathrm{C}$ motif chemokine ligand 22

Chemokine (C-X-C motif) ligand :

Secreted Phosophoprotein 1

Metallothionein 1

Metallothionein 2

Heme oxygenase 1
Interleukin 6

7 Day High Dose

\begin{tabular}{l|l|l|l|l|l|l|l|l|l} 
DM & MW \#1 & MW \#2 & MW \#3 & MW \#4 & MW \#5 & MW \#6 & MW \#7 & CNF \#1 & CNF \#2
\end{tabular}

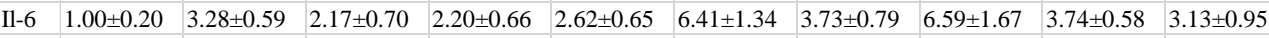
\begin{tabular}{c|c|c|c|c|c|c|c|c|c|c|} 
Il-1B & $1.00 \pm 0.11$ & $1.32 \pm 0.24$ & $1.66 \pm 0.30$ & $1.31 \pm 0.26$ & $1.71 \pm 0.16$ & $2.82 \pm 0.24$ & $3.14 \pm 0.75$ & $5.58 \pm 1.26$ & $2.47 \pm 0.15$ & $2.59 \pm 0.52$
\end{tabular} \begin{tabular}{lllllllll|l|l|l|l|l}
$\mathrm{Ccl} 2$ & $1.00 \pm 0.16$ & $8.95 \pm 2.20$ & $2.21 \pm 0.38$ & $3.92 \pm 1.17$ & $7.64 \pm 2.82$ & $15.57 \pm 4.20$ & $4.35 \pm 1.04$ & $14.81 \pm 3.95$ & $5.53 \pm 0.86$ & $5.46 \pm 1.80$
\end{tabular} \begin{tabular}{l|l|l|l|l|l|l|l|l|l|l}
$\mathrm{Ccl} 22$ & $1.00 \pm 0.11$ & $1.21 \pm 0.11$ & $1.14 \pm 0.13$ & $1.54 \pm 0.16$ & $1.97 \pm 0.26$ & $3.06 \pm 0.37$ & $2.69 \pm 0.33$ & $4.72 \pm 0.48$ & $3.03 \pm 0.35$ & $3.24 \pm 0.55$
\end{tabular} $\begin{array}{llllllllllllll}\text { Cxcl2 } & 1.00 \pm 0.13 & 5.21 \pm 0.96 & 3.50 \pm 0.55 & 2.66 \pm 0.44 & 3.31 \pm 0.78 & 9.84 \pm 1.34 & 8.66 \pm 2.13 & 14.41 \pm 3.06 & 8.13 \pm 0.60 & 5.58 \pm 1.15\end{array}$ $\begin{array}{lllllllllllllll}\text { Spp1 } & 1.00 \pm 0.14 & 6.98 \pm 1.30 & 2.55 \pm 0.31 & 5.39 \pm 0.88 & 7.00 \pm 3.28 & 16.70 \pm 2.84 & 3.38 \pm 0.99 & 6.80 \pm 1.57 & 5.08 \pm 1.35 & 2.65 \pm 0.73\end{array}$ $\begin{array}{llllllllllllll}\text { Mt1 } & 1.00 \pm 0.08 & 1.14 \pm 0.12 & 1.35 \pm 0.11 & 1.10 \pm 0.10 & 1.41 \pm 0.13 & 2.07 \pm 0.20 & 1.96 \pm 0.20 & 3.92 \pm 0.48 & 2.21 \pm 0.19 & 1.95 \pm 0.35\end{array}$ \begin{tabular}{l|l|l|l|l|l|l|l|l|l|l|l|l|} 
Mt2 & $1.00 \pm 0.13$ & $0.94 \pm 0.11$ & $1.24 \pm 0.12$ & $1.09 \pm 0.11$ & $1.74 \pm 0.26$ & $2.68 \pm 0.48$ & $2.73 \pm 0.52$ & $6.92 \pm 1.53$ & $2.85 \pm 0.31$ & $2.36 \pm 0.52$ \\
\hline
\end{tabular} $\begin{array}{llllllllllll}\text { Hmox } 1 & 1.00 \pm 0.06 & 1.14 \pm 0.11 & 0.93 \pm 0.04 & 1.02 \pm 0.03 & 1.15 \pm 0.07 & 1.47 \pm 0.08 & 1.21 \pm 0.07 & 1.97 \pm 0.18 & 1.37 \pm 0.09 & 1.39 \pm 0.19\end{array}$

Interleukin 6

Interleukin 1 beta

C-C motif chemokine ligand 2

C-C motif chemokine ligand 22

Chemokine (C-X-C motif) ligand :

Secreted Phosophoprotein 1

Metallothionein 1

Metallothionein 2

Heme oxygenase 1

$$
28 \text { Day High Dose }
$$
$\begin{array}{lllllllllllll}\text { Il-6 } & 1.00 \pm 0.17 & 4.89 \pm 1.36 & 1.27 \pm 0.31 & 3.61 \pm 0.87 & 2.79 \pm 0.46 & 3.61 \pm 1.31 & 2.92 \pm 0.27 & 3.24 \pm 0.39 & 2.74 \pm 0.31 & 2.85 \pm 0.32\end{array}$ \begin{tabular}{l|l|l|l|l|l|l|l|l|l|l} 
Il-1B & $1.00 \pm 0.08$ & $1.76 \pm 0.23$ & $1.08 \pm 0.25$ & $1.03 \pm 0.04$ & $1.32 \pm 0.05$ & $1.45 \pm 0.27$ & $1.66 \pm 0.17$ & $1.38 \pm 0.10$ & $1.33 \pm 0.18$ & $1.38 \pm 0.37$
\end{tabular} $\begin{array}{llllllllllllll}\mathrm{Cc} 2 & 1.00 \pm 0.12 & 6.72 \pm 2.17 & 1.06 \pm 0.12 & 3.08 \pm 0.86 & 2.06 \pm 0.50 & 2.10 \pm 0.75 & 2.08 \pm 0.28 & 2.18 \pm 0.12 & 2.30 \pm 0.20 & 1.84 \pm 0.22\end{array}$ \begin{tabular}{l|l|l|l|l|l|l|l|l|l|l}
$\mathrm{Ccl} 22$ & $1.00 \pm 0.05$ & $1.88 \pm 0.26$ & $1.21 \pm 0.13$ & $1.56 \pm 0.24$ & $1.96 \pm 0.24$ & $1.85 \pm 0.32$ & $2.14 \pm 0.18$ & $2.09 \pm 0.19$ & $2.26 \pm 0.17$ & $2.15 \pm 0.14$
\end{tabular} \begin{tabular}{l|l|l|l|l|l|l|l|l|l|l}
$\mathrm{Cxcl} 2$ & $1.00 \pm 0.16$ & $7.92 \pm 2.51$ & $1.28 \pm 0.24$ & $3.14 \pm 0.84$ & $3.15 \pm 0.65$ & $3.83 \pm 0.73$ & $6.06 \pm 1.25$ & $4.56 \pm 0.58$ & $4.34 \pm 0.17$ & $4.31 \pm 0.41$
\end{tabular} \begin{tabular}{l|l|l|l|l|l|l|l|l|l|l} 
Spp1 & $1.00 \pm 0.14$ & $4.36 \pm 0.97$ & $2.55 \pm 0.53$ & $8.34 \pm 4.80$ & $7.58 \pm 2.24$ & $14.22 \pm 6.10$ & $8.72 \pm 1.50$ & 12.942 .59 & $9.81 \pm 1.48$ & $9.60 \pm 1.25$ \\
\hline
\end{tabular} \begin{tabular}{l|l|l|l|l|l|l|l|l|l|l|l} 
Mt1 & $1.00 \pm 0.08$ & $1.17 \pm 0.21$ & $1.33 \pm 0.15$ & $1.37 \pm 0.19$ & $1.39 \pm 0.12$ & $1.75 \pm 0.08$ & $1.91 \pm 0.12$ & $1.88 \pm 0.15$ & $1.46 \pm 0.16$ & $1.85 \pm 0.18$
\end{tabular} $\begin{array}{lllllllllllll}\text { Mt2 } & 1.00 \pm 0.19 & 3.29 \pm 1.94 & 1.48 \pm 0.40 & 1.65 \pm 0.45 & 1.63 \pm 0.16 & 2.65 \pm 0.41 & 2.16 \pm 0.32 & 2.18 \pm 0.23 & 1.59 \pm 0.35 & 2.27 \pm 0.35\end{array}$ \begin{tabular}{l|l|l|l|l|l|l|l|l|l|l} 
Hmox 1 & $1.00 \pm 0.07$ & $1.31 \pm 0.13$ & $1.12 \pm 0.08$ & $1.28 \pm 0.17$ & $1.32 \pm 0.10$ & $1.62 \pm 0.13$ & $1.46 \pm 0.11$ & $1.80 \pm 0.16$ & $1.44 \pm 0.09$ & $1.60 \pm 0.15$
\end{tabular} \begin{tabular}{|l|l|l|l|l|l|l|l|l|l|}
\hline DM & MW \#1 & MW \#2 & MW \#3 & MW \#4 & MW \#5 & MW \#6 & MW \#7 & CNF \#1 & CNF \#2 \\
\hline
\end{tabular}

$$
84 \text { Day High Dose }
$$

\begin{tabular}{|l|l|l|l|l|l|l|l|l|l} 
DM & MW \#1 & MW \#2 & MW \#3 & MW \#4 & MW \#5 & MW \#6 & MW \#7 & CNF \#1 & CNF \#2 \\
\hline
\end{tabular}

Interleukin 6

Interleukin 1 beta

C-C motif chemokine ligand 2

C-C motif chemokine ligand 22

Chemokine (C-X-C motif) ligand

Secreted Phosophoprotein 1

Metallothionein 1

Metallothionein 2

Heme oxygenase 1

$\begin{array}{lllllllllllll}\text { Il-6 } & 1.00 \pm .21 & 4.94 \pm 2.93 & 1.11 \pm 0.12 & 2.97 \pm 1.37 & 1.29 \pm 0.18 & 2.19 \pm 0.35 & 1.71 \pm 0.07 & 1.75 \pm 0.21 & 1.86 \pm 0.25 & 1.61 \pm 0.20\end{array}$ $\begin{array}{lllllllllllll}\text { Il-1B } & 1.00 \pm 0.20 & 1.30 \pm 0.47 & 0.61 \pm 0.07 & 0.75 \pm 0.09 & 0.62 \pm 0.07 & 0.73 \pm 0.05 & 0.58 \pm 0.07 & 0.68 \pm 0.11 & 0.71 \pm 0.14 & 0.62 \pm 0.12\end{array}$ \begin{tabular}{ll|l|l|l|l|l|l|l|l|l|l|l|l}
$\mathrm{Cc} 2$ & $1.00 \pm 0.21$ & $9.03 \pm 5.43$ & $1.05 \pm 0.14$ & $4.24 \pm 1.65$ & $1.22 \pm 0.12$ & $3.34 \pm 0.54$ & $1.98 \pm 0.34$ & $2.11 \pm 0.49$ & $1.71 \pm 0.20$ & $1.84 \pm 0.25$ \\
\hline
\end{tabular} $\begin{array}{llllllllllll}\mathrm{Ccl} 22 & 1.00 \pm 0.15 & 1.77 \pm 0.18 & 1.35 \pm 0.17 & 1.46 \pm 0.40 & 1.61 \pm 0.30 & 2.35 \pm 0.20 & 1.96 \pm 0.12 & 1.91 \pm 0.22 & 1.73 \pm 0.12 & 1.74 \pm 0.16\end{array}$ \begin{tabular}{|l|l|l|l|l|l|l|l|l|l|l|l|}
$\mathrm{Cxcl} 2$ & $1.00 \pm 0.21$ & $3.14 \pm 0.76$ & $1.22 \pm 0.12$ & $4.64 \pm 2.12$ & $1.83 \pm 0.22$ & $4.26 \pm 0.44$ & $4.31 \pm 0.70$ & $4.89 \pm 1.41$ & $3.05 \pm 0.35$ & $2.89 \pm 0.34$
\end{tabular} $\begin{array}{lllllllllllllll}\text { Spp1 } & 1.00 \pm 0.11 & 3.56 \pm .39 & 1.77 \pm 0.20 & 2.37 \pm 0.67 & 2.34 \pm 0.42 & 12.77 \pm 2.19 & 5.86 \pm 1.35 & 6.79 \pm 1.97 & 4.43 \pm 0.64 & 4.12 \pm 0.79\end{array}$ \begin{tabular}{l|l|l|l|l|l|l|l|l|l|l|l} 
Mt1 & $1.00 \pm 0.11$ & $0.90 \pm 0.12$ & $1.03 \pm 0.10$ & $0.70 \pm 0.20$ & $1.12 \pm 0.07$ & 1.210 .05 & $1.42 \pm 0.21$ & $1.36 \pm 0.14$ & $1.16 \pm 0.09$ & $1.18 \pm 0.05$
\end{tabular} \begin{tabular}{llllll|l|l|l|l|l|l|l} 
Mt2 & $1.00 \pm 0.11$ & $3.90 \pm 2.40$ & $1.25 \pm 1.07$ & $1.07 \pm 0.62$ & $3.42 \pm 2.26$ & $1.36 \pm 0.05$ & $1.94 \pm 0.37$ & $2.11 \pm 0.55$ & $1.49 \pm 0.09$ & $13.25 \pm 7.45$
\end{tabular} $\begin{array}{lllllllllllll}\text { Hmox } 1 & 1.00 \pm 0.08 & 1.21 \pm 0.16 & 0.76 \pm 0.08 & 0.90 \pm 0.10 & 0.88 \pm 0.04 & 1.20 \pm 0.03 & 1.17 \pm 0.08 & 1.04 \pm 0.11 & 0.93 \pm 0.07 & 1.05 \pm 0.09\end{array}$
Interleukin 6

Interleukin 1 beta

C-C motif chemokine ligand 2

C-C motif chemokine ligand 22

Chemokine (C-X-C motif) ligand :

Secreted Phosophoprotein 1
84 Day Low Dose

\begin{tabular}{|l|l|l|l|l|l|l|l|l|l}
\hline DM & MW \#1 & MW \#2 & MW \#3 & MW \#4 & MW \#5 & MW \#6 & MW \#7 & CNF \#1 & CNF \#2 \\
\hline
\end{tabular} $\begin{array}{llllllllllll}\text { Il-6 } & 1.00 \pm 0.141 & 1.17 \pm 0.14 & 1.29 \pm 0.14 & 1.11 \pm 0.10 & 1.02 \pm 0.08 & 1.12 \pm 0.27 & 1.05 \pm 0.10 & 1.37 \pm 0.25 & 0.96 \pm 0.06 & 0.80 \pm 0.11\end{array}$ \begin{tabular}{|l|l|l|l|l|l|l|l|l|l|l|l} 
Il-1B & $1.00 \pm 0.12$ & $0.79 \pm 0.06$ & $1.23 \pm 0.21$ & $1.16 \pm 0.28$ & $1.29 \pm 0.39$ & $1.15 \pm 0.16$ & $0.78 \pm 0.09$ & $0.86 \pm 0.21$ & $0.82 \pm 0.12$ & $0.67 \pm 0.06$
\end{tabular} $\begin{array}{llllllllllllll}\mathrm{Ccl} 2 & 1.00 \pm 0.10 & 0.96 \pm 0.10 & 0.88 \pm 0.07 & 0.95 \pm 0.17 & 0.74 \pm 0.05 & 0.87 \pm 0.09 & 1.02 \pm 0.09 & 1.03 \pm 0.04 & 0.72 \pm 0.05 & 0.76 \pm 0.07\end{array}$ \begin{tabular}{l|l|l|l|l|l|l|l|l|l|l|l|l|}
$\mathrm{Ccl} 22$ & $1.00 \pm 0.17$ & $0.94 \pm 0.14$ & $1.01 \pm 0.10$ & $0.93 \pm 0.16$ & $0.90 \pm 0.06$ & $1.16 \pm 0.13$ & $1.25 \pm 0.08$ & $1.37 \pm 0.07$ & $0.91 \pm 0.05$ & $0.93 \pm 0.08$ \\
\hline
\end{tabular} \begin{tabular}{l|l|l|l|l|l|l|l|l|l|l|l}
$\mathrm{Cxcl} 2$ & $1.00 \pm 0.31$ & $2.73 \pm 0.51$ & $3.37 \pm 1.57$ & $2.08 \pm 0.18$ & $2.15 \pm 0.35$ & $2.01 \pm 0.37$ & $3.98 \pm 1.10$ & $5.84 \pm 1.24$ & $3.52 \pm 0.40$ & $1.99 \pm 0.66$
\end{tabular} $\begin{array}{llllllllllllll}\text { Spp1 } & 1.00 \pm 0.07 & 0.95 \pm 0.16 & 1.27 \pm 0.12 & 0.98 \pm 0.09 & 1.31 \pm 0.19 & 1.16 \pm 0.15 & 1.83 \pm 0.25 & 1.54 \pm 0.15 & 0.82 \pm 0.15 & 1.24 \pm 0.10\end{array}$ 


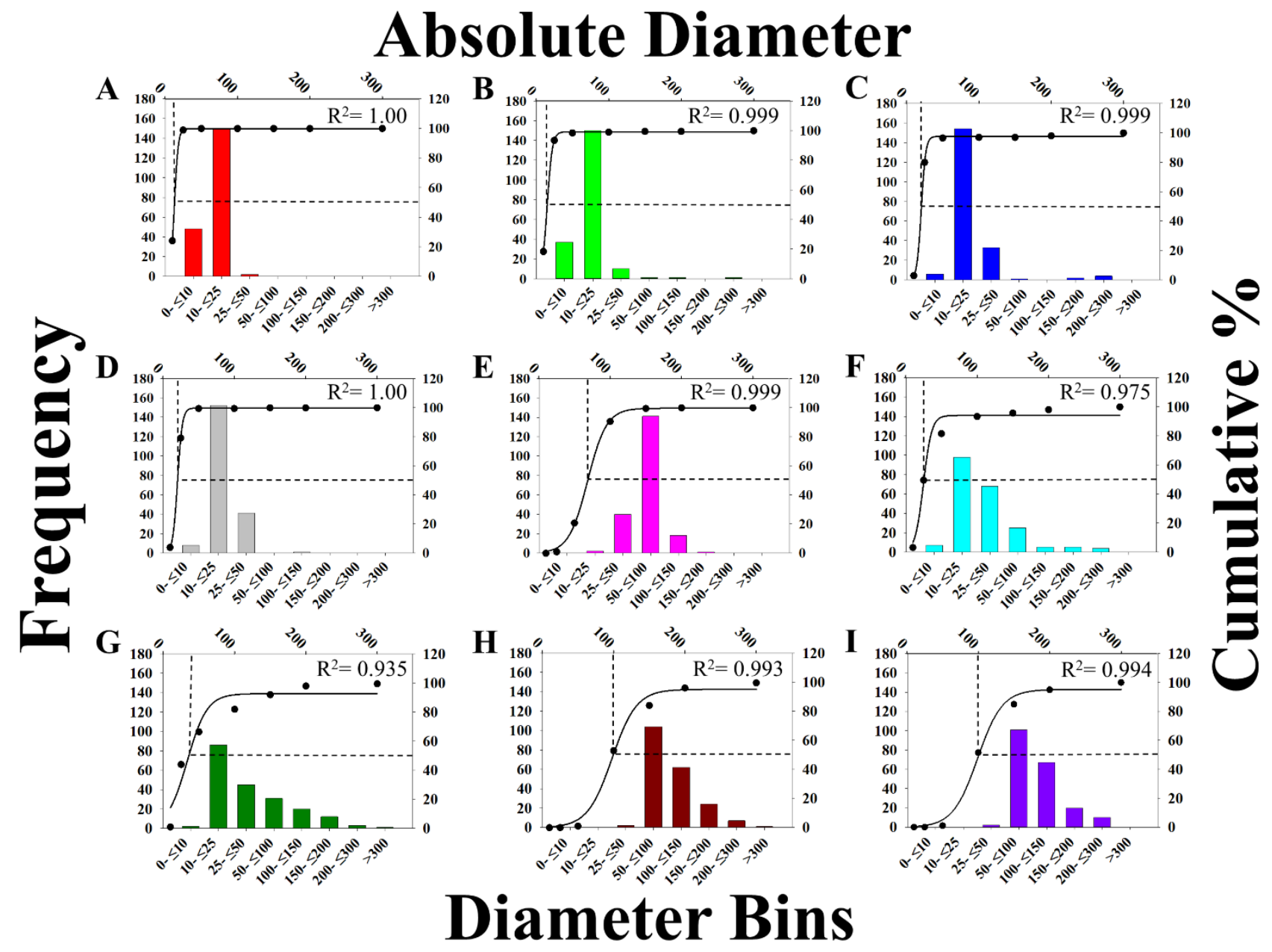

$\mathrm{MW} \# 1 \backsim \mathrm{MW} \# 2$ MW \#3

MW \#4 MW \#5 MW \#6

MW \#7

CNF \#1

CNF \#2

Supplementary Figure 3.1. Distributions of CNT/F diameter. Particles were binned according to size along the lower $\mathrm{x}$-axis with frequency on the left $\mathrm{y}$-axis. Additionally, percentage of accumulation is graphed on the right $y$-axis with the absolute diameter along the upper $x$-axis. The overlay line was 3 parameter sigmoidal curve of best fit with the point of 50\% accumulation indicated with dotted lines. Sizes are for particles in isopropanol suspension. 


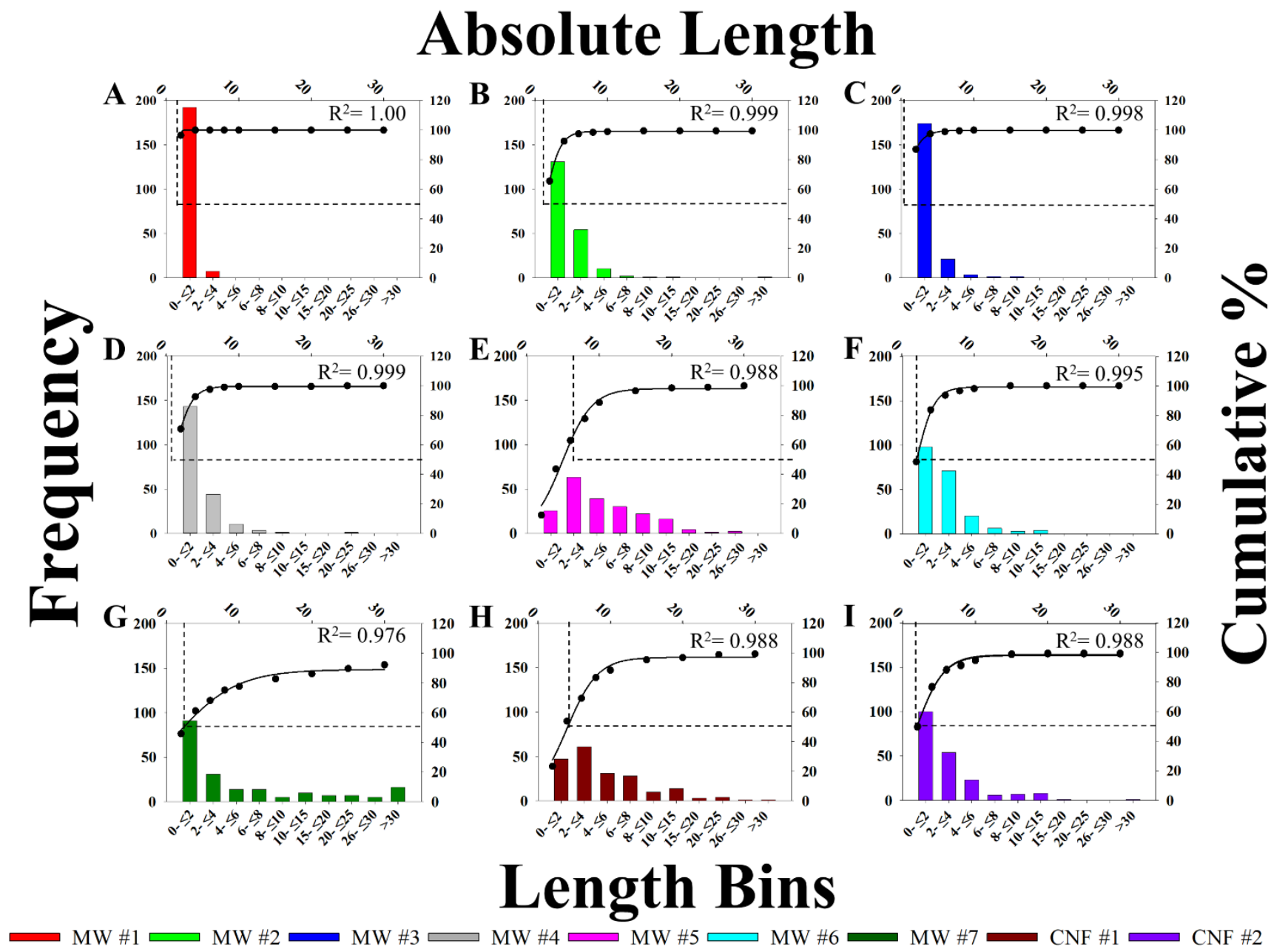

Supplementary Figure 3.2. Distributions of CNT/F length. Particles were binned according to size along the lower x-axis with frequency on the left y-axis. Additionally, percentage of accumulation is graphed on the right $y$-axis with absolute length along the upper $x$-axis. The overlay line is 3 parameter sigmoidal curve of best fit with the point of 50\% accumulation indicated with dotted lines. Sizing was for particles in isopropanol suspension. 

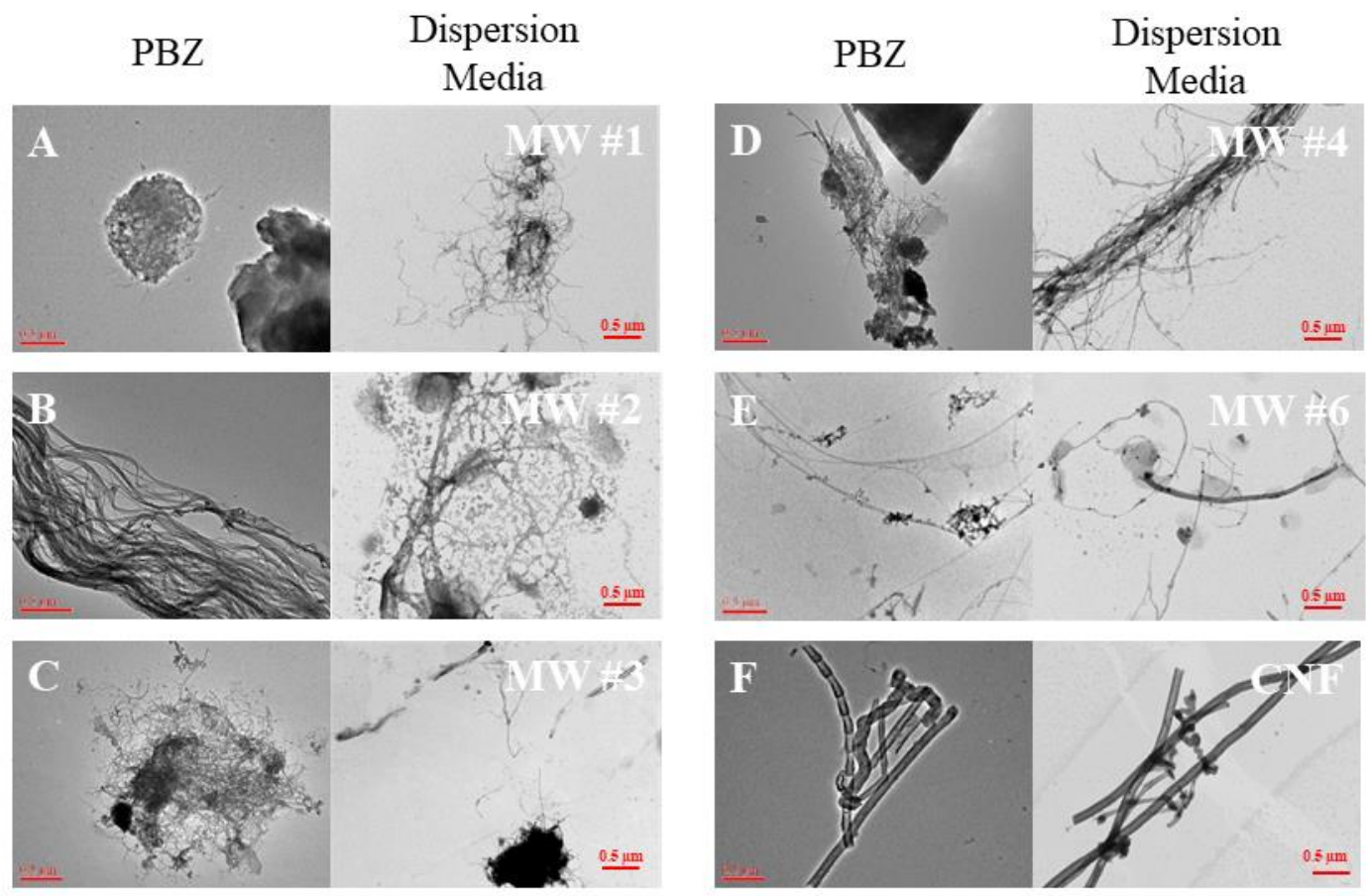

Supplementary Figure 3.3. Representative micrographs of CNT/F collected from personal breathing zone samples (PBZ) compared to particle dispersion in dosing media (DM) used for in vivo and in vitro dosing in the current study. Particle in DM retained similar profiles of particle size and agglomeration compared to PBZs to ensure proper representation of human occupational exposures. 


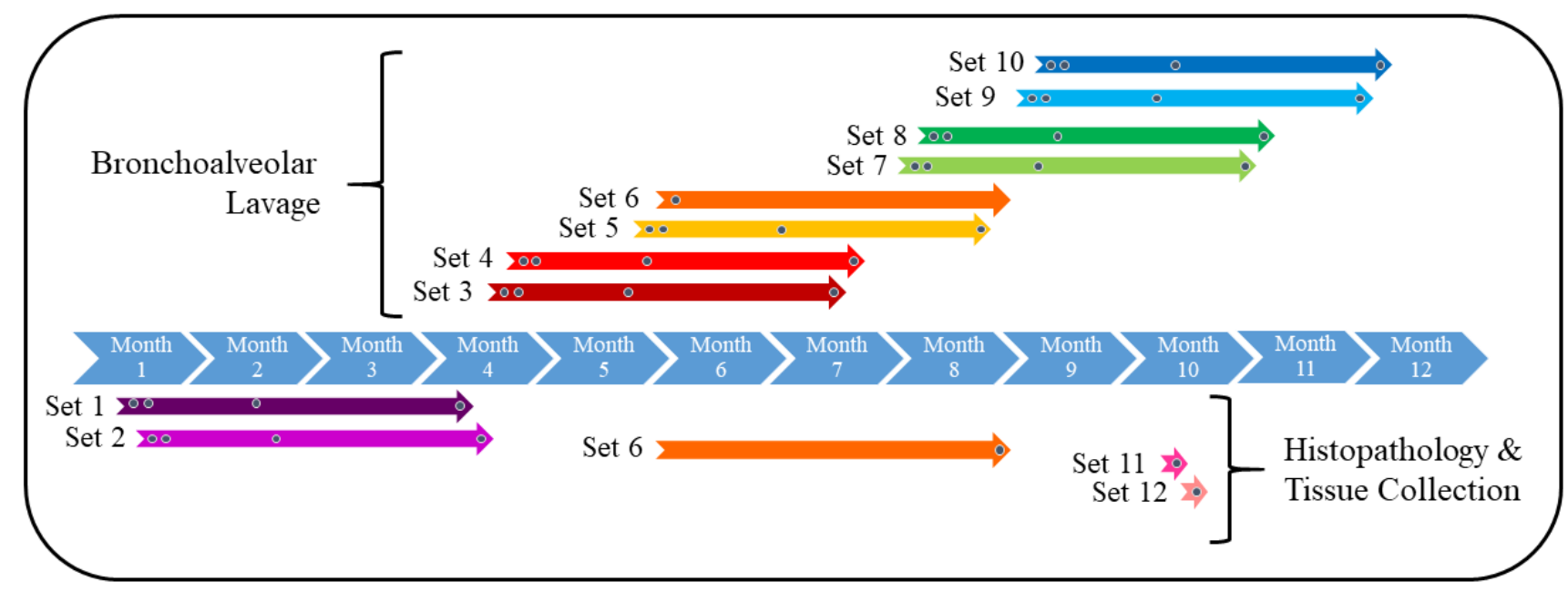

Supplementary Figure 3.4. Schematic of the mouse dosing and euthanasia schedule illustrating the timing of each rodent group across a year of time and distinguishing the bronchoalveolar lavage groups from the histopathology and tissue collection groups. 


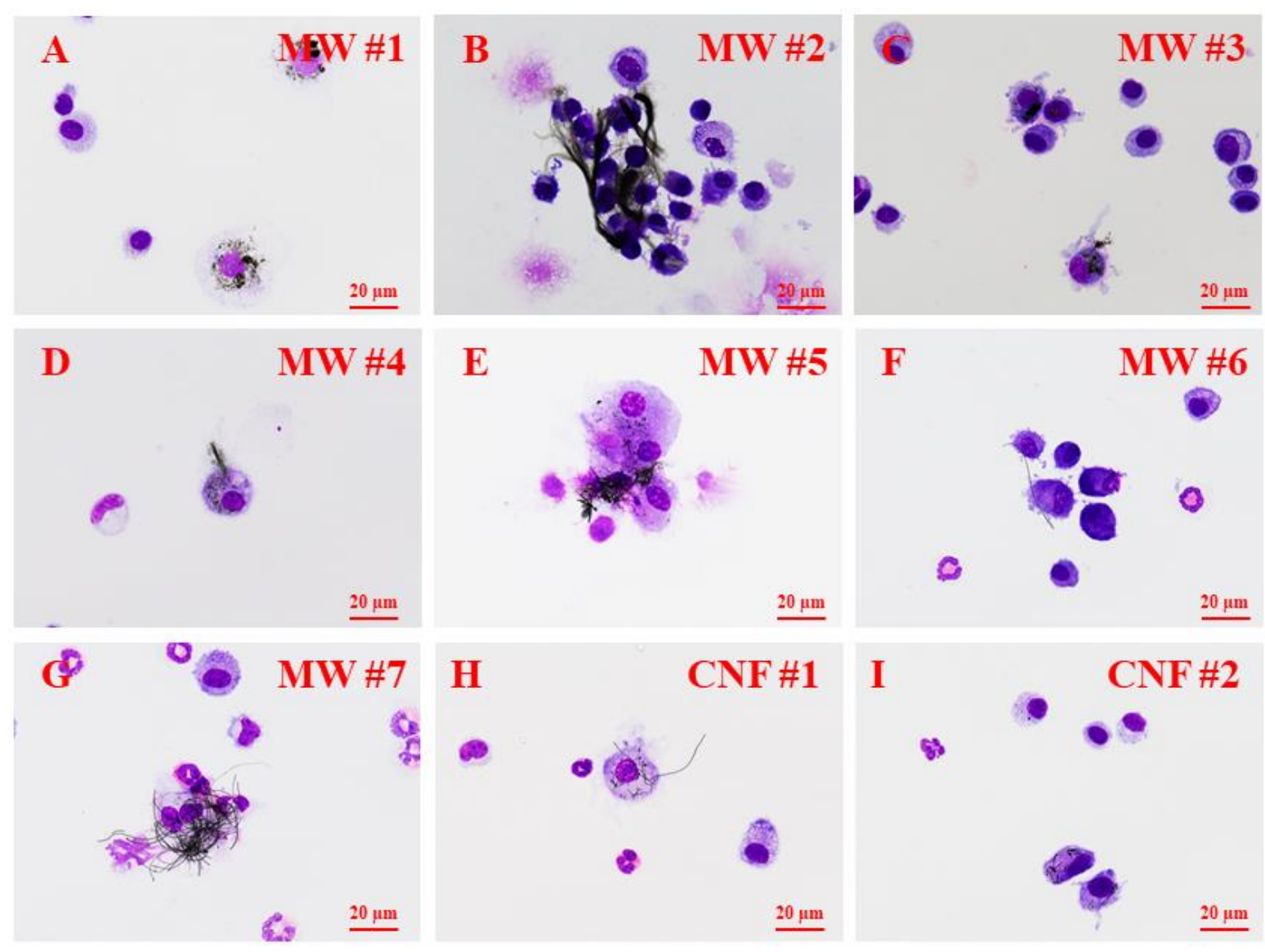

Supplementary Figure 3.5. Representative micrographs of BALF macrophages at 1 d post-exposure. 
A Day Post- Exposure

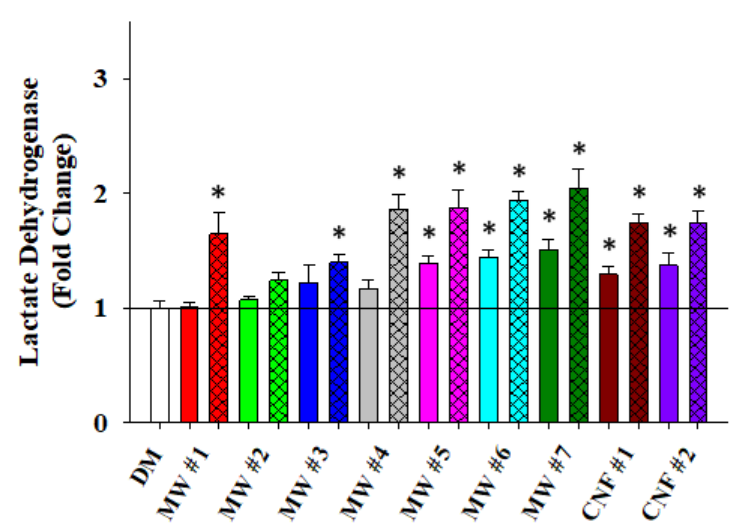

$\mathrm{B}$

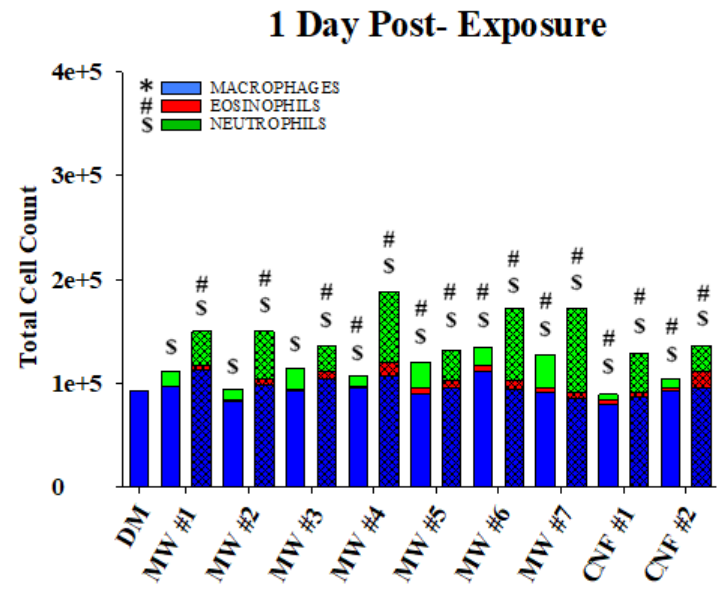

Supplementary Figure 3.6. Lactate dehydrogenase and inflammatory cell infiltration at 1- day postexposure to $\mathrm{CNT} / \mathrm{F}$ 


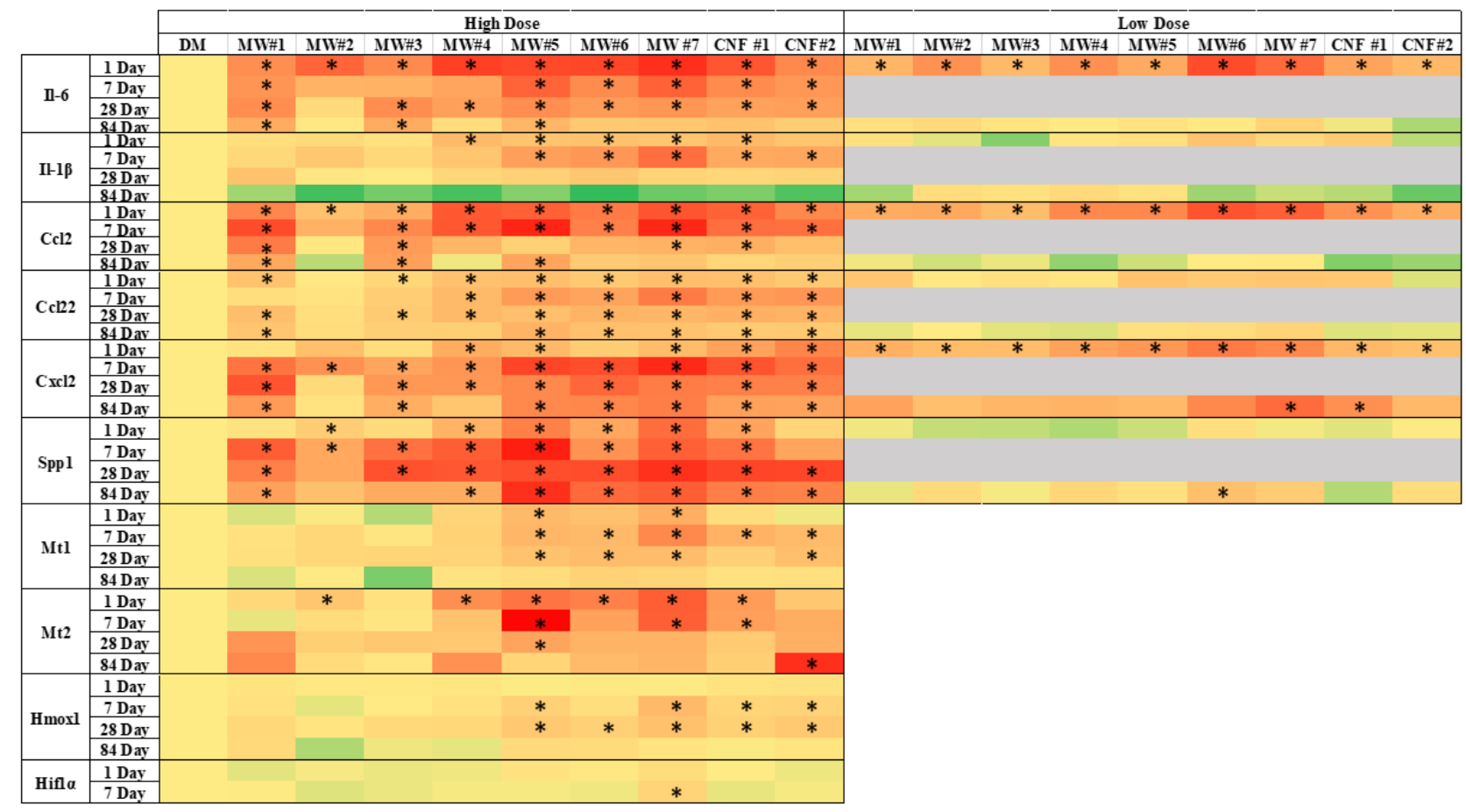

Supplementary Figure 3.7. Changes in mRNA expression of inflammatory cytokines and markers of oxidative stress from lung tissue at all four time points expressed as a heat map 

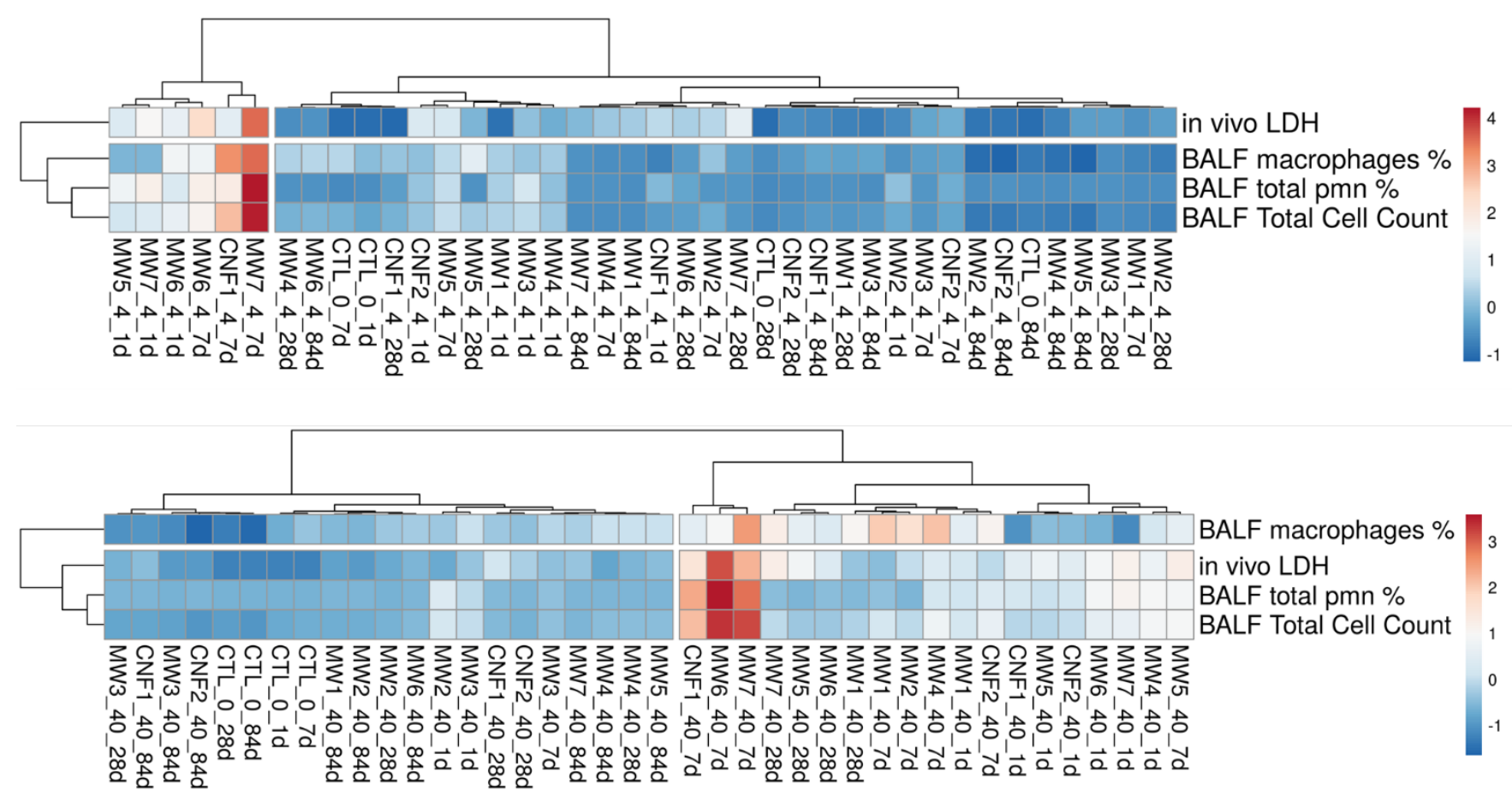

Supplementary Figure 3.8. Hierarchical clustering analysis comparing inflammatory outcomes from bronchoalveolar lavage fluid at all four times points. 

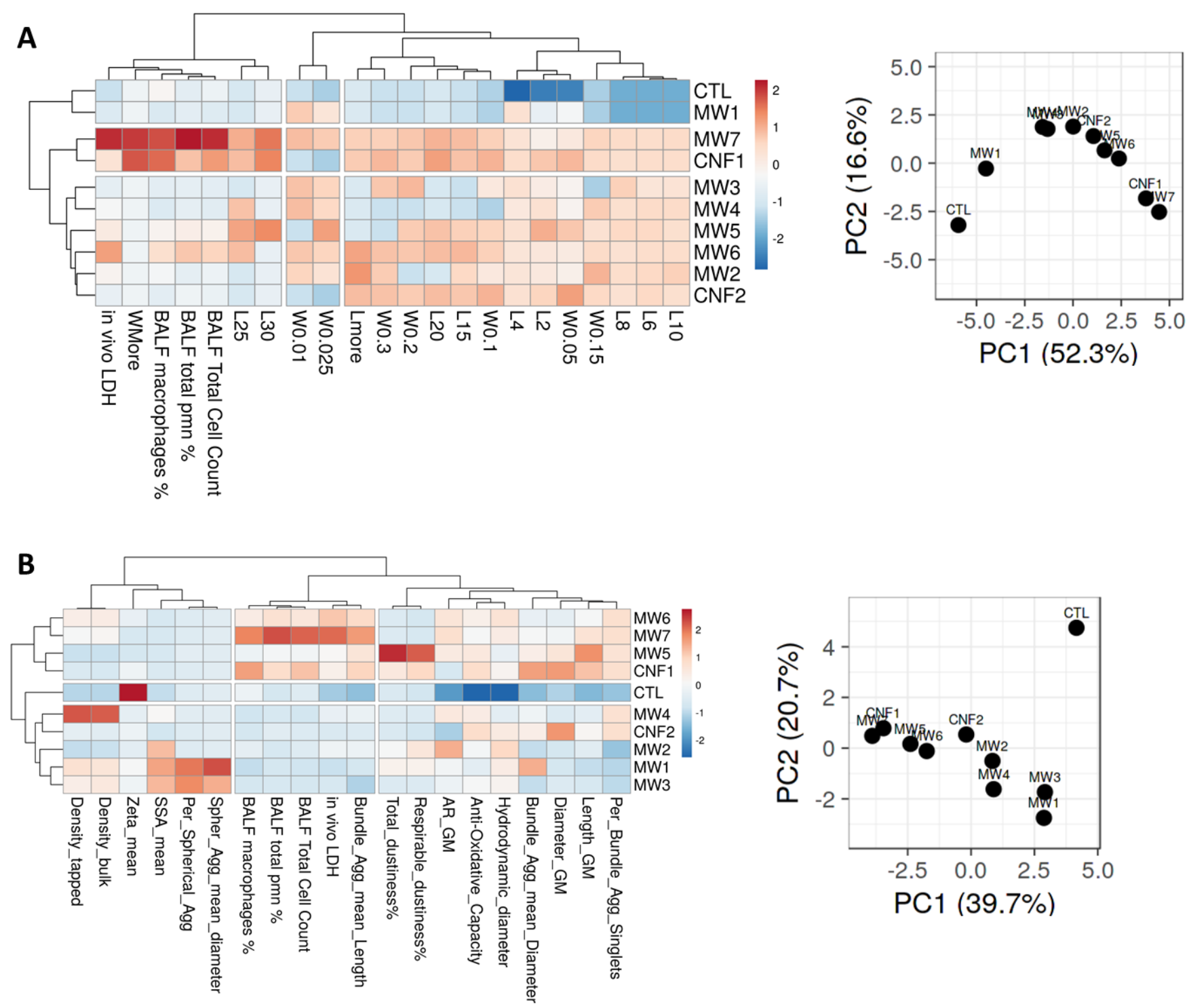

Supplementary Figure 3.8. Hierarchical clustering analysis (HCA) and principal component analysis (PCA) comparing inflammatory outcomes from bronchoalveolar lavage fluid with length-width L-W) and 'means only' of physicochemical characterization 

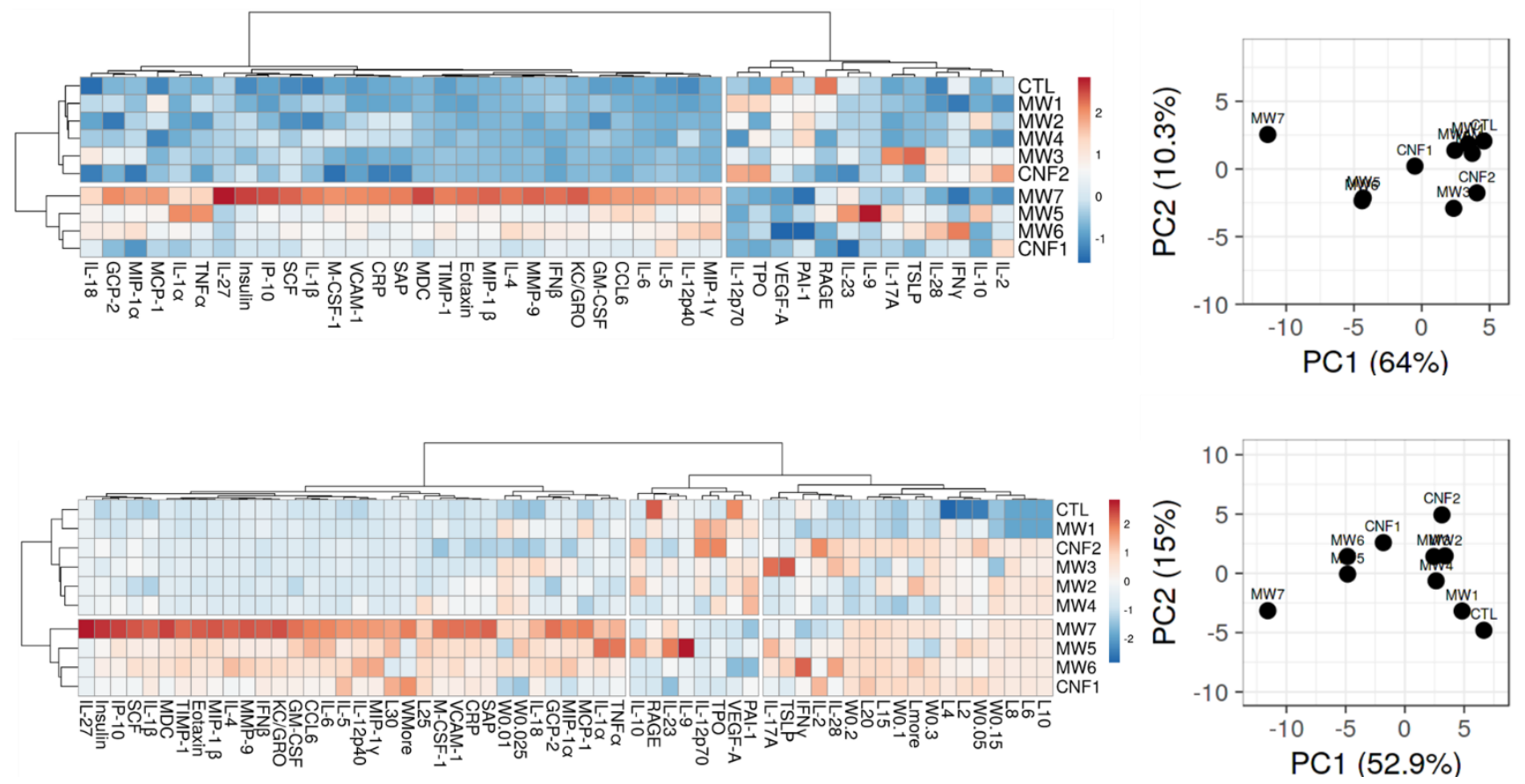

Supplementary Figure 3.9. Hierarchical clustering analysis (HCA) and principal component analysis (PCA) comparing proteins from bronchoalveolar lavage fluid alone and with length-width (L-W) physicochemical characterization parameters. 


\section{CHAPTER 4:}

Histopathology, Extra-pulmonary Translocation, and their Respective Physicochemical Drivers of the Broad Class of Carbon Nanotubes and Nanofibers Used or Produced in U.S. Facilities.

Kelly Fraser ${ }^{1,2}$, Ann Hubbs ${ }^{1}$, Robert M. Mercer ${ }^{1}$, Marlene Orandle ${ }^{1}$, Naveena Yanamala ${ }^{1}$, Lori Battelli ${ }^{1}$, Todd A. Stueckle ${ }^{1}$, Jake Jensen ${ }^{1}$, Tracy Eye ${ }^{1}$, John Hubczak ${ }^{1}$, Kara Fluharty ${ }^{1}$, Tiana Dodd ${ }^{1}$, Gary Casuccio $^{3}$, Kristin Bunker ${ }^{3}$, Traci L. Lersch ${ }^{3}$, Michael L. Kashon ${ }^{1}$, Sherri Friend ${ }^{1}$, Matthew Dahm ${ }^{4}$, Mary K. Schubauer-Berigan ${ }^{4,5}$, Vamsi Kodali ${ }^{1,2}$, Aaron Erdely ${ }^{1,2}$

${ }^{1}$ Health Effect Laboratory Division, National Institute for Occupational Safety and Health, Morgantown, WV; ${ }^{2}$ West Virginia University, Morgantown, WV; ${ }^{3}$ RJ Lee Group, Monroeville, PA; ${ }^{4}$ Division of Field Studies Evaluation, National Institute for Occupational Safety and Health, Cincinnati, OH; ${ }^{5}$ International Agency for Research on Cancer, Lyon, France 


\subsection{Abstract:}

Background: Carbon nanotubes and nanofibers (CNT/F) have been previously investigated for their potential toxicities, however comparative studies of the broad material class, especially those with a larger diameter, are lacking. Additionally, computational modeling correlating physicochemical characteristics and toxicity outcomes have been infrequently employed, and it is unclear if all CNT/F confer similar toxicity, including histopathology changes, pulmonary fibrosis, and extra-pulmonary translocation. Male C57BL/6 mice were exposed to $40 \mu \mathrm{g}$ of one of nine CNT/F (MW \#1-7 and CNF \#1-2) commonly found in exposure assessment studies of U.S. facilities with diameters ranging from 6-150 nm. Human fibroblasts $(0-20 \mu \mathrm{g} / \mathrm{ml})$ were used to assess the predictive value of in vitro to in vivo modeling systems.

Results: All materials induced histopathology changes, though specific outcomes were variable in severity and distribution. In general, MW \#5-7 and CNF \#1 induced greater histopathology changes compared to MW \#1 and \#3 while MW \#4 and CNF \#2 were intermediate in effect. Differences in individual alveolar or bronchiolar outcomes and severity correlated to physicochemical characteristics of nominal tube physical dimensions and how the materials agglomerated. Human fibroblast monocultures were found to be insufficient to fully replicate in vivo fibrosis outcomes suggesting the need for more advanced cell culture in vitro models. Transport of the $\mathrm{CNT} / \mathrm{F}$ to the tracheobronchial lymph nodes was present for any material with significant alveolar deposition. Singlet accumulation in the lung at day 1 provided predictive power for liver accumulation $84 \mathrm{~d}$ post-exposure. Pleural penetrations were observed more consistently in $\mathrm{CNT} / \mathrm{F}$ with larger lengths and diameters.

Conclusion: Physicochemical characteristics, notably nominal tube dimension and agglomerate size, were predictive of histopathology and were essential for grouping of materials. Particles of greater nominal tube length were generally associated with increased severity of histopathology outcomes. Larger particle lengths and agglomerates were associated with more severer bronchi-bronchiolar outcomes. Spherical agglomerated particles of smaller nominal tube dimension were linked to granulomatous inflammation while a mixture of smaller and larger dimensional CNT/F resulted in more severe alveolar injury. Extrapulmonary translocation was also associated with particle size, suggesting that particles of moderate length and diameter with minimal agglomeration were more likely to translocate to the liver while particles of all sizes were observed in the tracheobronchial lymph nodes. Notably, singlet lung burden at $1 \mathrm{~d}$ postexposure correlated with liver burden at $84 \mathrm{~d}$ post-exposure. 


\subsection{Introduction:}

Recommended exposure limits (REL) established by NIOSH take into consideration two key factors when determining the quantitative risk: the severity and likelihood of adverse effects upon exposure to a hazardous material during a 45 year working lifetime. For carbon nanotubes and nanofibers, the REL has historically been established primarily upon the fibrogenic and inflammatory capabilities of these materials and relied upon short term and subacute animal studies that investigated pulmonary inflammation, granulomatous response, thickening of the alveolar septum, and extrapulmonary translocation [57]. From these studies, human exposure equivalents can be predicted, thus allowing for the generation of the recommended exposure limit.

Due to lack of carcinogenicity studies and the difficulties in predicting long term effects from acute inflammation studies, histopathological changes following CNT exposure have been critical to establishing occupation exposure limits. These changes, such as fibrosis, are critical as they represent fundamental and potentially irreversible changes in the lung $[9,10,51,56,116]$. However, to date, it is in unclear if all CNT/F confer the same histopathology changes in the lung. Furthermore, systemic effects and systemic translocation have been minimally investigated due to difficulties in monitoring particle behavior and translocation post-exposure $[59,60,66]$.

Due to the broad differences in physicochemical characteristics of CNT/F including agglomeration, length, diameter, and respirability, extrapolations and predictive modeling have been severely limited. This study aims to generate an accurate and effective safety profile for a series of MWCNT/F of various physicochemical characteristics. Specifically, in this portion of the study, Part III, an in-depth evaluation of histopathology and systemic translocation was completed to assess differences in a particle's ability to induce long term structural changes in the lung as well as the potential for adverse systemic effects. 


\subsection{Results and Discussion:}

The nine materials used in this study were selected to represent the broad class of CNT/F. In brief, seven MWCNT and two CNF were ordered according to their diameter as reported by the production facility and are referred to as MW \#1-7 and CNF \#1-2 (Figure 1). MWCNT-7/Mitsui-7, labeled as MW \#5 in this study, was used as a benchmark material for comparison. Analyses and interpretation of detailed physicochemical characteristics and genotoxicity [182] were presented separately. In this section of the evaluation of $\mathrm{CNT} / \mathrm{F}$ toxicities, in vivo assessments for pathological changes and translocation were compared and correlated to physicochemical characteristics. Male C57BL/6J mice were exposed to $40 \mu \mathrm{g}$ of $\mathrm{CNT} / \mathrm{F}$ or DM via oropharyngeal aspiration and euthanized at 1 and $84 \mathrm{~d}$ post-exposure. Historically, a $40 \mu \mathrm{g}$ dose of $\mathrm{CNT} / \mathrm{F}$ was sufficient to induce pathological changes within an $84 \mathrm{~d}$ post-exposure time point and necessary to induce significant alveolar fibrosis $[9,10,19,51]$. Liver, tracheobronchial lymph nodes, and right lungs were fixed with formalin with the lungs being gravity filled to preserve inflation to evaluate particle distribution, alveolar fibrosis, and other histopathology changes.

Additional assessments in vitro were used for comparison and to further investigate the mechanisms by which the pathological changes occurred. For comparison, human fibroblast cells were cultured and exposed to $0-20 \mu \mathrm{g} / \mathrm{ml}$ of each of the nine CNT/F for 24 hours and subsequently assessed for cell viability and proliferation as well as collagen- $1, \alpha$-smooth muscle actin, and TGF- $\beta$ production.

\subsubsection{Particle characterization}

The CNT/F used in this study were extensively characterized in a previous publication and can be found in the supplementary data (Supplementary Tables 1-3, Supplementary Figures 1-2) [182]. Representative micrographs from transmission electron microscopy (TEM) images of each particle are shown in Figure 1. It was quite evident that as CNT/F increase in nominal tube diameter and length, a general decrease in bundled agglomeration was observed with a transition point around MW \#4. MW \#1 and \#3 were predominately spherical agglomerates with a geometric mean under $1 \mu \mathrm{m}$ (Supplemental 
Table 2). MW \#2 was a unique high entangled MWCNT as previously described $[19,182]$ that forms two separate populations of extensive 'rivers' of aggregates, and agglomerates of aggregates, in the micron size range (Figure 1; Supplemental Table 2). MW \#4-7 and CNF \#1-2 were almost exclusively bundled agglomerates exceeding a 3:1 aspect ratio $(\geq 96 \%)$ or as singlets.

\subsubsection{Histopathology}

For comparative potency between materials, $40 \mu \mathrm{g} \mathrm{CNT} / \mathrm{F}$ is a known administered dose to induce pathology including alveolar fibrosis. Previously we have indicated this dose to likely exceed a lifetime exposure at average exposure levels in U.S. facilities $[68,164]$. The goal of this study was less concerned with deposited dose with relation to human equivalency as compared to relative potency. Combined with the known fact that MW \#5 (Mitsui-7/MWCNT-7) does not induce a significant alveolar fibrosis up to 20 $\mu \mathrm{g}$, the dose of $40 \mu \mathrm{g}$ was selected to be able to evaluate differences of effect between the nine CNT/F [9, 10]. A representative micrograph of healthy control tissue can be found in Figure 2A.

At 84 d post-exposure, CNT/F often caused granulomatous inflammation, defined as an organized infiltrate of epithelioid macrophages which may form giant cells and are often admixed with lymphocytes, plasma cells, and fibrosis. Diagnoses within this category included granulomatous bronchopneumonia, but also included histiocytic bronchopneumonia, granulomatous bronchointerstitial pneumonia, lymphogranulomatous interstitial pneumonia, and granulomatous alveolitis. An example image can be found in Figure 2B. The distribution and severity of the granulomatous response was significant among all treatment groups (Table 1).

Morphologic responses to $\mathrm{CNT} / \mathrm{F}$ sometimes included proliferative changes within airways that sometimes obstructed the lumen bronchioles and alveolar ducts. These were classified as proliferative bronchiolitis obliterans (Figure 2C) when bronchioles were affected. If the alveolar ducts were involved, these were classified as bronchiolitis obliterans-like lesions of the alveolar duct. These potentially 
obstructive lesions often formed in association with granulomatous inflammation and projected into the lumen of bronchioles and alveolar ducts. MW \#1, 5 and CNF \#2 did not induce proliferative bronchiolitis obliterans or bronchiolitis obliterans-like morphologic alterations and the incidence was only 1/5 for MW \#3 (Table 1). MW \#4 had incidence in half the analyzed lungs, but significance was not reached in terms of severity and distribution. MW \#2, 6, 7, and CNF \#1 induced significant bronchiolitis obliterans-like changes (Table 1). We have previously shown proliferative bronchiolitis obliterans lesions to occur following MW \#2 exposure consistent with the large aggregates [19].

In the alveolar region of the lungs, mice exposed to CNT/F often had accumulations of alveolar macrophages, a finding also known as alveolar histiocytosis (Figure 2D). In some cases, alveoli with alveolar macrophage accumulation also showed evidence of a tissue response (histiocytic alveolitis) and/or neutrophils accompanied the macrophages (histiocytic and neutrophilic alveolitis). Because the responses represented a spectrum of predominantly histiocytic responses, these diagnoses were grouped together in the summary table. All CNT/F induced alveolar histiocytosis except for MW \#2 (Table 1), the CNT with larger aggregates and agglomerates that has less alveolar deposition compared to other CNT/F [19].

Exposed mice also developed hypertrophy of the bronchiolar epithelium as well as alveolar epithelial hypertrophy and hyperplasia (Table 1; Figure 2E-F). Particle deposition and persistence were necessary for bronchiolar hypertrophy and alveolar hypertrophy and hyperplasia. MW \#1 and \#3 did not induce bronchiolar epithelial hypertrophy while MW \#2 did not induce alveolar epithelial hypertrophy and hyperplasia. MW \#4-7 and CNF \#1-2 had significant effects for both.

Additional morphologic changes regarding lymphatics are of note. The lymphatics of the lung control interstitial fluid balance, transport cells of the immune system, and participate in particle clearance in the lung [183-185]. In this study, particle accumulation, particularly in materials of longer length, could be found at the bronchoalveolar junction, the location of some of the smallest lymphatic vessels (Figure 
$2 \mathrm{G})$. This accumulation has the potential to obstruct lymphatic flow and may be a mechanism for decreased clearance in fibrotic and inflamed airways. Similarly, needle-like particles can damage macrophages attempting to reach the areas of smaller lymphatic flow surrounding the terminal bronchioles resulting in further particle accumulation. Even when unobstructed by previous particle deposition, clearance of long, somewhat rigid particles may be difficult in the narrow and curving lymphatic pathways. Ectasia, or dilation, of lymphatic was observed as was also previously reported following MWCNT exposure [51]. It remains to be determined whether pleural accumulation of CNT/F and the airway fibrosis were attributable to altered lymphatic clearance, if airway fibrosis attributed to altered particle clearance, or both. However, in some cases clusters of CNT/F could be seen extending from dilated lymphatic through the airway wall to the surface of airways suggest lymphatic obstruction and potential release of CNT/F back into airways (Figure 2G). Furthermore, lymphatics are not restricted to the pulmonary lobule, but also present in interlobular septa and the pleura [185]. Macrophages containing particles often traffic to the lymphatics to clear particles from the lung. Additionally, macrophagemediated transport of $\mathrm{CNT} / \mathrm{F}$ to pleural lymphatics can potentially release $\mathrm{CNT} / \mathrm{F}$ near the pleural lining if the particles are cytotoxic. At least more than one incidence of pleural penetration or accumulation at the pleural surface was seen following MW \#5-6 and CNF \#1-2 exposures, though more refined techniques such as enhanced darkfield microscopy may be necessary to definitively conclude that some, but not all particles were capable of pleural penetrations (Figure 2H). MW \#5 has been previously reported to penetrate the pleura, though it is unclear if all particles, particularly spherical agglomerates (e.g., MW \#1 and \#3) may penetrate the visceral pleura [51].

\subsubsection{Histopathologic assessment of pulmonary fibrosis}

Severity and distribution for two types of pulmonary fibrosis, bronchial / bronchiolar and alveolar, was determined (Table 1, Figure 2I-J). Bronchial and bronchiolar fibrosis occurred frequently in 
conjunction with bronchiolitis obliterans and/or granulomatous inflammation. Incidence of $100 \%$ was observed for MW \#5-7 and CNF \#1. MW \#2 also had $100 \%$ incidence given the nature of the highly entangled particle that deposits significantly in the conducting airways [19]. MW \#1, \#3, and CNF \#2, although not without effect, had less severity and distribution. Histopathologic assessment of alveolar interstitial fibrosis indicated greater severity and distribution with MW \#4-7 and CNF \#1 as compared to other materials, especially MW \#3 and CNF \#2 (Table 1).

\subsubsection{Morphometric analysis of pulmonary fibrosis}

To further investigate pulmonary fibrosis, bronchiolar and alveolar fibrosis was quantitatively evaluated by morphometry. To measure fibrosis in the terminal bronchioles, photomicrographs were taken of trichrome-stained lung sections. Using ImageJ, the area of bronchiolar fibrosis, the total area of the region, and the length of the basement membrane were measured. This enabled calculation of the area of fibrosis per micron of basement membrane (Figure 3) and as percent area of fibrosis per area of the total field of view (Supplemental Figure 3). Two distinct regions were systematically sampled in each lung section: 1) areas of minimal to no severity, or least affected bronchioles, and 2) areas of most severe fibrosis, or most affected bronchioles. In the least affected areas, no significant differences were seen from control (Figure 3A). For the most affected regions, a significant increase in bronchiolar fibrosis was measured in MW \#2, 4-7 and CNF \#1 exposed lung tissue, but not for MW \#1, 3, or CNF \#2 (Figure 3B). Fibrosis was consistent with areas of prominent particle deposition, particularly areas with particle agglomerates. Similar findings were reported by Duke et al. in 2018 in which they quantified the area to perimeter ratio of airway fibrosis and found that "rod-like" MWCNT that are similar in morphology to MW \#5-7 and CNF \#1-2 of the present study were more likely to induce greater airway fibrosis than “tangled" MWCNT similar to MW \# 1 and \#3 in the present study [124]. These differences in airway fibrosis were attributed to variability in clearance mechanisms and the translocation of "rod-like" particles 
across the epithelium where pathways driving fibroblast activity can be initiated. Interestingly, CNF \#2, while having a diameter more like MW \#5-7 and CNF \#1, did not induce significant bronchiolar fibrosis as measured by morphometry. This may be due to the smaller nominal tube lengths (Supplemental Table 1; Supplemental Figure 2). These findings support the conclusion that the physical presence of particle deposition in airways, with physical dimensions of greater diameter and length, act as a potent driver of airway fibrosis.

Alveolar fibrosis is a well-documented outcome following CNT exposure [9, 10, 51]. Morphometric point and intercept counting was used to measure the thickness of alveolar fibrillary collagen at 84 d post-exposure. Fibrillary collagen was significantly increased in mice exposed to MW \#5-7 and CNF \#1 (Figure 4A). Representative micrographs of vehicle exposed as well as MW \# 3, 6, and CNF \#1 can be found in Figure 4B-E respectively. This fibrosis was also related to the presence of particle in the interstitium. The increased alveolar fibrosis of MW \#5 exposed lungs was consistent with previous investigations [9, 10]. Increases were found for MW \#1, MW \#4, and CNF \#2 but significance was not achieved, suggesting a greater than $40 \mu \mathrm{g}$ delivered dose would be necessary to induce significant alveolar fibrosis. Mercer et al., compared single-walled (SW)CNT and MWCNT rodent lung exposures and reported that macrophages were less likely to recognize and phagocytose SWCNT compared to MWCNT, leading to greater interstitial accumulation and fibrosis [9]. As the size of MW \#1 in the current study approaches the size of SWCNT, it is suspected that the infrequent singlet or very small agglomerates may escape macrophage recognition permitting translocation to the alveolar interstitium and induction of interstitial fibrosis, therefore behaving in a similar fashion to SWCNT resulting in the mild but not significant increase in interstitial fibrosis. There was no effect for MW \#2 or \#3. This was expected for MW \#2 given the sample is less likely to accumulate in the alveolar region [19]. Of note, several instances were observed, particularly in MW \#3 exposed lung tissue, where small agglomerates of particles were 
present in the interstitium, but fibrosis was not present, suggesting that particle singlets were more likely to induce fibrosis than tangled agglomerated particles (Figure 4C).

4.3.5. Hierarchical clustering analysis (HCA) and principal component analysis (PCA) of the histopathology outcomes

HCA and PCA were performed to group CNT/F by adverse histopathology. Initially all outcomes assessed were analyzed (Figure 5). Overall, severity of response was greater for MW \#4-7 and CNF \#1 as compared to MW \#1-3 and CNF \#2 which grouped more closely with the sham group. These results were intriguing as the lesser responding grouping included a large nominal diameter material in CNF \#2 as well as MW \#2 which primarily has bronchiolar effects. To segregate regional effects, the alveolar and bronchiolar responses were evaluated separately. The bronchiolar effects were like the overall response except for MW \#2 which corresponded in response more closely with MW \#6 and \#7 (Figure 6A). MW \#1, \#3, and CNF \#2 localized with the sham exposed mice indicating minimal to no bronchiolar effects. The analysis to address specifically the alveolar outcomes indicated that MW\#5, \#7, and CNF \#1 had significant effects compared the remaining materials (Figure 6B). These three materials segregated out as they induced marked responses in all five alveolar assessments (Figure 5, Table 1). The other materials had variable significance in alveolar responses. MW \#2 and \#3 induced milder responses in general with the exception of granulomatous inflammation and MW \#4 and \#6 had generally stronger effects than MW \#2 and \#3 but less than MW \#5,\#7, and CNF \#1 (Figure 6B, Table 1). In summary MW \#5, \#7 and CNF \#1 overall induced the strongest with effects in both the bronchiolar and alveolar regions hence their close clustering in Figure 5. MW \#4 and MW \#6 were positive in effect for bronchiolar and alveolar effects but generally milder compared to MW \#5, \#7 and CNF \#1. MW \#1-3 and CNF \#2 generally induced milder or specific regional effects as was the case with $\mathrm{MW} \# 2$ in the bronchiolar region. 
4.3.6. HCA and PCA of the histopathology outcomes with physicochemical characteristics

The next step was to include the physicochemical characteristics to provide inference into specific characteristics that contributes to developing pathology. The analysis was done in three ways: 1) detailed characterization of binned length (L) and diameter/width (labeled as W for figure clarity for easier distinction from L); 2) standard physicochemical data using means only from Supplemental Tables 1-3; and 3) the combination of binned length and diameter/width and means only data. The data were generated for all pathology outcomes as well as bronchiolar and alveolar separately. Previously we have shown that the binned length and diameter provided the resolution to accurately group CNT/F in comparison to using means only [182]. In fact, the grouping using binned length and diameter alone was comparable to extensive characterization of all physicochemical parameters. For the histopathological outcomes in this study, adding the physicochemical characteristics did not exactly group as outcomes alone. Variations of MW \#1-4 were typically in the lower responding group with remaining materials in the more toxicity group (Figures 7-8; Supplemental Figures 4-9). The analysis of outcomes with physicochemical characteristics indicate that MW \#1 and \#3 consistently are of lower toxicity compared to MW \#5-7 and CNF \#1. MW \#4 and CNF \#2 represent a transition in toxicity from less to greater severity.

For alveolar pathology responses, in general all analyses highlighted associations with length, the larger length bins, and the agglomeration state (per_bundle_agglomerate_singlet, bundle_agglomerate_mean_length, bundled_agglomerate_mean_diameter). These results indicate that materials which contain nominal tubes with larger lengths, which were more prevalent for MW \#5, \#7, and CNF \#1 (Supplemental Figure 2) can induce more significant injury to the alveolar region. This was consistent with the material grouping for alveolar effects alone (Figure 6B) and with physical dimension binning (Figure 8B). The two-dimensional sizing was also a key factor in predicting alveolar pathology. MW \#1 and \#3 clearly form more spherical agglomerates (Supplemental Table 2). MW \#4 was the transition point switching from majority spherical agglomerates to bundled/elongated agglomerates. The 
switch from spherical agglomerates to more elongated agglomerates and singlets is driven by increasing physical size. Therefore, as increasing length corresponds more with general severity of alveolar pathology, association with the transition away from spherical agglomerates (e.g., MW \#1 and MW \#3) was consistent. The inclusion of binning of the physical dimensions was able to better separate granulomatous inflammation from the rest of the alveolar-associated pathology with an association of the L2, L4, and W0.05 bins (Figure 7 and 8B). As all materials induced granulomatous inflammation (Table 1), correspondence with the smaller length bins, something that was consistent for all samples (Supplemental Figure 2), were the associated drivers. Using means only (Supplemental Figure 7 and 9), the association of granulomatous inflammation and alveolar interstitial fibrosis did not provide associations with any physical dimensions of length or width.

For bronchiolar pathology responses, the primary physicochemical characteristics driving the 4 measured outcomes were bundle_agglomerate_mean_length as well as longer lengths and wider diameters (e.g., L25, L30, and W0.15). This indicates a combination of increasing length and width, with a larger bundle_agglomerate_mean_length (e.g., MW \#6 and \#7) conferred greater bronchiolar toxicity. The analysis of bronchiolar effects alone grouped MW \#1, MW \#3, and CNF \#2 with the sham group suggested a diminished response. This was intriguing given $\mathrm{CNF} \# 2$ is a larger diameter material. Looking in more detail at the physicochemical characteristics, $\mathrm{CNF} \# 2$, while having an increased diameter, did not have the corresponding increased length, especially in comparison to CNF \#1 (Supplemental Figure 1 and 2; Supplemental Table 1). This was further illustrated for the bundle_agglomerate_mean_length for CNF \#2 that was closer to MW \#1 than CNF \#1 (Supplemental Table 2). Overall, increasing length and diameter, corresponding to bundled agglomerate that are larger than a 3:1 ratio conferred greater bronchiolar toxicity which can be stratified as MW \#6 and MW \#7 > MW \#4, MW \#5, and CNF \#1 > MW \#1, MW \#3, and CNF \#2. We confirmed the conducting airway effects of MW \#2 as the larger of the two subpopulations of aggregates/agglomerates was too large to reach the alveolar space and had the largest 
bundle_agglomerate_mean_length. The L2, L4, and W0.05 bins did not correspond to any bronchiolar effects (Figure 7 and 8A), which was consistent with those factors primarily driving alveolar granulomatous inflammation.

\subsubsection{In vitro fibrosis assessments}

Human fibroblasts were used in this study to investigate the mechanisms driving fibrosis outcomes observed in vivo and to explore the efficiency of this in vitro system to model in vivo observations. The same nine $\mathrm{CNT} / \mathrm{F}$ were used at concentrations of $0-20 \mu \mathrm{g} / \mathrm{ml}$ ranging from occupationally-relevant to overtly high doses in order to observe the full range of potential effects. These concentrations correlate to roughly $0-100 \mu \mathrm{g}$ doses respectively in vivo for mice. Using a WST-1 assay, the percentage of cell viability and proliferation was quantified (Figure 9A). Within this dose range, minimal changes in cell viability were seen suggesting that doses up to $0.2 \mu \mathrm{g} / \mathrm{ml}$ did not induce cell death.

Fibroblasts are the key generators of collagen-1, the main collagen isoform comprising pulmonary fibrosis. Western blot was used to quantify collagen-1 production following $0.06 \mu \mathrm{g} / \mathrm{ml}$ exposure. Collagen-1 outputs were variable, though CNF \#1 did induce a significant increase (Figure 9B). Additionally, $\alpha$-smooth muscle actin was not significantly increased following $0.06 \mu \mathrm{g} / \mathrm{ml}$ particle exposure in any treatment group (Figure 9C). Previous studies have demonstrated the key role of TGF- $\beta$ as an upstream modulator of pulmonary fibrosis. TGF- $\beta$ was also variable between particle exposures and doses in a manner that did not always correlate to dose (Figure 9D). However, as a general trend, materials MW \#4-7 and CNF \#1-2 induced greater TGF- $\beta$ production.

4.3.8. Hierarchical clustering and PCA of the fibroblast outcomes with physicochemical characteristics

The initial analysis of the four fibroblast endpoints (Figure 10), cell viability with collagen, $\alpha$ SMA, and TGF $\beta$ protein levels, did not correspond to in vivo for all histopathological outcomes (Figure 5) or 
separation of alveolar (Figure 6B) or bronchiolar outcomes (Figure 6A) especially with MW \#7 closely associated with MW \#2, \#3, and control cells. The additional of the physicochemical characteristics (Supplemental Figure 10-11) with outcomes offered something closer to the in vivo outcomes in that MW \#7 and CNF \#1 grouped as did MW \#1 and control cells. While this was the case, collagen and TGF $\beta$ production group separately. Collagen and SMA group with larger lengths and diameters while TGF $\beta$ grouped with smaller length and diameters (Supplemental Figure 10 and 11B). Previous in vitro studies have investigated the role of TGF $\beta$ in collagen production and found that SWCNT and MWCNT induced TGF $\beta 1$ production and subsequent collagen-1 production through the SMAD signaling cascade [186]. Furthermore, TGF $\beta$ and SMA are associated with the epithelial-mesenchymal transition (EMT) of pulmonary epithelial cells to a migratory cell phenotype associated with cancer and metastasis, and has been found to drive similar EMT outcomes in other nanoparticle exposures including cerium oxide [187189].

While the in vitro data did not fully represent findings in vivo, we can conclude that fibroblast monocultures alone may not accurately represent in vivo findings due to the complex interactions of several cell types and the structure of the extracellular matrix. Alternative, more advanced methods such as co-culture and air-liquid interface models may be more inclusive of the complexities absent in monoculture and may be employed in future experiments. Had a smaller in vitro study been conducted, such as MW \#1 compared to MW\#7 or CNF\#1, the conclusion could have been made that larger physical diameter and length materials cause a greater response when considering outcomes alone. When examining a broader set of materials at one time, those conclusions were not as apparent.

\subsubsection{CNT/F Singlets one day post-exposure}

To assess the particle distribution at $1 \mathrm{~d}$ post-exposure, dark-field microscopy was employed to visualize CNT/F present in the lung tissue. Dark field microscopy allows for the detection and imaging of 
singlets that are not typically visible using standard light microscopy. Representative images are shown in Figure 11. Materials of smaller diameter that form spherical agglomerates are likely to be seen within macrophages (Figure 11A, 11C). However, as the materials increase in diameter becoming less agglomerated, singlets were more abundant (Figure 11D-I). Bundled agglomerates are still frequently seen within macrophages, but some singlets can be seen within the alveolar interstitium. MW \#2 due to its size limited alveolar deposition was mostly found in the bronchioles and the terminal bronchioles (Figure 11B). Singlet lung burden was quantitated and expressed as $100 \%$ of MW \#5, the reference material evaluated previously for translocation [64]. Materials of primarily small nominal tube diameter and length were less likely to be present in singlets. However, with an increase in the number of tubes with larger diameter, the number of singlets increased. MW \#4 and 5 have the greatest number of singlets present in the lung, with MW \#6, \#7, and CNF \#1 having a significant number of singlets present, but less that MW \#4 or \#5 (Figure 12A). CNF \#2 had a moderate burden of singlets, but less than MW \#6, 7, and CNF \#1. It was intriguing that further increasing diameter (e.g., CNF \#1 and \#2) and length (e.g., MW \#7) did not continue to increase singlets within the lung with translocation to the liver suggesting a sweet spot in terms of physical dimensions for MW \#4 and MW \#5.

\subsubsection{Translocation}

$\mathrm{CNT/F}$ mostly are biopersistent materials with minimal alveolar clearance, even up to one year post-exposure [9]. However, while this clearance is minimal, particle is known to translocate from the lung to other systemic tissues including kidney, liver, spleen, brain, and bone marrow [59, 60, 64, 66]. Understanding systemic translocation of CNT/F is important as the ability to induce systemic effects may potentially occur as a direct result of CNT/F interaction on other tissues. Particle which reach the alveolar space will often first be cleared in rodents to the tracheobronchial lymph nodes (TBLN) located in close proximity to the lungs [60]. Green counterstain (Figure 12) was used to visualize black particle 
agglomerates, while dark-field microscopy (Figure 13) was used to assist in the visualization of singlets within the TBLN at $84 \mathrm{~d}$ post-exposure. All materials were able to translocate to the TBLN to various degrees of severity and were often contained within cells. MW \#1 and \#3 were present mostly as spherical agglomerates similar to alveolar deposition at d 1 post-exposure (Figure 11, 13, and 14). However, MW \#4-7, and CNF \#1-2 were found as both bundled agglomerates and singlets (Figure 13D-I; Figure 14D-I). MW \#2, given the large agglomerates/aggregates and greater deposition in the conducting airways, did not translocate to the TBLN with the exception of rarely seen small agglomerates (Figure 13B; Figure 14B).

Of note, many of the singlets present in the TBLN were quite long in length, beyond the ability to be engulfed by a single macrophage. These longer CNT/F may appear in the TBLN as bundles. It is suspected that the particles may arrive as singlets, though their length results in particles being trapped at areas of narrow lymphatic flow, resulting in a dam of particles being generated leading to impaired clearance or potentially damaged lymphatics [64]. Alternatively, macrophages may play a role in particle translocation to the TBLN. CNT/F that are smaller and more tightly agglomerated may be more effectively phagocytosed by macrophages. Therefore, macrophage mediated transport to the TBLN may be greater for materials of smaller agglomerate size (e.g., MW \#1 and \#3).

In conjunction with CNT/F exiting the lung and accumulating in the TBLN, translocation to the liver at d 84 post-exposure was quantified (Figure 12B). This quantification was presented in comparison to MW \#5, the reference material. MW \#1-\#3 had minimal to no particle translocation to the liver. However, MW \#4 had translocation to the liver at a slightly greater level than MW \#5. A representative dark-field micrograph of MW \#4 can be found in Figure 12C. Moderate translocation occurred for MW \#6, \#7, and CNF \#1 ( 25-50\% of MW \#5) with some translocation for CNF \#2 ( 10\% of MW \#5). CNT/F in the liver was only present as singlets. 
Due to the apparent trend between particle singlet burden at 24 hours post-exposure and extra pulmonary translocation of particle to the liver at $84 \mathrm{~d}$ post-exposure, this correlation was assessed using linear regression (Figure 9D). A clear trend can be seen in which particles can be binned into three distinct groups. The first group containing MW \#1-3 has no to minimal translocation to the liver and singlets in the lung at $1 \mathrm{~d}$ post-exposure. The median group has a moderate amount of singlet lung burden with a fractional percentage translocating to the liver. This group contains MW \# 6-7, and CNF \#1-2. The final group contains MW \#4-5. These two materials had the most singlet burden in the lung as well as the highest percentage of liver translocation. Using this trend, it may become possible to predict extra pulmonary translocation to the liver at later time points based solely on particle singlet lung burden at acute time points.

\subsubsection{Hierarchical clustering and PCA of the translocation outcomes}

Clustering of lymph node accumulation, one $\mathrm{d}$ singlet lung burden, and $84 \mathrm{~d}$ accumulation in the liver provided a similar segregation of materials as Figure 14D (Figure 15A). MW \#4 and \#5 were paired and associations between MW \#6, MW \#7, CNF \#1, and CNF \#2 were also present. MW \#2 was very similar to the controls and MW \#1 and \#3 were mostly driven by lymph node accumulation. In the three scenarios of physicochemical characteristics, all variables (Supplemental Figure 12), means only (Supplemental Figure 13), and binned physical dimensions only (Figure 15B), the characteristics illustrating the properties the CNT/F have in dispersion solution, spherical bundles (e.g., MW \#1 and \#3) vs elongated bundled agglomerates (MW \#4-\#7, CNF \#1-\#2) offer a prediction of whether the potential exists for singlets to be found within the lung with subsequent liver accumulation. What was not readily apparent from our analyses was the physicochemical characteristic separating MW \#4 and MW \#5 from the rest of the materials in terms of a greater propensity for liver accumulation. The upper range of length and width dimensions were not a predictive determinant, especially when considering MW \#4 (Figure 
15B). The binning of nominal tube physical dimensions (Figure 15B), as opposed to using means only (Supplemental Figure 13), created a separation of lymph node accumulation from $1 \mathrm{~d}$ pulmonary singlets and $84 \mathrm{~d}$ liver accumulation suggesting that lymph node accumulation alone was not a prerequisite for liver accumulation. The binning also indicated that the smaller length and width parameters (e.g., L2, L4, and W0.15), something that all materials exhibited to some extent, were predictive of lymph node accumulation. These parameters would be consistent with alveolar macrophage uptake and transport to the lymph nodes.

MW \#4 and \#5 accumulating more in the liver compared to other materials suggests multiple contributing factors and/or physicochemical characteristics not determined in this study and may correspond to the rate at which singlets in the lung develop. For example, Mercer et al. 2013 using the same materials as MW \#5 from this study, classified singlets, and groups of 2, 3, 4, or >5 CNT at 1, 14, and $168 \mathrm{~d}$ post-exposure [10]. While MWCNT in agglomerates containing many fibers decreased at 168 d post-exposure, singlet burden did not significantly change. This was concluded to be due to clearance of singlets from the lung, while particle bundles were disassociated into singlets at a rate that gave the impression singlets were not cleared from the lung. While the rate of singlet burden and agglomerate disassociation over time was not determined in this study, it likely would offer insight into the prevalence of MW \#4 and \#5 to accumulate in the liver more readily. The liver accumulation could be the result of cell mediated transport, cell independent transport, or perhaps a combination of both. Previous studies have shown that CNT/F exposure disrupts lung epithelial barriers [190] as well as vascular integrity [88, 191]. This disruption in combination with the lipophilic nature of CNT/F may increase particle passage through membranes and vascular walls resulting in particle entry into systemic blood flow. The combination of the propensity to generate singlets in the lung, the potential for cell dependent and independent transport, suggest a potential nonspecific particle accumulation in tissues such as the liver. 
Interestingly, using the binned approach of physical dimensions, TBLN accumulation was separated from translocation to the liver suggesting a potential different or additional mechanism such as cell mediated transport (Figure 15B). Given MW \#1 and \#3 accumulated in the TBLN like other CNT/F but was rarely visualized in the liver, does not necessarily reject the notion of systemic accumulation in other tissues. MW \#1 and \#3 were observed within cells in the TBLN. If those cells were to enter the general circulation, perhaps immune filtering organs such as the spleen would preferentially have accumulation. While the spleen was not examined for this study, our previous work examined MW \#3 at the same dose and 84 post-exposure time point [19]. The tissues from that study indicated significant accumulation in the spleen as compared to the liver (Supplemental Figure 14). These results suggest differential accumulation systemically depending on whether the material forms singlets in the lung or remains in bundled agglomerates that macrophages can scavenge.

\subsubsection{Summary}

The overall goal of this study was to assess severity in pulmonary histopathology and extrapulmonary particle translocation at a sub-chronic time point from CNT/F exposure of various physicochemical characteristics. In depth histopathological analysis was completed to assess both the severity of tissue changes and injury as well as the regional distribution of those changes. Furthermore, translocation to the proximal lymph nodes and liver were assessed. The standard toxicological outputs indicated obvious differences between materials that was put into perspective using computational analyses.

Previously with a combination of genotoxic responses (Fraser et 2020), CNT/F with larger physical dimensions, a combination of length and diameter, conferred greater toxicity. This was generally the case for histopathology outcomes, especially MW \#5-7 and CNF \#1. Regional specificity was noted for some as MW \#2, a highly entangled material, only had bronchiolar effects while CNT \#2 lacked marked bronchiolar effects. The importance of combined length and width was the separation between 
CNT \#2 and MW \#5-7 and CNF \#1, as CNT \#2 had a reduced length and bundled agglomerate mean length. MW \#1 and \#3, while not observed as singlets in the lung, were not without effect. Those materials, primarily in the L2, L4, and W 0.05 bins exhibited granulomatous inflammation as the small spherical bundles can easily deposit in the deep airways. The lack of larger physical diameter nominal tubes and singlets in MW \#1 and \#3 was the likely reason for a lack of bronchiolar effects and significant alveolar fibrosis.

Prior studies with MW \#5 (Mitsui-7/MWCNT-7) indicated that singlet carbon nanotubes in the lungs were the form responsible for transport and general accumulation in extrapulmonary organs. In the present study, where a wide range of CNT/F species were examined, the one day singlet in the lung was compared to the 84 day liver singlet burden to determine if that relationship between initial lung singlet burden and extrapulmonary transport was present. We found the initial singlet lung burden to be quite variable with very few if any singlets for MW \#1-3. MW \#4-7 and CNF \#1-2 were able to form singlets. Liver accumulation was most apparent for MW \#4 and \#5 followed by MW \#6-7 and CNF \#1.

Evaluating the general similarity in initial lung singlet and liver accumulation indicated the initial dispersion in the lung was a relatively strong determinant for subsequent liver accumulation 84 days later. The significance of those findings suggests the potential for predicting singlet translocation out of the lung early and using computational modeling to group materials can predict which materials were more likely to translocate from the lung.

\subsection{Conclusions}

- The broad class of CNT/F resulted in variable pathology and severity of outcomes. The smaller diameter and length materials, which was present in most all CNT/F, consistently resulted in granulomatous inflammation and alveolar effects. The addition of populations 
of $\mathrm{CNT} / \mathrm{F}$ with both and increased nominal tube diameter and length induced bronchiolar pathology and increased the severity of alveolar pathology.

- $\mathrm{CNT} / \mathrm{F}$ containing nominal tubes of increased length and diameter caused ectasia of the lymphatics. Accumulation at the bronchoalveolar junction may contribute to alveolar fibrosis and pleural accumulation by obstructing clearance mechanisms.

- Similar to our previous evaluations, binning of physical dimensions (length and diameter/width) offered greater resolution in determination of specific physicochemical characteristics contributing to various aspect of developing pathology [10]. The combination of how the CNT/F agglomerate, or two-dimensional sizing, and binning of nominal tube physical dimensions were the best segregators of predicting pathology. Traditional characterization of means only did not offer great resolution for effect determination.

- In terms of toxicity outcome alone and our study design, fibroblasts did not group materials as the in vivo evaluations. Perhaps a different approach than a single cell submerged cultures would provide different outcomes.

- The transition of materials from spherical (MW \#1 and \#3) to elongated bundled agglomerates (MW \#4-7, CNF \#1-2) corresponded to visualization of singlets in the lung.

- Lymph node accumulation was consistent for all CNT/F except for the highly entangled MW \#2. Conversely, liver accumulation was only singlets from MW \#4-7 and CNF \#1-2. In complement, computational modeling using binning of physical dimensions separated lymph node accumulation from day 1 lung singlets and day 84 liver accumulation. Despite the detailed characterization, there was no clear explanation as to why there was a greater propensity for MW \#4 and \#5 to translocate to the liver more proficiently than all other 
materials. Irrespective, day 1 singlets visualized in the lung was a good predictor for accumulation in the liver 84 days later.

- As previously indicated for genotoxicity outcomes, the increased length and diameter $\mathrm{CNT} / \mathrm{F}$ contributing to greater severity in pathological outcomes do not need to be the major fraction of nominal tubes in the sample [10]. A small proportion of tubes with those characteristics were sufficient to alter the severity of the toxicity profile.

\subsection{Materials and Methods:}

\subsubsection{Materials}

Nine materials were assessed in this study including seven multi-walled carbon nanotubes and two carbon nanofibers and are the same materials used in this series of studies. These materials are produced or used in U. S. facilities with the exception of MWCNT \#5, the well-known MWCNT Mitsui-7 which was used as a benchmark material in this study. These materials were extensively characterized as previously reported.

\subsubsection{In vivo study design}

Male C57BL/6J mice ages eight to ten weeks were exposed by oropharyngeal aspiration to either vehicle (physiologic dosing medium; DM), one of seven multi-walled carbon nanotubes (MW \#1, MW \#2, MW \#3, MW \#4, MW \#5, MW \#6, or MW \#7), or one of two carbon nanofibers (CNF \#1 or CNF \#2). Only the high dose of $40 \mu \mathrm{g}$ was used in this study. Previous studies have shown that a $40 \mu \mathrm{g}$ dose of the benchmark material was necessary to induce pathological changes, making it necessary for this study. Mice were euthanized at either 1 or $84 \mathrm{~d}$ post-exposure and tissue was collected to assess changes in histopathology as well as systemic translocation. 


\subsubsection{Animals}

Male C57BL/6J pathogen-free mice weighing 20-25g were obtained from Jackson Laboratories (Bar Harbor, ME) and were housed in the Association for Assessment and Accreditation of Laboratory Animal Care (AAALAC)-accredited NIOSH animal facility. Mice were provided food and tap water ad libitum in ventilated cages, on autoclaved hardwood chip bedding and an environment of controlled humidity, temperature, and 12:12 light/dark cycles. Animals were allowed to acclimate for at least seven $\mathrm{d}$ prior to use in any experiments. Animal care and use procedures were conducted in accordance with the "PHS Policy on Humane Care and Use of Laboratory Animals" and the "Guide for the Care and Use of Laboratory Animals" (2011) and the procedures utilized in this study were approved by the National Institute for Occupational Safety and Health Institutional Animal Care and Use Committee.

\subsubsection{Facility representative material preparation and in vivo dosing}

Fresh dosing media (DM) was prepared prior to dosing. Dosing media contained mouse serum albumin $(0.6 \mathrm{mg} / \mathrm{ml})$ and 1,2-dipalmitoyl-sn-glycero-3-phosphocholine (DPPC; $0.01 \mathrm{mg} / \mathrm{ml})$ prepared in United States Pharmacopeia (USP) grade-phosphate buffered saline (PBS) without calcium and magnesium. To disperse the particles in DM, samples were sonicated for $5 \mathrm{~min}$ at the highest setting using an external sonicator (Hielscher Ultrasound Technology) and then for 5 min using a Branson Sonifier 450 probe sonicator set to the lowest setting (10\% duty cycle; output control of 1). Mice were dosed by oropharyngeal aspiration according to the well-established protocols previously described [179].

\subsubsection{Tissue collection}

Mice were sacrificed at either 1 or $84 \mathrm{~d}$ post-exposure. Following an intraperitoneal injection of fatal plus, the abdomen was exposed, and mice were exsanguinated. The right lung was ligated and inflated with $10 \%$ buffered formalin by gravity fixation. The liver and tracheobronchial lymph node were also 
removed and formalin fixed. Fixed tissue was then embedded in paraffin wax, cut into $5 \mu \mathrm{m}$ sections, and mounted on slides for staining.

\subsubsection{General Histopathology}

$\mathrm{H} \& \mathrm{E}$ and trichrome stained tissue section were assessed by a board-certified veterinary pathologist. At $84 \mathrm{~d}$ post-exposure to $40 \mu \mathrm{g}$ CNT/F or vehicle ( $\mathrm{n}=4-8$ per group), The right lung was fixed in $10 \%$ neutral-buffered formalin and then stained with either Masson's trichrome stain or hematoxylin and eosin (H\&E). Two semi-quantitative scores were assigned for each morphologic alteration. The severity score is the numerical equivalent of the following intensities of tissue morphologic change: $0=$ none, $1=$ minimal, $2=$ mild, $3=$ moderate $4=$ marked, and $5=$ severe. The second was a distribution score to quantify the extent (amount) of the tissue involvement. The distribution scoring is as follows: $0=$ none, $1=$ focal, $2=$ locally extensive, $3=$ multifocal, $4=$ multifocal and coalescent, and $5=$ diffuse. These scores were combined to generate a total score ranging from 0-10 with 10 a severe and diffuse injury. These scores as well as incidences are listed in Table 2 and representative micrographs of the morphologic alterations are found in Figure 2.

Lung tissue from $84 \mathrm{~d}$ post-exposure was sectioned and mounted on slides where it was stained with either Masson's Trichrome stain or hematoxylin and eosin (H\&E). H\&E-stained slides were examined by a veterinary pathologist using bright field microscopy and polarizing light microscopy. Changes in morphology as well as areas of tissue injury and inflammation were assessed and scored for both severity $(1=$ minimal, $2=$ mild, $3=$ moderate, $4=$ moderately severe , and $5=$ severe $)$ and distribution ( 1 =focal, $2=$ locally extensive, $3=$ multifocal, $4=$ multifocal and coalescent, $5=$ diffuse $)$. These scores were then added together to create a total score out of a possible 10, with 10 being the most severe and diffuse. Photomicrographs were captured using an Olympus BX53 microscope equipped with a DP73 camera (Olympus, Tokyo, Japan). 


\subsubsection{Morphometry and alveolar fibrosis}

Morphometric analysis was used to measure changes in alveolar fibrosis. Lung sections from $84 \mathrm{~d}$ post-exposure were stained with picrosirius red to detect collagen fibers as previously described [10]. Briefly, $5 \mu \mathrm{m}$ sections of lung were immersed in $0.1 \%$ Picrosirius solution for $1-2$ hours and were rinsed with $0.01 \mathrm{~N}$ HCL. Subsequent counterstaining with hematoxylin was completed followed by coverslipping. Quantitative morphometric analysis was used to determine the volume density of alveolar collagen and measure alveolar collagen thickness using basic point and intercept counting. Point and intercept counts were made using an 11-line overlay graticule (12.5 mm square with 100 divisions), at 100x magnification, taken at eight locations equally spaced across each section. One section was used per animal with a total of 4-7 animals per treatment group. (Weibel ER. Stereological Methods: Practical Methods for Biological Morphometry. 1. New York, NY: Academic Press; 1980.)

\subsubsection{Bronchiolar fibrosis}

To assess the severity of bronchiolar fibrosis at $84 \mathrm{~d}$ post-exposure, images of the broncho-alveolar duct in the most affected regions were taken using an Olympus BX63 microscope equipped with a DP73 camera and CellSens Dimension software (Olympus Corporation, Tokyo, Japan). For each animal lung section, a total of six images were taken. Three images were taken of most affected junctions with minimal severity of bronchiolar fibrosis. Additionally, three images were taken of most affected junctions representing the most severe fibrosis. Only three images were taken of control animals. For each treatment, one section per mouse was taken for a total of $n=4-7$ per treatment group. Bronchiolar fibrosis was quantified using ImageJ (National Institutes of Health, Bethesda, MD) as previously described [192]. Briefly, using the color deconvolution pluggin, the image was separated into color channels, with green representing areas of fibrosis. The areas of bronchiolar fibrosis were selected, and the area and percent area was quantified. This percent area was then divided by the total area of the field of view to quantify 
the total percent area of fibrosis. Additionally, the area was then normalized to the length of the basement membrane to express the fibrosis as a measurement of area per micron of basement membrane.

\subsubsection{Lung and liver particle burden}

The singlet lung burden and total liver particle burden was quantified using dark field microscopy to visualize particle present in lung and liver tissue as previously described [10]. Morphometric point and intercept counting was used as earlier described in the quantification of alveolar fibrosis, however only lung singlets and all liver particles were counted and quantified. Particle in the liver was only present as singlets.

\subsubsection{Translocation}

Tracheobronchial lymph nodes (TBLN) were sectioned and mounted onto plus slides as previously described and were stained with green counterstain to visualize the presence of black particle. Furthermore, additional sections of the lung, TBLN, and liver were mounted onto Schott slides. Using CytoViva, (CytoViva; Auburn, AL) images of the lymph nodes were collected to visualize particle deposition and extra-pulmonary accumulation in the liver and lymph nodes.

\subsubsection{In vitro Study Design}

Primary normal human lung fibroblasts were exposed to either DM or one of one of the nine $\mathrm{CNT} / \mathrm{F}$ at doses relevant to human occupational exposure to assess cytotoxicity and the potential mechanisms by which histopathological changes may be occurring. Additionally, this study aims to find correlations between in vivo and in vitro experimental findings to determine the degree to which in vitro methods may predict in vivo toxicity. 


\subsubsection{Cell culture}

Primary normal human lung fibroblasts (NHLFs) were acquired from Lonza (Walkersville, MD) and cultured at sub-confluent densities in fibroblast growth medium which contained FGM2 BulletKit growth supplements (FGM2, Lonza) and $100 \mathrm{U} / \mathrm{ml}$ penicillin/streptomycin as previously described [193]. Briefly, cells were passaged by washing with a HEPES-based buffer, suspension via 5\% trypsin, and neutralization using trypsin neutralization buffer (Lonza) following manufacturer's procedures. Cells maintained in a 37 ${ }^{\circ} \mathrm{C}$ incubator in a humid, $5 \% \mathrm{CO}_{2}$ atmosphere. $3^{\text {rd }}$ to $7^{\text {th }}$ passage NHLFs were used for all assays.

\subsubsection{MWCNT preparation and dispersion}

Dry MWCNT were weighed and placed into labeled, separate Eppendorf tubes. Dispersion medium (DM) containing $0.6 \mathrm{mg} / \mathrm{ml}$ albumin and $10 \mu \mathrm{g} / \mathrm{ml}$ DPPC in sterile phosphate-buffered saline (PBS) was freshly made for each assay. For each assay, DM was added to each dry MWCNT sample to achieve a $3 \mathrm{mg} / \mathrm{ml}$ stock solution. Next, MWCNTs were sonicated for 5 minutes using an external sonicator (Hielscher Ultrasound Technology) at $4{ }^{\circ} \mathrm{C}$ as an initial dispersion procedure. Then, each MWCNT was then hand sonicated for 20 seconds using a microtip sonicator (Fisher Scientific) at $4{ }^{\circ} \mathrm{C}$ three separate times and immediately serially diluted in FGM2 medium for cellular assays.

\subsubsection{WST-1 assay}

To screen MWCNT cytotoxicity and changes in cell metabolism, WST-1 assay was conducted as previously described with modifications [194]. Briefly, NHLFs were passaged and seeded into tissue culture-treated 96-well plates (Corning Inc., Corning, NY) at 5,000 cells per well overnight in $100 \mu \mathrm{l}$ volume. Cells were then exposed to seven dilutions of each MWCNT in fresh medium ranging $0.02-20$ $\mu \mathrm{g} / \mathrm{cm}^{2}$ in $200 \mu \mathrm{l}$ volume along with unexposed cells and medium-only blanks for 24 hours. All treatment groups were run in triplicate. 1 hour prior to the assay time point, $1 \%$ Triton-X solution was added to a 
subset of unexposed cells to serve as a $100 \%$ cytotoxicity control. Next, WST-1 reagent was added to each well and the plate was allowed to incubate for 2 hours in the culture incubator following manufacturer's instructions (Roche LifeScience, Indianapolis, IN). Absorbance of metabolized WST-1 was read on a SpectraMax 250 microplate spectrophotometer (Molecular Devices, Sunnyvale, CA) at 462 nm while 650 nm served as a background measure. Absorbance values were subtracted from mean blank values and means calculated. Three independent assays were run. Since high MWCNT concentrations in the wells increased baseline absorbance values, two separate assays with the same MWCNT dose scheme, but without cells, served as particle controls and were used to correct absorbance values for all cellular assays. Mean absorbance values were converted to percent viability.

\subsubsection{Collagen I, $\alpha$-SMA, and TGF $\beta$ expression}

MWCNTs were screened for pro-fibrotic markers in exposed NHLFs as evidence for direct fibroblast stimulation ability as previously described [186, 195]. Briefly, suspended NHLFs were seeded into tissue culture-treated 6-well plates (Corning) at 3E5 cells per well overnight. NHLFs were then exposed to 0.02, 0.06 , and $0.2 \mu \mathrm{g} / \mathrm{cm}^{2}$ in $2 \mathrm{ml}$ volume for 48 hours, which match a $10 \mu \mathrm{g}, 30 \mu \mathrm{g}$, and $100 \mu \mathrm{g}$ dose per mouse lung assuming $500 \mathrm{~cm}^{2}$ surface area [195]. Unexposed cells and $1 \mathrm{ng} / \mathrm{ml}$ human TGF $\beta$ served as negative and positive controls. Since the design was spread across four plates, one well of unexposed cells on each plate served as a plate control. Following exposure, digital phase contrast images of dispersed MWCNTs on NHLFs were acquired at 10X and 20X using a Revolve microscope (ECHO, San Diego, CA). Cell lysates were prepared for western blot analysis. Briefly, after plates were chilled on ice for 5 minutes, conditioned medium was collected into Eppendorf tubes, centrifuged at 1000 rpm for 5 minutes to pellet cell debris, followed by the collection and storage of the supernatant at $-80{ }^{\circ} \mathrm{C}$ for TGF $\beta$ assay. Collected conditioned medium was assessed in technical triplicate for secreted total TGF $\beta$ concentrations via DuoSet ELISA after latent TGF $\beta$ activation via acid incubation following manufacturer's instructions 
(RandD Biosystems, Minneapolis, MN) as previously described[186]. Absorbance was measured at 450 nm on a 96-well microplate reader.

Next, exposed NHLFs were washed in cold PBS followed incubation in lysis buffer (Invitrogen, Carlsbad, CA) containing 0.1 mM PMSF and complete protease inhibitor cocktail. Lysate samples were scraped, collected into tubes, briefly homogenized using microtip sonication, and centrifuged at 12,500 rpms for 15 min at $4{ }^{\circ} \mathrm{C}$. Collected supernatants were assayed for total protein using a BCA kit following manufacturer instructions (Pierce, Rockford, IL). $30 \mu \mathrm{g}$ protein samples were separated on 7\% SDSPAGE gels followed by semi-dry transfer (Fisher Scientific, Hampton, NH) to nitrocellulose membranes. Following blocking in $0.5 \%$ or $5 \%$ non-fat dry milk in TBS buffer with $1 \%$ Tween20 (TBS-T), membranes were probed for rabbit Collagen I (Fitzgerald, Acton, MA), rabbit $\alpha$-SMA (AbCam, Cambridge, MA), and mouse monoclonal $\beta$-actin (Sigma Aldrich) using primary antibodies either using MiniBlot 2.0 system at room temperature (Millipore, Burlington, MA) or overnight at $4{ }^{\circ} \mathrm{C}$. After rinsing thrice with TBS-T, membranes were incubated with either rabbit or mouse HRP-conjugated secondary antibodies (Santa Cruz Biotechnology, Dallas, TX) for 1 hour at room temperature. Lastly, membranes were incubated with SuperSignal chemiluminescent substrate (ThermoFisher Scientific, Waltham, MA) for 5 minutes and then exposed to X-ray film. Films with bands were digitized and densitometry performed on ImageJ. Protein expression was calculated as fold change compared to unexposed controls following correction using $\beta$ actin expression for each sample. All experiments were performed three independent times.

\subsubsection{Feature selection and principal component analysis}

To permit selection of the minimal number of features among all characterization and L-W properties that could potentially discriminate between each material investigated, feature selection was performed with a random forest-based approach [169] using the "Boruta" algorithm [170] in the R statistical environment [171]. The Boruta algorithm adds randomness to the variables in the dataset by 
creating shuffled copies of all variables ("shadow features"). "Boruta" iteratively assesses if each variable has a higher Z-score than the maximum Z-score of its shadow features. At each iteration, variables with Z-scores lower than shadow features are deemed unimportant and removed subsequently by the algorithm to capture all the important, interesting features one might have in the dataset with respect to a dependent variable, in this case each material itself. Then, using traditional, L-W-AR, and combined variables retained after applying the "Boruta" algorithm, principal component analysis (PCA) was performed to identify significant patterns that explained the majority of the variations in the physicochemical properties among the different CNT/F materials investigated. PCA was performed using the prcomp command of the R statistical software (R Core Team, 2016).

\subsubsection{Statistics}

Data are presented as mean with standard error or standard deviation as indicated in the figure legends. Figures were prepared using SigmaPlot software (Systat Software, INC). Statistical analysis used include one-way analysis of variance. Differences were considered significant at $\mathrm{p}<0.05$. This analysis was performed using SAS/STAT software, Version 9.4 of the SAS system for Windows (SAS Institute, Cary, NC). 


\section{CHAPTER 4 TABLES}

Table 1: Histopathology scores for distribution, severity, total score, and incidence at 84 days postexposure

\begin{tabular}{|c|c|c|c|c|c|c|c|c|c|c|c|}
\hline & & DM & MW\#1 & MW\#2 & MW \#3 & MW\#4 & MW $\# 5$ & MW\#6 & MW\#7 & CNF \#1 & $\mathrm{CNF} \# 2$ \\
\hline \multirow{4}{*}{ G ranulomatous In flammation } & Disttibution & $0.00 \pm 0.00$ & $3.00 \pm 0.00^{*}$ & $3.00 \pm 0.00^{*}$ & $3.00 \pm 0.00^{*}$ & $3.00 \pm 0.00^{*}$ & $3.20 \pm 0.20^{*}$ & $3.00 \pm 0.00^{*}$ & $3.00 \pm 0.00^{*}$ & $3.00 \pm 0.00^{*}$ & $2.17 \pm 0.54^{*}$ \\
\hline & Severity & $0.00 \pm 0.00$ & $2.50 \pm 0.29^{*}$ & $2.50 \pm 0.22^{*}$ & $2.20 \pm 0.37^{*}$ & $3.00 \pm 0.00^{*}$ & $3.20 \pm 0.20^{*}$ & $3.00 \pm 0.00^{*}$ & $3.17 \pm 0.00^{*}$ & $3.00 \pm 0.00^{*}$ & $1.50 \pm 0.43^{*}$ \\
\hline & Total Score & $0.00 \pm 0.00$ & $5.55 \pm 0.29^{*}$ & $5.50 \pm 0.22^{\star}$ & $5.20 \pm 0.37^{\star}$ & $6.00 \pm 0.00^{*}$ & $6.40 \pm 0.40^{*}$ & $6.00 \pm 0.00^{*}$ & $6.17 \pm 0.00^{*}$ & $6.00 \pm 0.00^{*}$ & $3.67 \pm 0.92^{*}$ \\
\hline & Incidence & a/7 & $4 / 4$ & $6 / 6$ & $5 / 5$ & $6 / 6$ & $5 / 5$ & $6 / 6$ & $6 / 6$ & $5 / 5$ & $5 / 6$ \\
\hline \multirow{4}{*}{$\begin{array}{l}\text { Bronchiolitis Obliterans-like } \\
\text { Changes }\end{array}$} & Distribution & $0.00 \pm 0.00$ & $0.00 \pm 0.00$ & $1.67 \pm 0.61^{*}$ & $0.20 \pm 0.20$ & $1.17 \pm 0.60$ & $0.00 \pm 0.00$ & $2.50 \pm 0.50^{\star}$ & $2.67 \pm 0.33^{*}$ & $1.40 \pm 0.68^{*}$ & $0.00 \pm 0.00$ \\
\hline & Severity & $0.00 \pm 0.00$ & $0.00 \pm 0.00$ & $2.00 \pm 0.63^{*}$ & $0.60 \pm 0.60$ & $1.17 \pm 0.60$ & $0.00 \pm 0.00$ & $2.5 \pm 0.50^{\star}$ & $3.00 \pm 0.26^{*}$ & $1.20 \pm 0.49^{*}$ & $0.00 \pm 0.00$ \\
\hline & Total Score & $0.00 \pm 0.00$ & $0.00 \pm 0.00$ & $3.67 \pm 1.20^{*}$ & $0.80 \pm 0.80$ & $2.33 \pm 1.20$ & $0.00 \pm 0.00$ & $5.00 \pm 01.00^{*}$ & $5.67 \pm 042^{*}$ & $2.60 \pm 1.12^{*}$ & $0.00 \pm 0.00$ \\
\hline & Incidence & $0 / 7$ & $0 / 4$ & $4 / 6$ & $1 / 5$ & $3 / 6$ & $0 / 5$ & $5 / 6$ & $6 / 6$ & $3 / 5$ & $0 / 6$ \\
\hline \multirow{3}{*}{ Alveolar Histiocy to sis } & Severity & $0.00 \pm 0.00$ & $1.50 \pm 0.50^{*}$ & $0.33 \pm 0.21$ & $0.80 \pm 0.37^{\star}$ & $1.00 \pm 0.37^{\star}$ & $3.00 \pm 0.00^{*}$ & $1.33 \pm 0.49^{*}$ & $2.83 \pm 0.48^{*}$ & $2.40 \pm 0.51^{*}$ & 1. $83 \pm 0.48^{*}$ \\
\hline & Total Score & $0.00 \pm 0.00$ & $3.75 \pm 1.25^{\star}$ & $1.00 \pm 0.68$ & $2.60 \pm 1.08^{*}$ & $2.67 \pm 0.95^{\star}$ & $6.00 \pm 0.00^{*}$ & $3.33 \pm 1.09^{*}$ & $5.50 \pm 0.43^{*}$ & $5.40 \pm 0.51^{*}$ & $4.50 \pm 0.99^{*}$ \\
\hline & Incidence & $\alpha / 7$ & $3 / 4$ & $2 / 6$ & $3 / 5$ & $4 / 6$ & $5 / 5$ & $4 / 6$ & $6 / 6$ & $5 / 5$ & $5 / 6$ \\
\hline \multirow{4}{*}{ Bronchiolar Epithelial Hypertrophy } & Disrtibution & $0.00 \pm 0.00$ & $1.50 \pm 0.87$ & $3.00 \pm 0.00^{*}$ & $0.60 \pm 0.60$ & $3.00 \pm 0.00^{*}$ & $3.00 \pm 0.00^{*}$ & $3.00 \pm 0.00^{*}$ & $3.00 \pm 0.31^{*}$ & $3.00 \pm 0.00^{*}$ & $2.50 \pm 0.50^{*}$ \\
\hline & Severity & $0.00 \pm 0.00$ & $1.00 \pm 0.71$ & $2.50 \pm 0.34^{*}$ & $0.40 \pm 0.40$ & $1.83 \pm 0.40^{*}$ & $2.80 \pm 0.20^{*}$ & $3.00 \pm 0.26^{\star}$ & $3.17 \pm 0.31^{\star}$ & $3.40 \pm 0.24^{*}$ & $2.17 \pm 0.54^{*}$ \\
\hline & Total Score & $0.00 \pm 0.00$ & $2.50 \pm 1.50$ & $5.50 \pm 0.34^{*}$ & $1.00 \pm 1.00$ & $4.83 \pm 0.40^{*}$ & $5.80 \pm 0.20^{*}$ & $6.00 \pm 0.26^{*}$ & $6.17 \pm 0.31^{*}$ & $6.40 \pm 0.24^{*}$ & 4. $67 \pm 0.99^{*}$ \\
\hline & Incidence & $0 / 7$ & $2 / 4$ & $6 / 6$ & $1 / 5$ & $6 / 6$ & $5 / 5$ & $6 / 6$ & $6 / 6$ & $5 / 5$ & $5 / 6$ \\
\hline \multirow{3}{*}{$\begin{array}{l}\text { Alveolar Epithelial Hypertrophy and } \\
\text { Hyperplasia }\end{array}$} & Distribution & $0.00 \pm 0.00$ & $2.25 \pm 0.75^{*}$ & $0.17 \pm 0.17$ & $1.60 \pm 0.60^{*}$ & $2.67 \pm 0.33^{*}$ & $3.00 \pm 0.00^{*}$ & $2.50 \pm 0.50^{\star}$ & $2.67 \pm 0.33^{*}$ & $3.00 \pm 0.00^{*}$ & $2.50 \pm 0.50^{*}$ \\
\hline & Severity & $0.00 \pm 0.00$ & $0.75 \pm 0.25^{\star}$ & $0.17 \pm 0.17$ & $1.20 \pm 0.49^{*}$ & $1.50 \pm 0.22^{*}$ & $3.40 \pm 0.24^{*}$ & $1.67 \pm 0.42^{*}$ & $2.83 \pm 0.40^{*}$ & $3.00 \pm 0.45^{*}$ & 1. $67 \pm 0.49^{*}$ \\
\hline & Incidence & $a / 7$ & $3 / 4$ & $1 / 6$ & $4 / 5$ & $6 / 6$ & $5 / 5$ & $5 / 6$ & $6 / 6$ & $5 / 5$ & $5 / 6$ \\
\hline \multirow{4}{*}{ Bronchial or Bronchiolar Fibrosis } & Distribution & $0.00 \pm 0.00$ & $1.75 \pm 0.75^{\star}$ & $3.00 \pm 0.00^{*}$ & $1.40 \pm 0.68^{*}$ & $250 \pm 0.50^{*}$ & $3.00 \pm 0.00^{*}$ & $3.00 \pm 0.00^{*}$ & $2.67 \pm 0.33^{*}$ & $3.00 \pm 0.00^{*}$ & 1. $67 \pm 0.61^{*}$ \\
\hline & Severity & $0.00 \pm 0.00$ & 1. $25 \pm 0.48^{*}$ & $1.20 \pm 0.20^{*}$ & $0.80 \pm 0.37^{*}$ & $1.83 \pm 0.48^{*}$ & $3.80 \pm 0.20^{*}$ & $3.17 \pm 0.31^{*}$ & $3.83 \pm 0.17^{\star}$ & $3.00 \pm 0.32^{*}$ & $0.83 \pm 0.31^{*}$ \\
\hline & Total Score & $0.00 \pm 0.00$ & $3.00 \pm 1.22^{*}$ & $4.20 \pm 0.20^{*}$ & $2.20 \pm 0.92^{*}$ & $4.33 \pm 0.92^{*}$ & $6.80 \pm 0.20^{*}$ & $6.17 \pm 0.31^{*}$ & $6.50 \pm 0.34^{*}$ & $6.00 \pm 0.32^{*}$ & $250 \pm 0.89^{*}$ \\
\hline & Incidence & $Q 17$ & $3 / 4$ & $5 / 5$ & $3 / 5$ & $5 / 6$ & $5 / 5$ & $6 / 6$ & $6 / 6$ & $5 / 5$ & $4 / 6$ \\
\hline \multirow{4}{*}{ Alveolar In terstitial F ibrosis } & Disrtibution & $0.00 \pm 0.00$ & $3.00 \pm 0.00^{*}$ & $2.60 \pm 0.40^{*}$ & $1.80 \pm 0.73^{*}$ & $3.00 \pm 0.00^{*}$ & $3.00 \pm 0.00^{*}$ & $3.00 \pm 0.00^{\star}$ & $3.00 \pm 0.00^{*}$ & $3.00 \pm 0.00^{*}$ & $2.17 \pm 0.54^{*}$ \\
\hline & Severity & $0.00 \pm 0.00$ & $1.25 \pm 0.25^{\star}$ & $1.00 \pm 0.00^{*}$ & $0.60 \pm 0.24^{*}$ & $2.50 \pm 0.34^{\star}$ & $3.80 \pm 0.20^{*}$ & $2.33 \pm 0.21^{*}$ & $2.67 \pm 0.21^{*}$ & $2.40 \pm 0.40^{*}$ & 1. $17 \pm 0.31^{*}$ \\
\hline & Total Score & $0.00 \pm 0.00$ & $4.25 \pm 0.25^{\star}$ & $3.60 \pm 0.40^{*}$ & $2.40 \pm 0.98^{*}$ & $5.50 \pm 0.34^{*}$ & $6.80 \pm 0.20^{*}$ & $5.33 \pm 0.21^{*}$ & $5.67 \pm 0.21^{*}$ & $5.40 \pm 0.40^{*}$ & $3.33 \pm 0.80^{*}$ \\
\hline & Incidence & $\alpha / 7$ & $4 / 4$ & $5 / 5$ & $3 / 5$ & $6 / 6$ & $5 / 5$ & $6 / 6$ & $6 / 6$ & $5 / 5$ & $5 / 6$ \\
\hline
\end{tabular}




\section{CHAPTER 4 FIGURES}
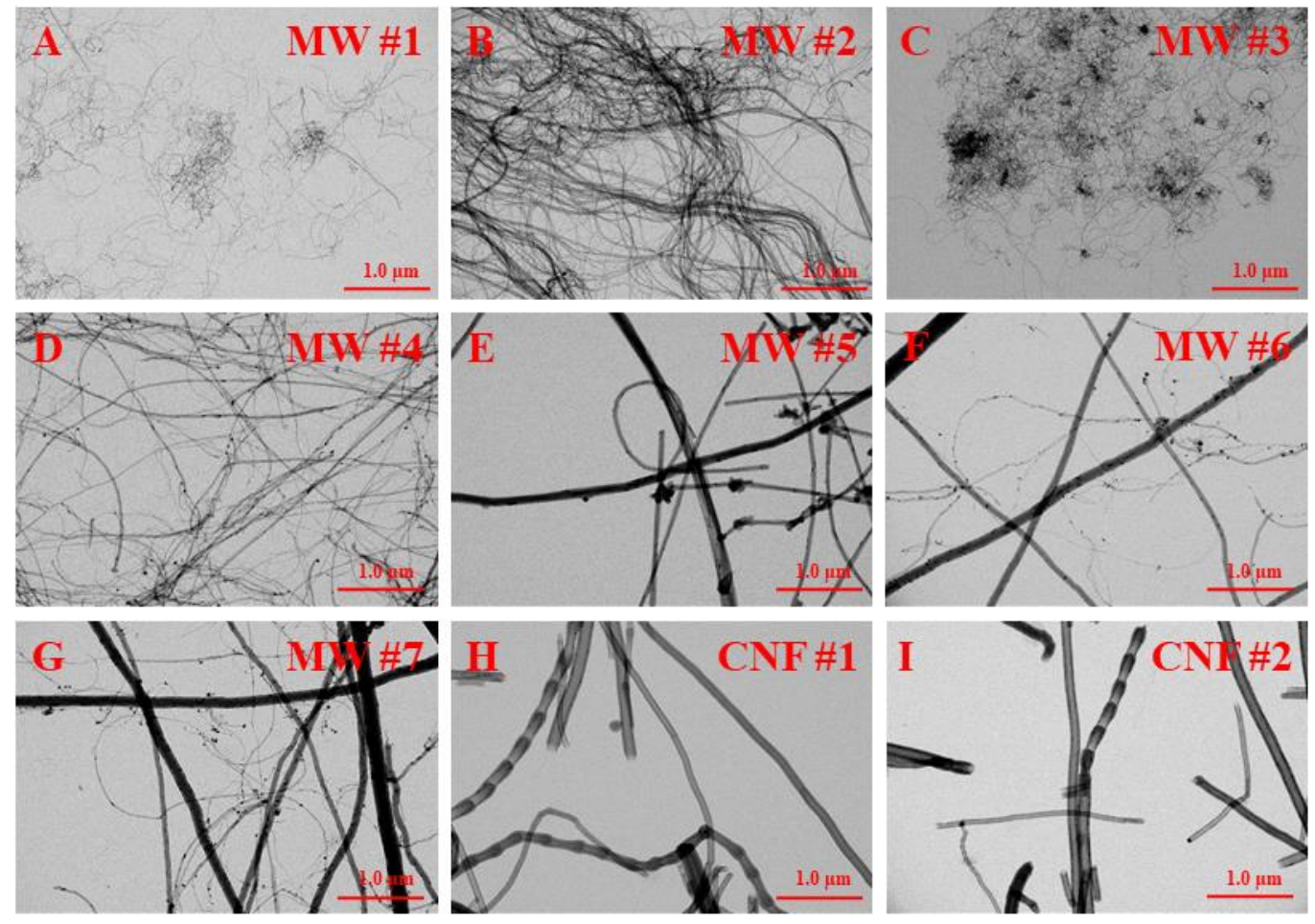

Figure 4.1. Representative TEM images of particle dispersed in isopropanol. Scale bar represents $1 \mu \mathrm{m}$. Images A-I are of MW \#1, 2, 3, 4, 5, 6, 7, and CNF \#1, and 2 respectively. These images serve to illustrate differences in particle size and agglomeration patterns. Materials of small diameter and length (MW \#1, 3) tend to be more heavily agglomerated in tangled spherical agglomerates. MW \#2 is a unique material that forms large and long rivers of agglomerates. As materials increase in diameter and length, the agglomeration becomes looser and singlets are more common. Some materials including MW \#6 and \#7 have mixed populations of sizes with some small, moderate, and large diameter tubes present. 

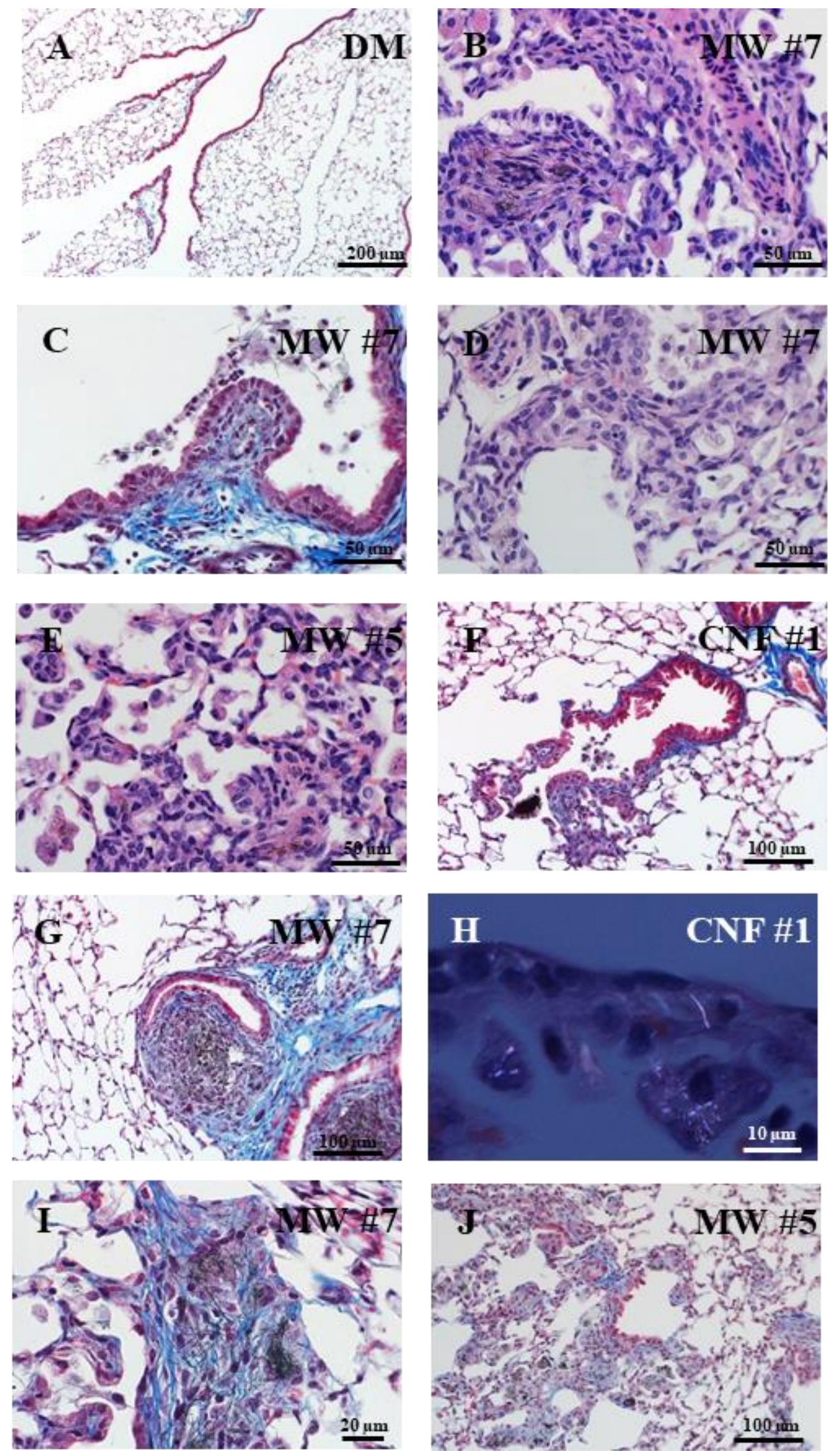
Figure 4.2. Representative micrographs at $84 \mathrm{~d}$ post-exposure of pathologies typically seen following MWCNT and CNF exposure. A) Representative trichrome stained image from the lung of a DM exposed mouse demonstrates healthy tissue and airways. B) Granulomatous Bronchopneumonia is an encapsulation of foreign material by inflammatory cells seen following exposure to all materials. Representative image is of MW \#7 exposed lung tissue stained with H\&E. Particles are frequently visible within these encapsulations. C) Bronchiolitis obliterans-like changes were seen following exposure to MW \#2, 6, 7, and CNF \#1 in areas of high of particle deposition in the bronchioles. Representative image is taken from MW \#7 exposed lung section. D) Alveolar histiocytosis, hypertrophy, and hyperplasia is identifiable by a lesion of inflammatory cell concentration in the peribronchiolar and alveolar regions and as thickening of alveolar epithelium. This is common seen following exposure to most MWCNTs and CNFs in this study except MW \#2. Representative image is of MW \#7 exposed lung following H\&E staining. E) Morphologic alterations in this section of mouse lung from a MW5-exposed mouse include alveolar epithelial cell hypertrophy and hyperplasia, alveolar histiocytosis, and interstitial fibrosis. MW5 nanotubes are retained in this lung section, and because they block transmitted light, are demonstrable in this section as dark fiber-like structures in alveolar macrophages and the fibrotic interstitium. F) Bronchiolar epithelium hypertrophy is also commonly observed as a thickening of the epithelial layer of the bronchioles. Representative image is of CNF \#1 exposed lung. G) Disruption of lymphatic flow can be seen following exposure to several materials including MW \#7 as seen in the representative image. Dark particles can be seen in a massive tangle forming an obstruction and subsequent back flow of the lymphatics. H) Pleural penetrations were seen following exposure to MW \#5, 6, and CNF \#1 and 2 in at least one occurrence. Representative image is a partially polarized image of H\&E stained tissue from CNF \#1 exposed mouse tissue. I) Bronchiolar fibrosis and J) alveolar interstitial fibrosis is a thickening of collagen deposition in the broncho-alveolar duct region and the alveolar interstitium respectively Representative images are of MW \#7 and MW \#5 exposed lungs, respectively, with Masson's trichrome 
stain. All materials induced this response with variable severity and distribution with correlation to the region of particle deposition. 

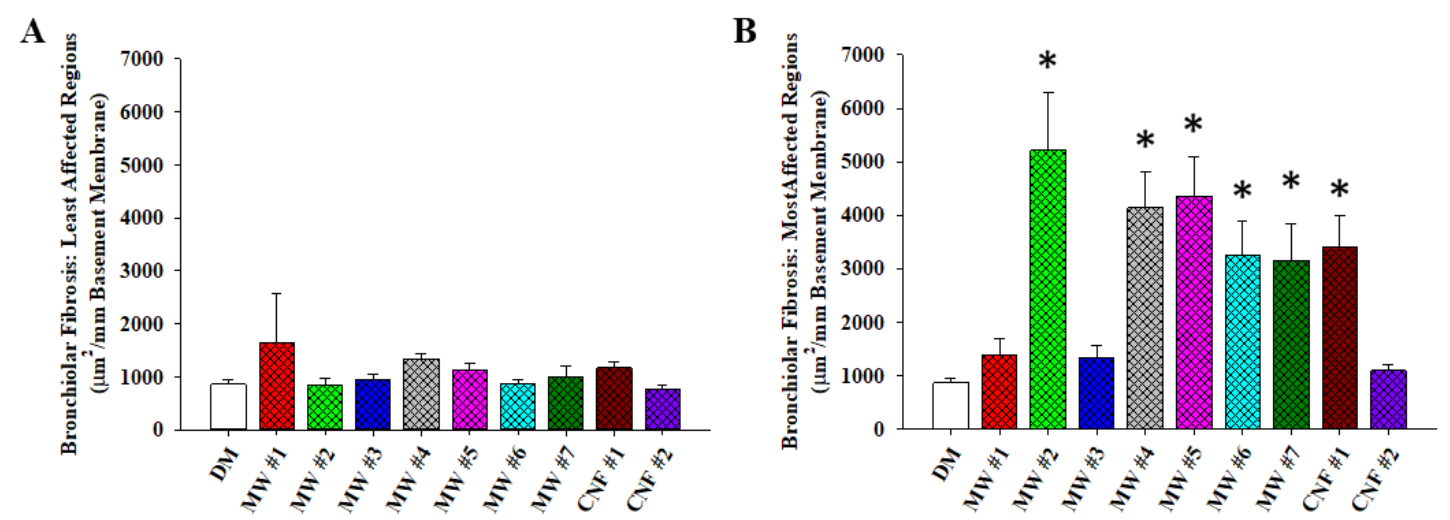

Figure 4.3. Bronchiolar Fibrosis at 84 days post-exposure to $40 \mu \mathrm{g}$ dose of MWCNT or CNF. The total area of bronchial/bronchiolar fibrosis per mm of basement membrane was quantified in two regions, both the least affected and most affected regions for comparison. While all treatment groups had normal, unaffected bronchoalveolar duct regions, MW \#2, 4-7, and CNF \#1 had severely impacted bronchi/bronchioles. $\left({ }^{*} \mathrm{p}<0.05\right)$ 

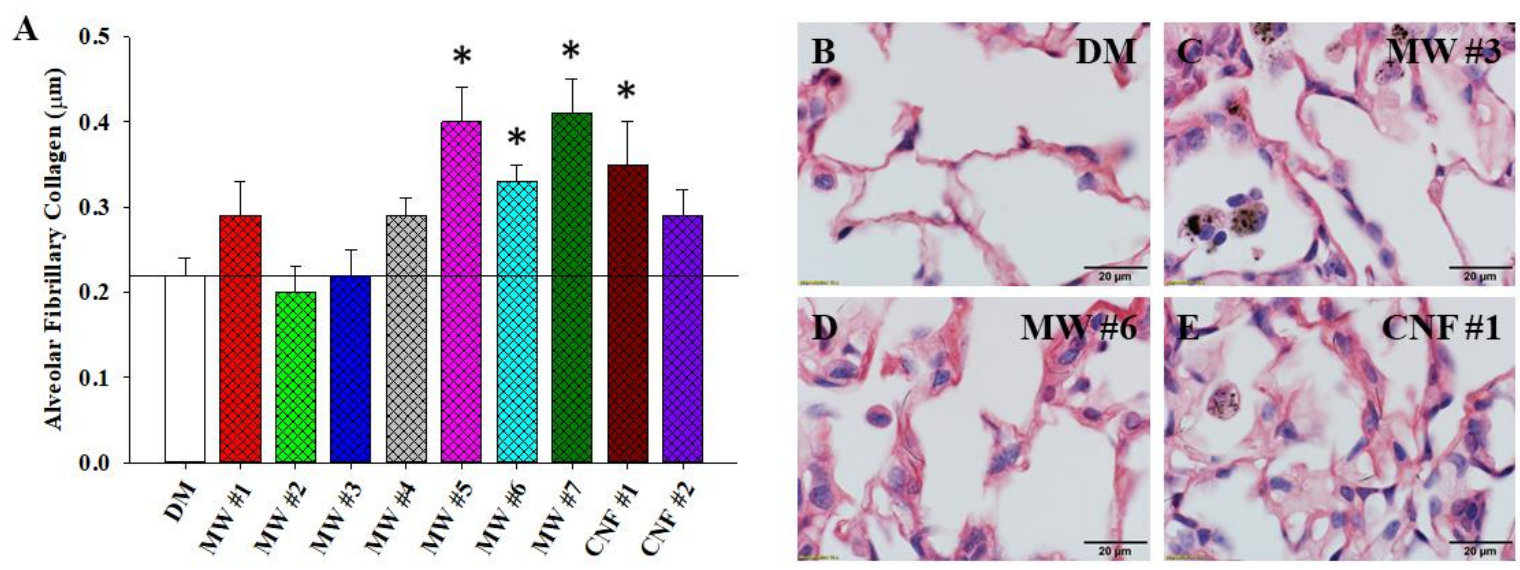

Figure 4.4. Alveolar Fibrillary Collagen quantification. A) At 84 days post-exposure to the high dose of MWCNTs or CNFs, the thickness of alveolar fibrillary collagen was measured using morphometric point and intercept counting. Reference line is relative to DM. Statistically significant increases were seen following exposure to MW \#1, \#4-7, and CNF \#1-2. MW \#2 deposition is limited to the conducting airways due to particle agglomerate size. While MW \#3 does reach the alveolar region, fibrosis is minimal overall. Representative micrographs of picrosirius red stained tissue are as follows: B) DM, C) MW \#3, D) MW \#6, and CNF \#1. Particle is visibly present in the interstitial walls in D and E. (*p<0.05) 

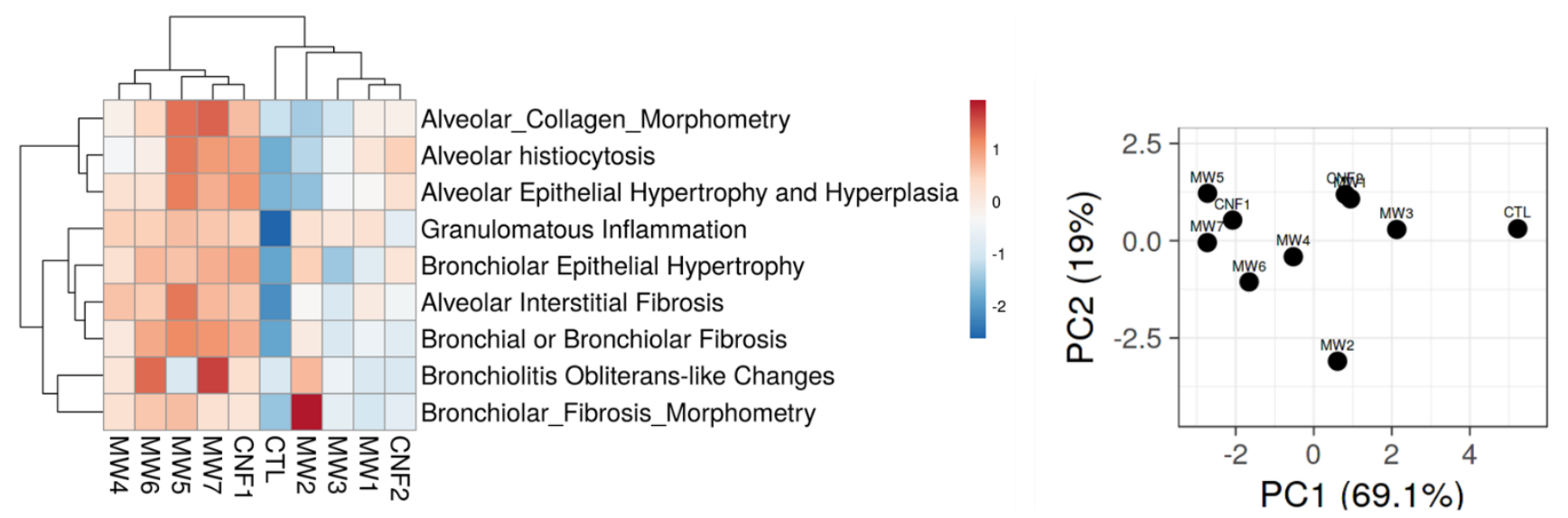

Figure 4.5. Hierarchical clustering analysis (HCA) and principal component analysis (PCA) of different CNT/F materials comparing MWNCT/CNF and histopathology outcomes. 
A

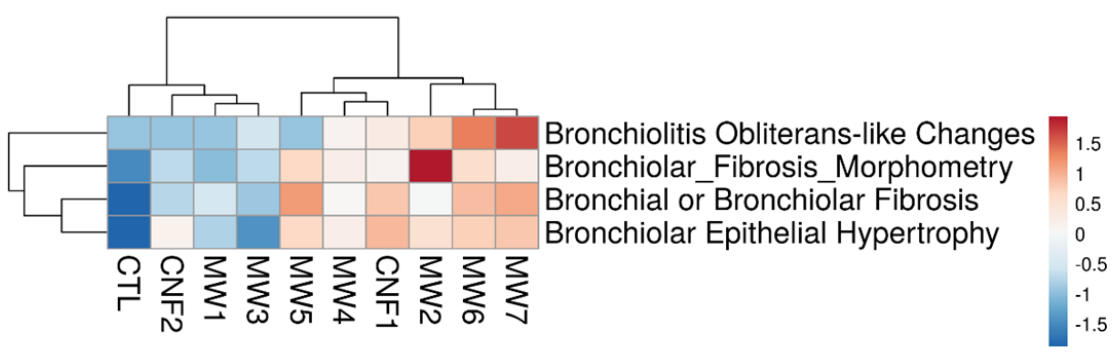

B

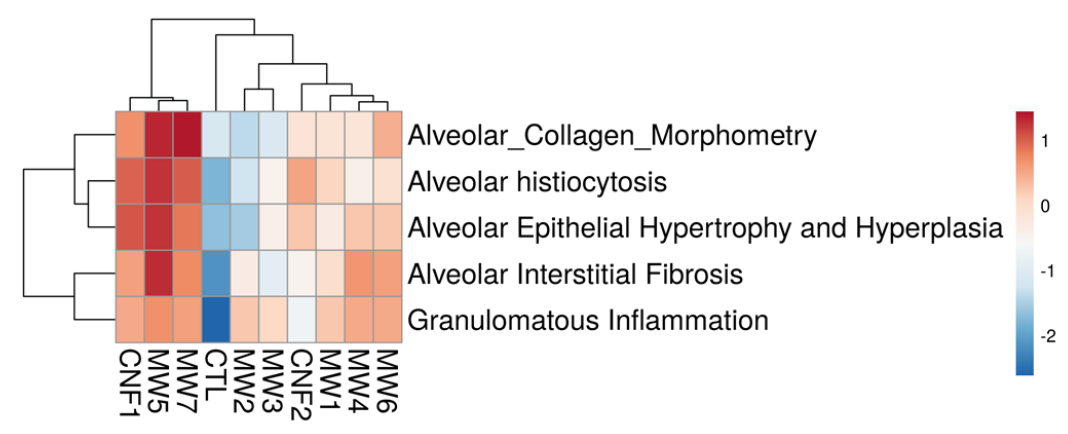

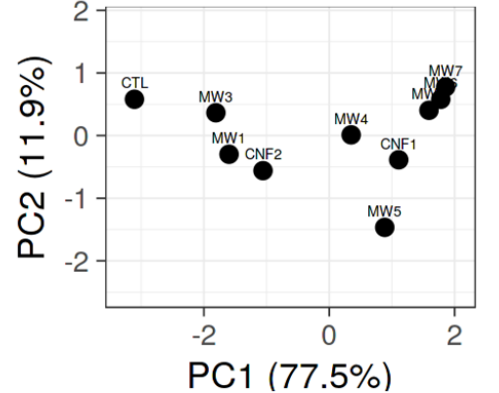

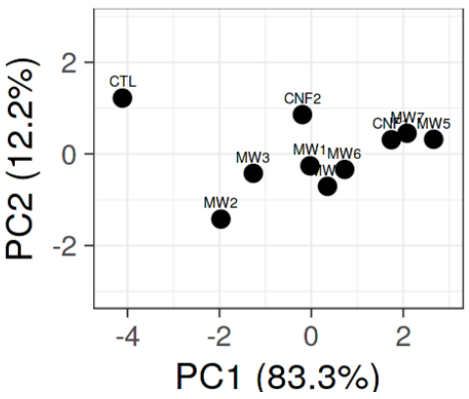

Figure 4.6. Hierarchical clustering analysis (HCA) and principal component analysis (PCA) of different $\mathrm{CNT} / \mathrm{F}$ materials and bronchi/bronchiolar histopathology outcomes (A) and alveolar histopathology outcomes (B). 

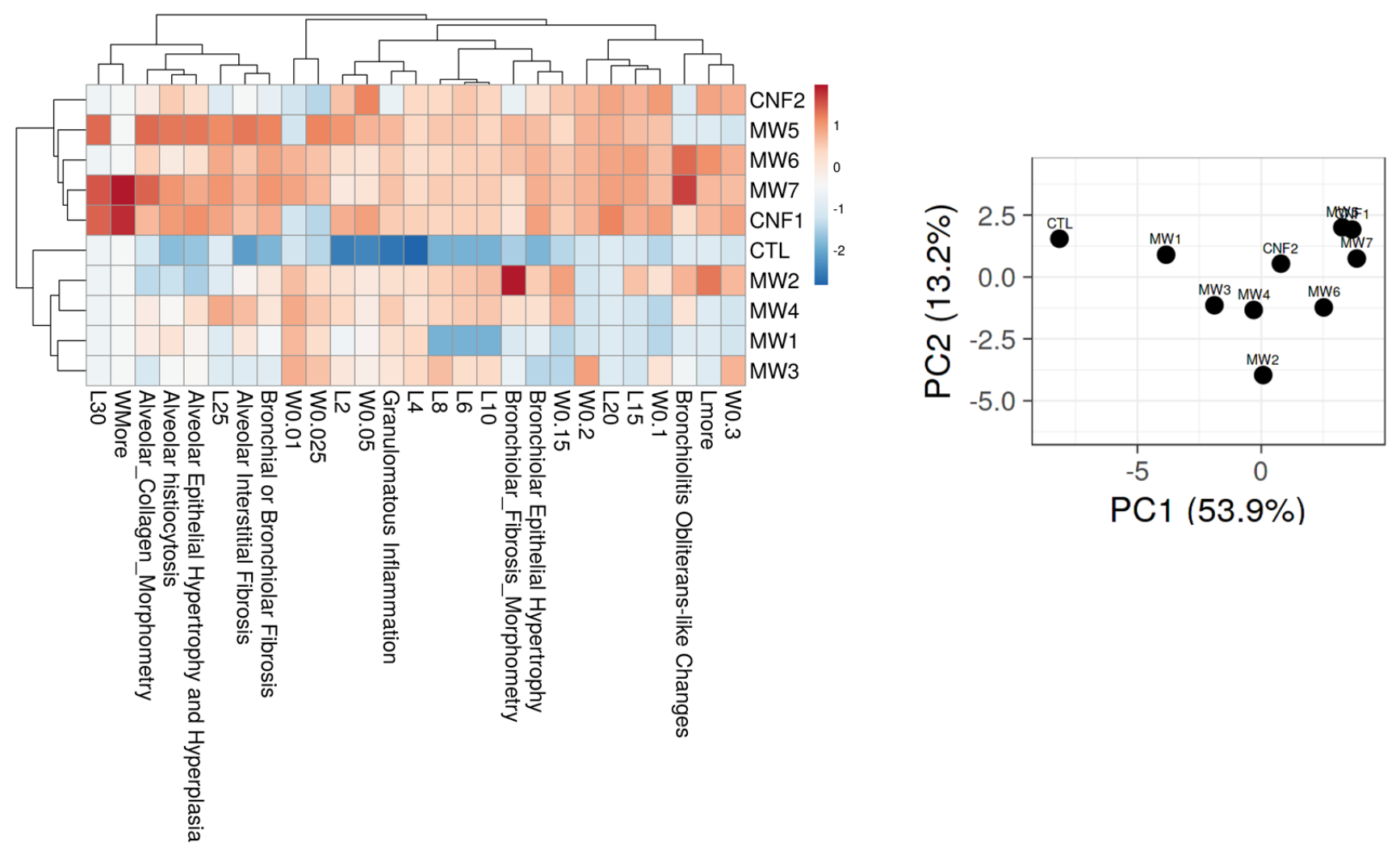

Figure 4.7. Hierarchical clustering analysis (HCA) and principal component analysis (PCA) of different CNT/F materials comparing length - diameter physical dimension (L-W binning) combined with all histopathology outcomes. 

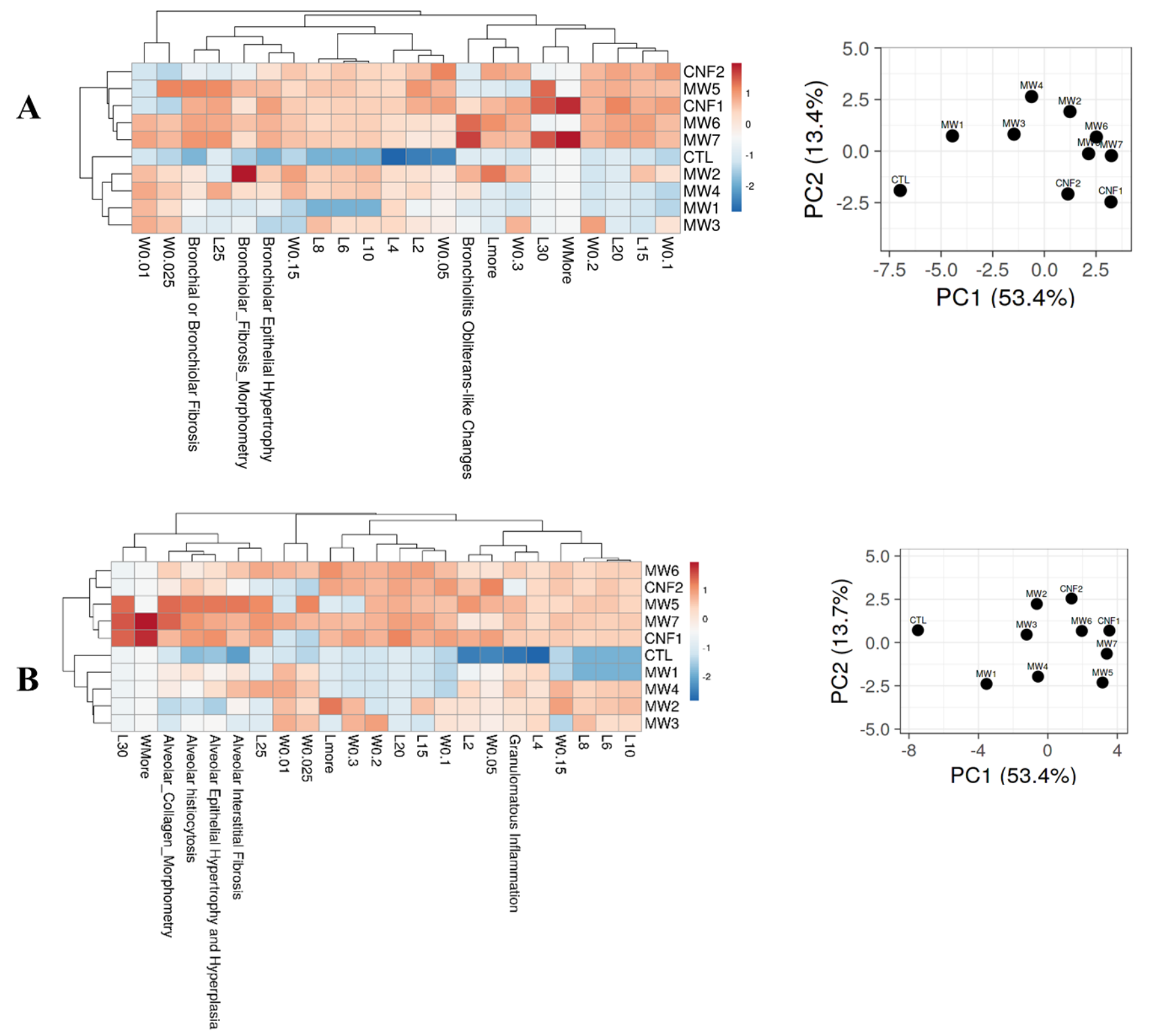

Figure 4.8. Hierarchical clustering analysis (HCA) and principal component analysis (PCA) of different CNT/F materials comparing bronchial/bronchiolar histopathology outcomes (A) and alveolar pathology outcomes (B). 
A

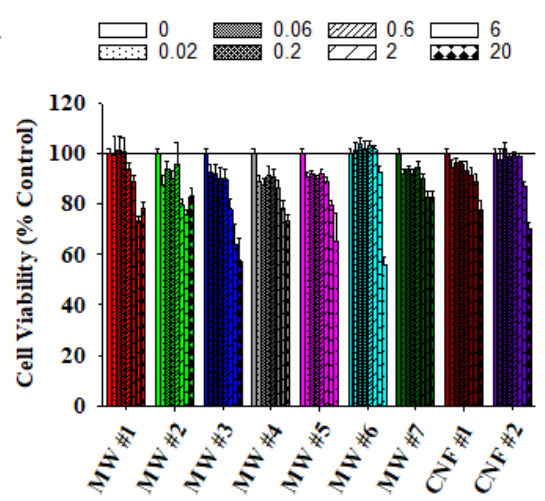

C

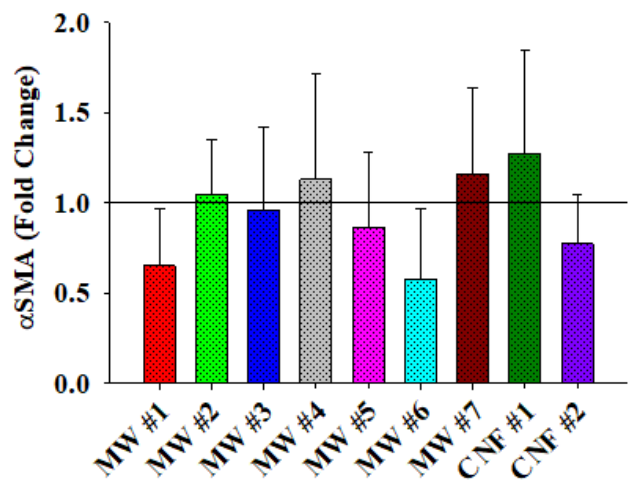

B

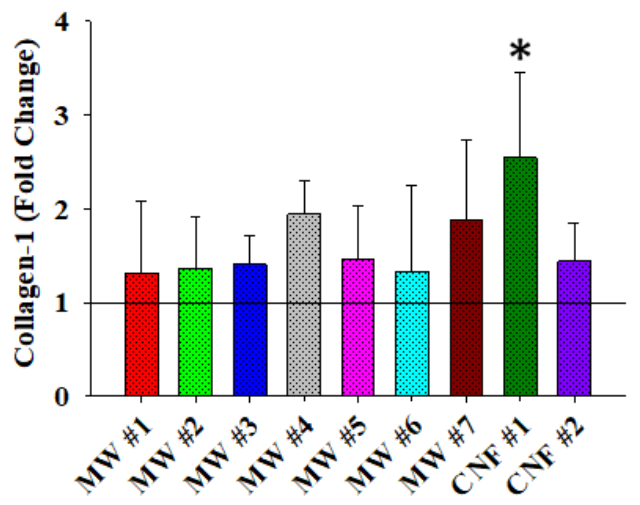

D

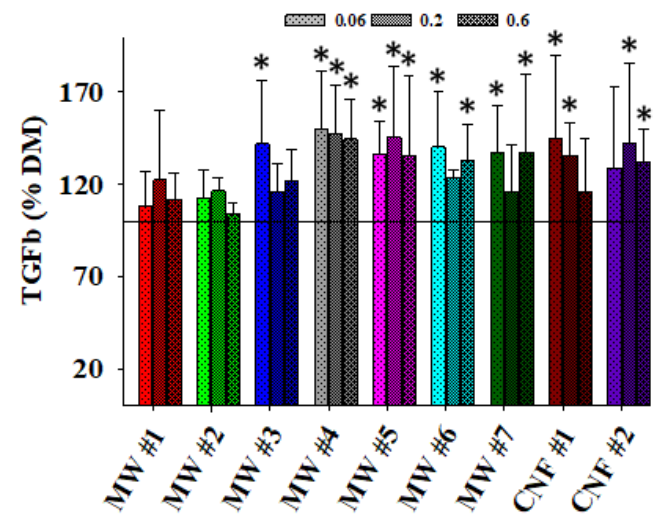

Figure 4.9. Human fibroblast in vitro cell viability (A), collagen-1 production (B), asmooth muscle actin production (C), and TGF $\beta$ secretion (D) following exposure to MWCNT/CNF at $0-20 \mu \mathrm{g} / \mathrm{cm}^{3}$ for 24 hours. Collagen-1 production was significantly increased following exposure to MW \#7. No changes in $\alpha$ smooth muscle actin were seen, and variable changes in TGF $\beta$ secretion were noted with significance. $(* \mathrm{p}<0.05)$ 

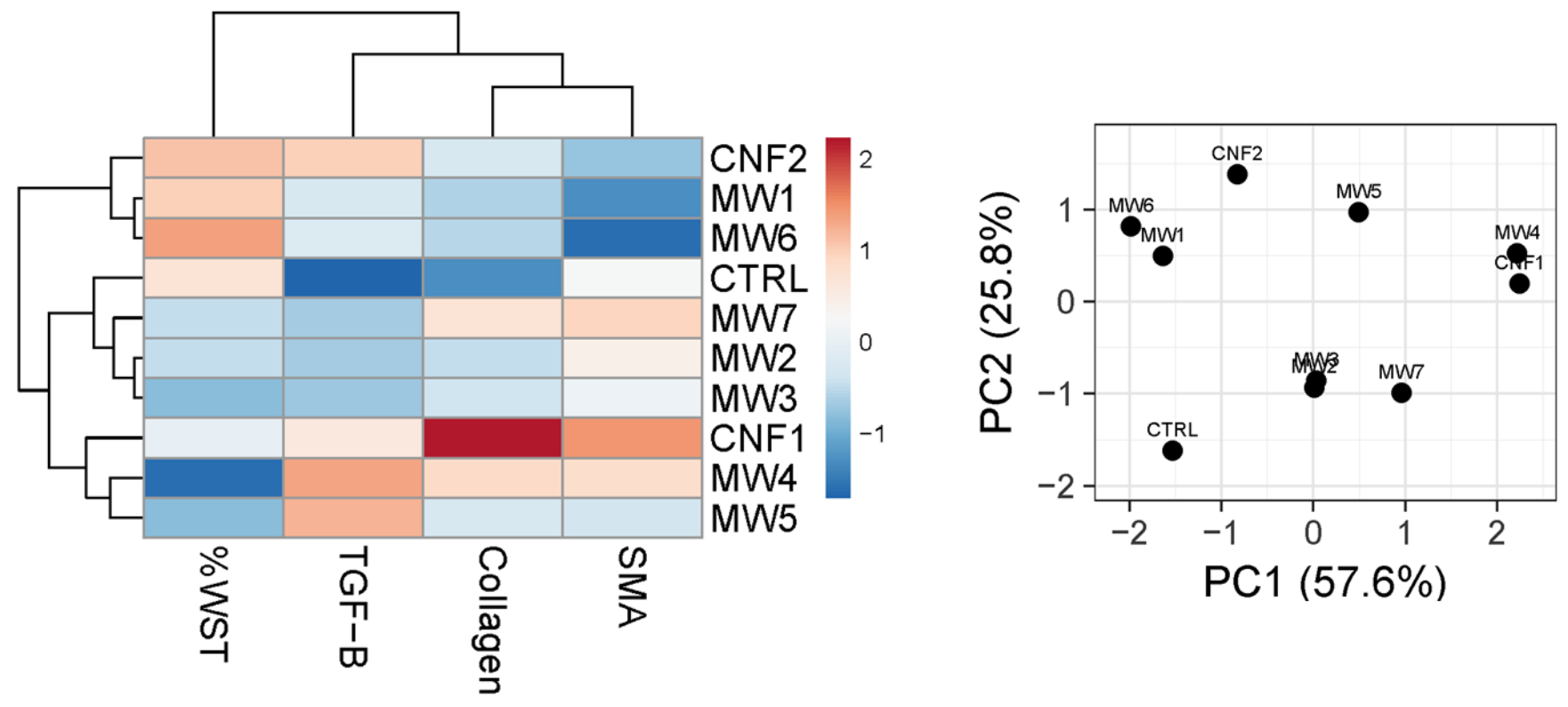

Figure 4.10. Hierarchical clustering analysis (HCA) and principal component analysis (PCA) of different CNT/F materials comparing in vitro fibroblast outcomes. 

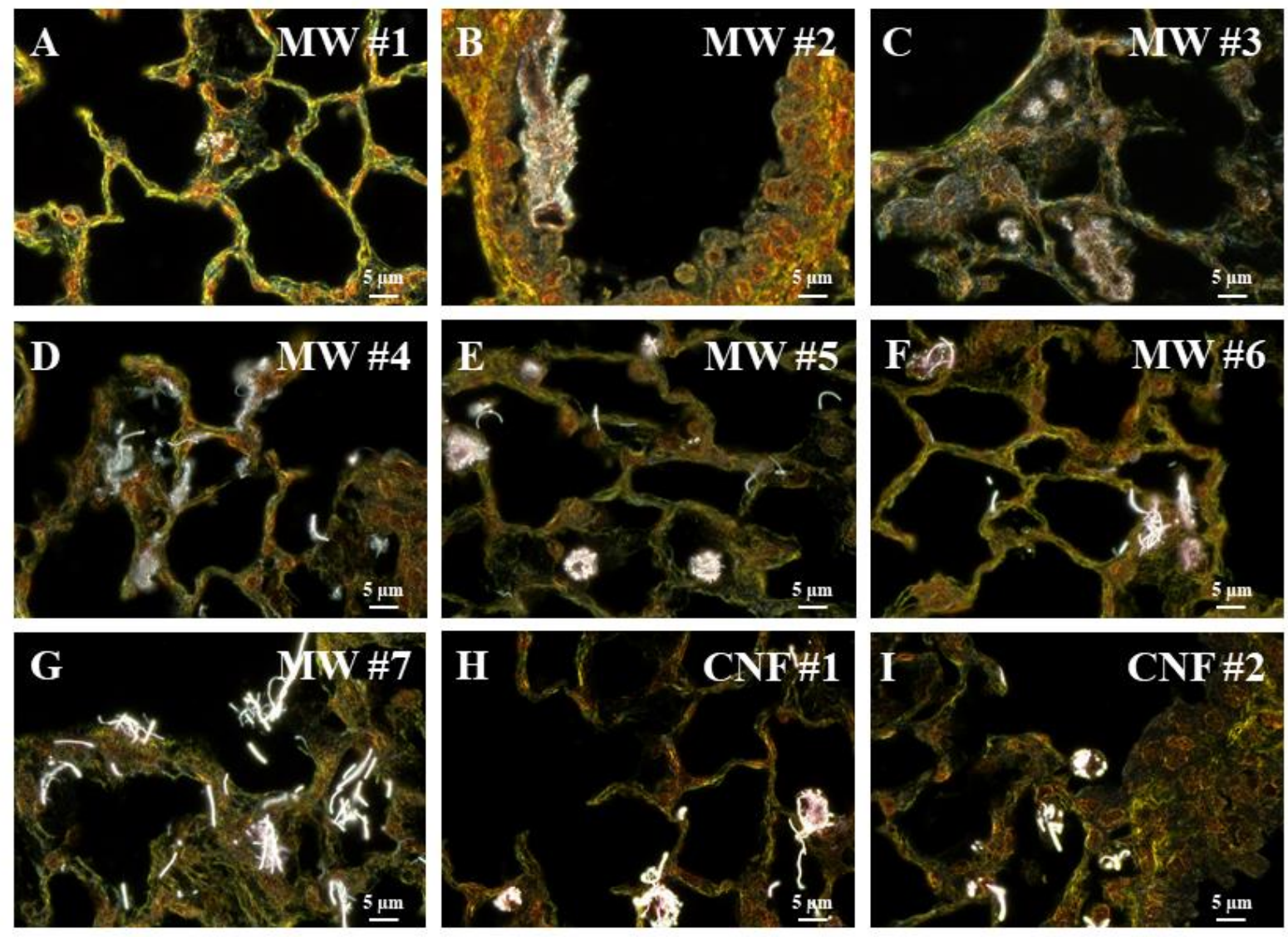

Figure 4.11. Representative Dark Field Micrographs of lungs at 1 Day post-exposure to $40 \mu \mathrm{g}$ MWCNT or CNF. Images A-I are of MW \#1, 2, 3, 4, 5, 6, 7, and CNF \#1, and 2 respectively. MW \#1 and 3 deposition is common in the alveolar region, typically as small spherical agglomerates. MW\#2 deposition is limited to conducting airways as large aggregates. MW \#4-7 and CNF \#1-2 are found as both bundled agglomerates and singlets in the alveolar region. Scale bar is $5 \mu \mathrm{m}$. 

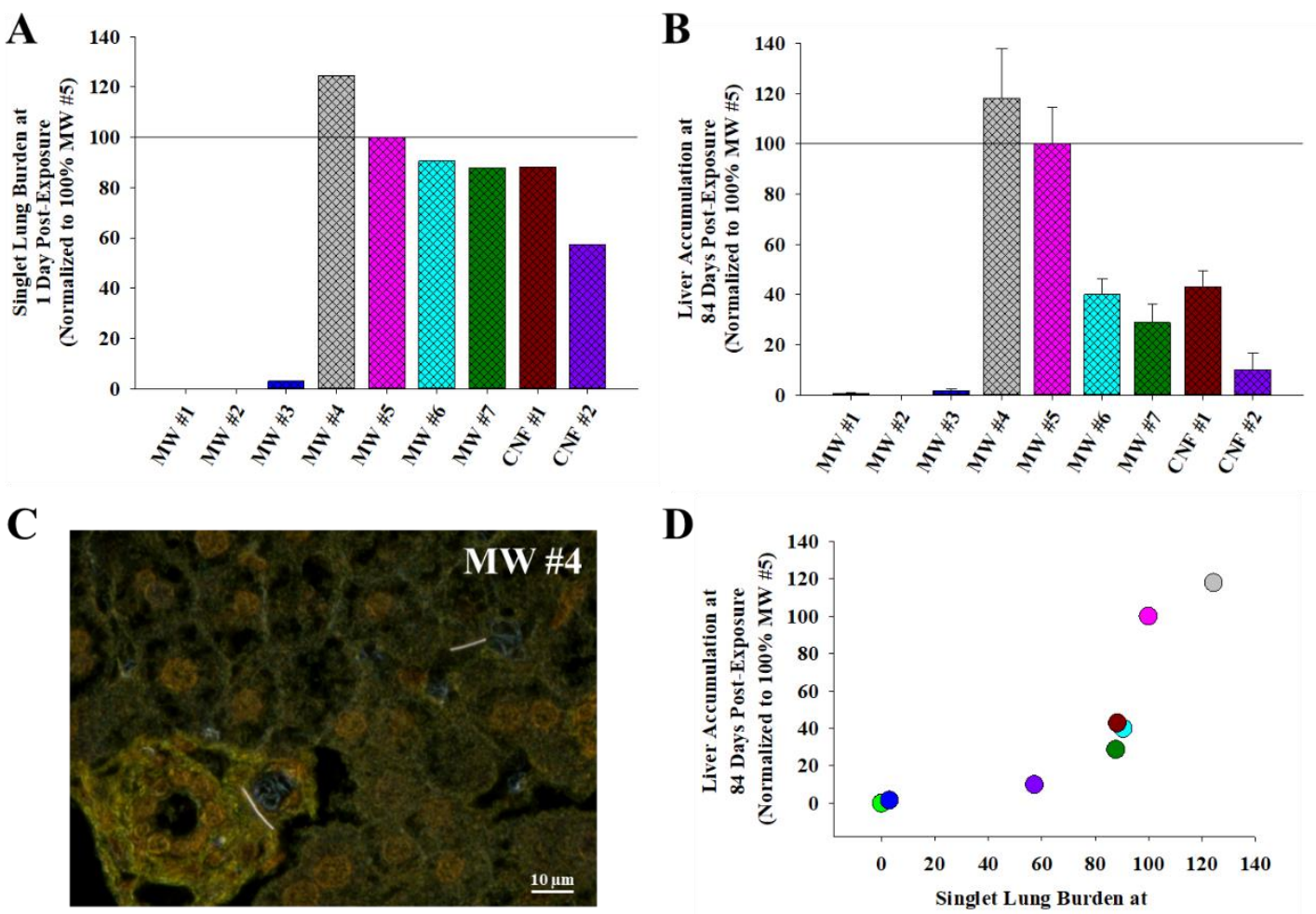

D

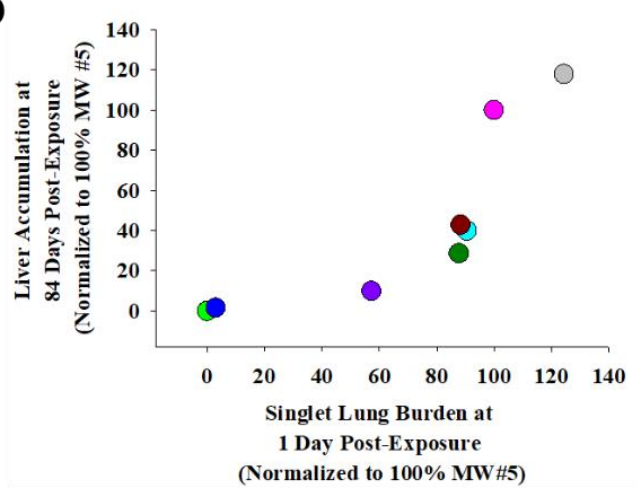

Figure 4.12. Lung singlet burden at $1 \mathrm{~d}$ post exposure predicts extra-pulmonary translocation at $84 \mathrm{~d}$ post-exposure. A) Lung singlet burden was quantified at $1 \mathrm{~d}$ post exposure and is presented as a percentage normalized to $100 \%$ MW \#5. Reference bar indicates 100\%. MW \#1-3 were almost exclusively present as agglomerated material with minimal to no singlet present. MW \#4 had the greatest lung singlet burden, followed by MW \#5. MW \#6, \#7, and CNF \#1 had notable singlet burden with CNF \#2 having a moderate singlet burden. B) Extra-pulmonary translocation to the liver was quantified and is presented as percentage normalized to $100 \%$ MW \#5. Reference bar indicates $100 \%$. MW \#1-3 had none to little translocation to the liver at $84 \mathrm{~d}$ post-exposure. MW \#4 had the greatest, followed by MW \#5. Some translocation of MW \#6, \#7, and CNF \#1 was measured, with CNF \#2 have minimal but notable translocation. C) Representative dark field micrograph of MW \#4 singlet present in the liver at $84 \mathrm{~d}$ post-exposure. Scale bar is $10 \mu \mathrm{m}$. D) Relationship of lung singlet burden at $1 \mathrm{~d}$ postexposure to extra-pulmonary translocation at $84 \mathrm{~d}$ post exposure. Greater presence of singlets in the lung 
at $1 \mathrm{~d}$ post-exposure correlates strongly to greater extra-pulmonary translocation. MW \#4 has the greatest singlet lung burden and the greatest liver translocation followed by MW \#5. Translocated particles in the liver were only observed as singlets. Particles with minimal singlet burden had none to minimal translocation with the moderate singlet burden materials having moderate translocation. 

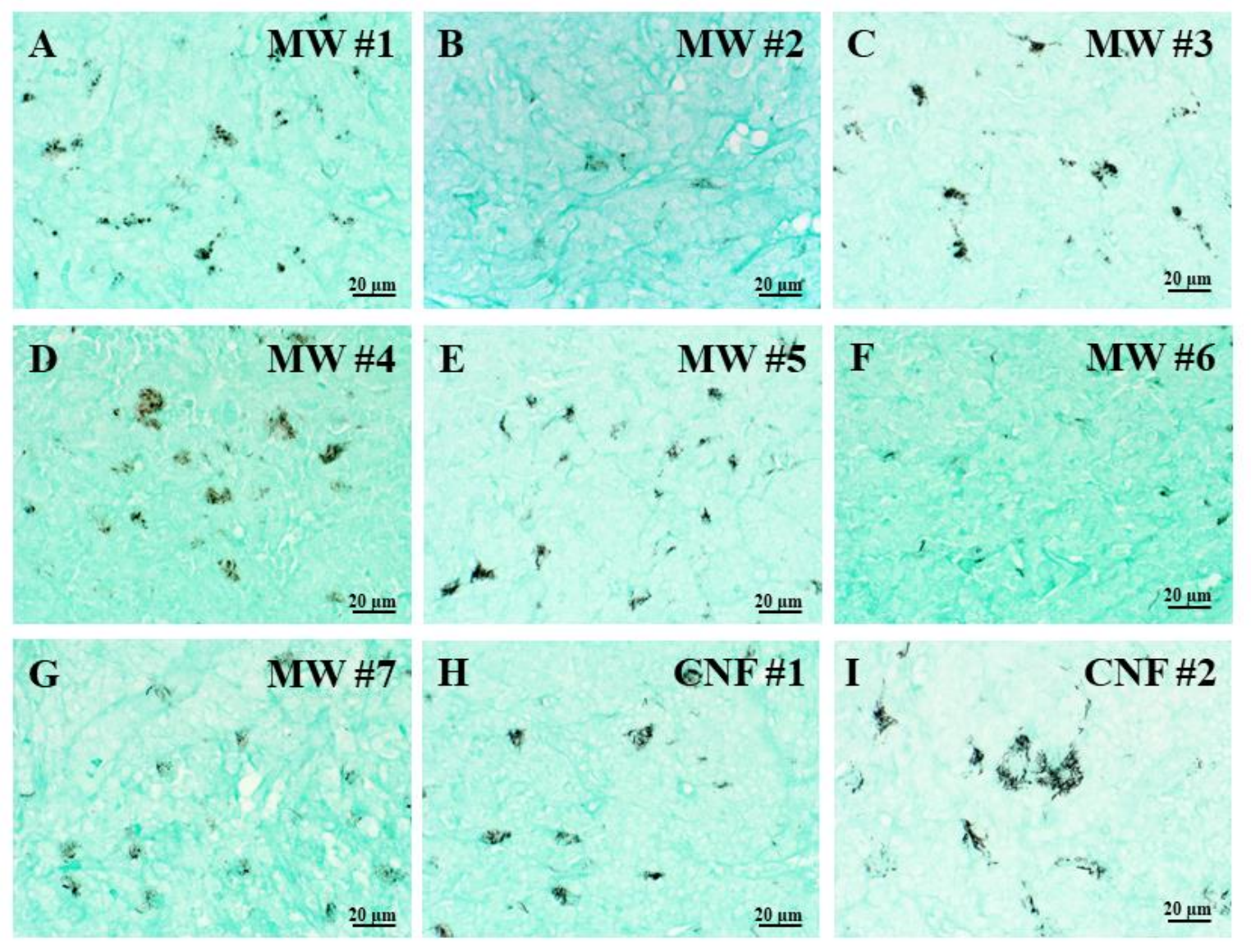

Figure 4.13. Representative Micrographs of Tracheobronchial Lymph Nodes at 84 Days post-exposure to $40 \mu \mathrm{g}$ MWCNT or CNF. Images A-I are of MW \#1, 2, 3, 4, 5, 6, 7, and CNF \#1, and 2 respectively at 60x magnification. Green counterstain was used to visualize black particle presence in the tissue. MW \#1 and MW \#3 had the most prominent translocation, though all materials with the exception of MW \#2 had notable translocation. 

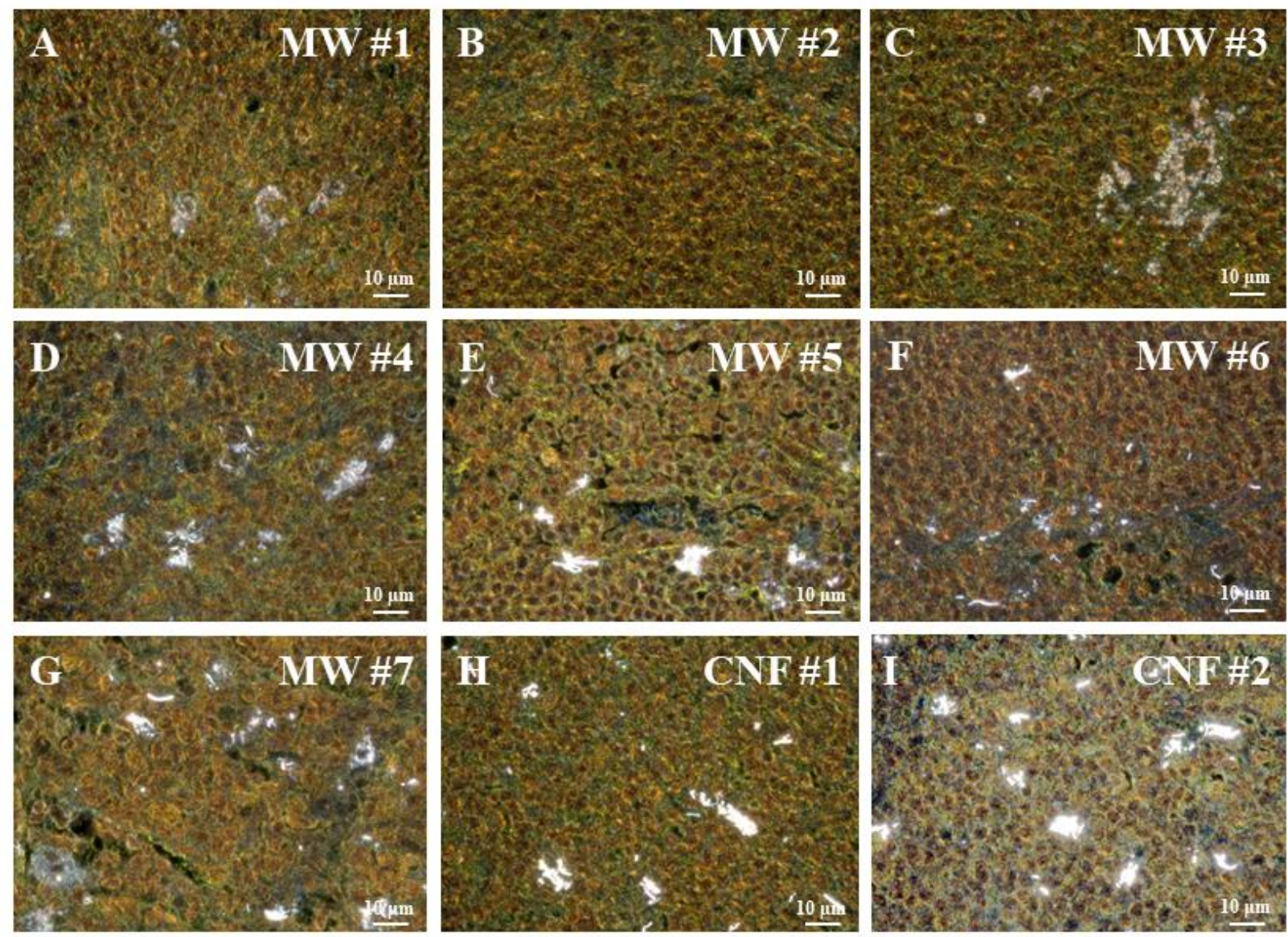

Figure 4.14. Representative Dark Field Micrographs of Tracheobronchial Lymph Nodes at 84 Days postexposure to $40 \mu \mathrm{g}$ MWCNT or CNF. Images A-I are of MW \#1, 2, 3, 4, 5, 6, 7, and CNF \#1, and 2 respectively. Dark field microscopy was used to visualize both agglomerates and singlets present in the tissue. The presence of singlets in MW \#5-7, and CNF \#1-2 can be more easily identified in addition to the presence of agglomerated or accumulated particle. Scale bars represent $10 \mu \mathrm{m}$. 
A
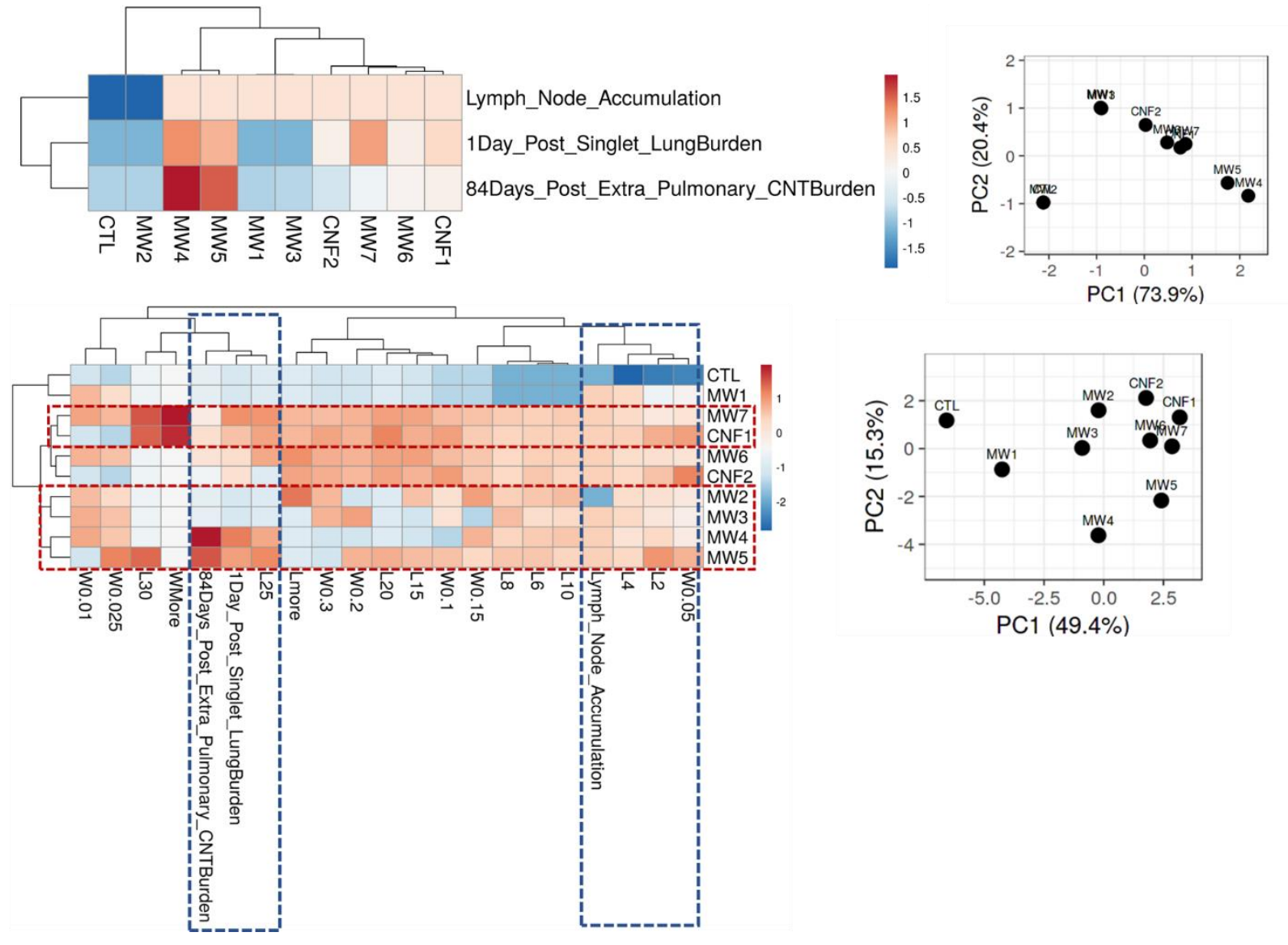

Figure 4.15. Hierarchical clustering analysis (HCA) principal component analysis (PCA) of different $\mathrm{CNT/F}$ materials comparing translocation outcomes alone (A), as well as length - diameter physical dimension (B; L-W binning) with extra-pulmonary translocation outcomes. 


\section{CHAPTER 4 SUPPLEMENTARY TABLES AND FIGURES}

Supplementary Table 4.1. Physical dimensions of CNT/F dispersed in isopropanol.

\begin{tabular}{|c|c|c|c|c|c|c|c|c|c|c|}
\hline & & MW \#1 & MW \#2 & MW \#3 & MW \#4 & MW \#5 & MW \#6 & MW \#7 & CNF \#1 & CNF \#2 \\
\hline \multirow{6}{*}{$\begin{array}{l}\text { Diameter } \\
(\mathrm{nm})\end{array}$} & $\begin{array}{r}\text { Company Reported } \\
\text { Diameter (nm) }\end{array}$ & $6-9$ & 10 & $10-15$ & $5-30$ & N/A & $70-80$ & 150 & 100 & 150 \\
\hline & $\begin{array}{r}\text { Geometric Mean } \\
(\mathrm{nm} \pm \mathrm{GSD})\end{array}$ & $13 \pm 1$ & $14 \pm 2$ & $20 \pm 2$ & $19 \pm 1$ & $63 \pm 1$ & $28 \pm 2$ & $37 \pm 2$ & $102 \pm 1$ & $103 \pm 1$ \\
\hline & $\begin{array}{r}\text { Arithmetic Mean } \\
(\mathrm{nm} \pm \mathrm{SE})\end{array}$ & $13 \pm 0$ & $16 \pm 1$ & $26 \pm 2$ & $20 \pm 1$ & $67 \pm 2$ & $38 \pm 3$ & $54 \pm 4$ & $110 \pm 3$ & $110 \pm 3$ \\
\hline & Median & 12 & 14 & 19 & 18 & 63 & 25 & 28 & 98 & 100 \\
\hline & Diameter Range & 6-29 & $6-216$ & $8-275$ & $8-133$ & $21-168$ & $8-218$ & $9-425$ & $40-397$ & $46-263$ \\
\hline & $\begin{array}{r}\text { Normal } \\
\text { Distribution } \\
\end{array}$ & Lognormal & & & & & & & & Lognormal \\
\hline \multirow{6}{*}{ Length $(\mu \mathrm{m})$} & $\begin{array}{r}\text { Company Reported } \\
\text { Length }(\mu \mathrm{m})\end{array}$ & 5 & N/A & $0.1-10$ & 100 & N/A & $\mathrm{N} / \mathrm{A}$ & N/A & $50-200$ & $50-200$ \\
\hline & $\begin{array}{r}\text { Geometric Mean } \\
(\mu \mathrm{m} \pm \mathrm{GSD})\end{array}$ & $0.67 \pm 1.81$ & $1.34 \pm 2.21$ & $1.10 \pm 2.00$ & $1.41 \pm 1.97$ & $4.39 \pm 2.07$ & $2.05 \pm 2.53$ & $2.88 \pm 4.26$ & $3.64 \pm 2.36$ & $2.16 \pm 2.31$ \\
\hline & $\begin{array}{r}\text { Arithmetic Mean } \\
(\mu \mathrm{m} \pm \mathrm{SE})\end{array}$ & $0.80 \pm 0.03$ & $1.79 \pm 0.10$ & $1.28 \pm 0.07$ & $1.84 \pm 0.13$ & $5.62 \pm 0.29$ & $3.42 \pm 0.37$ & $7.64 \pm 0.78$ & $5.23 \pm 0.36$ & $3.20 \pm 0.28$ \\
\hline & Median & 0.6607 & 1.5437 & 1.0148 & 1.2896 & 4.547 & 2.1503 & 2.3781 & 3.7273 & 2.0003 \\
\hline & Length Range & $0.1-3.6$ & $0.2-50.9$ & $0.1-8.5$ & $0.3-20.6$ & $1.2-25.8$ & $0.3-37.3$ & $0.1-49.1$ & $0.3-37.6$ & $0.4-42.7$ \\
\hline & $\begin{array}{r}\text { Normal } \\
\text { Distribution } \\
\end{array}$ & Lognormal & Lognormal & Lognormal & & & Lognormal & & Lognormal & Lognormal \\
\hline Aspect Ratio & $\begin{array}{r}\text { Aspect Ratio } \\
(\text { GeoMean } \pm \text { GSD }) \\
\end{array}$ & $53 \pm 2$ & $96 \pm 2$ & $50 \pm 2$ & $76 \pm 2$ & $69 \pm 2$ & $73 \pm 2$ & $78 \pm 3$ & $36 \pm 2$ & $21 \pm 2$ \\
\hline
\end{tabular}


Supplementary Table 4.2. Hydrodynamic diameter, zeta potential, and two-dimensional sizing of CNT/F agglomerates dispersed in physiologic dosing media.

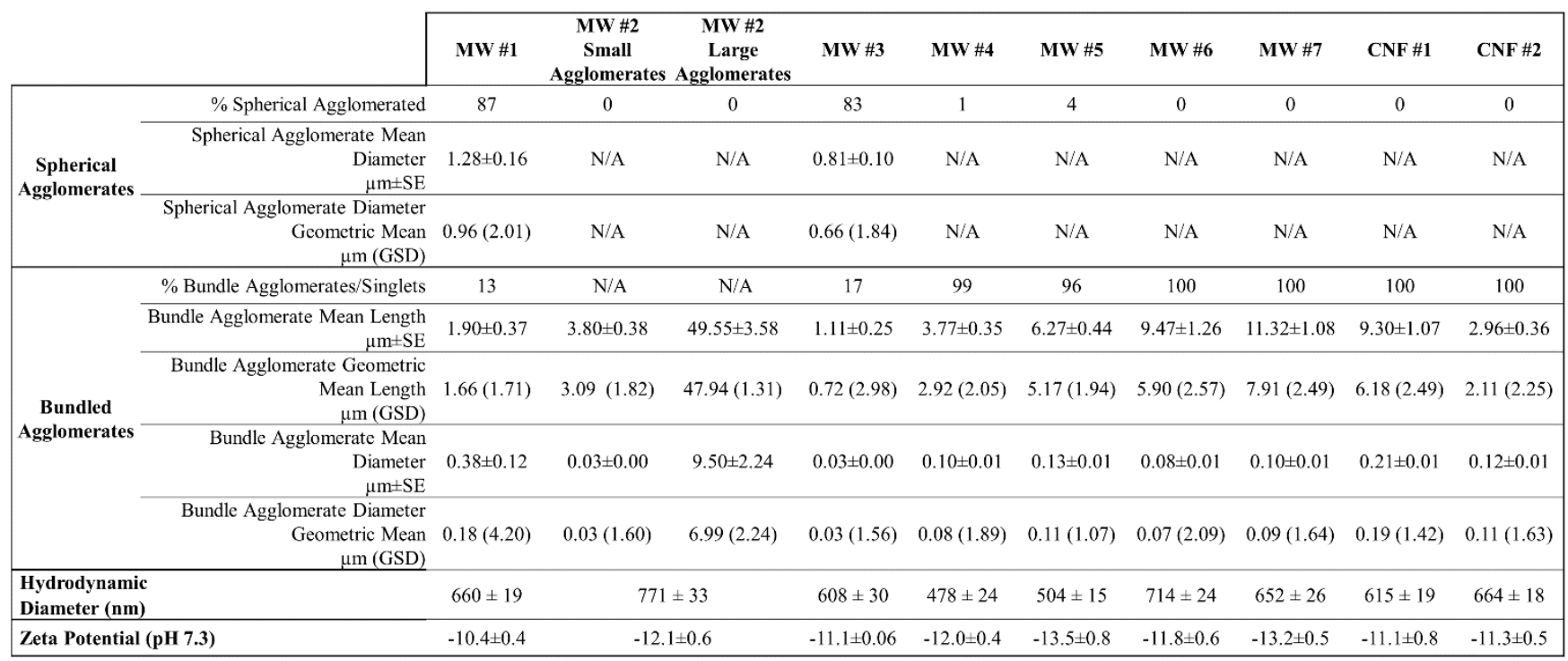


Supplementary Table 4.3. Results of additional particle characterization of CNT/F.

\begin{tabular}{|c|c|c|c|c|c|c|c|c|c|c|}
\hline & & MW \#1 & MW \#2 & MW \#3 & MW \#4 & MW \#5 & MW \#6 & MW \#7 & CNF \#1 & CNF \#2 \\
\hline $\begin{array}{l}\text { Surface Area } \\
\left(\mathrm{m}^{2} / \mathrm{g} \pm \mathrm{SD}\right)\end{array}$ & & $237.7 \pm 1.0$ & $211.9 \pm 1.8$ & $218.6 \pm 1.2$ & $99.4 \pm 1.1$ & $25.2 \pm 0.4$ & $25.4 \pm 0.4$ & $24.7 \pm 0.4$ & $29.4 \pm 0.2$ & $18.0 \pm 0.2$ \\
\hline \multirow{2}{*}{ Dustiness } & $\begin{array}{r}\text { Dustiness Total } \\
(\%) \\
\end{array}$ & 3.8 & 2.9 & 0.3 & 0.5 & 14.0 & 0.2 & 0.2 & 4.9 & ND \\
\hline & $\begin{array}{r}\text { Dustiness } \\
\text { Respirable (\%) } \\
\end{array}$ & 0.84 & 1.10 & 0.20 & 0.20 & 2.40 & 0.08 & 0.09 & 1.40 & ND \\
\hline \multirow{2}{*}{ Density } & $\begin{array}{r}\begin{array}{r}\text { Bulk Density } \\
(\mathrm{g} / \mathrm{cm} 3)\end{array} \\
\end{array}$ & 0.087 & 0.007 & 0.082 & 0.169 & 0.007 & 0.075 & 0.061 & 0.020 & 0.032 \\
\hline & $\begin{array}{r}\text { Tapped Density } \\
(\mathrm{g} / \mathrm{cm} 3)\end{array}$ & 0.119 & 0.008 & 0.095 & 0.222 & 0.010 & 0.095 & 0.073 & 0.028 & 0.045 \\
\hline Endotoxin & & BLD & BLD & BLD & BLD & BLD & BLD & BLD & BLD & BLD \\
\hline PAH & & BLD & BLD & BLD & BLD & BLD & BLD & BLD & BLD & BLD \\
\hline \multirow{2}{*}{ Metal Catalyst } & $\% \mathrm{Fe}$ & 0.317 & 1.725 & 1.603 & 3.423 & 0.270 & 5.006 & 6.169 & 1.168 & 1.142 \\
\hline & $\% \mathrm{Al}$ & 0.310 & 0.028 & 2.116 & 0.019 & N/A & 0.035 & $\mathrm{~N} / \mathrm{A}$ & 0.006 & 0.013 \\
\hline \multirow{2}{*}{ TGA } & $\begin{array}{r}\text { TGA -Avg onset } \\
\text { oxidation, }{ }^{\circ} \mathrm{C} \\
\end{array}$ & $550 \pm 2$ & $603 \pm 2$ & $575 \pm 0$ & $560 \pm 2$ & $735 \pm 2$ & $581 \pm 0$ & $592 \pm 0$ & $593 \pm 0$ & $694 \pm 0$ \\
\hline & $\begin{array}{r}\text { TGA - Mean } \\
\text { Residual Ash, \% } \\
\end{array}$ & $1.74 \pm 0.01$ & $3.98 \pm 0.26$ & $8.21 \pm 0.26$ & $4.75 \pm 0.07$ & $1.11 \pm 0.28$ & $7.88 \pm 0.15$ & $8.95 \pm 0.29$ & $1.79 \pm 0.12$ & $2.21 \pm 0.16$ \\
\hline $\begin{array}{l}\text { Anti-oxidative } \\
\text { Capacity }\end{array}$ & $\%$ & $64.53 \pm 23.91$ & $75.41 \pm 25.66$ & $76.34 \pm 27.37$ & $88.17 \pm 28.40$ & $91.49 \pm 21.37$ & $84.78 \pm 25.74$ & $77.27 \pm 19.96$ & $100.12 \pm 22.69$ & $99.80 \pm 24.19$ \\
\hline
\end{tabular}




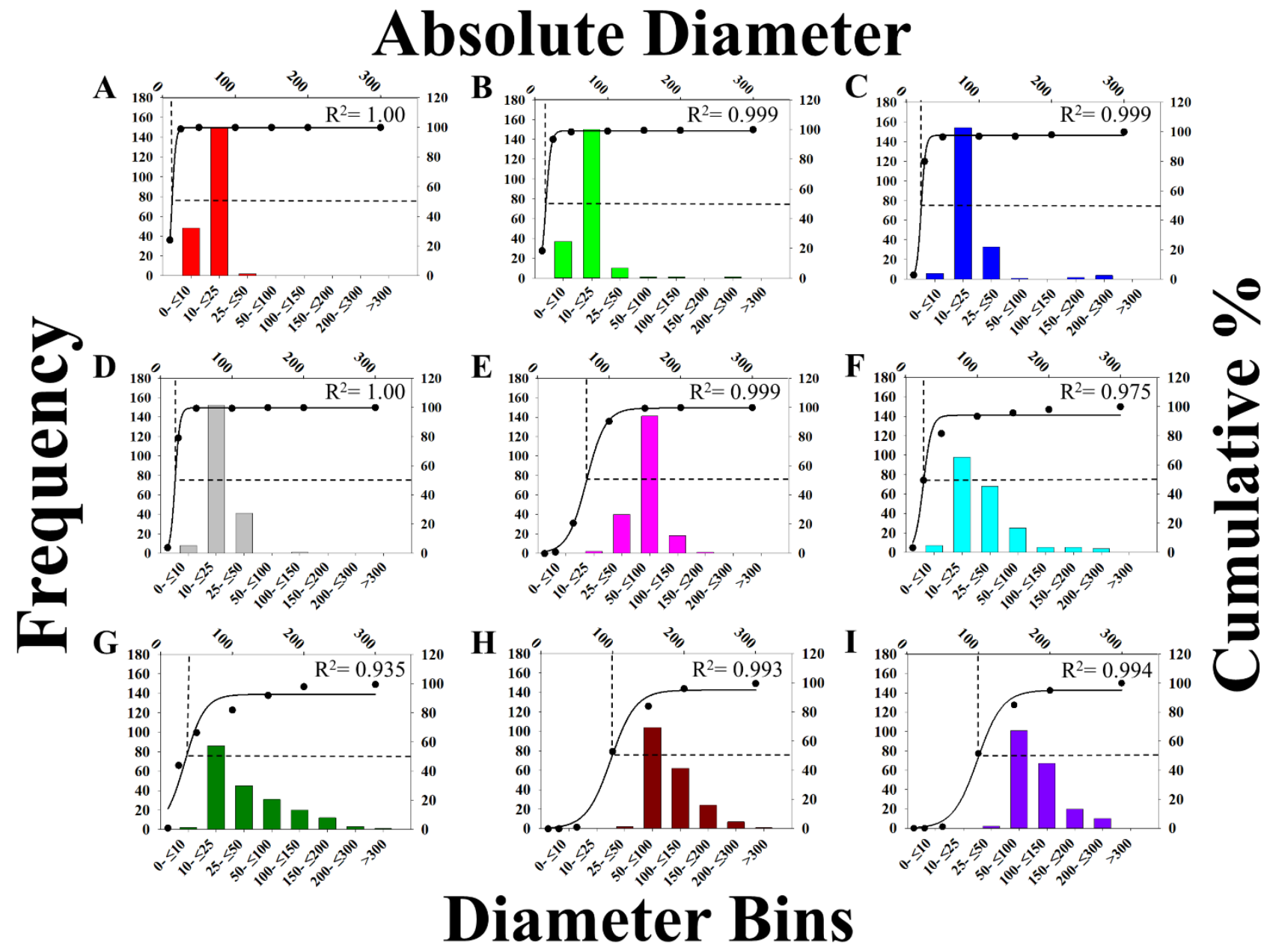

$\square$ MW \#1 MW \#2 MW \#3 MW \#4 MW \#5 MW \#6

Supplementary Figure 4.1. Distributions of CNT/F diameter. 


\section{Absolute Length}
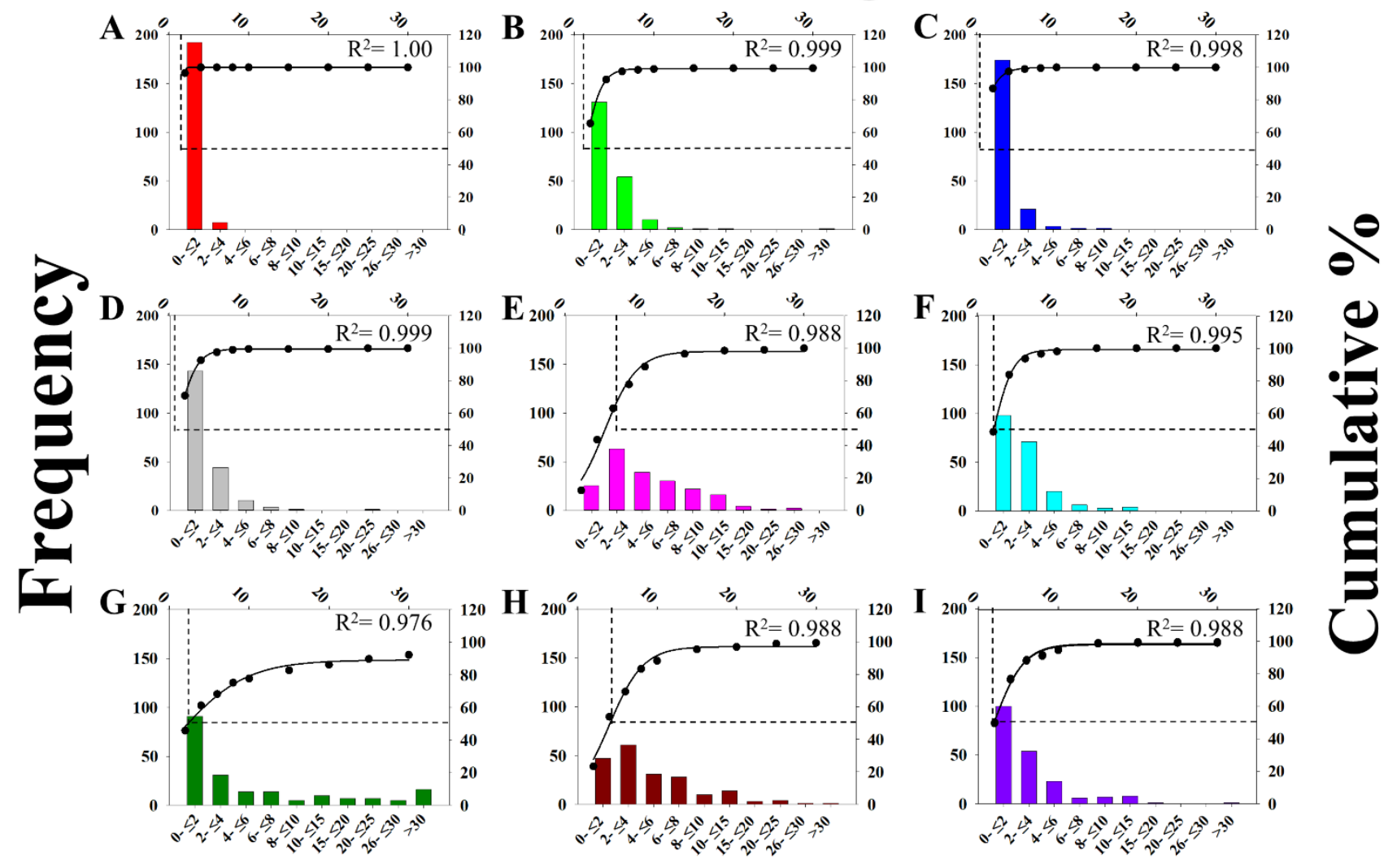

\section{Length Bins}

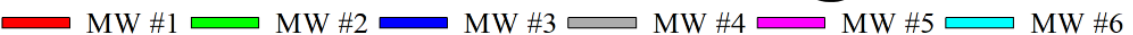

MW \#7

Supplementary Figure 4.2. Distributions of CNT/F length. 

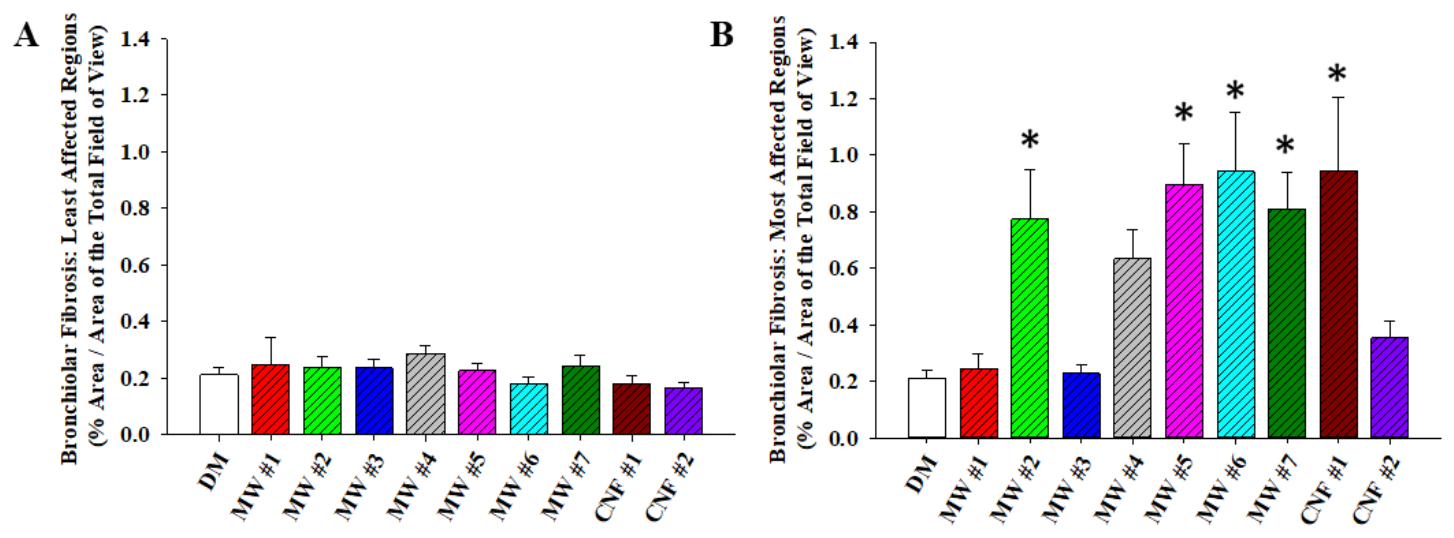

Supplementary Figure 4.3. Bronchial/bronchiolar fibrosis as a percentage of the area of fibrosis over the total area of the field of view for least affected and most affected bronchoalveolar junctions 


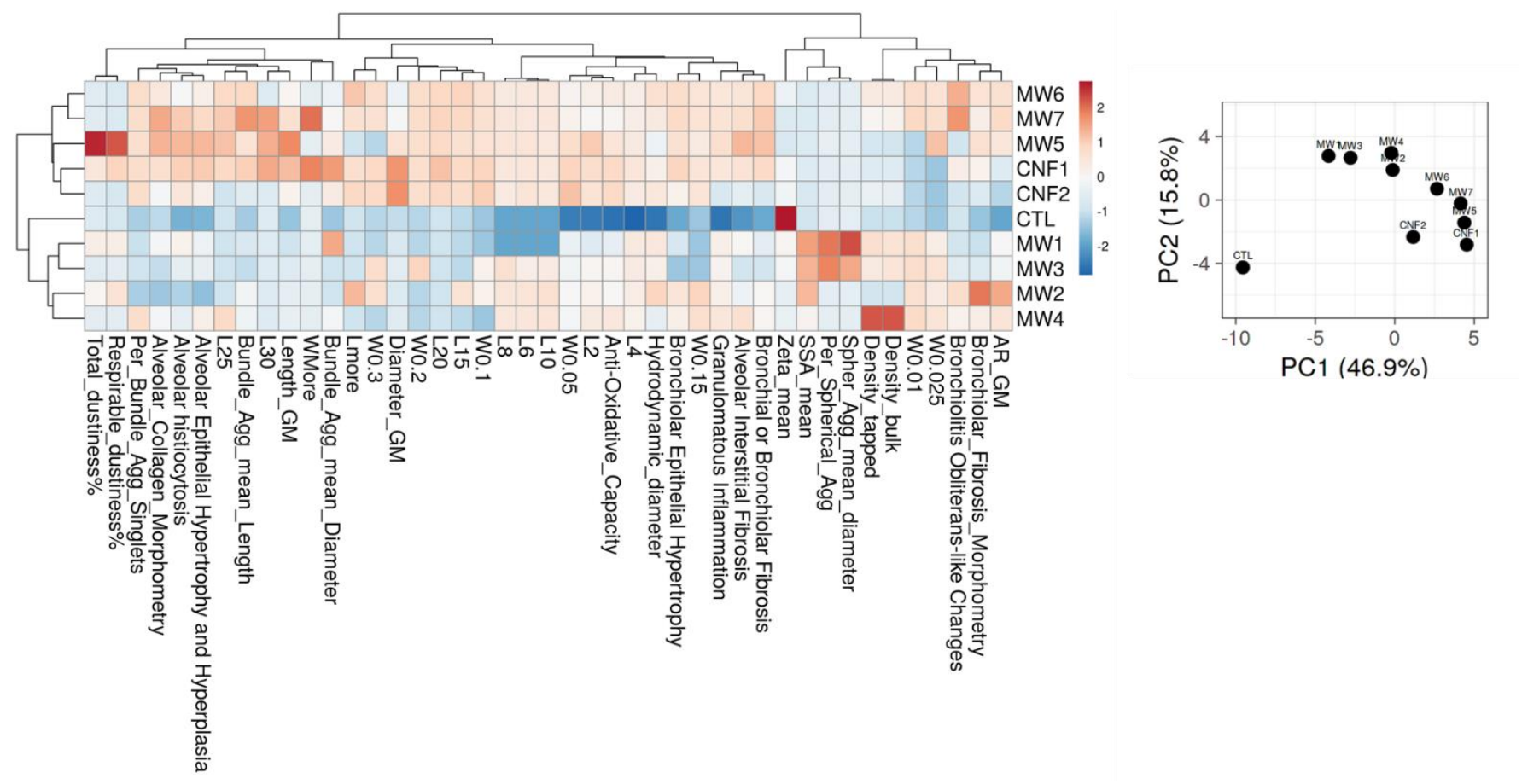

Supplementary Figure 4.4. Hierarchical clustering analysis (HCA) and principal component analysis (PCA) of 'All characterization' and all histopathology outcomes 

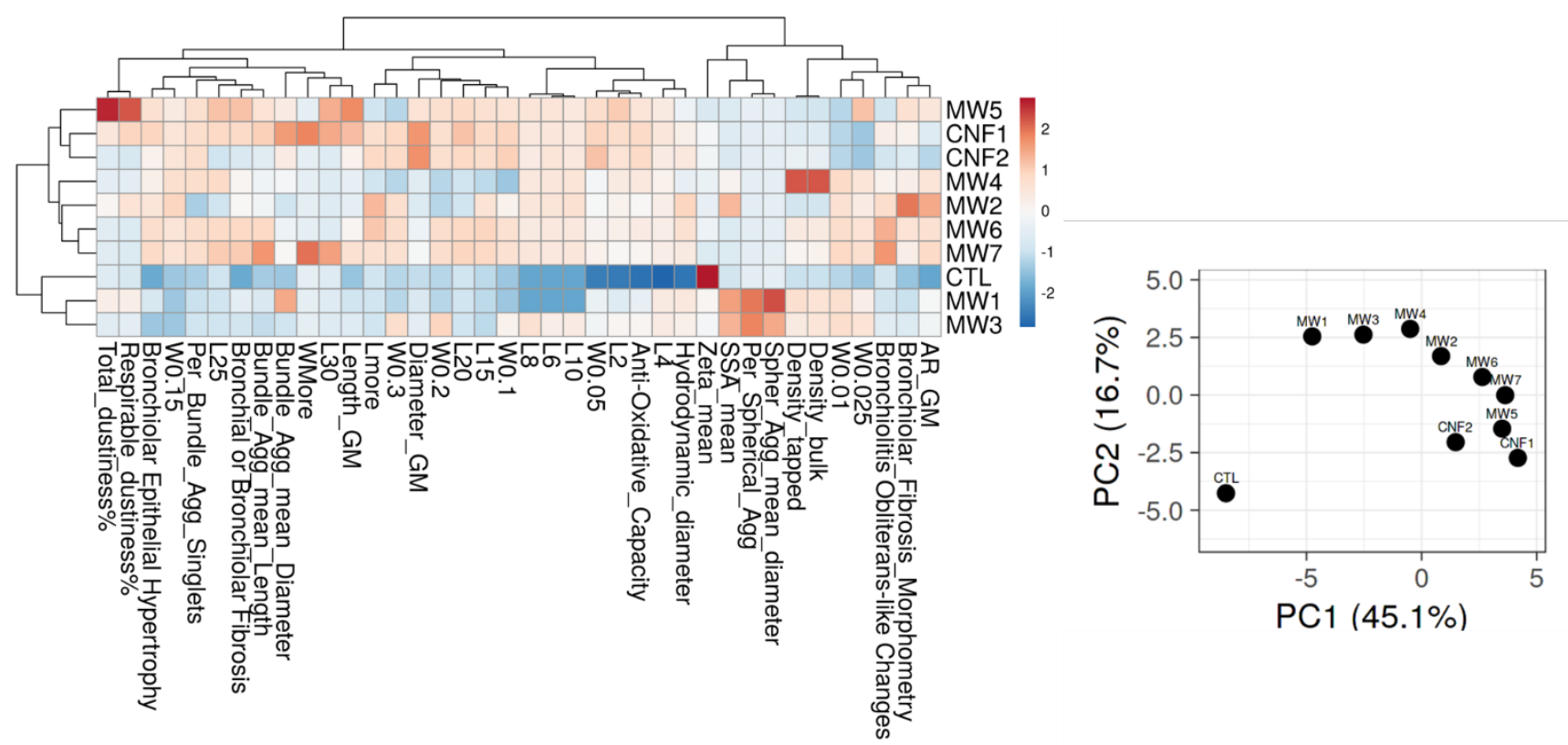

Supplementary Figure 4.5. Hierarchical clustering analysis (HCA) and principal component analysis (PCA) of 'All characterization' and all bronchi-bronchiolar outcomes 


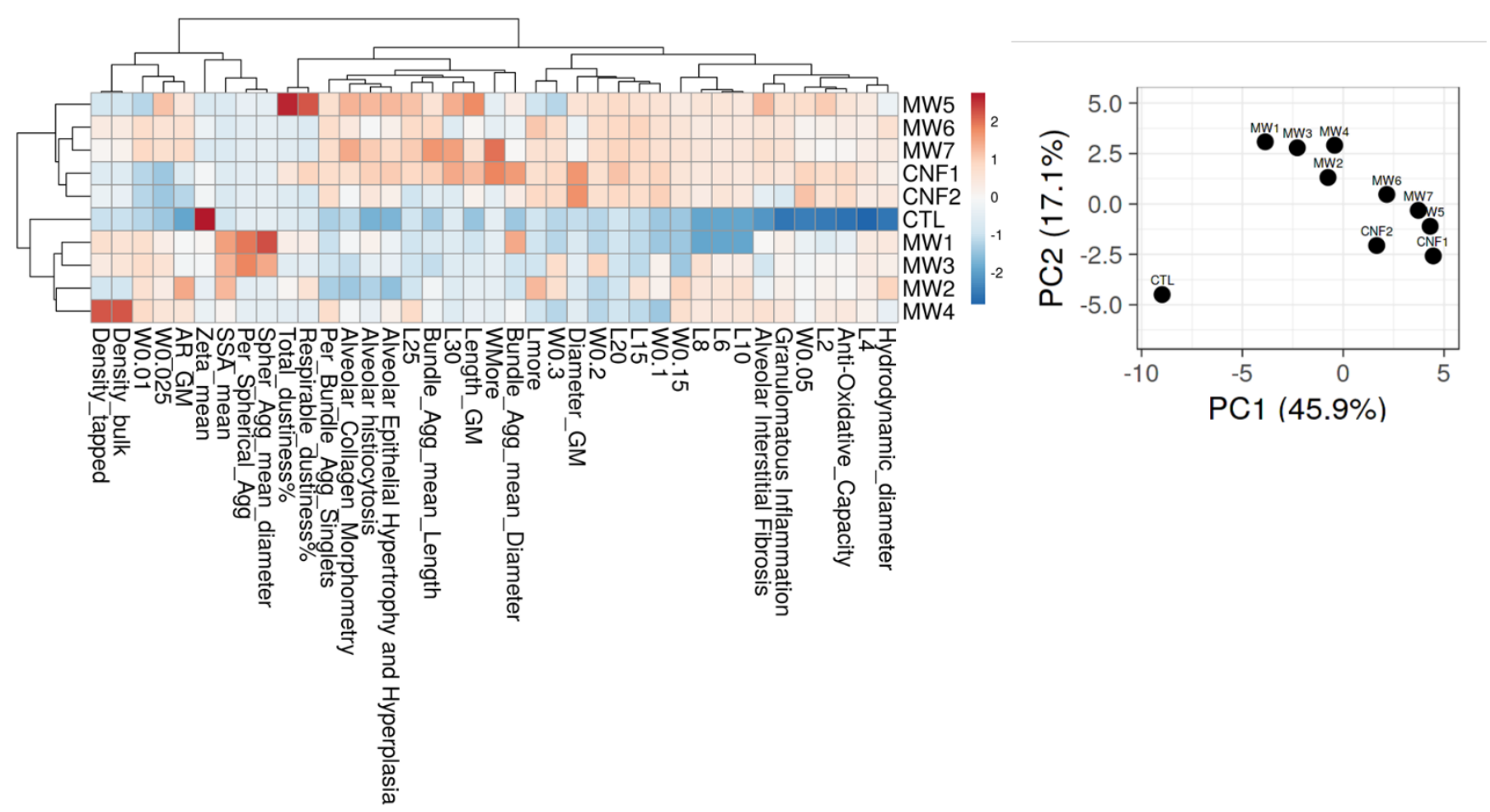

Supplementary Figure 4.6. Hierarchical clustering analysis (HCA) and principal component analysis (PCA) of 'All characterization' and alveolar outcomes 

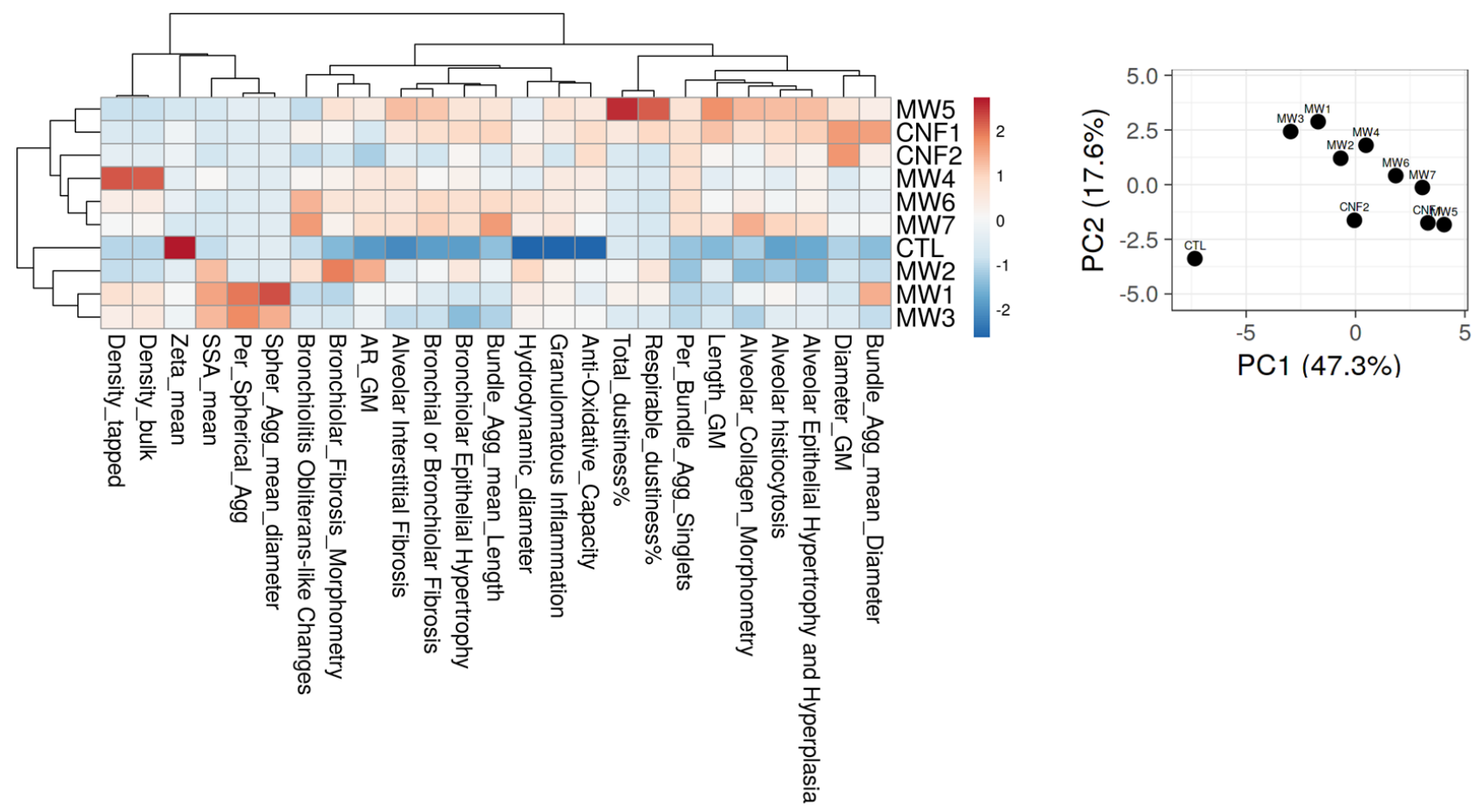

Supplementary Figure 4.7. Hierarchical clustering analysis (HCA) and principal component analysis (PCA) of 'means only' and all histopathology outcomes 

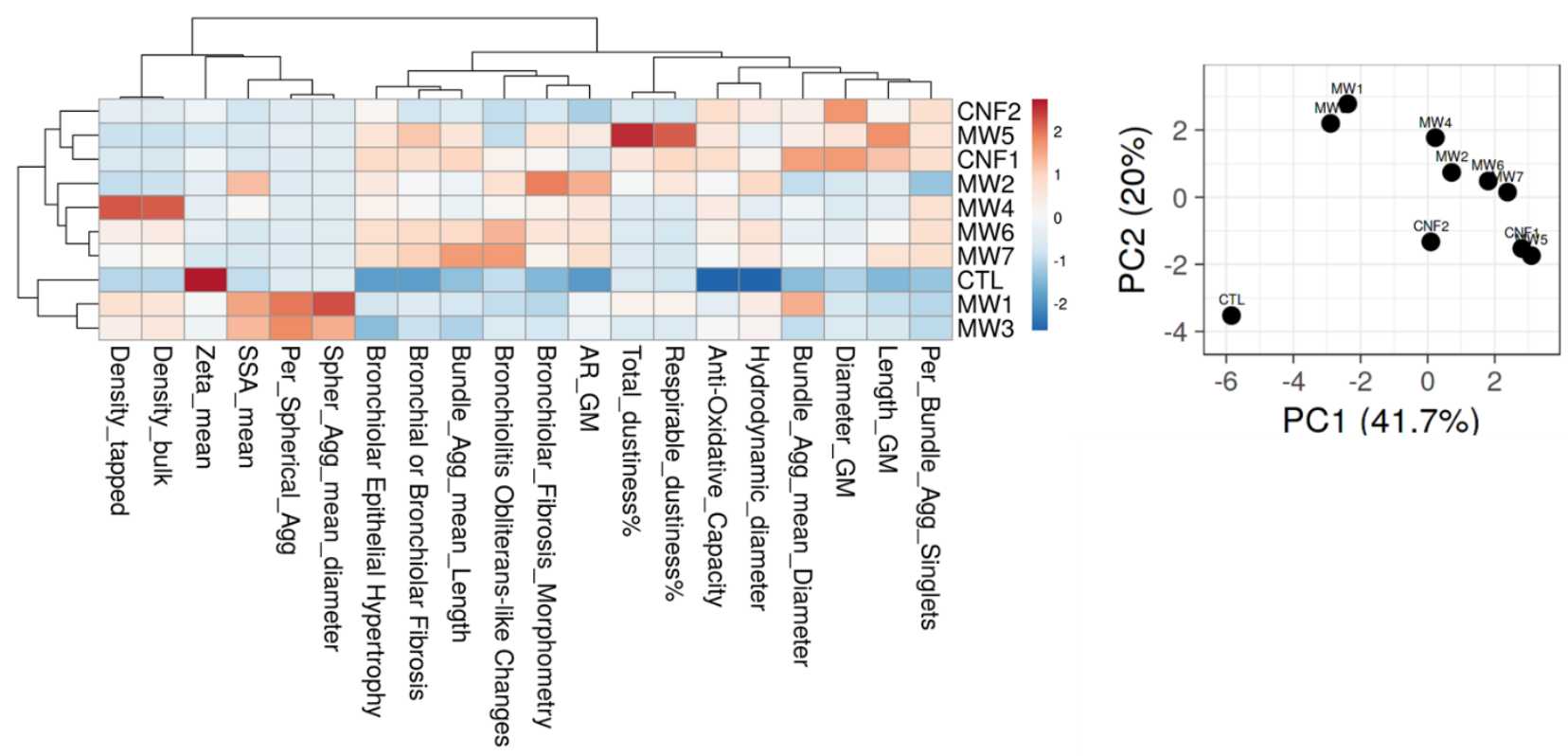

Supplementary Figure 4.8. Hierarchical clustering analysis (HCA) and principal component analysis (PCA) of 'means only' and bronchi/bronchiolar outcomes 

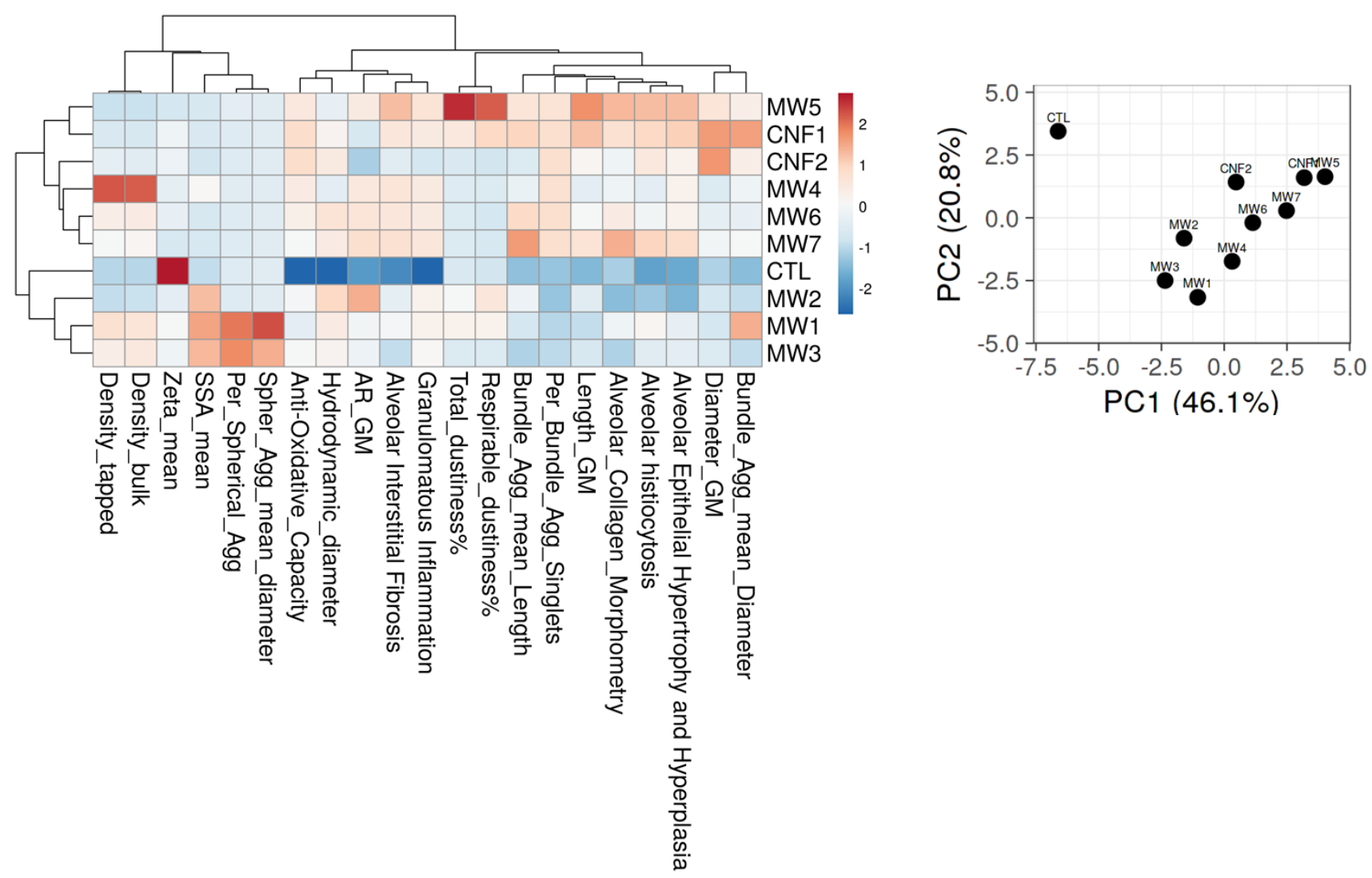

Supplementary Figure 4.9. Hierarchical clustering analysis (HCA) and principal component analysis (PCA) of 'means only' and alveolar outcomes 

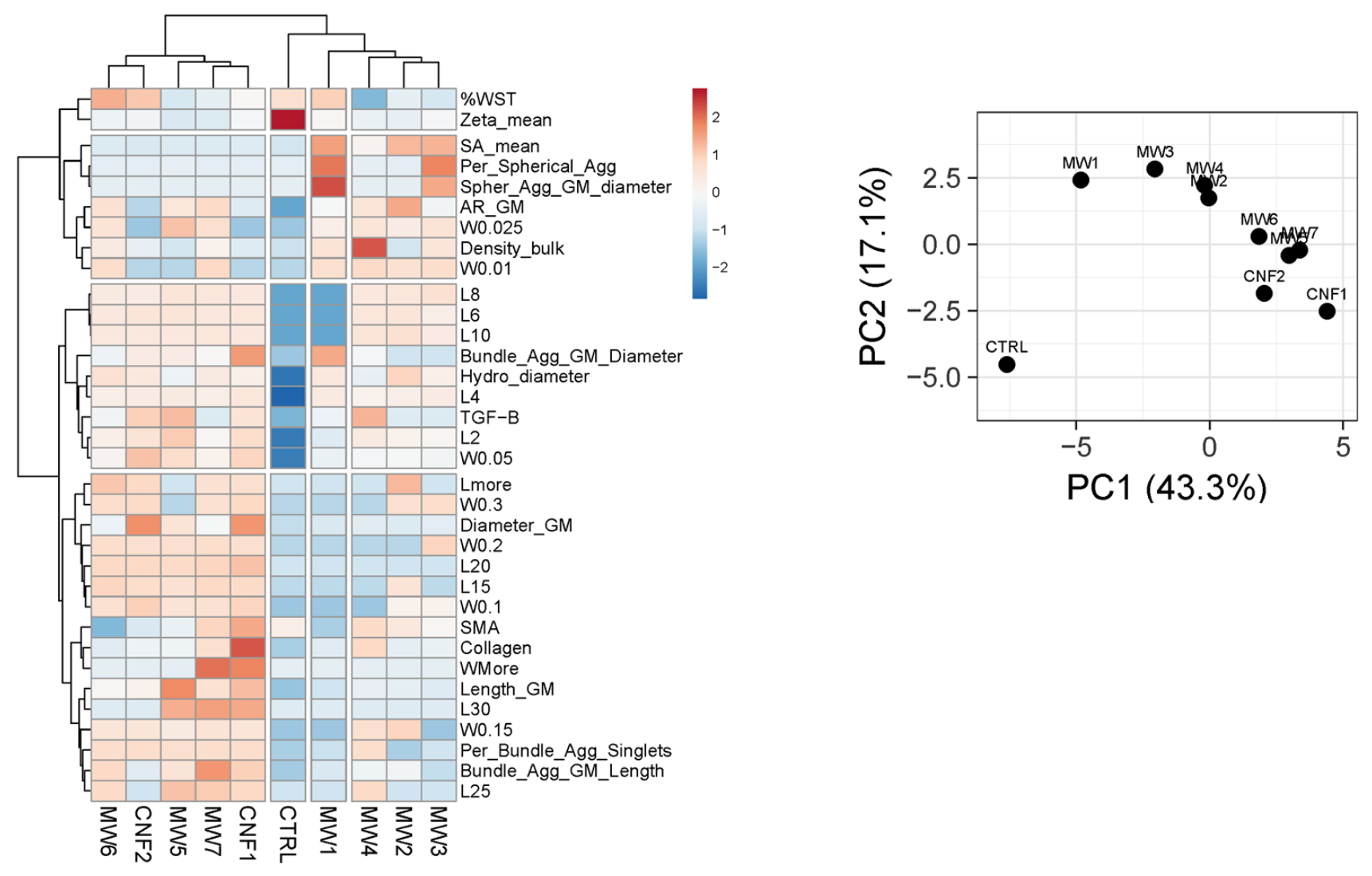

Supplementary Figure 4.10. Hierarchical clustering analysis (HCA) and principal component analysis (PCA) of 'all characterization' and in vitro fibroblast outcomes 
A

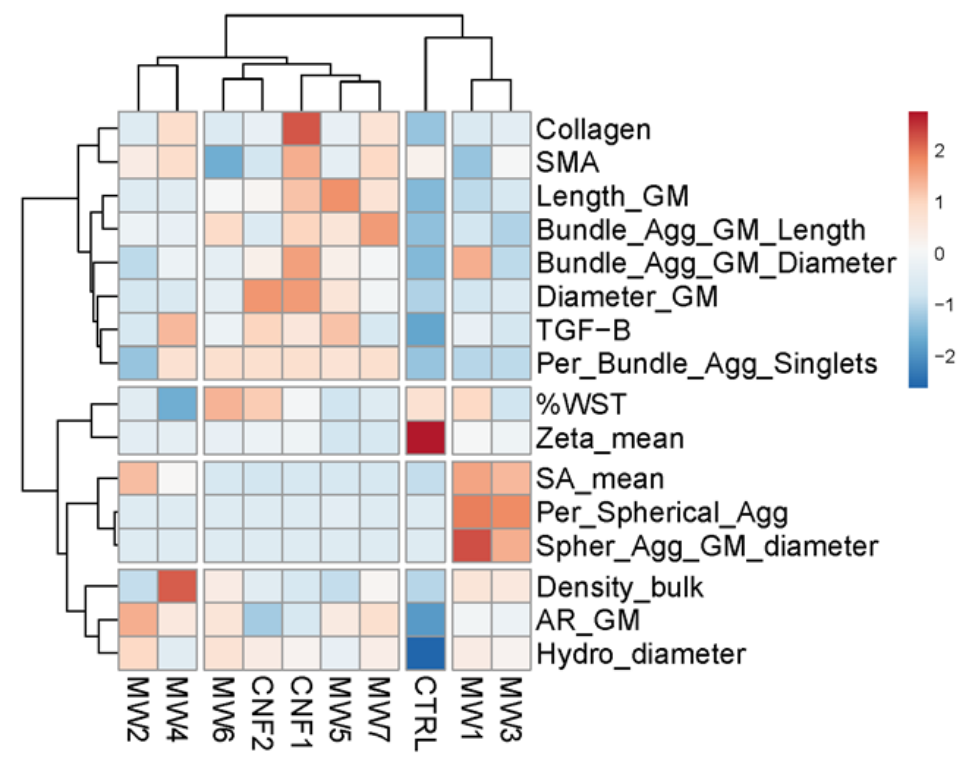

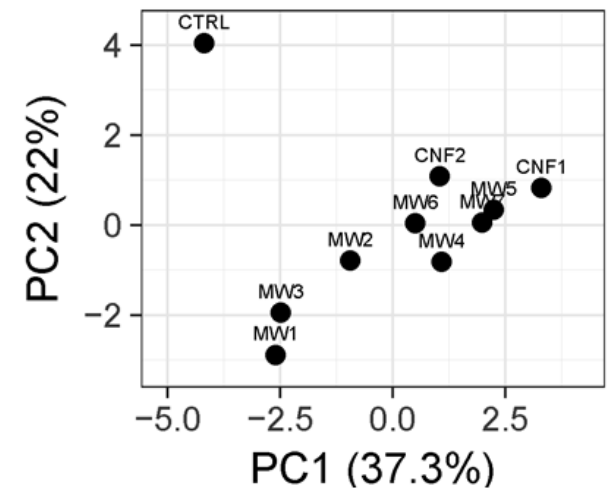

PC1 (37.3\%)
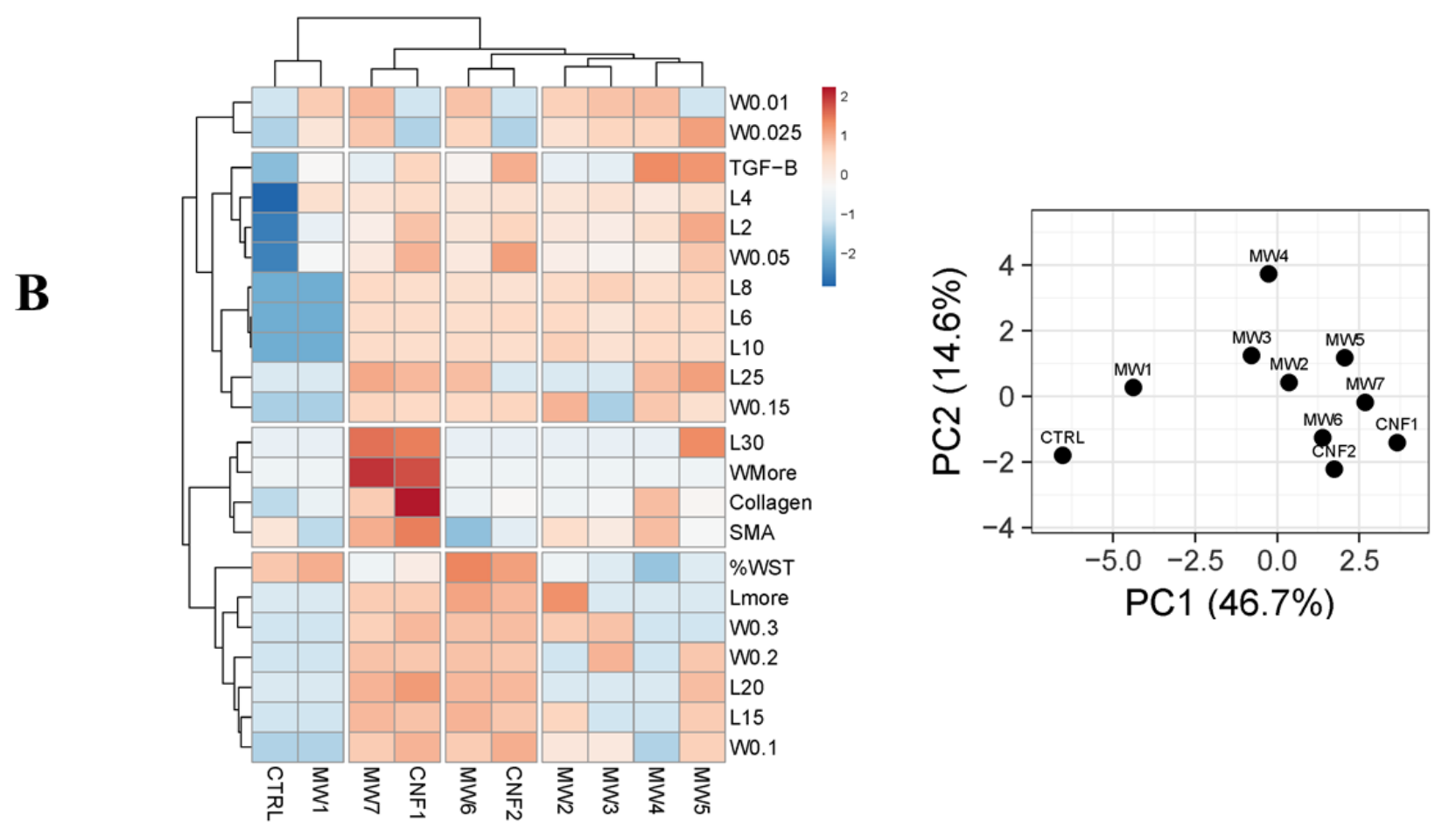

Supplementary Figure 4.11. Hierarchical clustering analysis (HCA) and principal component analysis (PCA) of 'means only' and (A) length-diameter binning (B) with in vitro fibroblast outcomes 


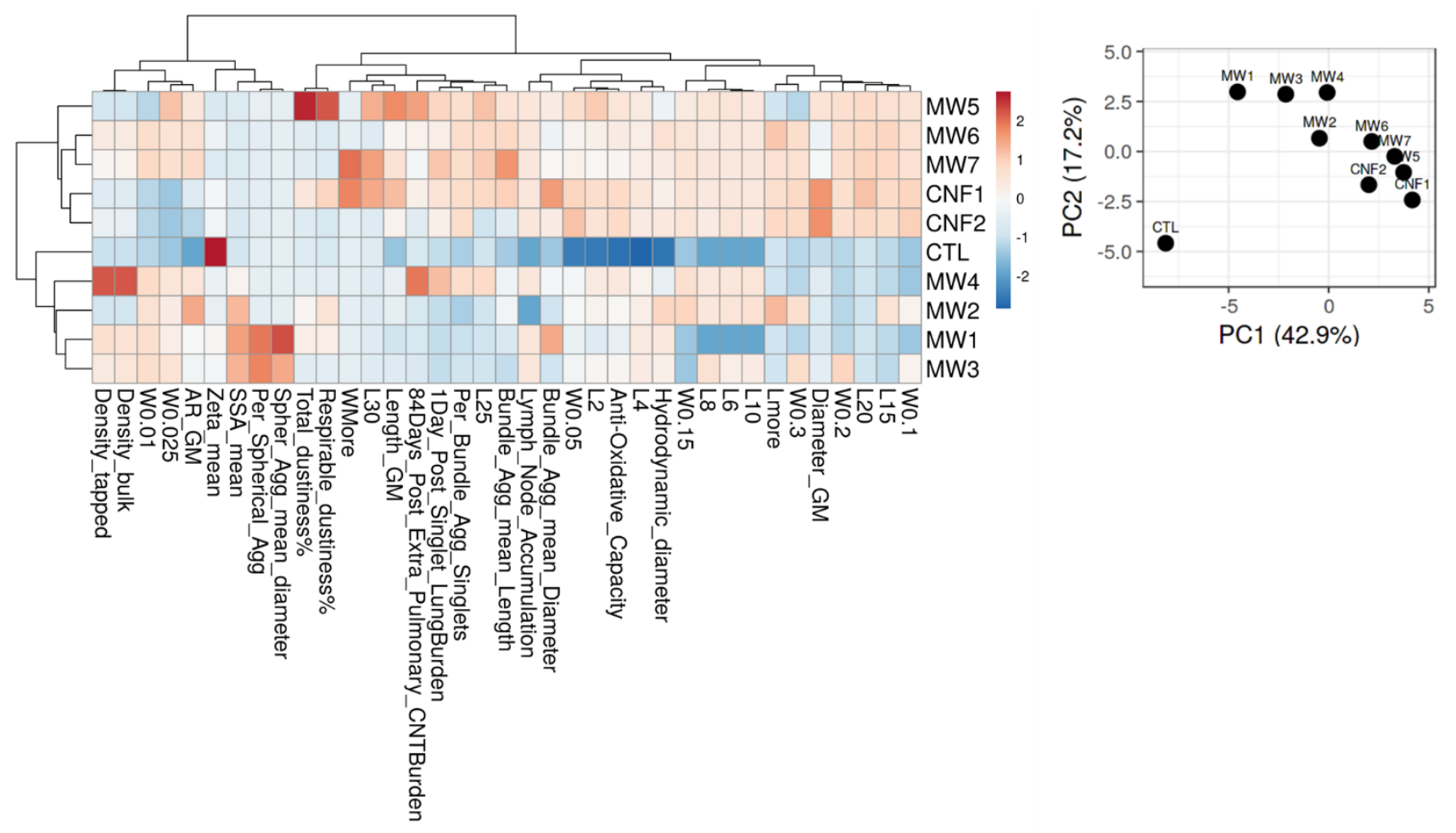

Supplementary Figure 4.12. Hierarchical clustering analysis (HCA) and principal component analysis (PCA) of 'all characterization' and extra-pulmonary translocation outcomes 

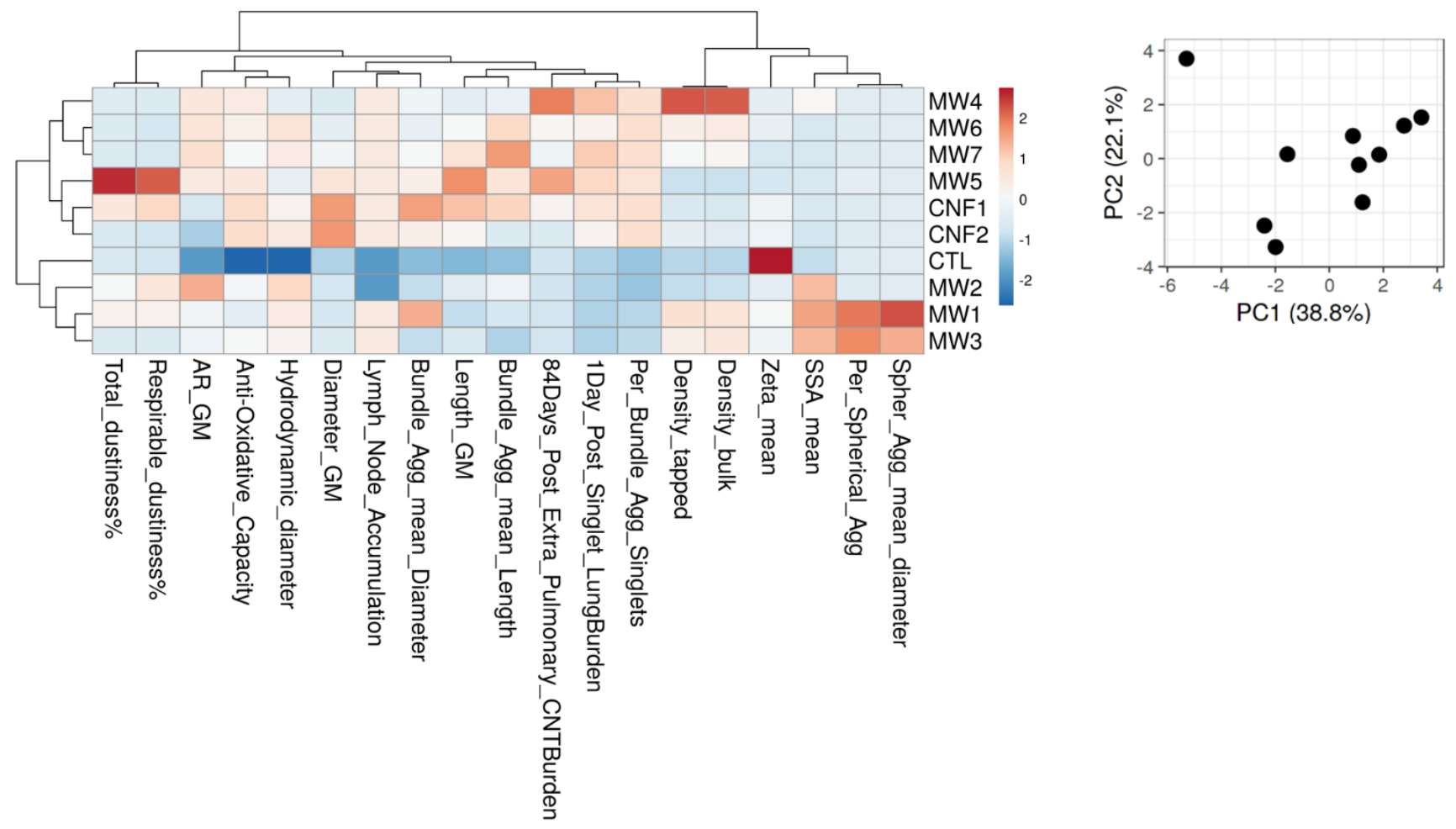

Supplementary Figure 4.13. Hierarchical clustering analysis (HCA) and principal component analysis (PCA) of 'means only' and extra-pulmonary translocation outcomes 


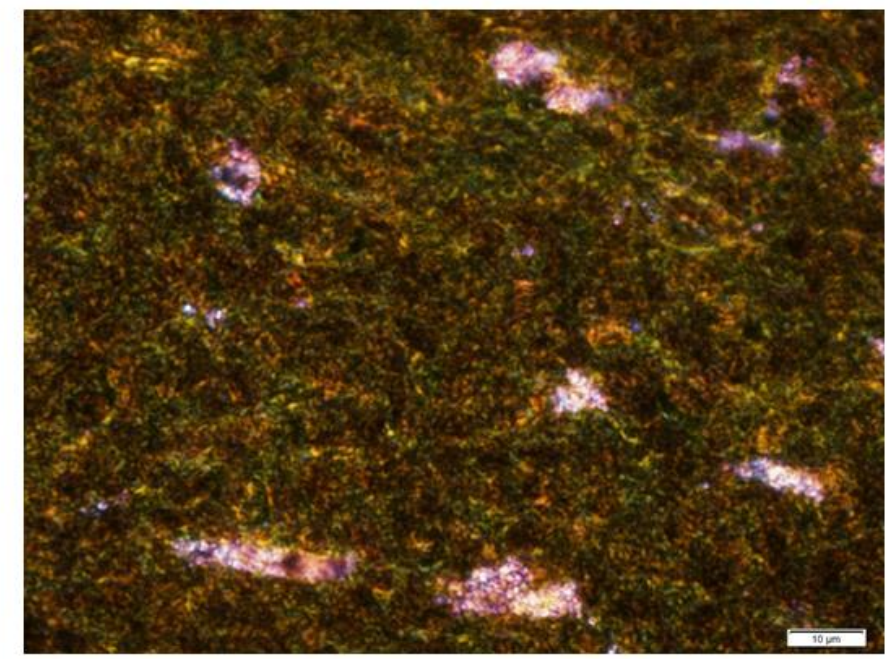

Supplementary Figure 4.14. Previously published image of extra pulmonary translocation and particle accumulation of MW \#3 in the spleen 


\section{CHAPTER 5:}

General Discussion 


\subsection{General Discussion}

The toxicities associated with pulmonary exposure to MWCNTs has been of concern for over a decade driving the demand for the development of exposure modeling systems, expansive toxicity testing, and translational research between in vivo, in vitro, and human studies. This study aimed to expand upon previous toxicity assessments of MWCNT and CNFs by being broadly inclusive of a wide range of particles. Four pillars of toxicity outcomes including genotoxicity, inflammation, histopathology, and extrapulmonary translocation were investigated using a combination of in vivo and in vitro models. Lastly, this study utilized a novel approach to modeling the relationships between these characteristics and toxicity outcomes to identify key drivers.

\subsection{Particles and Characterization}

This study selected nine materials for comparison, including the well-studied Mitsiu-7 MWCNT as a benchmark. Mitsui-7 is a rod-like MWCNT that can be dispersed moderately well while still retaining minimal agglomeration. Averaging around $63 \mathrm{~nm}$ in diameter and $4 \mu \mathrm{m}$ in length, this material generally can be considered to fall in the center of the spectrum of MWCNT and CNF sizes. Four materials of smaller company reported diameter, and four materials of greater company reported diameter were also selected for comparison in this study. Few studies have attempted to compare this large of a group of materials within the same study, and those that do often do not fully represent the full range of particle sizes, thus inadequately addressing important questions. The current study aimed to include this full spectrum of materials of various sizes, agglomeration states, and physicochemical characteristics. However, one noted limitation in this study was the utilization of as-produced materials only, no coatings or surface functionalization were included. While these characteristics are important to safety and are reported to alter to toxicity outcomes, the current study did not include these factors. Other studies 
addressing these factors have been published, including Bishop et al. 2017, and may serve as an additional resource for addressing these concerns [19].

The current study aimed to extensively characterize these materials to maximize the number of variables that may have been considered for their potential influence on toxicity outcomes. One notable advancement in this study compared to numerous previously published studies was the detailed reporting of the distributions and range of particle sizes and histogram presentation. Many studies consider the geometric or arithmetic mean sufficient as the sole reported value, but the conclusions of this study suggest the need for a complete size assessment. While many of the quantified characteristics, such as the antioxidative capacity (FRAS), did not seem to be as potent of a driver as others, these findings are still relevant to the overall conclusions for the future of particle safety-by-design and for future particle toxicity assessments.

\subsection{Translational Considerations}

For the in vivo studies in this project, two key doses were utilized, 4 and $40 \mu \mathrm{g}$ per mouse. The higher of these two doses was selected as previously published studies of the toxicities of MWCNTs have demonstrated that $40 \mu \mathrm{g}$ of MWCNT, particularly Mitsui-7 was necessary to induce significant and notable histopathological changes in the mouse lung, including alveolar fibrosis and inflammation. Due to the previously published implications of this dose in the determination of toxicity outcomes, this dose was also included in the current study design for comparison to the findings of existing studies and to determine the relevance of this dose as a benchmark across all CNT/Fs. While this is a notably higher dose that may not translate efficiently for comparisons to human studies, this historically important dose was necessary for use in this study.

Furthermore, a lesser dose of $4 \mu \mathrm{g}$ per mouse was used due to its relevance of dosimetry to human outcomes. In exposure assessments completed to evaluate human exposures to MWCNT and CNFs in 
U.S. primary and secondary manufacturing facilities, it was reported that workers were exposed to an inhalable elemental carbon mass concentration arithmetic mean of $10.6 \mu \mathrm{g} / \mathrm{m}^{3}$, or $4.07 \mu \mathrm{g} / \mathrm{day}[69,164]$. This dose is equal to $2 \mathrm{ng}$ /day in a mouse of alveolar lung deposition from particle inhalation. Dosimetry calculations would suggest that a $4 \mu \mathrm{g}$ bolus dose would be equal to approximately 7.4 years spent working in a U.S. facility, which at the time of study development, was relevant to the current state and timeline of the industry and was therefore important to include in this study design.

One key goal of the current study was to bridge the translational gap between human exposure assessments and laboratory rodent studies. As the latency of adverse human health outcomes from exposure to $\mathrm{CNT} / \mathrm{F}$ has not been reached, dosimetry studies were designed to monitor human exposure. These studies aim to identify biomarkers of exposure as well as early detection of adverse health outcomes to prevent the onset of toxicity outcomes that may occur. Additionally, these studies assist in the optimization of safety practices and the use of engineering controls and personal protective equipment to ensure safe worker handling.

This study was completed with contributions of several collaborators who completed human exposure assessments, epidemiological studies, and dosimetry assessments to ensure that the rodent model system used in the current study were representative of human exposures. The materials used in this study, with the exception of the reference material, Mitsui-7, were produced in U.S. facilities that were also included in exposure assessments as well as investigations for potential human health effects [68, 69, 79, $92,164]$. As previously mentioned, the doses in this study were selected in consideration of human exposures. Furthermore, the methodology of dispersion of the CNT/F in dispersion media were developed to ensure that the sizes of particles as well as the agglomeration shapes and sizes were similar to those particles observed during human studies. Several toxicity outcomes observed in the mouse model can be also be directly compared to those quantified in human studies such as cytokines, chemokines, and other markers of inflammation and toxicities. 
In vitro modeling systems were also incorporated into the current study in addition to in vivo aspects and human health effects collaborations to challenge the potential of mono-cell culture models and to begin to elucidate the potential mechanisms behind the observed toxicities in vivo. For genotoxicity, inflammation, and histopathology, a relevant cell type was selected and underwent similar toxicity testing.

First, BEAS-2B cells are a human epithelial cell line commonly used in nanoparticle toxicity testing, including genotoxicity assessments. In this study, in vitro testing of genotoxicity was the primary model. In addition to standard testing of cell viability with dose, markers of oxidative stress, $\gamma \mathrm{H} 2 \mathrm{AX}, \mathrm{a}$ marker of double stranded DNA damage, and micronucleus formation, another marker of DNA damage or disruption in cell replication, were assessed. While these assays are broad and cannot fully elucidate the extent of the mechanisms of genotoxicity, alone, they serve as a valuable screening tool for generating comparisons between particles to detect variability in their outcomes. This allows generalizations to be made regarding whether all MWCNT/CNF are equally genotoxic or if some particles act through variable mechanisms leading to genotoxicity outcomes.

Second, for the assessment of inflammation, THP-1 cells, human macrophage cells, were used as a counterpart to the extensive in vivo assays. These cells are also a commonly used model in toxicity assessments as they are relatively easy to culture, can be differentiated to various states of activation, and are capable of phagocytosis. In addition to the dose-response cell viability assay, these cells were also used to assess potential activation of the inflammasome, changes in proteins relevant to cell signaling, cytokine and chemokine release, and tissue repair, as well as changes in phagocytic capacity to determine the potential mechanisms and alterations in cell functions that can occur as a result of particle exposure. These protein changes in particular were useful for direct comparisons between in vivo and in vitro findings as well as an initial screening for changes in basic macrophage activity suggestive of downstream effects of particle exposure. 
Human lung fibroblasts, CRL-1490s, were the third cell model used in this study. While monocell cultures alone cannot be used as a representation of complex multi-cell histopathology changes and cannot be representative of extrapulmonary translocation, these cells were used to attempt to model pulmonary fibrosis and explore potential mechanisms driving the fibrotic response. The in vivo findings in this study revealed the complexities of fibrosis outcomes dependent upon physiological factors such as airway size, region of deposition, and time, that unfortunately, resulted in limited usefulness of fibroblast cultures alone. Two-dimensional monoculture cannot be accurately representative of the regional bronchial or alveolar fibrosis quantified in the in vivo study. Advances in cell culture models such as airliquid interface, lung-on-a-chip, three-dimensional, or co-culture models may be more useful to this application for future studies.

Overall, this study was designed to exemplify human exposures to CNT/F to bridge laboratory and human research together to answer the yet unanswered questions in the field regarding human safety and risk. By including the findings of human exposure assessments in the design of the current study, our model was, to the best of our ability, able to accurately represent human occupational exposures via dose, agglomeration shape and size, and particle dispersion with consideration to assessing endpoints that are translational to human health outcomes.

\subsection{Toxicity Outcomes and Study Findings}

As one key goal of the current study was to identify the relationships between physicochemical characteristics and toxicity outcomes, the first step was to ensure complete and proper characterization for all nine materials. While information on particle sizes and surface chemistry are often reported by the company of production, it is necessary to independently characterize these materials to verify these reports and to expand upon characterization information not reported by manufacturers. In the current study the full panel of characterization included in depth size characterization of length, width, and aspect ratio, 
providing both means and ranges, but a full histogram to identify critical subpopulations of sizes. The agglomeration states of these particles were also defined and measured, and we reported the percentage of tubes present as either spherical or bundled agglomerates and the sizes of those agglomerates. Additionally, the dustiness, density, and anti-oxidative capacity were assessed, and the quantity of potential contaminants including metal catalysts, PAHs, and endotoxin levels were quantified.

Following this characterization, principal component analysis was utilized to first determine if the physicochemical characteristics alone could be used to group similar materials. This initial analysis compared groupings that varied depending on which characteristic data was selected for input. Using the means alone for length, width, and aspect ratio resulted in a different grouping than including bins of particles sizes generated via histogram, which was also somewhat different from the groupings generated when all characterization data was included. L-W-AR and All Characterization analysis grouped MW \#14, MW \#5-7, and CNF \#1-2 together, while including size means alone produced less distinct groupings. Of note, L-W-AR PCA analysis also grouped CNF \#1 as closer to MW \#5-7 while beginning to segregate out CNF \#2. This grouping would later come to relevance with the inclusion of toxicity outcomes in additional analysis. Overall, this grouping was of interest for first determining the variability between the materials used in this study and for laying the groundwork for elucidating which characteristics would play a larger role in subsequent assessments.

Genotoxicity was the first toxicity outcome assessed in the current study. Cell viability, the generation of reactive oxidative species, and the production of cytokines, chemokines, and growth factors were first assessed followed by assessing double stranded DNA breaks and micronuclei formation. These standard assessments provided a useful screening tool for detecting potential DNA damage, though the use of an in vitro model does not provide an accurate assessment of the full carcinogenicity of these materials. This study reported that a significant increase in ROS was found following exposure to high doses of MW \#5-7 and CNF \#1-2, and numerous significant increases in proteins including IL-6, IL-8, 
$\mathrm{IL}-1 \beta$, and other markers of inflammation and cell injury were observed following exposure to both high doses of most materials and low doses some materials. Furthermore, it was reported that all materials were capable of inducing micronuclei formation, and all materials except MW \#2 induced double stranded DNA damage at the higher dose. These findings alone are significant as they indicate that all the materials in the present study can induce genotoxicity regardless of physicochemical characteristics, though the mechanisms and pathways driving this outcome may be variable.

Once completed, these toxicity outcomes were included in the modeling parameters. Hierarchical clustering of the cellular outcomes was combined with the three physicochemical characteristics groups previously modeled. When 'all characteristics' and 'L-W-AR' parameters were included, only two clusters emerged, MW \#1-4 were separated from the rest of the materials. When 'means only' were used, then MW\#4 was switched to the second group containing MW \#5-7 and CNF \#1-2. This is the first suggestion that MW \#4 may be a transitional material between these two groups displaying characteristics and subsequent outcomes that can shift between these two groups, as seen when variable parameters were included in the clustering.

Additionally, this modeling indicated that certain subpopulations of particle sizes, particularly lengths between 15 and $30 \mu \mathrm{m}$ are more distinguishing regarding toxicity outcomes. This is the first notable size population to emerge in relation to toxicity outcomes. Overall, the initial modeling parameters included in part one of the current study have shown that key data points that may not have been previously investigated in regard to drivers of toxicity must be considered and that the average size of a whole population may not be efficient for predicting toxicity outcomes, rather the presence of a particular sub population of particles of a certain size, shape, or characteristic may be more pertinent.

Part two of this study addressed inflammation as a toxicity outcome. First, an in-depth in vivo study investigated outcomes including LDH, inflammatory cell infiltration, and changes in proteins including markers of inflammation, tissue repair, and growth factors at two key doses and four time points. 
Initial observations of these findings suggested that materials of larger diameter were more likely to induce greater and more persistent inflammation. While all materials induced a bolus response and initial acute inflammation, beginning at 7 days post-exposure, several trends began to emerge. Materials of larger diameter and length were associated with more severe and more persistent inflammation. Computer modeling of these outcomes indicated that following a similar trend, MW \#1-4 could be organized into a separate bin from MW \#5-7, and CNF \#1 with CNF \#2 transitioning between the two bins.

In vitro outcomes for inflammation were assessed using human THP-1 macrophages. Almost all materials induced functional changes in the phagocytic capacity of macrophages except for MW \#3. Additionally, all materials resulted in increased caspase-1 activity. All materials induced cell death, significant increases in markers of inflammation and membrane damage, though the dose at which significance was achieved was variable. Similar to in vivo outcomes, materials of larger nominal tube diameter and length were more potently inflammatory than smaller, more agglomerated materials.

Modeling of these outcomes further identified and elucidated these relationships between outcomes and physicochemical characteristics. Several bins of materials were segregated and when all traditional variables as well as length and width bins were considered, the materials were divided into groupings similarly identified with the consideration of genotoxicity outcomes. MW \#1-4 were distinguished from MW \#5-7 and CNF \#1-2. This segregation was confirmation of the frequently observed descriptive trends noting differences between materials and their severity and persistence of inflammation.

The third toxicity outcomes assessed in this project was histopathology outcomes. This assessment was to observe chronic changes in the morphology of the lung as well as additional markers of tissue injury, inflammation, and other changes that may result or be suggestive of long-term functional changes. The outcomes scored in this study include granulomatous inflammation, bronchiolitis obliterans-like changes, alveolar histiocytosis, bronchiolar epithelial hypertrophy, alveolar hypertrophy and hyperplasia, 
and fibrosis, both bronchial and bronchiolar fibrosis. Additional morphometric assessments were made to further quantify and delineate pulmonary fibrosis by region.

Like previously assessed outcomes, these histopathology outcomes were added to the modeling system. When considering the physicochemical characteristics combined with the histopathology outcomes based on their region of effect, the materials can be binned into unique groupings not previously seen in this study, though when all outcomes are considered together, the same trends emerge distinguishing MW \#1-4 from MW \#5-7 and CNF \#1-2, though when considerations were given to region of the lung affected (alveolar or bronchi/bronchiole) there may be some variations in binning.

In addition to these in vivo assessments, human fibroblast cells were employed in vitro assessments to begin to elucidate mechanisms and pathways driving some of these in vivo outcomes. Findings from using a monoculture to represent the entirety of pulmonary fibrosis was limited. As a monoculture is not distinct to the regions of the lung and functionally cannot represent both alveolar and bronchial/bronchiolar regions of the lung, more advanced methods are necessary for a fully representative in vitro model. Incorporation of techniques and culture methods such as air-liquid interface or the "lungon-a-chip" may be necessary for future research.

Translocation was the fourth and final class of toxicity outcomes assessed. The current study was focused on respiratory exposures and respiratory outcomes, though it is well established that particles deposited in the lung may not stay within the lung. Either by diffusion or active transport via macrophages, lymphatics, or systemic circulation, particles may eventually be deposited in other extrapulmonary tissues including the lymph nodes, kidney, liver, spleen, and cardiovascular system. While previous studies of this translocation often suggest limited or very minimal extrapulmonary translocation, clearance from the lung remains a necessary question regarding respiratory toxicology. The current study investigated translocation to the tracheobronchial lymph nodes (TBLN) and the liver. While almost all particles were able to reach the TBLN, the mechanism of transport to the lymph nodes may vary depending on particle 
physicochemical characteristics. Furthermore, translocation to the liver initially suggested three groups of particles, small particles with almost zero to minimal liver deposition, a second group of moderately sized particles with the most liver deposition, and a third group of larger particles that had some, but not extensive liver deposition. These outcomes were further investigated using the established modeling and clustering.

Linear regression modeling suggests that singlet lung burden at one day post-exposure may be useful for predicting liver extra-pulmonary transport at 84 days post-exposure. However, as translocation to other tissues such as the spleen were not assessed, this predictive model for extrapulmonary translocation to all other organ systems is limited. Modeling of translocation outcomes combined with physicochemical characteristics categorized the materials into two bins, similar to the binning previously made using other toxicity outcomes which does not fully match the binning trends made with translocation outcomes alone. This may be due to the computer model placing greater value in the physicochemical characteristics as a means to segregate materials, and prioritizing these differences over the contributions of the translocation outcomes to the overall model

\subsection{Relationships Between Toxicity Outcomes}

The four key toxicity outcomes investigated in this study are not stand-alone considerations. Each of these outcomes are interwoven with one another and influence the severity and progression of adverse health effects (Figure 1). One example is the relationship between inflammation and carcinogenicity. Several biological processes have been identified as being hallmarks of cancer including more recently, inflammation $[174,175]$. Weinberg and Hanahan published an update in 2011 suggesting the role of chronic inflammation as a driver of genotoxicity and cancer [174]. In the current study, several materials, including materials of larger nominal tube dimension were capable of inducing sustained and persistent 
inflammation at 84 days post exposure. This chronic inflammation may therefore be a contributor to the carcinogenicity of MWCNTs.

A second example also related to genotoxicity and cancer. One other hallmark of cancer is the ability of cancer cells to invade other tissues and metastasize $[174,175]$. Activation of TGF $\beta$ through the SMAD signaling pathway is linked to the epithelial-mesenchymal transition of alveolar epithelial cells to a cell phenotype that is capable of tissue invasion and metastasis [187-189]. The current study highlighted increased production of TBF $\beta$ following CNT/F exposure. TGF $\beta$ is also linked to increased collagen-1 deposition and pulmonary fibrosis $[116,186]$. These findings suggest that inflammation, pathology (fibrosis), and genotoxicity are heavily related.

A third example is the relationship between inflammation and extra-pulmonary translocation. Two key mechanisms of translocation have been identified in the current study: macrophage dependent and macrophage independent translocation. Macrophages are an inflammatory cell that plays a role in the clearance of foreign material in the body. In an attempt to clear CNT/F from the lung, macrophages transport particles to other tissues including the lymph nodes and spleen where the particles then are transferred to resident cells of the respective tissues resulting in particle accumulation in extra-pulmonary tissues where they have the potential to induce adverse systemic effects.

\subsection{Contributions of Computational Modeling}

The inclusion of computational modeling was essential to elevate the conclusions of this study and apply quantitative analysis where merely descriptive conclusions would have been previously drawn. These outcomes together conclude that the parameters of nominal diameter, nominal length, and particle agglomeration are the primary divers of toxicity outcomes induced by MWCNT/CNF exposure, and the inclusion of tubes within key size subpopulations can result in more potent genotoxicity, inflammation, histopathology, and extrapulmonary translocation. While not all materials induced the same outcomes, all 
materials induced significant lung injury and possible systemic effects. The physicochemical characteristics of MWCNT/CNF are often interconnected and dependent upon one another. While all characteristics may influence toxicity outcomes, only a few have been identified in this study to be the major contributors impacting the toxicity outcomes. A schematic illustrating this concept can be found in Figure 1.

One key finding in this study was the pertinence of sub populations of particle sizes. The presence of materials greater than $15 \mu \mathrm{m}$ length and $50 \mathrm{~nm}$ in diameter result in more potent and severe effects, even when this population is a minor component of the total particle population. The segregation of these subpopulations using histograms was essential to understanding the outcomes of this study, and due to the lack of this data presented in previously published toxicity assessments, data mining may be a minimally successful endeavor.

Furthermore, particle agglomeration was a notable driver for toxicity outcomes, particularly histopathology outcomes strictly dependent upon regional deposition in the lung. In this study, terminology to describe agglomeration as either "spherical" or "bundled" was created as there is no current standard verbiage to describe differences in CNT agglomeration. For comparisons between multiple studies in the future, shared language and standardized definitions for agglomeration are needed.

\subsection{Impact and Significance on Safety Recommendations}

As the 2020-2024 Report of the Advisory Group to Recommended Priorities for the IARC Monographs has recommended MWCNT as a high priority to be ready for evaluation within five years [37], studies investigating these materials in a wholly inclusive and expansive study is necessary. In this study, it was found that all MWCNT/CNF were genotoxic. While there were differences in the extent and potency between materials, all materials can be assumed to present notable risk that can translate to risk in human exposures. Of note, many of the toxicity outcomes were induced by materials of the low dose, 
which is the dose noted to be most relevant of the REL established by NIOSH. These findings would suggest that the REL may be insufficient as a guideline for protecting facility employees, especially with considering that mouse modeling systems compared to rat models are less sensitive therefore amplifying the importance of adverse effects noted at the lower dose of the current study. Due to the safety factors within the calculations for establishing the REL, often a factor of 10, differences between particles noted int eh current study do not have enough variability between them to warrant specific RELs, one for the broad class as a whole will be sufficient. Additionally, one major limitation to changing the REL is the limit of detection of CNT/F. Currently, the lower limit of detection in air sampling methods is just below the current REL, making a shift to a more restrictive exposure limit unenforceable until advancements in air sampling techniques and technologies advance [57].

Recently, CNTs were added to the "Substitute It Now" (SIN) list in the European union, a list of materials that are designated to be banned once a safer substitute can be identified [196]. However, this designation has been disputed due to the unclear evidence that all CNTs are equally toxic, therefore promoting the desire to ban some, but not all CNTs [197]. Proper understanding of the toxicities of this broad class of materials as a whole and the possibilities of designing safer materials is crucial for the future of the CNT industry and the potential applications for these materials.

\subsection{Future Directions}

The in vitro modeling with BEAS-2B human epithelial cells in the current study were useful as a screening tool for genotoxicity but cannot be used to complete a full assessment of the carcinogenic potential of these materials. Future studies will include an in vivo counterpart in mice to assess tumor promotion, progression, and carcinogenic risk induced by these same materials due to the limited nature of carcinogenicity and genotoxicity assessments using only in vitro modeling. Additionally, using more advanced cell culture systems such as air-liquid interface and other three-dimensional co-culture systems 
may be useful to overcome the limitations of using only human fibroblast cells in monoculture for assessing changes such as pulmonary fibrosis. The application of cutting-edge techniques may improve the relationships elucidated through modeling and may improve the usefulness of in vitro systems in assessing nanotoxicological outcomes.

This study emphasized the importance of modeling methodology and the necessity of inclusion of the most accurate parameters for effective predictions. Future goals and projects will further refine this modeling system and its prediction capabilities. This model may eventually expand to include other carbonaceous nanomaterials of various sizes, dimensions, and characteristics. Additionally, once a functional model has been accepted, software applications for future on-site industry applications and for safety-by-design may become possible. While human epidemiology studies are limited by the latency of time in the industry, efficiently pairing in vitro, in vivo, and in silico modeling in conjunction with human studies will enhance conclusions for future safety and risk assessments. Mechanism discovery as to why some materials induce certain outcomes and the variances in the potency of these outcomes may also be necessary in future studies.

\subsection{Conclusions}

This study was a comprehensive toxicity assessment of MWCNTs and CNFs produced in U.S. facilities. Completion of this study resulted in the creation of an accurate safety profile for seven MWCNTs and two CNFs, and as per the originally stated hypothesis, was able to elucidate that while all materials in this family can be toxic, the toxicity outcomes induced are dependent upon physicochemical characteristics, importantly materials comprised of singlets and well dispersed fibers with notable subpopulations of tubes with larger nominal diameters and lengths have a greater likelihood of inducing greater and more persistent inflammation, histopathology changes in the bronchi and bronchioles including pulmonary fibrosis. Materials with subpopulations of lengths within a moderate length and 
diameter have an increased likelihood of extra-pulmonary translocation to tissues such as the liver, though size may not be a critical determinant of the likelihood of a particle to induce genotoxicity. Overall, identification of these critical parameters is important to understanding the risks of human adverse health effects, for the future of industry safety practices, and the design of new, safer nanomaterials. 


\section{CHAPTER 5 FIGURES}

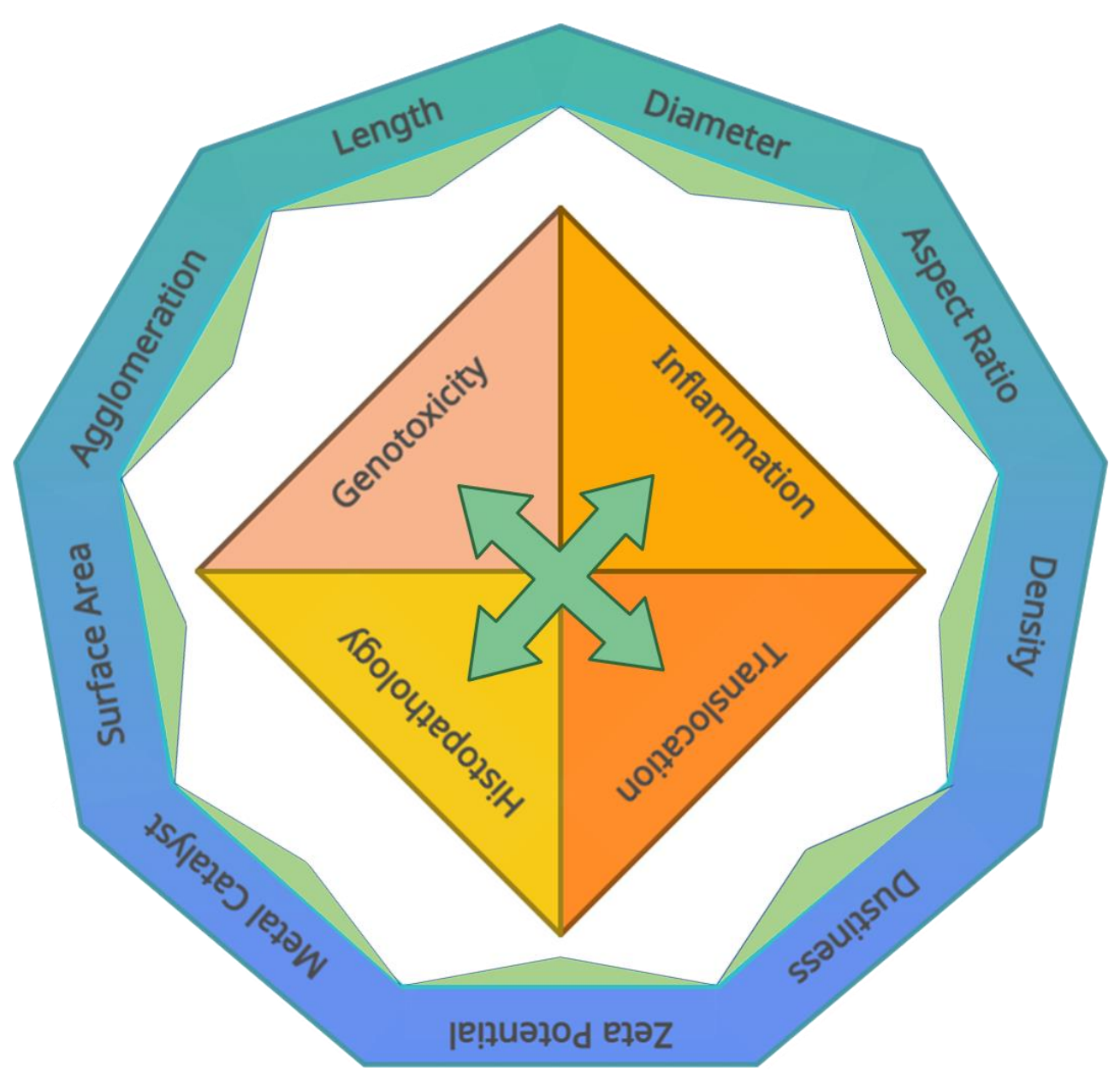

Figure 5.1. Schematic of the physicochemical characteristics, the four major toxicity outcomes, and the relationships between them. 


\section{REFERENCES}

1. De Volder, M.F., et al., Carbon nanotubes: present and future commercial applications. Science, 2013. 339(6119): p. 535-9.

2. Geng, Y., et al., Effects of surfactant treatment on mechanical and electrical properties of CNT/epoxy nanocomposites. Composites, 2008. 39: p. 1876-1883.

3. Kim, S.W., et al., Surface modifications for the effective dispersion of carbon nanotubes in solvents and polymers. Carbon, 2012. 50(1): p. 3-33.

4. Pras, M., et al., Key Role of the Dispersion of Carbon Nanotubes (CNTs) within Epoxy Networks on their Ability to Release. Polymers (Basel), 2020. 12(11).

5. Stanton, M.F., Fiber Carcinogenesis: Is Asbestos the Only Hazard? J Natl Cancer Inst, 1974. 52(3): p. 6334.

6. Donaldson, K., et al., Asbestos, carbon nanotubes and the pleural mesothelium: a review of the hypothesis regarding the role of long fibre retention in the parietal pleura, inflammation and mesothelioma. Part Fibre Toxicol, 2010. 7: p. 5.

7. Donaldson, K., et al., Pulmonary toxicity of carbon nanotubes and asbestos - similarities and differences. Adv Drug Deliv Rev, 2013. 65(15): p. 2078-86.

8. Boyles, M.S., et al., Multi-walled carbon nanotube induced frustrated phagocytosis, cytotoxicity and proinflammatory conditions in macrophages are length dependent and greater than that of asbestos. Toxicol In Vitro, 2015. 29(7): p. 1513-28.

9. Mercer, R.R., et al., Pulmonary fibrotic response to aspiration of multi-walled carbon nanotubes. Part Fibre Toxicol, 2011. 8: p. 21.

10. Mercer, R.R., et al., Distribution and fibrotic response following inhalation exposure to multi-walled carbon nanotubes. Part Fibre Toxicol, 2013. 10: p. 33.

11. Knudsen, K.B., et al., Physicochemical predictors of Multi-Walled Carbon Nanotube-induced pulmonary histopathology and toxicity one year after pulmonary deposition of 11 different Multi-Walled Carbon Nanotubes in mice. Basic Clin Pharmacol Toxicol, 2019. 124(2): p. 211-227.

12. Poulsen, S.S., et al., Multi-walled carbon nanotube physicochemical properties predict pulmonary inflammation and genotoxicity. Nanotoxicology, 2016. 10(9): p. 1263-75.

13. Fenoglio, I., et al., Thickness of multiwalled carbon nanotubes affects their lung toxicity. Chem Res Toxicol, 2012. 25(1): p. 74-82.

14. Nagai, H., et al., Diameter and rigidity of multiwalled carbon nanotubes are critical factors in mesothelial injury and carcinogenesis. Proc Natl Acad Sci U S A, 2011. 108(49): p. E1330-8.

15. Oberdorster, G., et al., Inhalation Exposure to Carbon Nanotubes (CNT) and Carbon Nanofibers (CNF): Methodology and Dosimetry. J Toxicol Environ Health B Crit Rev, 2015. 18(3-4): p. 121-212.

16. Su, W.C. and Y.S. Cheng, Carbon nanotubes size classification, characterization and nasal airway deposition. Inhal Toxicol, 2014. 26(14): p. 843-52.

17. Sturm, R., Carbon Nanotubes in the Human Respiratory Tract-Clearance Modeling. Ann Work Expo Health, 2017. 61(2): p. 226-236.

18. Sturm, R., A stochastic model of carbon nanotube deposition in the airways and alveoli of the human respiratory tract. Inhal Toxicol, 2016. 28(2): p. 49-60.

19. Bishop, L., et al., In Vivo Toxicity Assessment of Occupational Components of the Carbon Nanotube Life Cycle To Provide Context to Potential Health Effects. ACS Nano, 2017. 11(9): p. 8849-8863.

20. Evans, D.E., et al., Dustiness of fine and nanoscale powders. Ann Occup Hyg, 2013. 57(2): p. 261-77.

21. Yu, M., Fundamental Mechanical Properties of Carbon Nanotubes: Current Understanding and the Related Experimental Studies. Journal of Engineering Materials and Technology, 2004. 126: p. 271-278.

22. Birch, M.E., et al., Properties that influence the specific surface areas of carbon nanotubes and nanofibers. Ann Occup Hyg, 2013. 57(9): p. 1148-66.

23. Long, J., et al., The adverse vascular effects of multi-walled carbon nanotubes (MWCNTs) to human vein endothelial cells (HUVECS) in vitro: role of length of MWCNTs. J Nanobiotechnology, 2017. 15(1): p. 80. 
24. Donaldson, K., et al., Carbon nanotubes: a review of their properties in relation to pulmonary toxicology and workplace safety. Toxicol Sci, 2006. 92(1): p. 5-22.

25. Chen, B., et al., In Vitro Evaluation of Cytotoxicity and Oxidative Stress Induced by Multiwalled Carbon Nanotubes in Murine RAW 264.7 Macrophages and Human A549 Lung Cells. Biomedical and Environmental Sciences, 2011. 24(6): p. 593-601.

26. Kagan, V.E., et al., Direct and indirect effects of single walled carbon nanotubes on RAW 264.7 macrophages: role of iron. Toxicol Lett, 2006. 165(1): p. 88-100.

27. Inoue, K.-i., et al., Repeated pulmonary exposure to single-walled carbon nanotubes exacerbates allergic inflammation of the airway: Possible role of oxidative stress. Free Radical Biology and Medicine, 2010. 48(7): p. 924-934.

28. Pacurari, M., et al., Raw single-wall carbon nanotubes induce oxidative stress and activate MAPKs, AP-1, NF-kappaB, and Akt in normal and malignant human mesothelial cells. Environ Health Perspect, 2008. 116(9): p. 1211-7.

29. Lee, D.-K., et al., Potential Role of Soluble Metal Impurities in the Acute Lung Inflammogenicity of MultiWalled Carbon Nanotubes. Nanomaterials (Basel, Switzerland), 2020. 10(2): p. 379.

30. Shvedova, A.A., et al., Exposure to carbon nanotube material: assessment of nanotube cytotoxicity using human keratinocyte cells. J Toxicol Environ Health A, 2003. 66(20): p. 1909-26.

31. Fenoglio, I., et al., Structural defects play a major role in the acute lung toxicity of multiwall carbon nanotubes: physicochemical aspects. Chem Res Toxicol, 2008. 21(9): p. 1690-7.

32. Fenoglio, I., et al., Reactivity of carbon nanotubes: free radical generation or scavenging activity? Free Radic Biol Med, 2006. 40(7): p. 1227-33.

33. Hussain, S., et al., Multiwalled Carbon Nanotube Functionalization with High Molecular Weight Hyaluronan Significantly Reduces Pulmonary Injury. ACS Nano, 2016. 10(8): p. 7675-88.

34. Landry, M., et al., Early signs of multi-walled carbon nanotbues degradation in macrophages, via an intracellular $\mathrm{pH}$-dependent biological mechanism; importance of length and functionalization. Part Fibre Toxicol, 2016. 13(1): p. 61.

35. Wang, X., et al., Dispersal state of multiwalled carbon nanotubes elicits profibrogenic cellular responses that correlate with fibrogenesis biomarkers and fibrosis in the murine lung. ACS Nano, 2011. 5(12): p. 977287.

36. Taylor-Just, A.J., et al., The pulmonary toxicity of carboxylated or aminated multi-walled carbon nanotubes in mice is determined by the prior purification method. Part Fibre Toxicol, 2020. 17(1): p. 60.

37. IARC. Report of the Advisory Group to Recommend Priorities for the IARC Monographs during 2020-2024. in IARC Monographs on the Identification of Carcinogenic Hazards to Humans. 2020. International Agency for Research on Cancer.

38. Takagi, A., et al., Induction of mesothelioma in p53+/- mouse by intraperitoneal application of multi-wall carbon nanotube. J Toxicol Sci, 2008. 33(1): p. 105-16.

39. Takagi, A., et al., Dose-dependent mesothelioma induction by intraperitoneal administration of multi-wall carbon nanotubes in p53 heterozygous mice. Cancer Sci, 2012. 103(8): p. 1440-4.

40. Sakamoto, Y., et al., Induction of mesothelioma by a single intrascrotal administration of multi-wall carbon nanotube in intact male Fischer 344 rats. J Toxicol Sci, 2009. 34(1): p. 65-76.

41. Miller, B.G., et al., Influence of fibre length, dissolution and biopersistence on the production of mesothelioma in the rat peritoneal cavity. Ann Occup Hyg, 1999. 43(3): p. 155-66.

42. Sargent, L.M., et al., Promotion of lung adenocarcinoma following inhalation exposure to multi-walled carbon nanotubes. Part Fibre Toxicol, 2014. 11: p. 3.

43. Kasai, T., et al., Thirteen-week study of toxicity of fiber-like multi-walled carbon nanotubes with wholebody inhalation exposure in rats. Nanotoxicology, 2015. 9(4): p. 413-22.

44. Grosse, Y., et al., Carcinogenicity of fluoro-edenite, silicon carbide fibres and whiskers, and carbon nanotubes. Lancet Oncol, 2014. 15(13): p. 1427-1428.

45. Sargent, L.M., et al., Single-walled carbon nanotube-induced mitotic disruption. Mutat Res, 2012. 745(12): p. 28-37. 
46. Siegrist, K.J., et al., Genotoxicity of multi-walled carbon nanotubes at occupationally relevant doses. Part Fibre Toxicol, 2014. 11: p. 6.

47. Siegrist, K.J., et al., Mitsui-7, heat-treated, and nitrogen-doped multi-walled carbon nanotubes elicit genotoxicity in human lung epithelial cells. Part Fibre Toxicol, 2019. 16(1): p. 36.

48. Muller, J., et al., Clastogenic and aneugenic effects of multi-wall carbon nanotubes in epithelial cells. Carcinogenesis, 2008. 29(2): p. 427-33.

49. Muller, J., et al., Respiratory toxicity of multi-wall carbon nanotubes. Toxicol Appl Pharmacol, 2005. 207(3): p. 221-31.

50. Ma-Hock, L., et al., Inhalation toxicity of multiwall carbon nanotubes in rats exposed for 3 months. Toxicol Sci, 2009. 112(2): p. 468-81.

51. Porter, D.W., et al., Mouse pulmonary dose- and time course-responses induced by exposure to multiwalled carbon nanotubes. Toxicology, 2010. 269(2-3): p. 136-47.

52. Sweeney, S., et al., Multi-walled carbon nanotube length as a critical determinant of bioreactivity with primary human pulmonary alveolar cells. Carbon N Y, 2014. 78: p. 26-37.

53. Delorme, M.P., et al., Ninety-day inhalation toxicity study with a vapor grown carbon nanofiber in rats. Toxicol Sci, 2012. 128(2): p. 449-60.

54. Hamilton, R.F., Jr., et al., Length, but Not Reactive Edges, of Cup-stack MWCNT Is Responsible for Toxicity and Acute Lung Inflammation. Toxicol Pathol, 2018. 46(1): p. 62-74.

55. Renne, R., et al., Proliferative and nonproliferative lesions of the rat and mouse respiratory tract. Toxicol Pathol, 2009. 37(7 Suppl): p. 5s-73s.

56. Duke, K.S., et al., STAT1-dependent and-independent pulmonary allergic and fibrogenic responses in mice after exposure to tangled versus rod-like multi-walled carbon nanotubes. Part Fibre Toxicol, 2017. 14(1): p. 26.

57. NIOSH, Current Intelligence Bulletin 65: Occupational Exposure to Carbon Nanotubes and Nanofibers. National Institute for Occupational Safety and Health 2013.

58. Mercer, R.R., et al., Distribution and persistence of pleural penetrations by multi-walled carbon nanotubes. Part Fibre Toxicol, 2010. 7: p. 28.

59. Czarny, B., et al., Carbon nanotube translocation to distant organs after pulmonary exposure: insights from in situ (14)C-radiolabeling and tissue radioimaging. ACS Nano, 2014. 8(6): p. 5715-24.

60. Aiso, S., et al., Translocation of intratracheally instilled multiwall carbon nanotubes to lung-associated lymph nodes in rats. Ind Health, 2011. 49(2): p. 215-20.

61. Pauluhn, J., Multi-walled carbon nanotubes (Baytubes): approach for derivation of occupational exposure limit. Regul Toxicol Pharmacol, 2010. 57(1): p. 78-89.

62. Brook, R.D., et al., Particulate matter air pollution and cardiovascular disease: An update to the scientific statement from the American Heart Association. Circulation, 2010. 121(21): p. 2331-78.

63. Mostovenko, E., et al., Nanoparticle exposure driven circulating bioactive peptidome causes systemic inflammation and vascular dysfunction. Part Fibre Toxicol, 2019. 16(1): p. 20.

64. Mercer, R.R., et al., Extrapulmonary transport of MWCNT following inhalation exposure. Part Fibre Toxicol, 2013. 10: p. 38.

65. Ryman-Rasmussen, J.P., et al., Inhaled carbon nanotubes reach the subpleural tissue in mice. Nat Nanotechnol, 2009. 4(11): p. 747-51.

66. Reddy, A.R., et al., Translocation and extra pulmonary toxicities of multi wall carbon nanotubes in rats. Toxicol Mech Methods, 2010. 20(5): p. 267-72.

67. Ingle, T., et al., Raman spectroscopy analysis and mapping the biodistribution of inhaled carbon nanotubes in the lungs and blood of mice. J Appl Toxicol, 2013. 33(10): p. 1044-52.

68. Dahm, M.M., et al., Exposure assessments for a cross-sectional epidemiologic study of US carbon nanotube and nanofiber workers. Int J Hyg Environ Health, 2018. 221(3): p. 429-440.

69. Dahm, M.M., et al., Carbon Nanotube and Nanofiber Exposure Assessments: An Analysis of 14 Site Visits. Ann Occup Hyg, 2015. 59(6): p. 705-23. 
70. Liao, H.Y., et al., Six-month follow-up study of health markers of nanomaterials among workers handling engineered nanomaterials. Nanotoxicology, 2014. 8 Suppl 1: p. 100-10.

71. Liou, S.-H., et al., Epidemiological study of health hazards among workers handling engineered nanomaterials. Journal of Nanoparticle Research, 2012. 14(8).

72. Lee, J.H., et al., Three-Day Continuous Exposure Monitoring of CNT Manufacturing Workplaces. Biomed Res Int, 2015. 2015: p. 237140.

73. Fatkhutdinova, L.M., et al., Fibrosis biomarkers in workers exposed to MWCNTs. Toxicol Appl Pharmacol, 2016. 299: p. 125-31.

74. Shvedova, A.A., et al., Integrated Analysis of Dysregulated ncRNA and mRNA Expression Profiles in Humans Exposed to Carbon Nanotubes. PLoS One, 2016. 11(3): p. e0150628.

75. Ghosh, M., et al., Changes in DNA methylation induced by multi-walled carbon nanotube exposure in the workplace. Nanotoxicology, 2017. 11(9-10): p. 1195-1210.

76. Kuijpers, E., et al., Cardiovascular effects among workers exposed to multiwalled carbon nanotubes. Occup Environ Med, 2018. 75(5): p. 351-358.

77. Vlaanderen, J., et al., A cross-sectional study of changes in markers of immunological effects and lung health due to exposure to multi-walled carbon nanotubes. Nanotoxicology, 2017. 11(3): p. 395-404.

78. Beard, J.D., et al., Carbon nanotube and nanofiber exposure and sputum and blood biomarkers of early effect among U.S. workers. Environ Int, 2018. 116: p. 214-228.

79. Schubauer-Berigan, M.K., et al., Association of pulmonary, cardiovascular, and hematologic metrics with carbon nanotube and nanofiber exposure among U.S. workers: a cross-sectional study. Part Fibre Toxicol, 2018. 15(1): p. 22.

80. Bannuscher, A., et al., A multi-omics approach reveals mechanisms of nanomaterial toxicity and structureactivity relationships in alveolar macrophages. Nanotoxicology, 2020. 14(2): p. 181-195.

81. Warheit, D.B., et al., Comparative pulmonary toxicity assessment of single-wall carbon nanotubes in rats. Toxicol Sci, 2004. 77(1): p. 117-25.

82. Lam, C.W., et al., Pulmonary toxicity of single-wall carbon nanotubes in mice 7 and 90 days after intratracheal instillation. Toxicol Sci, 2004. 77(1): p. 126-34.

83. Erdely, A., et al., Identification of systemic markers from a pulmonary carbon nanotube exposure. J Occup Environ Med, 2011. 53(6 Suppl): p. S80-6.

84. Kasai, T., et al., Development of a new multi-walled carbon nanotube (MWCNT) aerosol generation and exposure system and confirmation of suitability for conducting a single-exposure inhalation study of MWCNT in rats. Nanotoxicology, 2014. 8(2): p. 169-78.

85. Mitchell, L.A., et al., Pulmonary and systemic immune response to inhaled multiwalled carbon nanotubes. Toxicol Sci, 2007. 100(1): p. 203-14.

86. Pauluhn, J., Subchronic 13-week inhalation exposure of rats to multiwalled carbon nanotubes: toxic effects are determined by density of agglomerate structures, not fibrillar structures. Toxicol Sci, 2010. 113(1): p. 226-42.

87. Erdely, A., et al., Cross-talk between lung and systemic circulation during carbon nanotube respiratory exposure. Potential biomarkers. Nano Lett, 2009. 9(1): p. 36-43.

88. Aragon, M.J., et al., Serum-borne bioactivity caused by pulmonary multiwalled carbon nanotubes induces neuroinflammation via blood-brain barrier impairment. Proc Natl Acad Sci U S A, 2017. 114(10): p. E1968E1976.

89. Schubauer-Berigan, M.K., et al., Association of occupational exposures with ex vivo functional immune response in workers handling carbon nanotubes and nanofibers. Nanotoxicology, 2020. 14(3): p. 404-419.

90. Kuijpers, E., et al., Occupational Exposure to Multi-Walled Carbon Nanotubes During Commercial Production Synthesis and Handling. Ann Occup Hyg, 2016. 60(3): p. 305-17.

91. Canu, G.I., et al., State of knowledge on the occupational exposure to carbon nanotubes. Int J Hyg Environ Health, 2020. 225: p. 113472.

92. Dahm, M.M., et al., Occupational exposure assessment in carbon nanotube and nanofiber primary and secondary manufacturers. Ann Occup Hyg, 2012. 56(5): p. 542-56. 
93. Hamilton, R.F., Jr., et al., Effect of MWCNT size, carboxylation, and purification on in vitro and in vivo toxicity, inflammation and lung pathology. Part Fibre Toxicol, 2013. 10(1): p. 57.

94. Jackson, P., et al., Characterization of genotoxic response to 15 multiwalled carbon nanotubes with variable physicochemical properties including surface functionalizations in the FE1-Muta(TM) mouse lung epithelial cell line. Environ Mol Mutagen, 2015. 56(2): p. 183-203.

95. Li, R., et al., Surface charge and cellular processing of covalently functionalized multiwall carbon nanotubes determine pulmonary toxicity. ACS Nano, 2013. 7(3): p. 2352-68.

96. Murphy, F.A., et al., Length-dependent retention of carbon nanotubes in the pleural space of mice initiates sustained inflammation and progressive fibrosis on the parietal pleura. Am J Pathol, 2011. 178(6): p. 2587600.

97. Murphy, F.A., et al., The mechanism of pleural inflammation by long carbon nanotubes: interaction of long fibres with macrophages stimulates them to amplify pro-inflammatory responses in mesothelial cells. Part Fibre Toxicol, 2012. 9: p. 8.

98. Sager, T.M., et al., Effect of multi-walled carbon nanotube surface modification on bioactivity in the C57BL/6 mouse model. Nanotoxicology, 2014. 8(3): p. 317-27.

99. Ali-Boucetta, H., et al., Cellular uptake and cytotoxic impact of chemically functionalized and polymercoated carbon nanotubes. Small, 2011. 7(22): p. 3230-8.

100. Frohlich, E., et al., Combination of small size and carboxyl functionalisation causes cytotoxicity of short carbon nanotubes. Nanotoxicology, 2013. 7(7): p. 1211-24.

101. Gernand, J.M. and E.A. Casman, A meta-analysis of carbon nanotube pulmonary toxicity studies--how physical dimensions and impurities affect the toxicity of carbon nanotubes. Risk Anal, 2014. 34(3): p. 58397.

102. WHO, The WHO/EURO man-made mineral fiber reference scheme. By the WHO/EURO Technical Committee for Monitoring and Evaluating MMMF, in Scand J Work Environ Health. 1985, World Health Organization. p. 123-9.

103. Bertrand, R. and H. Pezerat, Fibrous glass: carcinogenicity and dimensional characteristics. IARC Sci Publ, 1980(30): p. 901-11.

104. Brown, R.C., et al., The effect of fibre size on the in vitro biological activity of three types of amphibole asbestos. Int J Cancer, 1978. 22(6): p. 721-7.

105. Davis, J.M., A review of experimental evidence for the carcinogenicity of man-made vitreous fibers. Scand J Work Environ Health, 1986. 12 Suppl 1: p. 12-7.

106. Dogra, S. and K. Donaldson, Effect of long and short fibre amosite asbestos on in vitro TNF production by rat alveolar macrophages: the modifying effect of lipopolysaccharide. Ind Health, 1995. 33(3): p. 131-41.

107. Hart, G.A., L.M. Kathman, and T.W. Hesterberg, In vitro cytotoxicity of asbestos and man-made vitreous fibers: roles of fiber length, diameter and composition. Carcinogenesis, 1994. 15(5): p. 971-7.

108. Hesterberg, T.W. and J.C. Barrett, Dependence of asbestos- and mineral dust-induced transformation of mammalian cells in culture on fiber dimension. Cancer Res, 1984. 44(5): p. 2170-80.

109. Poland, C.A., et al., Length-dependent pathogenic effects of nickel nanowires in the lungs and the peritoneal cavity. Nanotoxicology, 2012. 6: p. 899-911.

110. Schinwald, A., et al., The threshold length for fiber-induced acute pleural inflammation: shedding light on the early events in asbestos-induced mesothelioma. Toxicol Sci, 2012. 128(2): p. 461-70.

111. Stanton, M.F., et al., Relation of particle dimension to carcinogenicity in amphibole asbestoses and other fibrous minerals. J Natl Cancer Inst, 1981. 67(5): p. 965-75.

112. Stanton, M.F., et al., Carcinogenicity of fibrous glass: pleural response in the rat in relation to fiber dimension. J Natl Cancer Inst, 1977. 58(3): p. 587-603.

113. Boulanger, G., et al., Quantification of short and long asbestos fibers to assess asbestos exposure: a review of fiber size toxicity. Environ Health, 2014. 13: p. 59.

114. Kane, A.B., R.H. Hurt, and H. Gao, The asbestos-carbon nanotube analogy: An update. Toxicol Appl Pharmacol, 2018. 361: p. 68-80. 
115. IARC. Some Nanomaterials and Some Fibres. in IARC Monographs of the Evaluation of Carcinogenic Risks to Humans. 2014. International Agency for Research on Cancer.

116. Manke, A., et al., Effect of fiber length on carbon nanotube-induced fibrogenesis. Int J Mol Sci, 2014. 15(5): p. 7444-61.

117. Muhlfeld, C., et al., Differential effects of long and short carbon nanotubes on the gas-exchange region of the mouse lung. Nanotoxicology, 2012. 6: p. 867-79.

118. Murphy, F.A., et al., Length-dependent pleural inflammation and parietal pleural responses after deposition of carbon nanotubes in the pulmonary airspaces of mice. Nanotoxicology, 2013. 7(6): p. 115767.

119. Osmond-McLeod, M.J., et al., Durability and inflammogenic impact of carbon nanotubes compared with asbestos fibres. Part Fibre Toxicol, 2011. 8: p. 15.

120. Poland, C.A., et al., Carbon nanotubes introduced into the abdominal cavity of mice show asbestos-like pathogenicity in a pilot study. Nat Nanotechnol, 2008. 3(7): p. 423-8.

121. Poulsen, S.S., et al., Multi-walled carbon nanotube-physicochemical properties predict the systemic acute phase response following pulmonary exposure in mice. PLoS One, 2017. 12(4): p. e0174167.

122. Allegri, M., et al., Toxicity determinants of multi-walled carbon nanotubes: The relationship between functionalization and agglomeration. Toxicol Rep, 2016. 3: p. 230-243.

123. Catalan, J., et al., In vitro and in vivo genotoxic effects of straight versus tangled multi-walled carbon nanotubes. Nanotoxicology, 2016. 10(6): p. 794-806.

124. Duke, K.S., et al., Role of $p 53$ in the chronic pulmonary immune response to tangled or rod-like multi-walled carbon nanotubes. Nanotoxicology, 2018. 12(9): p. 975-991.

125. Donaldson, K. and V. Stone, Current hypotheses on the mechanisms of toxicity of ultrafine particles. Ann Ist Super Sanita, 2003. 39(3): p. 405-10.

126. Sager, T.M. and V. Castranova, Surface area of particle administered versus mass in determining the pulmonary toxicity of ultrafine and fine carbon black: comparison to ultrafine titanium dioxide. Part Fibre Toxicol, 2009. 6: p. 15.

127. Sager, T.M., C. Kommineni, and V. Castranova, Pulmonary response to intratracheal instillation of ultrafine versus fine titanium dioxide: role of particle surface area. Part Fibre Toxicol, 2008. 5: p. 17.

128. Schmid, O. and T. Stoeger, Surface area is the biologically most effective dose metric for acute nanoparticle toxicity in the lung. Journal of Aerosol Science, 2016. 99: p. 133-143.

129. Stoeger, T., et al., Instillation of six different ultrafine carbon particles indicates a surface area threshold dose for acute lung inflammation in mice. Environ Health Perspect, 2006. 114(3): p. 328-33.

130. Decker, J.E., et al., Sample preparation protocols for realization of reproducible characterization of singlewall carbon nanotubes. Metrologia, 2009. 46(6): p. 682-692.

131. Ye, Y., et al., Hydrogen adsorption and cohesive energy of single-walled carbon nanotubes. Applied Physics Letters, 1999. 74(16): p. 2307-2309.

132. Manshian, B.B., et al., Single-walled carbon nanotubes: differential genotoxic potential associated with physico-chemical properties. Nanotoxicology, 2013. 7(2): p. 144-56.

133. Dahm, M.M., et al., Evaluation of total and inhalable samplers for the collection of carbon nanotube and carbon nanofiber aerosols. Aerosol Science and Technology, 2019. 53(8): p. 958-970.

134. Drew, N.M., et al., A quantitative framework to group nanoscale and microscale particles by hazard potency to derive occupational exposure limits: Proof of concept evaluation. Regul Toxicol Pharmacol, 2017. 89: p. 253-267.

135. Bahl, A., et al., Recursive feature elimination in random forest classification supports nanomaterial grouping. Nanolmpact, 2019. 15.

136. Liu, X., et al., Bioavailability of Nickel in Single-Wall Carbon Nanotubes. 2007. 19(19): p. 2790-2796.

137. Lee, V., et al., Amphiphilic polymer-coated CdSe/ZnS quantum dots induce pro-inflammatory cytokine expression in mouse lung epithelial cells and macrophages. Nanotoxicology, 2015. 9(3): p. 336-43. 
138. Chalbot, M.G., et al., Synergistic effects of engineered nanoparticles and organics released from laser printers using nano-enabled toners: potential health implications from exposures to the emitted organic aerosol. Environ Sci Nano, 2017. 4(11): p. 2144-2156.

139. Li, Y. and D. Boraschi, Endotoxin contamination: a key element in the interpretation of nanosafety studies. Nanomedicine (Lond), 2016. 11(3): p. 269-87.

140. Samburova, V., B. Zielinska, and A. Khlystov, Do 16 Polycyclic Aromatic Hydrocarbons Represent PAH Air Toxicity? Toxics, 2017. 5(3).

141. Birch, M.E., Exposure and emissions monitoring during carbon nanofiber production--Part II: polycyclic aromatic hydrocarbons. Ann Occup Hyg, 2011. 55(9): p. 1037-47.

142. Pal, A.K., et al., Screening for oxidative damage by engineered nanomaterials: a comparative evaluation of FRAS and DCFH. Journal of Nanoparticle Research, 2014. 16(2).

143. Kisin, E.R., et al., Genotoxicity of carbon nanofibers: are they potentially more or less dangerous than carbon nanotubes or asbestos? Toxicol Appl Pharmacol, 2011. 252(1): p. 1-10.

144. économiques, O.d.c.e.d.d., Test No. 487: In vitro mammalian cell micronucleus test. 2016: OECD Publishing.

145. FDA, International Conference on Harmonisation; guidance on S2 (R1) Genotoxicity Testing and Data Interpretation for Pharmaceuticals intended for Human Use; availability. Notice. 2012. 77(110): p. 33748.

146. Moller, P., et al., Role of oxidative stress in carbon nanotube-generated health effects. Arch Toxicol, 2014. 88(11): p. 1939-64.

147. Shvedova, A.A., et al., Mechanisms of carbon nanotube-induced toxicity: focus on oxidative stress. Toxicol Appl Pharmacol, 2012. 261(2): p. 121-33.

148. Kuempel, E.D., et al., Evaluating the mechanistic evidence and key data gaps in assessing the potential carcinogenicity of carbon nanotubes and nanofibers in humans. Crit Rev Toxicol, 2017. 47(1): p. 1-58.

149. Moller, P. and N.R. Jacobsen, Weight of evidence analysis for assessing the genotoxic potential of carbon nanotubes. Crit Rev Toxicol, 2017. 47(10): p. 867-884.

150. Magdolenova, Z., et al., Mechanisms of genotoxicity. A review of in vitro and in vivo studies with engineered nanoparticles. Nanotoxicology, 2014. 8(3): p. 233-78.

151. Guo, X. and T. Chen, Progress in Genotoxicity Evaluation of Engineered Nanomaterials, in NanomaterialsToxicity and Risk Assessment. 2015.

152. Saleh, D.M., et al., Comparative carcinogenicity study of a thick, straight-type and a thin, tangled-type multi-walled carbon nanotube administered by intra-tracheal instillation in the rat. Particle and Fibre Toxicology, 2020. 17(1): p. 48.

153. Meng, H., et al., A predictive toxicological paradigm for the safety assessment of nanomaterials. ACS Nano, 2009. 3(7): p. 1620-7.

154. Puzyn, T., et al., Using nano-QSAR to predict the cytotoxicity of metal oxide nanoparticles. Nat Nanotechnol, 2011. 6(3): p. 175-8.

155. Yanamala, N., et al., Grouping of carbonaceous nanomaterials based on association of patterns of inflammatory markers in BAL fluid with adverse outcomes in lungs. Nanotoxicology, 2019. 13(8): p. 11021116.

156. Yanamala, N., et al., Sparse Supervised Classification Methods Predict and Characterize Nanomaterial Exposures: Independent Markers of MWCNT Exposures. Toxicol Pathol, 2018. 46(1): p. 14-27.

157. Gandon, A., et al., Surface reactivity measurements as required for grouping and read-across: An advanced FRAS protocol. Journal of Physics: Conference Series, 2017. 838.

158. Hsieh, S.F., et al., Mapping the biological oxidative damage of engineered nanomaterials. Small, 2013. 9(910): p. 1853-65.

159. Rogers, E.J., et al., A high throughput in vitro analytical approach to screen for oxidative stress potential exerted by nanomaterials using a biologically relevant matrix: human blood serum. Toxicol In Vitro, 2008. 22(6): p. 1639-47. 
160. Reddel, R.R., et al., Transformation of Human Bronchial Epithelial Cells by Infection with SV4O or Adenovirus-12 SV40 Hybrid Virus, or Transfection via Strontium Phosphate Coprecipitation with a Plasmid Containing SV4O Early Region Genes. 1988. 48(7): p. 1904-1909.

161. Gwinn, M.R., et al., The Role of p53 in Silica-Induced Cellular and Molecular Responses Associated with Carcinogenesis. Journal of Toxicology and Environmental Health, Part A, 2009. 72(23): p. 1509-1519.

162. Sargent, L.M., S.H. Reynolds, and V. Castranova, Potential pulmonary effects of engineered carbon nanotubes: in vitro genotoxic effects. Nanotoxicology, 2010. 4(4): p. 396-408.

163. Sargent, L.M., et al., Induction of aneuploidy by single-walled carbon nanotubes. 2009. 50(8): p. 708-717.

164. Erdely, A., et al., Carbon nanotube dosimetry: from workplace exposure assessment to inhalation toxicology. Particle and Fibre Toxicology, 2013. 10(1): p. 53.

165. Stone, K.C., et al., Distribution of Lung Cell Numbers and Volumes between Alveolar and Nonalveolar Tissue. 1992. 146(2): p. 454-456.

166. Porter, D., et al., A biocompatible medium for nanoparticle dispersion. Nanotoxicology, 2008. 2(3): p. 144154.

167. Huang, X. and Z. Darzynkiewicz, Cytometric Assessment of Histone H2AX Phosphorylation, in DNA Repair Protocols: Mammalian Systems, D.S. Henderson, Editor. 2006, Humana Press: Totowa, NJ. p. 73-80.

168. Théry, M. and M. Bornens, Get round and stiff for mitosis. HFSP journal, 2008. 2(2): p. 65-71.

169. Breiman, L., Random Forests. Machine Learning, 2001. 45(1): p. 5-32.

170. Kursa, M.B. and W.R. Rudnicki, Feature Selection with the Boruta Package. Journal of Statistical Software, 2010. 36(11).

171. R Core, T. R: A language and environment for statistical computing. 2016; Available from: https://www.Rproject.org/.

172. Aiso, S., et al., Pulmonary toxicity of intratracheally instilled multiwall carbon nanotubes in male Fischer 344 rats. Ind Health, 2010. 48(6): p. 783-95.

173. Morimoto, Y., et al., Pulmonary toxicity of well-dispersed multi-wall carbon nanotubes following inhalation and intratracheal instillation. Nanotoxicology, 2012. 6(6): p. 587-599.

174. Hanahan, D. and R.A. Weinberg, Hallmarks of cancer: the next generation. Cell, 2011. 144(5): p. 646-74.

175. Hanahan, D. and R.A. Weinberg, The hallmarks of cancer. Cell, 2000. 100(1): p. 57-70.

176. Fraser, K., et al., Physicochemical characterization and genotoxicity of the broad class of carbon nanotubes and nanofibers used or produced in U.S. facilities. Particle and Fibre Toxicology, 2020. 17(1): p. 62.

177. Nygaard, U.C., et al., Carbon nanofibers have IgE adjuvant capacity but are less potent than nanotubes in promoting allergic airway responses. Biomed Res Int, 2013. 2013: p. 476010.

178. Zeidler-Erdely, P.C., A. Erdely, and J.M. Antonini, Immunotoxicology of arc welding fume: worker and experimental animal studies. J Immunotoxicol, 2012. 9(4): p. 411-25.

179. Rao, G.V., et al., Efficacy of a technique for exposing the mouse lung to particles aspirated from the pharynx. J Toxicol Environ Health A, 2003. 66(15): p. 1441-52.

180. Kodali, V.K., et al., Acute in vitro and in vivo toxicity of a commercial grade boron nitride nanotube mixture. Nanotoxicology, 2017. 11(8): p. 1040-1058.

181. Xia, T., et al., Interlaboratory evaluation of in vitro cytotoxicity and inflammatory responses to engineered nanomaterials: the NIEHS Nano GO Consortium. Environmental health perspectives, 2013. 121(6): p. 683690.

182. Fraser, K., et al., Physicochemical characterization and genotoxicity of the broad class of carbon nanotubes and nanofibers used or produced in U.S. facilities. Part Fibre Toxicol, 2020. 17(1): p. 62.

183. Choi, H.S., et al., Rapid translocation of nanoparticles from the lung airspaces to the body. Nat Biotechnol, 2010. 28(12): p. 1300-3.

184. Harmsen, A.G., et al., The role of macrophages in particle translocation from lungs to lymph nodes. Science, 1985. 230(4731): p. 1277-80.

185. Weber, E., et al., Pulmonary lymphatic vessel morphology: a review. Ann Anat, 2018. 218: p. 110-117.

186. Mishra, A., et al., Identification of TGF-B receptor-1 as a key regulator of carbon nanotube-induced fibrogenesis. Am J Physiol Lung Cell Mol Physiol, 2015. 309(8): p. L821-33. 
187. Lamouille, S., J. Xu, and R. Derynck, Molecular mechanisms of epithelial-mesenchymal transition. Nature reviews. Molecular cell biology, 2014. 15(3): p. 178-196.

188. Ma, J., et al., Role of epithelial-mesenchymal transition (EMT) and fibroblast function in cerium oxide nanoparticles-induced lung fibrosis. Toxicology and applied pharmacology, 2017. 323: p. 16-25.

189. Moustakas, A. and C.-H. Heldin, Mechanisms of TGFB-Induced Epithelial-Mesenchymal Transition. Journal of clinical medicine, 2016. 5(7): p. 63.

190. Ruenraroengsak, P., et al., Translocation of Functionalized Multi-Walled Carbon Nanotubes across Human Pulmonary Alveolar Epithelium: Dominant Role of Epithelial Type 1 Cells. ACS Nano, 2016. 10(5): p. 507085.

191. Pacurari, M., et al., Cell permeability, migration, and reactive oxygen species induced by multiwalled carbon nanotubes in human microvascular endothelial cells. J Toxicol Environ Health A, 2012. 75(3): p. 129-47.

192. Chen, Y., Q. Yu, and C.-B. Xu, A convenient method for quantifying collagen fibers in atherosclerotic lesions by ImageJ software. Int J Clin Exp Med, 2017. 10: p. 14927-14935.

193. Davidson, D.C., et al., Direct stimulation of human fibroblasts by $n \mathrm{CeO} 2$ in vitro is attenuated with an amorphous silica coating. Particle and fibre toxicology, 2016. 13(1): p. 23-23.

194. Mishra, A., et al., Assessment of Pulmonary Fibrogenic Potential of Multiwalled Carbon Nanotubes in Human Lung Cells. Journal of Nanomaterials, 2012. 2012: p. 930931.

195. Wang, L., et al., Direct fibrogenic effects of dispersed single-walled carbon nanotubes on human lung fibroblasts. J Toxicol Environ Health A, 2010. 73(5): p. 410-22.

196. Hansen, S.F. and A. Lennquist, Carbon nanotubes added to the SIN List as a nanomaterial of Very High Concern. Nature Nanotechnology, 2020. 15(1): p. 3-4.

197. Fadeel, B. and K. Kostarelos, Grouping all carbon nanotubes into a single substance category is scientifically unjustified. Nat Nanotechnol, 2020. 15(3): p. 164. 